\title{
Aspects of heavy, dense lattice QCD: series expansion methods, baryon versus isospin density and large $N_{c}$
}

\author{
Dissertation \\ zur Erlangung des Doktorgrades \\ der Naturwissenschaften
}

Vorgelegt beim Fachbereich Physik

der Johann Wolfgang Goethe-Universität

in Frankfurt am Main

Von

Jonas Benedict Scheunert

aus Ulm, Deutschland

Frankfurt am Main 2021

D30 
vom Fachbereich Physik der

Johann Wolfgang Goethe-Universität als Dissertation angenommmen.

Dekan: Prof. Dr. Appelshäuser

Gutachter: Prof. Dr. O. Philipsen

Prof. Dr. D. H. Rischke

Datum der Disputation: 


\section{Contents}

Abstract $v$

Deutsche Zusammenfassung vii

1 Introduction $\quad 1$

2 Basics $\quad \mathbf{5}$

2.1 Effective Polyakov loop theories of lattice QCD . . . . . . . . . . . . 5

2.1.1 Evaluation of Polyakov loop theories with a sign problem . . . . . . . 5

2.1.2 Derivation of the effective actions . . . . . . . . . . . . 5

2.1.3 Results from the effective theory . . . . . . . . . . . . . . . 13

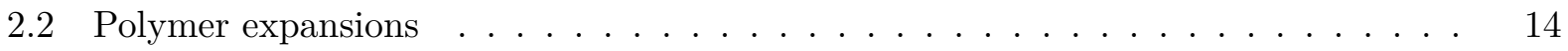

2.2 .1 Sums over disjoint subsets . . . . . . . . . . . . . . . 15

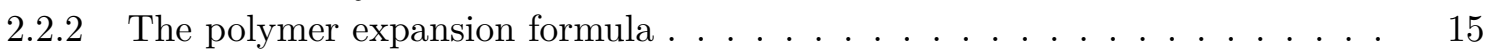

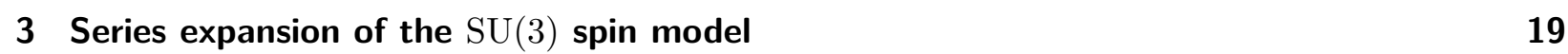

3.1 Weak graph expansion of the spin model . . . . . . . . . . . . . . . . . . 20

3.2 Free graph expansion of the spin model . . . . . . . . . . . . . . . . 24

3.2 .1 Unrenormalized expansion . . . . . . . . . . . . . . . . . . 24

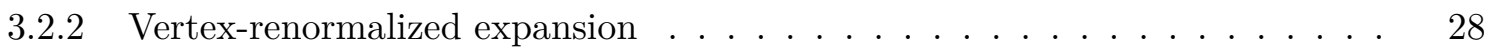

3.2.3 Example: Deriving the free energy density to $\mathcal{O}\left(\tau^{2}\right) \ldots \ldots \ldots$

3.3 Results from the series expansion . . . . . . . . . . . . . . 33

4 The hopping expansion as a polymer expansion, logarithmic resummations 37

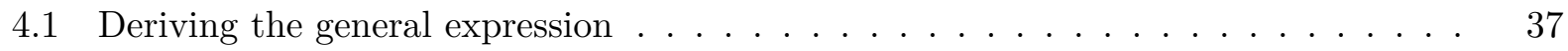

4.2 Equivalent graph weights . . . . . . . . . . . . . . . . . . . 42

4.3 Example: Deriving the $\kappa^{4}$-action $\ldots \ldots \ldots \ldots \ldots \ldots$

4.4 Logarithmic resummations . . . . . . . . . . . . . . . . . . . . . . 48

4.4 .1 Resummation of a single graph weight . . . . . . . . . . . . 48

4.4.2 On the importance of the logarithmic resummation when evaluating the effective theory . . . . . . . . . . . . . . . 49

4.4.3 Logarithmic resummation of all graph combinations . . . . . . . . . . . 50

4.4.4 Resummation of the $\kappa^{4}$-action $\ldots \ldots \ldots \ldots \ldots \ldots$

4.5 Ladder resummation . . . . . . . . . . . . . . . . . . . . . . 52

4.6 Direct evaluation vs evaluation of the effective theory $\ldots \ldots \ldots \ldots$

4.6.1 Direct evaluation of $\log (Z) \ldots \ldots \ldots \ldots \ldots$. . . . . . . . . 57

4.6.2 Analytic evaluation of the effective theory . . . . . . . . . . . . 59

4.6.3 Evaluation of the temporal gauge integrals for the case $N_{c}=3 \ldots \ldots$

4.6 .4 Partial contractions . . . . . . . . . . . . . . . 64

$\begin{array}{lll}5 & \text { The onset transition for baryon and isospin chemical potential } & \mathbf{6 7}\end{array}$

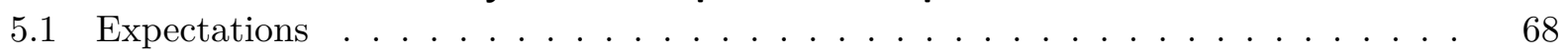

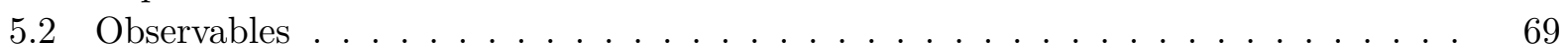

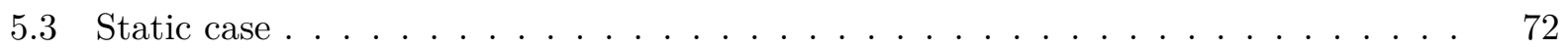




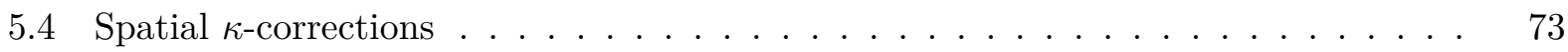

6 Large $N_{c}$ behavior of the effective theory, quarkyonic matter $\quad 77$

6.1 Evaluation of the effective theory for arbitrary $N_{c} \ldots \ldots \ldots \ldots \ldots$

6.1.1 Integration of class functions . . . . . . . . . . . . . . . . . 80

6.1.2 Evaluation of the temporal gauge integrals to $\mathcal{O}\left(\kappa^{4}\right) \ldots \ldots \ldots$

6.2 The onset transition in the large $N_{c}$ limit $\ldots \ldots \ldots \ldots \ldots$

6.3 Thermodynamic observables for large $N_{c} \ldots \ldots \ldots \ldots \ldots$. . . . . 87

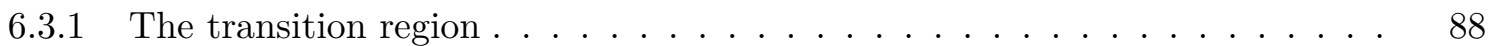

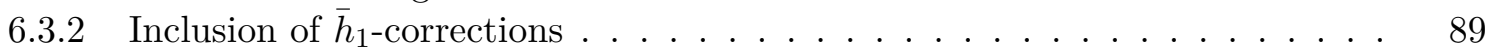

6.3.3 Gauge corrections and 't Hooft scaling . . . . . . . . . . . . . . . . . . 90

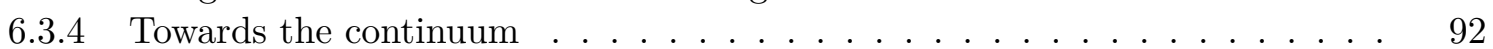

6.3.5 The phase diagram for growing and large $N_{c} \ldots \ldots \ldots \ldots$. . . . . 94

6.3.6 Quarkyonic matter and lattice saturation . . . . . . . . . . . . 95

7 Conclusions and Research perspectives $\quad 97$

$\begin{array}{ll}\text { A Notations } & 101\end{array}$

$\begin{array}{ll}\text { B Graphs } & 103\end{array}$

$\begin{array}{ll}\text { C The } \mathrm{SU}(N) \text { one link integral } & 105\end{array}$

$\begin{array}{ll}\text { D A determinant evaluation } & 109\end{array}$ 


\section{Abstract}

For finite baryon chemical potential, conventional lattice descriptions of quantum chromodynamics (QCD) have a sign problem which prevents straightforward simulations based on importance sampling. In this thesis we investigate heavy dense QCD by representing lattice QCD with Wilson fermions at finite temperature and density in terms of Polyakov loops. We discuss the derivation of 3-dimensional effective Polyakov loop theories from lattice QCD based on a combined strong coupling and hopping parameter expansion, which is valid for heavy quarks. The finite density sign problem is milder in these theories and they are also amenable to analytic evaluations.

The analytic evaluation of Polyakov loop theories via series expansion techniques is illustrated by using them to evaluate the $\mathrm{SU}(3)$ spin model. We compute the free energy density to 14th order in the nearest neighbor coupling and find that predictions for the equation of state agree with simulations to $\mathcal{O}(1 \%)$ in the phase were the (approximate) $Z(3)$ center symmetry is intact. The critical end point is also determined but with less accuracy and our results agree with numerical results to $\mathcal{O}(10 \%)$. While the accuracy for the endpoint is limited for the current length of the series, analytic tools provide valuable insight and are more flexible. Furthermore they can be generalized to Polyakov-loop-theories with $n$-point interactions.

We also take a detailed look at the hopping expansion for the derivation of the effective theory. The exponentiation of the action is discussed by using a polymer expansion and we also explain how to obtain logarithmic resummations for all contributions, which will be achieved by employing the finite cluster method know from condensed matter physics. The finite cluster method can also be used to evaluate the effective theory and comparisons of the evaluation of the effective action and a direction evaluation of the partition function are made. We observe that terms in the evaluation of the effective theory correspond to partial contractions in the application of Wick's theorem for the evaluation of Grassmann-valued integrals. Potential problems arising from this fact are explored.

Next to next to leading order results from the hopping expansion are used to analyze and compare the onset transition both for baryon and isospin chemical potential. Lattice QCD with an isospin chemical potential does not have a sign problem and can serve as a valuable cross-check. Since we are restricted by the relatively short length of our series, we content ourselves with observing some qualitative phenomenological properties arising in the effective theory which are relevant for the onset transition.

Finally, we generalize our results to arbitrary number of colors $N_{c}$. We investigate the transition from a hadron gas to baryon condensation and find that for any finite lattice spacing the transition becomes stronger when $N_{c}$ is increased and to be first order in the limit of infinite $N_{c}$. Beyond the onset, the pressure is shown to scale as $p \sim N_{c}$ through all available orders in the hopping expansion, which is characteristic for a phase termed quarkyonic matter in the literature. Some care has to be taken when approaching the continuum, as we find that the continuum limit has to be taken before the large $N_{c}$ limit. Although we currently are unable to take the limits in this order, our results are stable in the controlled range of lattice spacings when the limits are approached in this order. 



\section{Deutsche Zusammenfassung}

Die Quantenchromodynamik (QCD) ist die etablierte Theorie zur Beschreibung der starken Wechselwirkung und bildet neben der Theorie der elektroschwachen Wechselwirkung (welche in dieser Arbeit vernachlässigt wird) einen Grundpfeiler des Standardmodells der Teilchenphysik. Die laufende Kopplung der QCD hat die Eigenschaft, dass sie der Größenordnung eins ist für Prozesse deren Energieskala kleiner als $\Lambda_{\mathrm{QCD}} \approx 150 \mathrm{MeV}$ ist. Aus diesem Grund können derartige Prozesse nicht mit denen in der Quantenfeldtheorie häufig benutzten Methoden der Störungsentwicklung um den Grenzfall der verschwindenden Kopplung behandelt werden. Ein erfolgreicher Ansatz mit diesem Problem umzugehen, ist die Theorie auf einem diskretem Gitter zu behandeln anstelle der kontinuierlichen Raumzeit. Die daraus resultierende Gitter-QCD kann benutzt werden, nicht-perturbative Phänomene der QCD ab-initio mithilfe von Computersimulationen zu untersuchen. Durch die Diskretisierung wird zwar aus dem Feynman'schen Pfadintegral ein wohldefiniertes gewöhnliches Integral, eine direkte numerische Auswertung dieses Integrals ist allerdings nicht möglich, da die Dimension des Integrals sehr groß ist. Aus diesem Grund werden für die Simulationen stochastische MontCarlo-Simulationen benutzt, bei denen nur die Feldkonfigurationen betrachtet werden, die den wichtigsten Beitrag zum Endergebnis liefern.

Ein wichtiger Aspekt der QCD ist das Phasendiagramm bei endlicher Temperatur und Dichte. Führt man allerdings baryochemisches Potential in die Gitter-QCD ein, tritt ein schwerwiegendes technisches Problem auf. Die Teilchen-Antiteilchen Asymmetrie, die durch das baryochemische Potential verursacht wird, führt dazu, dass die Fermionen-Determinante komplex wird und die Verteilung der Feldkonfigurationen nicht einer positiv definiten Boltzmannverteilung entspricht. Dieses Vorzeichenproblem der QCD bei endlicher Dichte verhindert eine direkte Anwendung von Monte-Carlo-Methoden in Simulationen. Es gibt verschiedene Ansätze dieses Vorzeichenproblem zu umgehen, bisher ist es jedoch nicht gelungen die QCD bei großen Dichten und niedrigen Temperaturen verlässlich zu behandeln. In dieser 


\section{Deutsche Zusammenfassung}

Arbeit untersuchen wir QCD bei endlichen Dichte und niedriger Temperatur mithilfe einer Darstellung der Gitter-QCD mit Wilson-Fermionen durch eine Polyakov-loop-Theorie verringerter Dimension.

In Kapitel 2 führen wir Polyakov-loop-Theorien ein und diskutieren zunächst, warum sie bei der Lösung des Vorzeichenproblems helfen. Die Polyakov-loop-Theorien erben zwar das Vorzeichenproblem der zugrundeliegenden Gitter-QCD, es gibt jedoch mehrere Ansätze Polyakov-loop-Theorien dennoch erfolgreich auszuwerten. Dazu gehören die direkte Simulation mithilfe von Reweighting oder mittels der Complex-Langevin-Methode, Dualisierung durch Überführung in eine "flux"-Darstellung, analytische Auswertung durch Reihenentwicklung und die Molekularfeldmethode, welche für Polyakov-loop-Theorien mit weitreichenden Wechselwirkungen sehr gute Resultate liefert. Überzeugt davon, dass Polyakov-loop-Theorien bei der Lösung des Vorzeichenproblems helfen, erklären wir, wie diese aus der Gitter-QCD gewonnen werden können. Im Prinzip gibt es zugehörig zu einer Gitterdiskretisierung der QCD bei endlicher Temperatur genau eine Polyakov-loop-Theorie. Sie ist die Theorie, die man erhält, wenn alle Felder bis auf die zeitlichen Eichtransporter aus der Zustandssumme integriert werden. Dies ist nicht ohne Weiteres möglich und man muss Approximationen einführen oder gewisse Ansätze für die resultierende Theorie machen. Aus diesem Grund sprechen wir oft von Theorien. Zur Herleitung wird in dieser Dissertation eine kombinierte Starkkopplungsentwicklung und Hopping-Parameter-Entwicklung um schwere Quarkmassen benutzt. Dieser Ansatz erlaubt die analytische Auswertung der notwendigen Integrale. Die Entwicklungen wurden in vorherigen Arbeiten benutzt und wir fassen die notwendigen mathematischen Grundlagen zu den Entwicklungen und der Auswertung der Integral zusammen. Außerdem diskutieren wir Resultate für das Phasendiagramm schwerer Quarks, die bisher anhand der auf diese Art gewonnenen Polyakov-loop-Theorien erhalten wurden. Zuletzt werden dann in Kapitel 2 noch sogenannte Polymer-Entwicklungen eingeführt. In ihrer abstrakten Formulierung stellen diese einen Zusammenhang zwischen einer großen Klasse von Zustandssummen und deren Logarithmus her. Da die effektive Theorie als Logarithmus der QCD-Zustandssumme abzüglich der Integration zeitlicher Eichtransporter definiert ist, sind Polymer-Entwicklungen ein wichtiges Werkzeug für die Herleitung der Theorie. Wir werden insbesondere genau erklären wie die Entwicklung des Logarithmus zu einer Entwicklung führt, die im Sinne von zusam- 
menhängender Graphen dargestellt werden kann.

Polymer-Entwicklungen können auch für die analytische Auswertung effektiver Polyakovloop-Theorien durch Reihenentwicklung benutzt werden. Dies wird in Kapitel 3 anhand des sogenannten SU(3)-Spin-Modells untersucht. Das SU(3)-Spin-Modell ist die Polyakov-loopTheorie, die man erhält wenn man für beide oben erwähnten Entwicklungen, die zur Herleitung der effektiven Theorie benutzt werden, jeweils nur die Terme führender Ordnung benutzt. Somit stellt sie eine krude Approximation der Gitter-QCD dar, enthält aber bereits dennoch wichtige Eigenschaften der Wirkung der QCD wie die $Z(3)$-Zentrumssymmetrie bei unendlicher Quarkmasse und die Roberge-Weiss-Symmetrie für komplexes chemisches Potential. Da das Modell auch ein Vorzeichenproblem bei endlicher Dichte hat und bereits erfolgreich mit verschiedenen Methoden ausgewertet wurde, stellt es ein optimalen Testfall für die Anwendung neuer Strategien zur Lösung von Polyakov-loop-Theorien dar. Dazu stellen wir zunächst zwei mögliche Arten der Reihenentwicklung des Spin-Modells vor, die sogenannte Weak-Graph-Entwicklung und die Free-Graph-Entwicklung. Bei beiden Entwicklungen leitet man die Dichte der freie Energie im thermodynamischen Limes als Entwicklung in der Nächsten-Nachbar-Kopplung her und stellt die Koeffizienten der Entwicklung als zusammenhängende Graphen dar. In beiden Fällen muss man abzählen auf wie viele verschiedene Arten die Graphen auf das der Theorie zugrundeliegende Gitter eingebettet werden können, um gewisse kombinatorische Vorfaktoren der Koeffizienten korrekt zu bestimmen. Dabei hat man bei der Free-Graph-Entwicklung den Vorteil, dass bei der Einbettung nur darauf zu achten ist, dass benachbarte Vetices eines Graphs auf nächste Nachbarn des Gitters gesetzt werden. Bei der Weak-Graph-Entwicklung unterliegt man zusätzlich der Einschränkung, dass verschiedene Vertices eines Graphen nicht auf den selben Gitterpunkt gesetzt werden dürfen. Da die Free-Graph-Entwicklung im Fall des Spin-Modells somit einfacher durchzuführen ist untersuchen wir diese genauer und die Weak-Graph-Entwicklung sollte lediglich als Einführung in die kompliziertere Entwicklung in Kapitel 4 betrachtet werden. Ein weiterer Vorteil der Free-Graph-Entwicklung ist, dass durch die geringen Einschränkungen an die Einbettungen sogenannte Renormierungsprozeduren durchgeführt werden können, welche allerdings nicht mit denen aus der Quantenfeldtheorie bekannten Renormierungen zur Entfernung von Ultraviolett-Divergenzen zu verwechseln sind. Wir zeigen wie eine sogenannte 


\section{Deutsche Zusammenfassung}

Vertex-Renormierung beim Spin-Modell durchgeführt werden kann, mithilfe derer man die graphentheoretische Komplexität der Entwicklung verringer kann. Dies führt dazu, dass man bei der Entwicklung nur solche zusammenhängende Graphen betrachten muss, die nicht auseinander fallen, wenn ein Vertex und alle Kanten, die diesen Vertex berühren, entfernt werden. Mithilfe dieser Renormierung ermitteln wir die Dichte der freie Energie zur 14ten Ordnung in der Nächsten-Nachbar-Kopplung. Mit verschiedenen Methoden untersuchen wir die ermittelte Reihe dann und die resultierende Zustandsgleichung stimmt in der Phase, in der die Zentrumssymmetrie nicht spontan gebrochen ist, zu ungefähr 1\% mit dem Endergebnis numerischer Simulationen überein. Die Bestimmung des Phasenübergangs ist schwieriger und für Fälle in denen ein Übergang erste Ordnung vorliegt kann nur eine obere Grenze gegeben werden. Das Spin-Modell hat einen Phasenübergang erster Ordnung der in einem kritischen Punkt zweiter Ordnung endet. Dieser Endpunkt kann gut mithilfe von Padé-Approximanten beschrieben werden und durch die Ermittlung von Schnittpunkten verschiedener Padé-Approximanten verschiedener Observablen gelingt die Bestimmung des Endpunkts mit einer Genauigkeit der Größenordnung $\mathcal{O}(10 \%)$. Die analytische Vorgehensweise kann somit nicht immer mit der Genauigkeit numerischer Simulationen mithalten, ist jedoch flexibler und kann auch auf Polyakov-loop-Theorien mit $n$-Punktkopplungen angewendet werden.

In Kapitel 4 untersuchen wir die Hopping-Parameter-Entwicklung zur Herleitung der effektiven Theorie genauer. Im Gegenteil zu vorherigen Arbeiten wählen wir einen Ansatz, bei denen die räumlichen Eichfelder vor den Graßmann-wertigen Fermionenfeldern ausintegriert werden. Wir erklären wie die in anderen Arbeiten vernachlässigten $N_{\tau}$-Korrekturen systematisch berechnet werden können und wie die effective Wirkung als Exponent geschrieben werden kann mithilfe einer Polymer-Entwicklung. Der graphische Methode die sich daraus zur Herleitung der effektiven Theorie ergibt erleichtert die Diskussion von logarithmischen Resummationen. Wir argumentieren, dass diese logarithmischen Resummationen wichtig sind um Verletzungen der Einschränkungen, die durch die Nilpotenz der Graßmannzahlen gegeben sind, zu vermeiden. Ein systematische Herleitung aller logarithmischer Resummationen kann auf natürliche Weise mithilfe der Finite-Cluster-Methode erzielt werden, welche aus der Festkörperphysik bekannt ist. Als Abschluss des Kapitels zeigen wir wie die effektive Theorie mithilfe der Finite-Cluster-Methode auch ausgewertet werden kann und vergleichen 
die direkte Auswertung der Zustandssumme der QCD mit der Auswertung der effektiven Theorie. Hierbei stellt sich heraus, dass die effektive Theorie zu Termen führen kann, die einer teilweisen Anwendungen des Wick'schen Theorems zur Auswertung von GraßmannIntegralen entsprechen. Mögliche Probleme die sich anhand dieser Tatsache ergeben werden diskutiert. Als Nebenprodukt dieses Kapitels wir die effektive Theorie bis zur vierten Ordnung im Hopping-Parameter hergeleitet und ausgewertet. Diese Entwicklung wird dann in den darauf folgenden Kapiteln ausgewertet.

In Kapitel 5 wird zunächst die Onset-Transition, die bei niedriger Temperatur auftritt, untersucht. Hierbei werden sowohl der Fall mit baryochemischem Potential als auch der Fall mit Isospin-chemischem Potential untersucht. Neutronensterne sind typische Systeme bei denen eine Isospin-Asymmetrie vorliegt. Wir beschränken uns allerdings auf die Untersuchung von Systemen in denen entweder nur baryochemisches Potential oder nur Isospin-chemisches Potential vorliegen. Letztere treten zwar in der Natur nicht auf, sind aber theoretisch gut verstanden, da sie kein Vorzeichenproblem haben und liefern somit gute Möglichkeit die Ergebnisse der effektiven Theorie zu überprüfen. In der Tat kann der Onset bei verschwindender Temperatur in beiden Fällen anhand relativ einfacher phänomenologischer Tatsachen und statistischer Argumente verstanden werden. Da wir mit der relativ kurzen Reihenentwicklung die uns zu diesem Zeitpunkt zur Verfügung steht keine Verlässliche Aussagen zu Phasenübergängen machen können, begnügen wir uns damit zu überprüfen ob wir phänomenologischen Tatsachen, die für die charakteristischen Eigenschaften des Onsets verantwortlich sind, qualitativ reproduzieren können. Wir finden Hinweise darauf, dass die effektive Theorie korrekt das Auftreten von attraktiven Nukleonen beziehungsweise repulsiven Pionen als effektive Freiheitsgrade der QCD vorhersagt.

Anschließend verallgemeinern wir in Kapitel 6 unsere Resultate auf eine beliebige Anzahl an Farben $N_{c}$. Wir sehen, dass in diesem Fall der Onset von der Hadronen-Gas-Phase zur kondensierten nuklearen Materie für alle untersuchten Gitterabstände stärker wird wenn $N_{c}$ erhöht wird und im Limes $N_{c} \rightarrow \infty$ stets erster Ordnung ist. Darüber hinaus stellen wir fest, dass in der Phase mit kondensierten Baryonen der Druck gemäß $p \sim N_{c}$ skaliert in allen Ordnungen der Hopping-Parameter-Entwicklung die uns zur Verfügung stehen. Dieses Verhalten des Drucks führt zur Vorhersage dass es sich hierbei um eine sogenannte quarkyonische Phase 
handelt, die sowohl baryonische als auch Quark-artige Eigenschaften hat. Sie unterschiedet sich von der Hadronen-Gas-Phase, deren Druck mit $N_{c}^{0}$ skaliert, und dem Quark-GluonenPlasma, bei dem $p \sim N_{c}^{2}$. Da den Ergebnissen eine diskreten Raumzeit unterliegt sind diese durch die Ausbildung eines Kristalls saturierter Quarkmaterie aufgrund des Pauli-Prinzips verfälscht. Zwar ist das Bild der quarkyonischen Materie verträglich mit der Ausbildung eines solchen Kristalls, für Kontinuumsphysik sollte allerdings der Kontinuumslimes betrachtet werden und wir sehen dass dieser vor dem Limes $N_{c} \rightarrow \infty$ genommen werden muss. Dies ist anhand der Reihenentwicklung die uns im Moment zur Verfügung steht nicht möglich, im Bereich der Gitterabstände die unter Kontrolle sind scheinen unsere Ergebnisse allerdings mit der korrekten Reihenfolge der Grenzwerte verträglich.

Abschließend geben wir in Kapitel 7 eine Fazit unserer Ergebnisse und einen Ausblick auf mögliche Projekte, die auf den Resultaten der Dissertation aufbauen und diese erweitern könnten. Hierbei sei insbesondere auf die Möglichkeit hingewiesen, die Finite-Cluster-Methode in einer numerischen Bestimmung der effektiven Kopplungen der Polyakov-loop-Theorie zu benutzen.

In einem Appendix werden unter anderem im Haupttext benötigte graphentheoretische Begriffe zusammengetragen und ein erzeugendes Funktional für $\mathrm{SU}(N)$-Integrale hergeleitet. Außerdem werden einige Notationen die im Text eingeführt wurden aufgeführt. 


\section{Introduction}

With the discovery of the Higgs boson [1,2], the last missing piece in the description of the Standard Model of particle physics has been found. Although it is often said in popular scientific articles that the Higgs particle is the particle "that gives you mass", the main contribution to the mass of everyday objects is actually dynamically generated according to the Standard Model. This dynamical mass generation is due to the strong interactions, of which quantum chromodynamics (QCD) is the established theory. Besides the electroweak interactions (which we will neglect in this thesis), QCD is one pillar of the Standard Model. It describes not only the main contribution of the interactions between nucleons and other hadrons, but also the structure and the dynamically generated masses of these particles, which are built out of the fundamental degrees of freedoms of QCD, quarks and gluons. In the language of quantum field theory, QCD is an SU(3) Yang-Mills gauge theory and the gauge fields are coupled to $N_{f}=6$ fermion fields. The elementary excitations of the gauge fields correspond to the massless spin-1 gluons, while the fermion fields correspond to the massive quarks with spin $1 / 2$. Despite describing a large range of physical phenomenon, the six masses of the quarks and the strong-interaction coupling constant $g$ are the only free parameters of the theory.

The renormalization procedures necessary to do calculations in the theory introduce a dependence of the coupling on the energy scale of the process that one is describing, a phenomenon called running coupling. For asymptotically large energies the coupling goes to zero (asymptotic freedom), while at the characteristic energy scale of QCD $\Lambda_{\mathrm{QCD}} \approx 150 \mathrm{MeV}$ it becomes of order one (infrared slavery), necessitating a non-perturbative description. This infrared slavery is often said to be the reason for the observation that the bound hadrons which are the asymptotic particle states of QCD are singlets under transformations of the SU(3) "color" group, the phenomenon of color confinement. However, the asymptotic particles of a gauge-Higgs theory are also color singlets and the dynamics of such a theory is similar to that of the weak interactions. Indeed, the question on how to best define confinement such that it characterizes the situation in QCD best is actually not completely clear and for an overview of the subtleties involved we refer to [3].

Independent of the subtleties surrounding confinement, perturbative methods based on the expansion in the strong coupling constant $g$ are certainly not valid at low energies and one needs alternative methods that can describe non-perturbative phenomenon like dynamical mass generation. One such approach, enabling ab-initio calculation of non-perturbative phenomena in QCD, is the lattice formulation of QCD. Originally proposed by Kenneth Wilson [4], it replaces the continuous space-time by a discrete lattice with a minimal length scale. In this way a cut-off is implemented and the Feynman path integrals of the theory are well-defined. This discretization is implemented in a way such that important properties of the continuum theory are preserved, namely gauge invariance and the positivity of the metric of the Hilbert space of physical states [5]. For fermions, some care has to be taken such that chiral symmetry can be recovered in the continuum without introducing spurious fermion flavors [6-8]. With the help of Monte-Carlo importance sampling and sufficient computing power one can obtain numerical values for observables in the non-perturbative regime which can be related to physical units via scale setting $[9,10]$ and, given small enough lattices, be extrapolated to give results for the continuum theory. Building on improvements of the formulation, algorithmic developments and Moore's law, impressive results concerning the physics of QCD have been achieved, for example the determination of light hadron masses [11] in agreement with results from experiments. 


\section{Introduction}

Despite the success of lattice QCD, there are still open problems. One important question is that of the phase structure of QCD in the $\left(T, \mu_{B}\right)$-plane of finite temperature and finite baryon chemical potential. This phase diagram is not only of theoretical interest for the general understanding of QCD, but also plays an important role in cosmology and astrophysics, as it is relevant for the state of the entire universe shortly after the Big Bang and also for neutron stars and their mergers in today's universe. Furthermore, there are several experiments that attempt to recreate similar environments by colliding heavy ions in order to probe the thermodynamic properties of strong interacting matter. The Large Hadron Collider (LHC) at Cern in Genf explores the phase diagram at low densities and high temperatures, while the Relativistic Heavy Ion Collider at the Brookhaven national lab in New York can access somewhat larger densities. There are several upcoming facilities that will give data at lower temperatures and even higher baryon densities. These include the Nuclotron-based Ion Collider fAcility (NICA) at the Joint Institute for Nuclear Research (JINR) in Dubna, the Compressed Baryonic Matter Experiment (CBM) at the Facility for Antiproton and Ion Research in Europe (FAIR) at Darmstadt and the J-PARC heavy ion project (J-PARC-HI) at the Japan Proton Accelerator Research Complex (J-PARC) in Tōkai. An understanding of the phase diagram is important to predict or correctly interpret the results of these experiments.

From the theory side, the case $\mu_{B}=0$ is well understood from conventional lattice QCD with importance sampling. For low temperatures the quarks and gluons are confined into hadrons and the chiral symmetry of QCD is spontaneously broken by a non-vanishing quark condensate. Increasing the temperature, there is a chiral and deconfinement crossover transition [12] at a pseudocritical temperature of $T_{p c} \approx 155 \mathrm{MeV}[13,14]$ to a phase commonly termed the quark-gluon plasma (QGP). Recent studies suggest that for temperatures right above the crossover the relevant degrees of freedom are actually chirally symmetric quarks bound by strings of the chromoelectric field $[15,16]$ and it is suggested to term this regime a "stringy fluid" instead of a plasma. Only for temperatures $\gtrsim 1 \mathrm{GeV}$ quasi-free behavior of the quarks and a full restoration of chiral symmetry are observed.

Using lattice QCD, one can also investigate the situation with unphysical quark masses and one observes that depending on the quark masses the crossover can turn into a true phase transition of 1st or 2nd order. Investigating this question for different combinations of the up-, down- and strange-quark masses leads to the so-called Columbia plot, for an overview of recent results see [17]. While this may seem like an academic exercise, it can nevertheless give interesting insights in the structure of QCD and can give ideas for the phase structure for regimes which cannot be reached currently.

For $\mu_{B}>0$, a severe technical problem occurs when one attempts simulations based on lattice QCD. One observes that the particle-antiparticle asymmetry due to the baryon chemical potential destroys the reality of the fermion determinant. Consequently, it is not possible to obtain a real and positive definite Boltzmann weight that is necessary for the application of Monte-Carlo importance sampling. This is known as the finite density sign problem. Several methods have been developed in an attempt to deal with this sign problem $[18,19]$. Of those, reweighting techniques [20-24], analytic continuation from imaginary chemical potential (where no sign problem occurs) [25-28] and the Taylor expansion method [29-34] are limited to rather small chemical potentials or high temperatures $\mu_{B} / T \lesssim 3$. Attempts to study larger chemical potentials and lower temperatures have been made using the complex Langevin approach [35-37], the Lefschetz thimble method [38-42], the density of states method [43-45], a canonical approach [46-48] and by finding dual representations of QCD [49-55]. All these methods have advantages and shortcomings and so far none of them can be used to study QCD at high densities and low temperatures for physical parameters close to the continuum.

In the parameter region $\mu_{B} / T \lesssim 3$, where reliable predictions can be made, there are so far no 
signs that the chiral transition turns critical $[17,56]$. Recently, predictions of a critical point have been made in the range $\mu_{B} / T \approx 4-6$ using Dyson-Schwinger equations [57], the functional renormalization group [58] and a combination of these methods [59], but the systematic uncertainties are not yet under control.

In this thesis we are especially interested in the cold and dense region $\mu_{B} / T \gg 1$. It is expected that there is a nuclear onset transition when going along the chemical potential axis for low temperatures. Beyond that onset there are interesting conjectures about exotic phases, for example quarkyonic matter (which we will discuss in chapter 6) and color superconducting phases [60]. In this thesis, we study cold and dense QCD by considering a dimensional reduced representation of QCD in terms of Polyakov loops. More precisely, we will study 3-dimensional effective Polyakov loop theories of $(3+1)$-dimensional lattice QCD at finite temperature and density.

In chapter 2 we will introduce these theories and discuss some basic aspects concerning their derivation and evaluation. In that chapter, we will also discuss Polymer expansions, as they are a useful tool for deriving and evaluating these theories. After that, in chapter 3, we take a closer look at the analytic evaluation of these theories via series expansion methods by means of a simple model. We will discuss the combinatorial and graph theoretic problems related to the expansion and give a short overview over the results obtained from the series expansion. Chapter 4 is then concerned with an alternative approach to the hopping parameter expansion. The hopping parameter expansion is an expansion around the heavy quark limit that we use for the derivation of the effective theory. Using this approach, we will take a closer look at some of the structural aspects of the hopping expansion. As a side effect, we will derive and evaluate the effective theory to 4th order in the hopping parameter. Based on these results, we will discuss the onset transition both for baryon and isospin chemical potential in chapter 5. Isospin chemical potential is relevant for physical systems like neutron stars. Furthermore, systems with pure isospin chemical potential have no sign problem and are therefore relatively well understood theoretically. Therefore they can serve as a valuable cross-check. Being constrained to relatively low orders in our expansion, we will be mainly concerned with the discussion of qualitative properties. In chapter 6 , we will discuss the generalization of our results to arbitrary number of colors $N_{c}$. Based on this, we will investigate the scaling properties of heavy dense QCD in the limit $N_{c} \rightarrow \infty$ and check if our results are consistent with the conjectures that lead to the prediction of quarkyonic matter. Finally, we will give our conclusions and discuss possible avenues for future research in chapter 7 . We note that in the appendix we collect some notations which are introduced in the main text but might be missed if only certain sections of the text are of interest. 



\section{Basics}

\subsection{Effective Polyakov loop theories of lattice QCD}

As mentioned in the introduction, our tool to deal with the sign problem in lattice QCD are 3dimensional Polyakov loop effective theories of $(3+1)$-dimensional lattice QCD. Since the underlying lattice gauge theory has a sign problem, it is not surprising, that this is also the case for the resulting Polyakov loop action. In what sense then do these theories help when dealing with the sign problem?

\subsubsection{Evaluation of Polyakov loop theories with a sign problem}

Although the effective theories also have a sign problem at finite baryon chemical potential, there have been several successful efforts to deal with this sign problem:

- Reweighting has been applied with fully controlled average sign up to $\mu / T \lesssim 3$ to a nearest neighbor Polyakov loop action [61].

- Furthermore, stochastic quantization using complex Langevin was employed [62-64], also beyond nearest neighbor interactions $[65,66]$.

- Some nearest neighbor actions have also been shown to be solvable by converting the action to a dual "flux" representation [61, 67-70].

- Beyond that the theories are amenable to analytic methods. The mean field approach can be used [63, 71, 72]. A comparison of mean field and complex Langevin results in effective theories with long range interactions have shown nearly perfect agreement between the methods and when they fail to do so, it was found to be due to the branch cut crossing problem in the complex Langevin evolution [66].

- Finally, the analytic evaluation can also be done by series expansion methods leading to a linked cluster expansion. This will be discussed in chapter 3 for a nearest neighbor action to high orders and has been extended to $n$-point interactions in $[73,74]$.

Therefore it seems, that the sign problem is more tractable for Polyakov loop effective theories. The Polyakov loop being the order parameter for the spontaneous breaking of the $Z\left(N_{c}\right)$ center symmetry of pure gauge theory, these theories are not only of practical relevance for the sign problem, but also of theoretical interest. For example, they are the basis of the Svetitsky-Yaffe conjecture concerning the critical behavior of the finite temperature deconfinement transition in gauge theories [75].

Naturally, the next question is how to obtain sensible Polyakov loop actions.

\subsubsection{Derivation of the effective actions}

Dimensionally reduced effective Polyakov loop theories can be derived from full lattice QCD by integrating out all fields except of the temporal gauge transporters. This in itself is of course a challenging task similar to solving full QCD, so some approximations and truncations have to be employed. Therefore, although there is in principle the unique and exact Polyakov loop theory of 
an underlying lattice theory, in practice it makes sense to speak of theories. The earliest work on deriving effective Polyakov loop theories from thermal pure gauge theory based on strong coupling expansion can be found in [76]. Later on, fermions were included by a leading order (heavy quark) hopping expansion $[71,77,78]$, resulting in a nearest neighbor action of the form (the notation will become clear in the next sections)

$$
S_{\mathrm{eff}}=-\sum_{\mathbf{n} \in \Lambda_{s}}\left(\tau \sum_{k=1}^{3}\left[L(\mathbf{n}) L^{*}\left(\mathbf{n}+\mathbf{e}_{k}\right)+L^{*}(\mathbf{n}) L\left(\mathbf{n}+\mathbf{e}_{k}\right)\right]+\kappa e^{\mu} L(\mathbf{n})+\kappa e^{-\mu} L^{*}(\mathbf{n})\right),
$$

where $\mu$ is the chemical potential and $\kappa$ and $\tau$ are effective couplings depending on gauge coupling, quark mass and temperature.

Of course it is important to understand what happens when one moves away from the strong coupling and heavy quark limit. To this end, the authors of [79] considered higher order corrections in a resummed strong coupling expansion, observing much better results than in direct strong coupling calculations of thermodynamic observables [80, 81]. In the same vein, higher order corrections to the hopping expansion were included in $[61,65,73]$. Alternatively, non-perturbative approaches to extract the effective action have been investigated. Early studies employed renormalizationgroup inspired bond moving procedures [82] and microcanonical methods [83]. More recent efforts have been the inverse Monte Carlo method [84, 85], the relative weights method [86-91] and the expectation value matching procedure in [92].

In this thesis, we will focus on the strong coupling and hopping expansion. Note that the nonperturbative approaches usually need some ansatz for the effective action. Since the strong coupling and hopping expansions give general insights about the structure of the Polyakov loop theories, they are also relevant for the non-perturbative determinations.

After defining the Polyakov loop effective theory, we will briefly introduce how the expansions are used to perform the necessary integrations along the lines of $[65,79]$. In anticipation of chapter 6 , we will formulate everything in terms of arbitrary number of colors $N_{c}$ as we did in [93], and therefore will also include results from [94], where the derivation of the effective theory using the expansions has also been discussed for general $N_{c}$.

Our starting point is lattice QCD with standard Wilson fermions and the usual Wilson gauge action.

\section{Lattice QCD at finite temperature and density with Wilson fermions}

Finite temperature is implemented by a compact euclidean time dimension with $N_{\tau}=1 / a T$ slices and (anti-) periodic boundary conditions for (fermions) bosons. Finite chemical potential $\mu$ is introduced by a factor of $\exp ((-) a \mu)$ in front of temporal (anti-) quark hops.

Let us be a bit more specific and also introduce some notation (which largely corresponds to that in [95]). The fundamental quantity we are interested in is the grand-canonical partition function

$$
Z=\int \mathrm{d}[U] \mathrm{d}[\Psi, \bar{\Psi}] e^{-S_{g}[U]-S_{f}[U, \Psi, \bar{\Psi}]}
$$

defined on a 4-dimensional lattice

$$
\Lambda=\left\{n=\left(\tau, n_{1}, n_{2}, n_{3}\right) \mid 0 \leq \tau<N_{\tau}, 0 \leq n_{i}<\sqrt[3]{N_{s}}\right\} .
$$

The partition function contains a path integral over $\mathrm{SU}\left(N_{c}\right)$-valued gauge transporters, which can 
be written as a product of $\mathrm{SU}\left(N_{c}\right)$ Haar integrals

$$
\mathrm{d}[U]=\prod_{n \in \Lambda} \prod_{\mu=0}^{3} \mathrm{~d} U_{\mu}(n) .
$$

The path integral over the Grassmann-valued quark fields reads

$$
\mathrm{d}[\Psi, \bar{\Psi}]=\prod_{n \in \Lambda} \prod_{f, \alpha, c} \mathrm{~d} \Psi(n)_{\alpha, c}^{f} \mathrm{~d} \bar{\Psi}(n)_{\alpha, c}^{f},
$$

where $f, \alpha$ and $c$ refer to flavor-, Dirac- and color indices, respectively.

The Wilson gauge action is defined to be

$$
S_{g}[U]=-\frac{\beta}{2 N_{c}} \sum_{n \in \Lambda} \sum_{\mu<\nu}\left[\operatorname{tr}\left(U_{\mu \nu}(n)\right)+\operatorname{tr}\left(U_{\mu \nu}(n)^{\dagger}\right)\right],
$$

with the so-called plaquette variable $U_{\mu \nu}$

$$
U_{\mu \nu}(n)=U_{\mu}(n) U_{\nu}\left(n+e_{\mu}\right) U_{-\mu}\left(n+e_{\mu}+e_{\nu}\right) U_{-\nu}\left(n+e_{\nu}\right)
$$

and $\beta=2 N_{c} / g^{2}$ denotes the lattice gauge coupling. Writing $P_{\Lambda}$ for the set of all positively oriented plaquettes of the lattice this action can be written in the form

$$
S_{g}[U]=\sum_{p \in P_{\Lambda}} S_{g, P}\left(U_{p}\right)
$$

with

$$
S_{g, P}(U)=-\frac{\beta}{2 N_{c}}\left[\operatorname{tr}(U)+\operatorname{tr}\left(U^{\dagger}\right)\right]
$$

The fields occurring in the gauge action are periodic in all directions of the lattice

$$
\begin{aligned}
& U_{\mu}(n)=U_{\mu}\left(n+N_{\tau} e_{0}\right), \\
& U_{\mu}(n)=U_{\mu}\left(n+\sqrt[3]{N_{s}} e_{i}\right), \forall i \in\{1,2,3\} .
\end{aligned}
$$

The fermion part of the action reads

$$
\begin{aligned}
S_{f}[U, \Psi, \bar{\Psi}]= & \sum_{n \in \Lambda}\{\bar{\Psi}(n) \Psi(n) \\
& -\left[\bar{\Psi}(n) K e^{a \underline{\mu}}\left(1-\gamma_{0}\right) U_{0}(n) \Psi\left(n+e_{0}\right)\right. \\
& \left.+\bar{\Psi}\left(n+e_{0}\right) K e^{-a \underline{\mu}}\left(1+\gamma_{0}\right) U_{0}(n)^{\dagger} \Psi(n)\right] \\
& -\sum_{i=1}^{3}\left[\bar{\Psi}(n) K\left(1-\gamma_{i}\right) U_{i}(n) \Psi\left(n+e_{i}\right)\right. \\
& \left.\left.+\bar{\Psi}\left(n+e_{i}\right) K\left(1+\gamma_{i}\right) U_{i}(n)^{\dagger} \Psi(n)\right]\right\} .
\end{aligned}
$$

Here, we used matrix-vector notation in flavor, Dirac and color space. The hopping parameter matrix $K$ is related to the bare quark masses of the different flavors via

$$
K=\operatorname{diag}\left(\kappa^{(1)}, \ldots, \kappa^{\left(N_{f}\right)}\right)
$$




$$
=\operatorname{diag}\left(1 / 2 a m_{b}^{(1)}+8, \ldots, 1 / 2 a m_{b}^{\left(N_{f}\right)}+8\right)
$$

and $\underline{\mu}$ is simply the diagonal matrix containing the chemical potentials of the different flavors

$$
\underline{\mu}=\operatorname{diag}\left(\mu^{(1)}, \ldots, \mu^{\left(N_{f}\right)}\right) .
$$

Finally the fermions fields are periodic in spatial directions and anti-periodic in temporal direction:

$$
\begin{aligned}
& \Psi(n)=-\Psi\left(n+N_{\tau} e_{0}\right) \\
& \Psi(n)=\Psi\left(n+\sqrt[3]{N_{s}} e_{i}\right), \forall i \in\{1,2,3\}
\end{aligned}
$$

\section{Definition of the effective theory}

As mentioned above, the effective theory is obtained from full lattice QCD by integrating out the Grassmann fields and the spatial gauge links:

$$
\begin{aligned}
Z & =\int \mathrm{d}[U] \mathrm{d}[\Psi, \bar{\Psi}] e^{-S_{g}[U]-S_{f}[U, \Psi, \bar{\Psi}]} \\
& =: \int \mathrm{d}\left[U_{0}\right] e^{-S_{\text {eff }}\left[U_{0}\right]}, \\
\Longrightarrow S_{\text {eff }}\left[U_{0}\right] & =-\log \left(\int \mathrm{d}\left[U_{s}\right] \mathrm{d}[\Psi, \bar{\Psi}] e^{-S_{g}[U]-S_{f}[U, \Psi, \bar{\Psi}]}\right) .
\end{aligned}
$$

Here, $\mathrm{d}\left[U_{0}\right]$ and $\mathrm{d}\left[U_{s}\right]$ denote the integration over the temporal/spatial links

$$
\begin{aligned}
& \mathrm{d}\left[U_{0}\right]=\prod_{n \in \Lambda} \mathrm{d} U_{0}(n), \\
& \mathrm{d}\left[U_{s}\right]=\prod_{n \in \Lambda} \prod_{\mu=1}^{3} \mathrm{~d} U_{\mu}(n) .
\end{aligned}
$$

The measure in equation (2.21) for the integration over the temporal links in equation (2.19) still contains a sum over all lattice points, so why is the effective theory 3-dimensional? It is especially easy to see this by exploiting gauge invariance. To this end, we pick a gauge where all temporal links are equal to $\mathbb{1}$ except of those with $\tau=N_{\tau}-1{ }^{1}$ This means that the link $U_{0}\left(N_{\tau}-1, \mathbf{n}\right)$ will correspond to the whole Wilson line closing through the periodic boundary

$$
W(\mathbf{n})=\prod_{\tau=0}^{N_{\tau}-1} U_{0}(\tau, \mathbf{n}),
$$

and the effective theory only depends on these Wilson lines.

In order to show that it is possible to choose such a gauge, we explicitly give the corresponding gauge transformation following the maximal tree procedure outlined in, for example, [95]. To this end, we write the lattice as a product of spatial lattices obtained from equal time slices $\Lambda=\Lambda_{\tau} \times \Lambda_{s}$, which means,

$$
\begin{aligned}
& \Lambda_{\tau}=\left\{\tau \mid 0 \leq \tau \leq N_{\tau}\right\} \\
& \Lambda_{s}=\left\{\mathbf{n}=\left(n_{1}, n_{2}, n_{3}\right) \mid 0 \leq n_{i} \leq \sqrt[3]{N_{s}}\right\}
\end{aligned}
$$

\footnotetext{
${ }^{1}$ In principle one can of course choose the $\tau$ for the link that will not be equal to $\mathbb{1}$ arbitrarily, for example in [72] $\tau=0$ is used. However, the choice $\tau=N_{\tau}-1$ gives the static propagator the simplest form, see equation (4.16).
} 
Then, it is enough to construct the transformation for some $\mathbf{n} \in \Lambda_{s}$ and repeat the process for all other elements of $\Lambda_{s}$.

Gauge invariance of $S_{g}$ and $S_{f}$ implies that they are unchanged if we make the local replacement

$$
U_{0}(\tau, \mathbf{n}) \rightarrow \Omega(\tau, \mathbf{n}) U_{0}(\tau, \mathbf{n}) \Omega(\tau+1, \mathbf{n})^{\dagger},
$$

for all $\tau \in \Lambda_{\tau}$, where all $\Omega$ are arbitrary $\mathrm{SU}\left(N_{c}\right)$-matrices. Specifically, if we choose $\Omega(0, \mathbf{n})=\mathbb{1}$ and $\Omega(1, \mathbf{n})=U(0, \mathbf{n}), U(0, \mathbf{n})$ will be transformed to unity:

$$
U_{0}(0, \mathbf{n}) \rightarrow \mathbb{1} U_{0}(0, \mathbf{n}) U_{0}(0, \mathbf{n})^{\dagger}=\mathbb{1} .
$$

This choice determines $\Omega(2, \mathbf{n})$, if we demand that $U(1, \mathbf{n})$ is also transformed to unity:

$$
\begin{aligned}
U_{0}(1, \mathbf{n}) & \rightarrow \Omega(1, \mathbf{n}) U_{0}(1, \mathbf{n}) \Omega(2, \mathbf{n})^{\dagger} \stackrel{!}{=} \mathbb{1} \\
& \Longrightarrow \Omega(2, \mathbf{n})=U_{0}(0, \mathbf{n}) U_{0}(1, \mathbf{n}) .
\end{aligned}
$$

Continuing this way, all $U_{0}(\tau, \mathbf{n})$ can be transformed to unity except of $U_{0}\left(N_{t}-1, \mathbf{n}\right)$, where no additional choices for $\Omega$ can be made due to the periodic boundary conditions, which imply $\Omega\left(N_{\tau}, \mathbf{n}\right)=\Omega(0, \mathbf{n})$. In total, one has: ${ }^{2}$

$$
\begin{aligned}
\Omega(\tau, \mathbf{n}) & =\prod_{\tau^{\prime}=0}^{\tau-1} U_{0}\left(\tau^{\prime}, \mathbf{n}\right), \\
U_{0}(\tau, \mathbf{n}) & \rightarrow \begin{cases}\mathbb{1}, & \text { if } \tau<N_{\tau}-1, \\
\prod_{\tau=0}^{N_{\tau}-1} U_{0}(\tau, \mathbf{n}) & \text { if } \tau=N_{\tau}-1 .\end{cases}
\end{aligned}
$$

Due to the left-invariance of the Haar measure, we can make the additional replacement

$$
U_{0}\left(N_{\tau}-1\right) \rightarrow \prod_{\tau=0}^{N_{\tau}-2} U_{0}(\tau, \mathbf{n})^{\dagger} U_{0}\left(N_{\tau}-1\right),
$$

without changing the value for $Z$, resulting in the overall transformation

$$
U_{0}(\tau, \mathbf{n}) \rightarrow \begin{cases}\mathbb{1}, & \text { if } \tau<N_{\tau}-1 \\ U_{0}\left(N_{\tau}-1, \mathbf{n}\right) & \text { if } \tau=N_{\tau}-1\end{cases}
$$

Now the integration of $U_{0}(\tau, \mathbf{n})$ is trivial for $\tau<N_{\tau}-1$ using the normalization of the Haar measure

$$
\int_{\mathrm{SU}\left(N_{c}\right)} \mathrm{d} U \mathbb{1}=\mathbb{1}
$$

The only remaining temporal link variables are $U_{0}\left(N_{\tau}-1, \mathbf{n}\right)$, which we rename to $W(\mathbf{n})$. Consequently, the path integral of the effective theory reduces to

$$
\mathrm{d}\left[U_{0}\right]=\prod_{\mathbf{n} \in \Lambda_{s}} \mathrm{~d} W(\mathbf{n})=: \mathrm{d}[W]
$$

which is obviously the measure of a 3-dimensional theory. Unless otherwise mentioned, we will assume that this gauge fixing procedure has been implemented in the following discussions.

Even after integration of the spatial links and gauge fixing there is a residual gauge invariance,

${ }^{2}$ For products of non-commutative objects we have the convention $\prod_{i=1}^{N} A_{i}=\delta(i \leq N)\left(\prod_{i=1}^{N-1} A_{i}\right) A_{N}+\delta(i>N) \mathbb{1}$. Following [96] we use the notation that for any logical statement $S, \delta(S)$ is 1 if $S$ is true and 0 if $S$ is false. 


\section{Basics}

which is inherited by the effective theory and implies that it is invariant under the local transformations

$$
W(\mathbf{n})=\Omega(\mathbf{n}) W(\mathbf{n}) \Omega(\mathbf{n})^{\dagger},
$$

where the $\Omega$ are again $\mathrm{SU}\left(N_{c}\right)$-matrices. This means that the effective theory only depends on $W(\mathbf{n})$ in terms of local traces and determinants of powers of $W(\mathbf{n})$. Determinants can be expressed in terms of traces of powers of a matrix. Furthermore, due to the Cayley-Hamilton theorem (see section 4.6.3 for details), for $W \in \mathrm{SU}(2)$ the trace $\operatorname{tr}\left(W^{n}\right)$ can be expressed in terms of $\operatorname{tr}(W)$ for all $^{3} n \in \mathbb{N}$ and for $W \in \mathrm{SU}(3)$ it can be expressed in terms of $\operatorname{tr}(W)$ and the complex conjugate $\operatorname{tr}(W)^{*}$. Therefore, $\mathrm{SU}(2)$ and $\mathrm{SU}(3)$ are special in the sense that the effective theories will depend on the Wilson line only in terms of Polyakov loops

$$
L(\mathbf{n}):=\operatorname{tr}(W(\mathbf{n})) .
$$

This is not true for $N_{c}>3$ and in these cases traces of higher powers of $W(\mathbf{n})$ (or alternatively representations of larger dimension) must occur.

\section{Strong coupling and hopping expansion}

Both the strong coupling and the hopping expansion are convergent series with a finite radius of convergence $^{4}$, for an introduction to them we refer to [95, 97, 98]. The strong coupling expansion is an expansion of the exponential containing the gauge part of the action around vanishing lattice coupling $\beta$. Instead of directly using the Taylor expansion of the exponential, a resummation of higher orders can be achieved by exploiting the fact that $S_{g, P}$ in the gauge part of the action equation (2.8) is a $\mathrm{SU}\left(N_{c}\right)$-valued class function:

$$
S_{g}[U]=\sum_{p \in P_{\Lambda}} S_{g, P}\left(U_{p}\right)=\sum_{p \in P_{\Lambda}} S_{g, P}\left(V^{-1} U_{p} V\right)
$$

where $V \in \mathrm{SU}\left(N_{c}\right)$. The Peter-Weyl theorem then tells us that one can apply non-abelian Fourier analysis to the exponential and expand it in terms of the character $\chi_{r}$ of the irreducible representations $r$ of $\mathrm{SU}\left(N_{c}\right)$ at every plaquette:

$$
\begin{aligned}
e^{-S_{g}[U]} & =\prod_{p \in P_{\Lambda}} e^{-S_{g, P}\left(U_{p}\right)} \\
& =c_{0}^{\left|P_{\Lambda}\right|} \prod_{p \in P_{\Lambda}}\left(1+\sum_{r \neq 0} d_{r} a_{r}(\beta) \chi_{r}\left(U_{p}\right)\right) .
\end{aligned}
$$

In this formula, the sum goes over all irreducible representation except of the trivial one, whose expansion coefficient $c_{0}$ has been pulled out of the product and the character of the trivial representation is simply 1. $\left|P_{\Lambda}\right|$ denotes the number of positively oriented plaquettes of the lattice and in general for any finite set $M$, we write $|M|$ for the number of elements in this set. Furthermore, $d_{r}$ corresponds to the dimension of the representation $r$ and $a_{r}(\beta)$ is the character expansion coefficient divided by $c_{0}$ :

$$
a_{r}(\beta)=\frac{c_{r}(\beta)}{c_{0}(\beta)}
$$

The expansion coefficients can be computed by utilizing the orthogonality relation of the char-

\footnotetext{
${ }^{3}$ We denote by $\mathbb{N}$ all natural numbers including 0 . For $\mathbb{N} \backslash\{0\}$ we use the notation $\mathbb{N}^{*}$.

${ }^{4}$ This is in contrast to the Feynman graph expansion around vanishing coupling in the continuum, which is an asymptotic expansion. For a discussion contrasting asymptotic and convergent expansions, see [99].
} 
acters

$$
c_{r}(\beta)=\int_{\mathrm{SU}\left(N_{c}\right)} \mathrm{d} U \chi_{r}(U)^{*} e^{-S_{g, P}\left(U_{p}\right)}
$$

and the evaluation of the integral results in [97]

$$
c_{r}(\beta)=\sum_{n=-\infty}^{\infty} \operatorname{det}_{1 \leq i, j \leq N_{c}}\left(I_{\alpha_{j}+i-j+n}\left(\beta / N_{c}\right)\right) .
$$

Here, $I_{\alpha_{j}+i-j+n}$ is a modified Bessel function of the first kind. Furthermore, the representation $r$ has been labeled by $N_{c}-1$ natural numbers $r=\left(\alpha_{1}, \alpha_{2}, \ldots, \alpha_{N_{c}}=0\right)$ with $\alpha_{1} \geq \alpha_{2} \geq \cdots \geq \alpha_{N_{c}}$. This labeling can be illustrated by the use of Young tableaux.

Truncating this expansion, the integration of the spatial links can be achieved (for pure gauge theory, to include fermions we need to combine it with the hopping expansion). For those cases, where not more than two representations share a common link, one can use the formulas

$$
\begin{aligned}
\int_{\mathrm{SU}\left(N_{c}\right)} \mathrm{d} U \chi_{r}(U V) \chi_{s}\left(W U^{-1}\right) & =\delta_{r s} \frac{1}{d_{r}} \chi_{r}(V W), \\
\int_{\mathrm{SU}\left(N_{c}\right)} \mathrm{d} U \chi_{r}\left(U V U^{-1} W\right) & =\frac{1}{d_{r}} \chi_{r}(V) \chi_{r}(W) .
\end{aligned}
$$

Note that these formulas imply that all cases, where a single non-trivial irreducible representation is at one link, vanish after integration. Using mainly these formulas, the effective theory was computed for $\mathrm{SU}(3)$ in [79] to rather high orders in the coefficient of the fundamental coefficient $u(\beta):=a_{f}(\beta) / d_{f}=a_{f}(\beta) / N_{c}$ and the analogous computations for large $N_{c}$ can be found in [94] (which, considering the integration rules above, mainly consist of replacing $d_{r}$ and $a_{r}$ by their appropriate generalizations to $N_{c}$ ). The coefficients of the higher representations can be reexpressed in terms of the fundamental character coefficient, which is always smaller than one for finite $\beta$-values, and consequently the expansion can be organized according to powers of the fundamental character.

The relation between the coefficients of higher representations and the fundamental one takes a simple form in the large $N_{c}$ limit [97], which is best show by introducing double Young diagrams, which correspond to a decomposition of a representation into complex conjugate contributions. This decomposition also allows one to express a character $\chi_{r}(U)$ in its most compact form in terms of $\operatorname{tr}\left(U^{p}\right)$ and $\operatorname{tr}\left(U^{\dagger q}\right)$ [100]. Using this expression, one can handle also those integrations, where more than two non-trivial representations share a link, since they are reduced to the integral

$$
\int_{\mathrm{SU}\left(N_{c}\right)} \mathrm{d} U U_{i_{1}, j_{1}} \cdots U_{i_{p}, j_{p}} U_{k_{1}, l_{1}}^{\dagger} \cdots U_{k_{q}, l_{q}}^{\dagger} \cdot
$$

This integral has been discussed extensively in the literature [55, 101-103] and in appendix C we give a generating function for it.

Alternatively, the cases with more than two non-trivial representations can be handled by writing the characters in terms of the representation matrices and reducing the resulting tensor product into irreducible representations. In the resulting Clebsch-Gordan series, contributions from non-trivial representations vanish after integration. This obviously also gives a selection rule for the integrals that have to be considered, which are of course reflected in the special cases equations (2.44) and (2.45). The necessary integrals for the character expansion of SU(2) Yang-Mills theory in 3 dimensions have been computed using this method in [50].

Previously $[65,73,74]$, the hopping expansion was done by expanding the fermion determinant that arises after the integration of the fermion fields around $\kappa^{(f)}=0$, i. e. the heavy quark limit. More precisely, since the combination of $\kappa^{(f)}$ and $e^{ \pm a \mu^{(f)}}$ in equation (2.12) can become large even 
for small $\kappa^{(f)}$, one formally distinguishes those $\kappa^{(f)}$ which are multiplied by $e^{ \pm a \mu^{(f)}}$ from those which are not, and only expands with respect to those $\kappa^{(f)}$, which are not multiplied by $e^{ \pm a \mu^{(f)}}$. To this end, it is useful to split the quark matrix $Q$ that occurs in equation (2.12) in the form $S_{f}[U, \Psi, \bar{\Psi}]=-\bar{\Psi} Q \Psi$ into temporal and spatial hopping matrices, where the temporal hopping matrix $T$ contains the dependence on chemical potential.

$$
\begin{aligned}
Q & =-1+T+S, \\
\operatorname{det}(Q) & =\operatorname{det}(-1+T) \operatorname{det}\left(-1+(-1+T)^{-1} S\right) \\
& =\operatorname{det}\left(Q_{\text {stat }}\right) \operatorname{det}\left(Q_{\text {kin }}\right) .
\end{aligned}
$$

The determinant and the inverse of $Q_{\text {stat }}$ are explicitly known. Therefore, when the hopping expansion is then done for the determinant of $Q_{\text {kin }}$ using the formal identity

$$
\operatorname{det}\left(Q_{\text {kin }}\right)=\exp \left(\operatorname{tr}\left(\log \left(Q_{\text {kin }}\right)\right)\right)
$$

one obtains an expansion in spatial hops, where every spatial hop is proportional to the hopping parameter $\kappa^{(f)}$, but also contains a resummation of all temporal windings, which contain $\kappa^{(f)} e^{ \pm a \mu^{(f)}}$. In this way, the complete dependence on chemical potential in each order of the hopping expansion is included. As we will introduce an alternative method to organize the hopping expansion, we refer the interested reader to the above-mentioned references for further details.

The effective action obtained in this way has the following general structure

$$
\begin{aligned}
S_{\mathrm{eff}}= & S^{s}[W]\left(\left\{\lambda_{i}\left(\beta, \kappa^{(f)}, N_{\tau}\right)\right\}_{1 \leq i<\infty}\right) \\
& +S^{a}[W]\left(h_{1}\left(\beta, \kappa^{(f)}, \mu^{(f)}, N_{\tau}\right), \bar{h}_{1}\left(\beta, \kappa^{(f)}, \mu^{(f)}, N_{\tau}\right),\left\{h_{i}\left(\beta, \kappa^{(f)}, N_{\tau}\right)\right\}_{2 \leq i<\infty}\right) .
\end{aligned}
$$

In this equation, we indicated sets of effective couplings which depend on the parameters of the original theory. Of these couplings, the $\lambda_{i}$ are the effective couplings of the $Z\left(N_{c}\right)$-symmetric part of the action $S^{s}$, that is invariant under the global transformation

$$
L(\mathbf{n}) \rightarrow z L(\mathbf{n}) \text { for all } \mathbf{n} \in \Lambda_{s} \text { and } z \in Z\left(N_{c}\right)=\left\{e^{i \frac{n \pi}{N_{c}}} \mid n \in \mathbb{N} \text { and } 0 \leq n<N_{c}-1\right\},
$$

whereas the $h_{i}$ multiply the symmetry breaking terms in $S^{a}$. Note that the symmetry breaking terms are due to the fermion determinant, but the $\lambda_{i}$ also depend on $\kappa$, as the fermion determinant also contains center symmetric contributions. Of the couplings for the asymmetric terms, $h_{1}^{(f)} / \bar{h}_{1}^{(f)}$ multiply every quark/antiquark temporal winding and encode the dependence on chemical potential of the effective theory. In this notation, the resummation of temporal windings that we mentioned above corresponds to a resummation in $h_{1}^{(f)} / \bar{h}_{1}^{(f)}$.

Except of these couplings, all other couplings are small in the range of validity of the expansions, which justifies the application of series expansion methods for the evaluation of the effective theory. As the integrations can be done exactly in the case of series expansions, they naturally circumvent the sign problem. The application of series expansion methods are also attractive from another point of view: since we use series expansion methods to derive the effective theory, successfully applying these methods to evaluate the effective theory (which can also be evaluated by numerical methods, serving as a crosscheck) also gives justification for the derivation of the effective theory. Furthermore, some diagrammatic methods that are used for the evaluation of the effective theory can also teach useful lessons for the derivation of the theory and vice versa (see for example section 4.6). In this way a useful synergy between the derivation and evaluation of the theory can be achieved. Additionally, the numerical methods for determining the effective theory in [92] also 


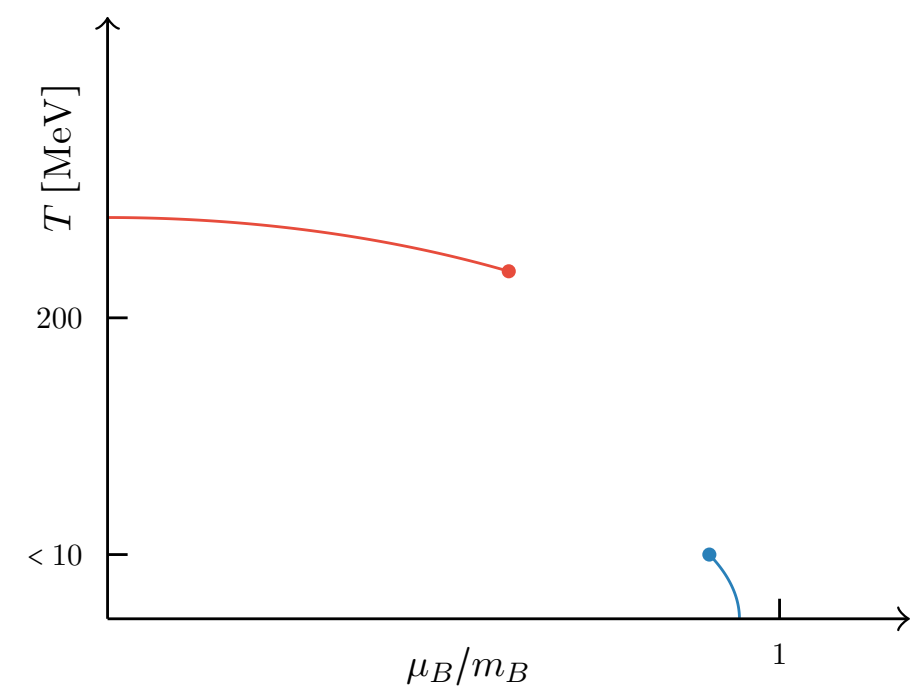

Figure 2.1: Taken from [73]. The phase diagram of QCD with very heavy quarks.

employ series expansion methods. Before we take a closer look at the evaluation of the effective theory using series expansion methods, we will mention some results obtained from the effective theory.

\subsubsection{Results from the effective theory}

In this section will only consider the cases $N_{c}=2$ and $N_{c}=3$, the changes that occur when large $N_{c}$ is considered will be discussed in chapter 6 . In figure 2.1 a schematic version of the phase diagram of QCD with heavy quarks in the temperature-baryon chemical potential $\left(T, \mu_{B}\right)$ plane is shown. Infinite quark mass is equivalent to pure gauge theory (the upper right corner of the Columbia plot), which has has a first-order deconfinement transition for $N_{c}=3$, signaled by a spontaneous breakdown of the center symmetry. Finite quark masses introduce terms $\propto \kappa \propto 1 / m_{q}$ which explicitly break center symmetry. Consequently, for sufficiently heavy quarks the transition is still first order, but gets weaker for decreasing masses until it ends at a critical second-order point for some critical mass $m_{q, c}$ which is of the universality class $Z(2)$.

In the effective theory, this symmetry breaking pattern is correctly reproduced. Using the methods mentioned in section 2.1.1, one can obtain a set of critical couplings $\lambda_{i, c}=\lambda_{i}\left(\beta_{c}, \kappa_{c}^{(f)}, N_{\tau}\right)$, $h_{i, c}=h_{i}\left(\beta_{c}, \kappa_{c}, N_{\tau}\right)\left(\mu=0\right.$ means $\left.h_{1}=\bar{h}_{1}\right)$. Inverting these relations results in predictions for $\beta_{c}\left(N_{\tau}\right)$ and $\kappa_{c}\left(N_{\tau}\right)$, which in turn can be compared to full QCD. For $\mathrm{SU}(2)$ and $\mathrm{SU}(3)$ pure gauge theory, the simplest (logarithmic-resummed) effective theory with only the first nearest neighbor coupling correctly predicts the order of the transition and the associated $\beta_{c}\left(N_{\tau}\right)$ are within $10 \%$ of the values from simulations of full Yang-Mills theory on lattices with $N_{\tau}=2, \ldots, 16$ [79]. Furthermore, including heavy quarks up to the $\kappa^{2}$ corrections, a second-order endpoint is observed for $N_{c}=3$ and the corresponding $\kappa_{c}$ is within $10 \%$ of the value predicted by full QCD simulations with $N_{\tau}=4$ [61]. As we stressed already, the sign problem can be dealt with in the effective theory and it can be simulated at finite density. Therefore, the critical endpoint can be determined both as a function of chemical potential and quark mass [61], and the qualitative behavior when increasing the chemical potential is that of figure 2.1. This qualitative behavior agrees with predictions from continuum studies employing a Polyakov loop model [104] or the functional renormalization group approach [105]. 
Going parallel to the baryon chemical potential axis at low temperature there is the onset transition to condensed baryon matter. From simple statistical physics arguments combined with phenomenology (see section section 5.1), it is clear that at $T=0$ the nuclear liquid gas transition is of first order. Increasing the temperature, this transition also ends in a second-order endpoint. The endpoint moves to lower temperatures when the quark mass is increased. This can be understood from the fact that the binding energy per baryon, which sets the scale of the scale for the critical endpoint, is reduced (exponentially) according to nuclear physics Yukawa potentials with meson exchange. The phenomenological properties leading to the critical properties of the liquid gas transition can be observed in rather simple calculations using the effective theory at strong coupling [65] and we will review those critically in chapter 5. In simulations of the effective theory including $\kappa^{4}$ corrections, a first-order transition ending at some finite temperature is explicitly seen [65]. Including gauge corrections and sufficiently high orders, the lattice spacing can be varied and the continuum approach can be studied. Using corrections up to $u^{5} \kappa^{8}$, continuum-like behavior was obtained immediately after the onset transition. This was possible for rather heavy quarks only, and a stable continuum extrapolation for quarks with sufficiently light masses such that a critical line for the onset is observed is currently out of reach.

Before we finally discuss the linked cluster expansion of the simplest Polyakov loop theory, we introduce a technical device that is useful both for the derivation and evaluation of the effective theory.

\subsection{Polymer expansions}

Polymer expansions in their abstract formulation are the underlying principle of many series expansions in physics, the high temperature (weak graph) expansion of the Ising model being only one example. They also have been used in the derivation of the effective theory [79]. Depending on the context of their application and also the form of their formulation, they are also called cluster expansions [106] or method of moments and cumulants [98, 107, 108]. Some of the earliest forms and applications of these expansions can be found in [109-112]. They have been studied rigorously in mathematical physics, see [113] and references therein and [114] for an overview of the developments since then. As can be seen from the above mentioned references, these expansions have a long and rich history and are still the subject of current publications, so our account of the literature is far from exhaustive.

Given the partition function $Z$ of a system, the polymer expansion formula gives an algebraic identity for $\log (Z)$. Here, we will only be concerned with algebraic properties of this identity, so both $Z$ and $\log (Z)$ can be considered formal power series. Concerning convergence properties we refer the interested reader to the literature. In general we will always be more concerned with the generation of series coefficients and will only determine the convergence using more heuristic methods like the ratio test or Padé approximants.

The motivation for this section is two-fold: one is to introduce some useful notations and definitions. The other is that we want to highlight the fact that some aspects of the polymer expansions can be simplified by noting that they are actually Taylor expansions of the logarithm of the partition function. This was already pointed out in [115], but it was Dobrushin [116] who made full use of this fact, replacing combinatorial definitions by analytical ones, and this was further explored in, for example, [117]. Our notation is inspired by [99], but we show a slightly more general form of the expansion formula first, which is necessary to apply it to the free graph expansion of section 3.2. In this form the theorem corresponds to the version in, for example, [107, 108], but we will avoid, as mentioned above, dealing with the combinatorics of the moment-cumulant relations.

The general principle of polymer expansions can be formulated without reference to a specific physical system, explaining the wide range of applicability. As a preparatory step we derive a useful 
formula concerning sums over sets.

\subsubsection{Sums over disjoint subsets}

Let $M$ be a countable set and $M_{1}$ and $M_{2}$ a partition of $M$, which means that $M_{1}$ and $M_{2}$ are disjoint and their union is $M$. Furthermore we assume that there is a function

$$
f: M^{n} \rightarrow \mathbb{C}
$$

with $n \in \mathbb{N}^{*}$.

Consider the following sum

$$
S=\sum_{\gamma_{1}, \ldots, \gamma_{n} \in M} f\left(\gamma_{1}, \ldots, \gamma_{n}\right)=\sum_{\gamma_{1}, \ldots, \gamma_{n} \in M_{1} \cup M_{2}} f\left(\gamma_{1}, \ldots, \gamma_{n}\right) .
$$

We want to rewrite this into a sum where the elements of $M_{1}$ and $M_{2}$ are indicated separately. A first try could be

$$
\sum_{k+l=n} \sum_{\gamma_{1}, \ldots, \gamma_{k} \in M_{1}} \sum_{\gamma_{k+1}, \ldots, \gamma_{k+l} \in M_{2}} f\left(\gamma_{1}, \ldots, \gamma_{k}, \gamma_{k+1}, \ldots, \gamma_{k+l}\right)
$$

where we assume the convention that

$$
\sum_{\gamma_{1}, \ldots, \gamma_{k}}=1 \text { if } k=0
$$

Obviously, this does not work because in the original sum the arguments of $f$ are not arranged in a way that the elements of $M_{1}$ appear before the elements of $M_{2}$. To repair this, we introduce additional permutations

$$
\sum_{k+l=n} \sum_{\gamma_{1}, \ldots, \gamma_{k} \in M_{1}} \sum_{\gamma_{k+1}, \ldots, \gamma_{k+l} \in M_{2}} \sum_{\sigma \in S_{n}} f\left(\gamma_{\sigma(1)}, \ldots, \gamma_{\sigma(k)}, \gamma_{\sigma(k+1)}, \ldots, \gamma_{\sigma(k+l)}\right),
$$

where $S_{n}$ denotes the symmetric group of order $n$. In this formula however, the permutations which only permute the elements of $M_{1}$ and $M_{2}$ separately lead to an overcounting and the correction of this overcounting finally gives

$$
S=\sum_{k+l=n} \sum_{\gamma_{1}, \ldots, \gamma_{k} \in M_{1}} \sum_{\gamma_{k+1}, \ldots, \gamma_{k+l} \in M_{2}} \sum_{\sigma \in S_{n}} \frac{f\left(\gamma_{\sigma(1)}, \ldots, \gamma_{\sigma(k)}, \gamma_{\sigma(k+1)}, \ldots, \gamma_{\sigma(k+l)}\right)}{k ! l !} .
$$

While this representation of $S$ seems more cumbersome, it will proof useful later. We are now ready to derive the polymer expansion formula.

\subsubsection{The polymer expansion formula}

To state the content of this formula, we need to make some definitions. We start by introducing a countable set $P$ whose elements are called polymers. Furthermore it is assumed that there is a sequence of symmetric functions

$$
m_{n}: P^{n} \rightarrow \mathbb{C}
$$

which we call polymer moments. We simply write $m$ instead of $m_{n}$, when $n$ can be inferred from the number of arguments. Next, we assign weights $\varphi$ to each polymer, where $\varphi$ is a mapping from $P$ to $\mathbb{C}$. 


\section{Basics}

Using these definitions, the partition function $Z(P)$ associated with the polymer set $P$ is defined to be a power series in the weights

$$
\begin{aligned}
Z(P) & =1+\sum_{n=1}^{\infty} \sum_{\gamma_{1}, \ldots, \gamma_{n} \in P} \frac{1}{n !} m\left(\gamma_{1}, \ldots, \gamma_{n}\right) \varphi\left(\gamma_{1}\right) \cdots \varphi\left(\gamma_{n}\right) \\
& =\sum_{n=0}^{\infty} \sum_{\gamma_{1}, \ldots, \gamma_{n} \in P} \frac{1}{n !} m\left(\gamma_{1}, \ldots, \gamma_{n}\right) \varphi\left(\gamma_{1}\right) \cdots \varphi\left(\gamma_{n}\right) .
\end{aligned}
$$

The polymer expansion formula now states that

$$
\log (Z(P))=\sum_{n=1}^{\infty} \sum_{\gamma_{1}, \ldots, \gamma_{n} \in P} \frac{1}{n !} c\left(\gamma_{1}, \ldots, \gamma_{n}\right) \varphi\left(\gamma_{1}\right) \cdots \varphi\left(\gamma_{n}\right)
$$

where $c\left(\gamma_{1}, \ldots, \gamma_{n}\right)$ is a rational coefficient with the property that it vanishes, if its arguments can be partitioned into two groups $\alpha_{1}, \ldots, \alpha_{k}$ and $\beta_{1}, \ldots, \beta_{l}$ such that $m$ factorizes over these two groups: $m\left(\gamma_{1}, \ldots, \gamma_{\tilde{n}}\right)=m\left(\alpha_{1}, \ldots, \alpha_{\tilde{k}}, \beta_{1}, \ldots, \beta_{\tilde{l}}\right)=m\left(\alpha_{1}, \ldots, \alpha_{\tilde{k}}\right) m\left(\beta_{1}, \ldots, \beta_{\tilde{l}}\right)$ for any $\tilde{n} \leq n$ (and analogously for $l$ and $k$ ). We remind the reader that $m$ is defined to be symmetric. The $c$ are called polymer cumulants.

What makes the polymer expansion useful is that the vanishing of the coefficient for certain combinations of the elements of $P$ usually simplifies the combinatorics of the expansion at hand.

Considering the Taylor expansion of $\log (Z(P))$ in terms of the polymer weights, one obtains

$$
\log (Z(P))=\sum_{n=1}^{|P|} \sum_{\left\{\gamma_{1}, \ldots, \gamma_{n}\right\} \in \mathcal{S}_{n}(P)} \sum_{k_{1}, \ldots, k_{n} \in \mathbb{N}^{*}} C(\underbrace{\gamma_{1}, \ldots, \gamma_{1}}_{k_{1} \text {-times }}, \ldots, \underbrace{\gamma_{n}, \ldots, \gamma_{n}}_{k_{n} \text {-times }}) \varphi\left(\gamma_{1}\right)^{k_{1}} \cdots \varphi\left(\gamma_{n}\right)^{k_{n}},
$$

where $\mathcal{S}_{n}(P)$ denotes the set of subsets of $P$ with $n$ elements and the coefficient of the expansion reads

$$
C(\underbrace{\gamma_{1}, \ldots, \gamma_{1}}_{k_{1} \text {-times }}, \ldots, \underbrace{\gamma_{n}, \ldots, \gamma_{n}}_{k_{n} \text {-times }})=\left.\frac{1}{k_{1} ! \cdots k_{n} !} \frac{\partial^{k_{1}+\cdots+k_{n}} \log (Z(\mathcal{P}))}{\partial \varphi\left(\gamma_{1}\right)^{k_{1}} \ldots \partial \varphi\left(\gamma_{n}\right)^{k_{n}}}\right|_{\varphi\left(\gamma_{i}\right)=0}=: C_{\left(\gamma_{1}, k_{1}\right), \ldots,\left(\gamma_{n}, k_{n}\right)} .
$$

To get to equation (2.62), one replaces the sum over the subsets $\mathcal{S}_{N}(P)$ and multiplicities $k_{1}, \ldots, k_{n}$ by independent sums over $P$. This leads to an overcounting, which is corrected by dividing by the number of unique permutations of $(\underbrace{\gamma_{1}, \ldots, \gamma_{1}}_{k_{1} \text {-times }}, \ldots, \underbrace{\gamma_{n}, \ldots, \gamma_{n}}_{k_{n} \text {-times }})$, of which there are

$$
\frac{\left(k_{1}+\cdots+k_{n}\right) !}{k_{1} ! \cdots k_{n} !} .
$$

This means that the coefficient $c$ in equation (2.62) reads

$$
c\left(\gamma_{1}, \ldots, \gamma_{n}\right)=\left.\frac{\partial^{n} \log (Z(P))}{\partial \varphi\left(\gamma_{1}\right) \cdots \partial \varphi\left(\gamma_{n}\right)}\right|_{\varphi\left(\gamma_{i}\right)=0} .
$$

The only thing that remains to be shown is that $c\left(\gamma_{1}, \ldots, \gamma_{n}\right)$ vanishes if its arguments can be partitioned in the aforementioned way. Consider the set $S$ consisting of the $\gamma_{1}, \ldots, \gamma_{n}$ (without multiplicities). $S \subset P$ itself is a set of polymers and to compute $c\left(\gamma_{1}, \ldots, \gamma_{n}\right)$, it is enough to 
consider only $Z(S)$ in equation (2.67). Furthermore, $S$ can be partitioned into two non-empty and disjoint sets $S_{1}$ and $S_{2}$ such that

$$
m\left(\alpha_{1}, \ldots, \alpha_{k}, \beta_{1}, \ldots, \beta_{l}\right)=m\left(\alpha_{1}, \ldots, \alpha_{k}\right) m\left(\beta_{1}, \ldots, \beta_{l}\right)
$$

if $\alpha_{1}, \ldots, \alpha_{k} \in S_{1}$ and $\beta_{1}, \ldots, \beta_{l} \in S_{2}$. With the help of equation (2.58) and the symmetry of $m$ this implies that not only $m$, but also $Z(S)$ factorizes:

$$
\begin{aligned}
& Z(S)=Z\left(S_{1} \cup S_{2}\right) \\
& =\sum_{n=0}^{\infty} \sum_{\gamma_{1}, \ldots, \gamma_{n} \in S_{1} \cup S_{2}} \frac{1}{n !} m\left(\gamma_{1}, \ldots, \gamma_{n}\right) \varphi\left(\gamma_{1}\right) \cdots \varphi\left(\gamma_{n}\right) \\
& \stackrel{(2.58)}{=} \sum_{n=0}^{\infty} \sum_{k+l=n} \sum_{\gamma_{1}, \ldots, \gamma_{k} \in S_{1}} \sum_{\gamma_{k+1}, \ldots, \gamma_{k+l} \in S_{2}} \sum_{\sigma \in S_{n}} \frac{1}{n ! k ! l !} m\left(\gamma_{\sigma(1)}, \ldots, \gamma_{\sigma(k)}, \gamma_{\sigma(k+1)}, \ldots, \gamma_{\sigma(k+l)}\right) \\
& \times \varphi\left(\gamma_{\sigma(1)}\right) \cdots \varphi\left(\gamma_{\sigma(n)}\right) \\
& =\sum_{n=0}^{\infty} \sum_{k+l=n} \sum_{\gamma_{1}, \ldots, \gamma_{k} \in S_{1}} \sum_{\gamma_{k+1}, \ldots, \gamma_{k+l} \in S_{2}} \frac{1}{k ! l !} m\left(\gamma_{1}, \ldots, \gamma_{k}, \gamma_{k+1}, \ldots, \gamma_{k+l}\right) \varphi\left(\gamma_{1}\right) \cdots \varphi\left(\gamma_{n}\right) \\
& =\sum_{n=0}^{\infty} \sum_{k+l=n} \sum_{\gamma_{1}, \ldots, \gamma_{k} \in S_{1}} \sum_{\gamma_{k+1}, \ldots, \gamma_{k+l} \in S_{2}} \frac{1}{k ! l !} m\left(\gamma_{1}, \ldots, \gamma_{k}\right) m\left(\gamma_{k+1}, \ldots, \gamma_{k+l}\right) \\
& \times \varphi\left(\gamma_{1}\right) \cdots \varphi\left(\gamma_{k}\right) \varphi\left(\gamma_{k+1}\right) \cdots \varphi\left(\gamma_{n}\right) \\
& =\left(\sum_{k=0}^{\infty} \sum_{\gamma_{1}, \ldots, \gamma_{k} \in S_{1}} \frac{1}{k !} m\left(\gamma_{1}, \ldots, \gamma_{k}\right) \varphi\left(\gamma_{1}\right) \cdots \varphi\left(\gamma_{k}\right)\right) \\
& \times\left(\sum_{l=0}^{\infty} \sum_{\gamma_{1}, \ldots, \gamma_{l} \in S_{2}} \frac{1}{l !} m\left(\gamma_{1}, \ldots, \gamma_{l}\right) \varphi\left(\gamma_{1}\right) \cdots \varphi\left(\gamma_{l}\right)\right) \\
& =Z\left(S_{1}\right) Z\left(S_{2}\right) \text {. }
\end{aligned}
$$

Consequently

$$
\log (Z(S))=\log \left(Z\left(S_{1}\right)\right)+\log \left(Z\left(S_{2}\right)\right)
$$

and since neither $\log \left(Z\left(S_{1}\right)\right)$ nor $\log \left(Z\left(S_{2}\right)\right)$ depend on all of the $\varphi\left(\gamma_{1}\right), \ldots, \varphi\left(\gamma_{n}\right)$ the coefficient $c\left(\gamma_{1}, \ldots, \gamma_{n}\right)$ vanishes according to equation (2.67), as was claimed.

If there is a binary symmetric relation, which decides if two polymers are to be regarded linked or disjoint, a useful corollary can be obtained. We introduce the notation $\gamma_{i} \cap \gamma_{j} \neq \emptyset$ if the polymers are linked and $\gamma_{i} \cap \gamma_{j}=\emptyset$ when they are disjoint. It is always assumed that $\gamma_{i} \cap \gamma_{i} \neq \emptyset$. The meaning of $\cap$ here is not necessarily that of an intersection of sets and the meaning of being linked or disjoint depends on the details of the application of the polymer expansion. For now it is enough that such a relation between the polymers exists. Additionally, the sets $\mathcal{D}_{n}(P)$ are introduced, which consist of all subsets of $P$ with $n$ elements and pairwise disjoint polymers. The union of these sets is denoted by $\mathcal{D}(P)=\cup_{n} \mathcal{D}_{n}(P)$.

Suppose now that the partition function reads

$$
Z(P)=1+\sum_{n=1}^{|P|} \sum_{\left\{\gamma_{1}, \ldots, \gamma_{n}\right\} \in \mathcal{D}_{n}(P)} \varphi\left(\gamma_{1}\right) \cdots \varphi\left(\gamma_{n}\right) .
$$

One might wonder why $Z(P)$ is defined here without a factor $1 / n$ ! in contrast to before and also 


\section{Basics}

in contrast to, for example [99]. Due to this, we stress that we defined $\mathcal{D}_{k}$ to consist of subsets of $P$ and not of tuples of elements of $P$.

If we define

$$
m\left(\gamma_{1}, \ldots, \gamma_{n}\right)= \begin{cases}1, & \text { if the arguments are pairwise disjoint } \\ 0, & \text { otherwise }\end{cases}
$$

then $Z(P)$ can be rewritten to

$$
\begin{aligned}
Z(P) & =1+\sum_{n=1}^{|P|} \sum_{\left\{\gamma_{1}, \ldots, \gamma_{n}\right\} \in \mathcal{D}_{n}(P)} m\left(\gamma_{1}, \ldots, \gamma_{n}\right) \varphi\left(\gamma_{1}\right) \cdots \varphi\left(\gamma_{n}\right) \\
& =1+\sum_{n=1}^{\infty} \sum_{\gamma_{1}, \ldots, \gamma_{n} \in P} \frac{1}{n !} m\left(\gamma_{1}, \ldots, \gamma_{n}\right) \varphi\left(\gamma_{1}\right) \cdots \varphi\left(\gamma_{n}\right)
\end{aligned}
$$

$m$ factorizes over disjoint polymers, so applying the polymer expansion formula results in

$$
\log (Z(P))=\sum_{n=1}^{|P|} \sum_{\left\{\gamma_{1}, \ldots, \gamma_{n}\right\} \in \mathcal{C}_{k}(P)} \sum_{\left(k_{1}, \ldots, k_{n}\right) \in\left(\mathbb{N}^{*}\right)^{n}} C_{\left(\gamma_{1}, k_{1}\right), \ldots,\left(\gamma_{n}, k_{n}\right)} \varphi\left(\gamma_{1}\right)^{k_{1}} \cdots \varphi\left(\gamma_{n}\right)^{k_{n}}
$$

where $C_{\left(\gamma_{1}, k_{1}\right), \ldots,\left(\gamma_{n}, k_{n}\right)}$ is the coefficient

$$
C_{\left(\gamma_{1}, k_{1}\right), \ldots,\left(\gamma_{n}, k_{n}\right)}=\left.\frac{1}{k_{1} ! \cdots k_{n} !} \frac{\partial^{k_{1}+\cdots+k_{n}} \log (Z(\mathcal{P}))}{\partial \varphi\left(\gamma_{1}\right)^{k_{1}} \cdots \partial \varphi\left(\gamma_{n}\right)^{k_{n}}}\right|_{\varphi\left(\gamma_{i}\right)=0}
$$

and $\mathcal{C}_{n}(P)$ is the set of subsets $C \subset P$ which have $n$ elements and the property that for any pair $\gamma_{1}, \gamma_{2} \in C$ there is always a sequence of polymers $\gamma_{k_{1}}, \ldots, \gamma_{k_{m}}$ in $C$ that fulfills $\gamma_{k_{1}}=\gamma_{1}, \gamma_{k_{m}}=\gamma_{2}$ and $\gamma_{k_{i}} \cap \gamma_{k_{i+1}} \neq \emptyset$. In other words, if we assign to every subset $M$ of $P$ the graph with vertices consisting of the polymers in $M$ and edges chosen such that linked polymers are adjacent, then only connected graphs with $n$ edges are assigned to the elements of $\mathcal{C}_{n}(P)$.

The result can be roughly summarized by saying that the expansion of the logarithm leads to clusters of linked polymers. Applied to physical models, the resulting expansions are therefore sometimes called linked cluster expansions. Confusingly, since the polymer expansion formula is quite general and enters different expansion schemes in varied ways, the name linked cluster expansion is attributed to rather different expansion schemes.

We will illustrate the difference of two expansion schemes by applying both methods to the $\mathrm{SU}(3)$ spin model. 


\section{Series expansion of the $S U(3)$ spin model}

The SU(3) spin model is a 3-dimensional effective Polyakov loop action which has the grandcanonical partition function

$$
Z(\tau, \kappa, \mu)=\int \mathrm{d}[W] e^{-S[W]},
$$

where the action $S[W]$ is that of equation (2.1), which we reproduce here for the convenience of the reader

$$
S_{\text {eff }}=-\sum_{\mathbf{n} \in \Lambda_{s}}\left(\tau \sum_{k=1}^{3}\left[L(\mathbf{n}) L^{*}\left(\mathbf{n}+\mathbf{e}_{k}\right)+L^{*}(\mathbf{n}) L\left(\mathbf{n}+\mathbf{e}_{k}\right)\right]+\kappa e^{\mu} L(\mathbf{n})+\kappa e^{-\mu} L^{*}(\mathbf{n})\right) .
$$

We also define

$$
\eta=\kappa e^{\mu}, \quad \bar{\eta}=\kappa e^{\mu},
$$

and in this way emphasize the analogy to spin models like the Ising model, but in our case we deal with spin variables $L(\mathbf{n})$ which are continuous and complex valued. In this analogy, $\langle L\rangle$ then plays the role of magnetization and $\eta$ and $\bar{\eta}$ correspond to the symmetry breaking external fields

As this model is obtained from the leading orders of both the expansions outlined in section 2.1.2 and neither includes the resummation of temporal hoppings explained in that section, nor the logarithmic resummations which have proven important in studies of the effective theory [118], it certainly is a rather crude approximation to QCD. Nevertheless, it shares important symmetries with QCD. For $\kappa=0$, the action has the center symmetry of Yang-Mills theory and the influence of the symmetry breaking terms is just like in section 2.1.3. Also, the Roberge-Weiss symmetry of QCD [119] for complex chemical potential is easily recognized in the spin model on the basis of the invariance of the Haar measure:

$$
Z\left(\tau, \kappa, i \mu_{i}\right)=Z\left(\tau, \kappa, i\left(\mu_{i}+2 \pi n / 3\right)\right) \text { for } n \in \mathbb{N} \text { and } \mu_{i} \in \mathbb{R} .
$$

Furthermore, the spin model is the simplest Polyakov loop effective theory with a sign problem for finite chemical potential and has been successfully evaluated with the methods mentioned in section 2.1.1. Consequently, its phase diagram is known and we show a schematic version in figure 3.1. Therefore, it is a natural candidate to test any new methods for evaluating effective Polyakov loop methods, which we will do here for the case of series expansion methods.

As mentioned in section 2.2, we will contrast two expansion methods. Both methods are a systematic way to derive the Taylor expansion of the free energy density in the thermodynamic limit

$$
f=-\lim _{N_{s} \rightarrow \infty} \frac{\log (Z)}{N_{s}} .
$$

The expansion can be expressed in terms of connected graphs for both cases, but the rules associated with the graphs are quite different. The necessary graph-theoretic notions are collected in appendix B, and we will make use of them from now on without further explanations. 


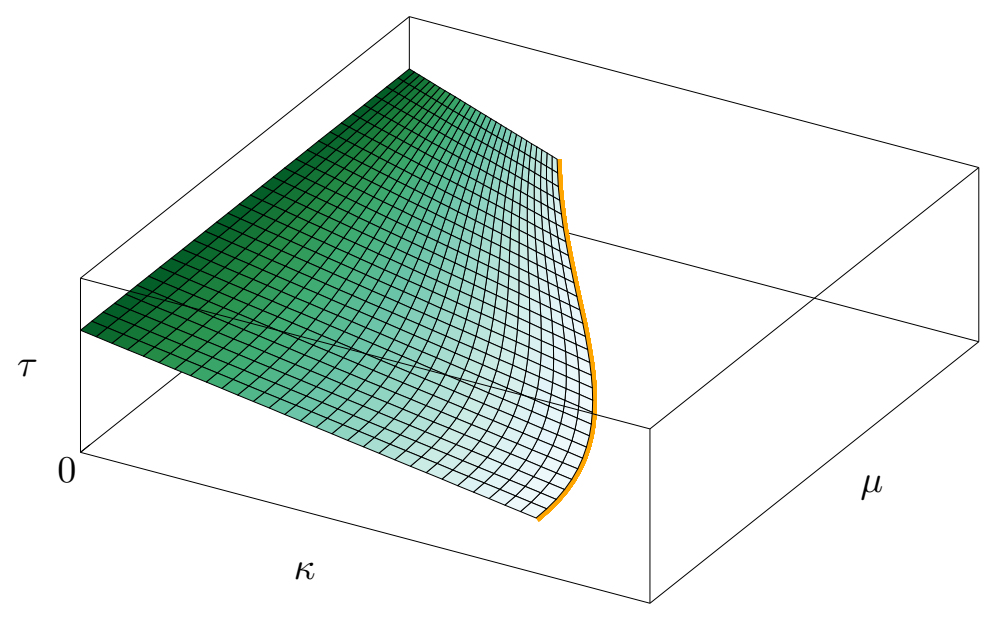

Figure 3.1: Schematic phase diagram of the $\mathrm{SU}(3)$ spin model. A surface of first-order transitions is bounded by a $Z(2)$-critical line beyond which the transitions are analytic crossovers.

\subsection{Weak graph expansion of the spin model}

The version of the weak graph expansion of the spin model we derive here is a relatively straightforward application of the polymer expansion corollary equation (2.81). A typical example of the application of the weak graph expansion is the spin-1/2 Ising model, for an introduction see [120-122]. This form of the weak expansion is a bit unnatural for the spin-model and the free graph expansion of the next section is more convenient. Nevertheless, the weak graph expansion is worthwhile to explore it here. First, a modification of the scheme explored here will be applied in chapter 4. As the model here is much simpler, it can serve as an introduction to the more complicated calculations in chapter 4. Moreover, it is natural to apply this approach to effective Polyakov loop actions which have been logarithmically resummed and we will discuss these actions in section 4.4. Still, if the reader is interested only in the free graph expansion of the spin model, he can skip this section.

The first step is to bring the partition function of the spin model to the form of equation (2.60). To this end, we use the multiplicative property of the exponential function to rewrite the partition function

$$
\begin{aligned}
Z & =\int \mathrm{d}[W] \exp \left\{\sum_{\mathbf{n} \in \Lambda_{s}}\left(\tau \sum_{k=1}^{3}\left[L(\mathbf{n}) L^{*}\left(\mathbf{n}+\mathbf{e}_{k}\right)+L^{*}(\mathbf{n}) L\left(\mathbf{n}+\mathbf{e}_{k}\right)\right]+\eta L(\mathbf{n})+\bar{\eta} L^{*}(\mathbf{n})\right)\right\} \\
& \left.=\int \mathrm{d}[W] \prod_{\mathbf{n} \in \Lambda_{s}} \prod_{k=1}^{3} \exp \left\{\tau\left[L(\mathbf{n}) L^{*}\left(\mathbf{n}+\mathbf{e}_{k}\right)+L^{*}(\mathbf{n}) L\left(\mathbf{n}+\mathbf{e}_{k}\right)\right]\right\} \prod_{\mathbf{n} \in \Lambda_{s}} \exp \left\{\eta L(\mathbf{n})+\bar{\eta} L^{*}(\mathbf{n})\right)\right\} \\
& \left.=\int \mathrm{d}[W] \prod_{\mathbf{n} \in \Lambda_{s}} \prod_{k=1}^{3}\left[1+f\left(\tau, L(\mathbf{n}), L\left(\mathbf{n}+\mathbf{e}_{i}\right)\right)\right] \prod_{\mathbf{n} \in \Lambda_{s}} \exp \left\{\eta L(\mathbf{n})+\bar{\eta} L^{*}(\mathbf{n})\right)\right\},
\end{aligned}
$$


where $f$ is determined from the series expansion of exp:

$$
f\left(\tau, L(\mathbf{n}), L\left(\mathbf{n}+\mathbf{e}_{k}\right)\right)=\sum_{n=1}^{\infty} \frac{\tau^{n}}{n !}\left[L(\mathbf{n}) L^{*}\left(\mathbf{n}+\mathbf{e}_{k}\right)+L^{*}(\mathbf{n}) L\left(\mathbf{n}+\mathbf{e}_{k}\right)\right]^{n} .
$$

In order to be able to reduce the path integral to single site integrals one has to expand the product

$$
\prod_{\mathbf{n} \in \Lambda_{s}} \prod_{k=1}^{3}\left[1+f\left(\tau, L(\mathbf{n}), L\left(\mathbf{n}+\mathbf{e}_{k}\right)\right)\right] .
$$

This expansion can be represented in terms of graphs in the following way: the lattice $\Lambda_{s}$ can be interpreted as an undirected graph $G_{\Lambda_{s}}$ by taking all elements of $\Lambda_{s}$ as vertices and the edges connect the nearest neighbors More precisely, the graph $G_{\Lambda_{s}}$ has the vertices

$$
V_{G_{\Lambda_{s}}}=\Lambda_{s}
$$

edges

$$
E_{G_{\Lambda_{s}}}=\left\{(\mathbf{n}, k) \mid \mathbf{n} \in \Lambda_{s}, k \in\{1,2,3\}\right\},
$$

and the edge-to-endpoint function

$$
\sigma_{G_{\Lambda_{s}}}((\mathbf{n}, k))=\left\{\mathbf{n},\left(\mathbf{n}+\mathbf{e}_{k}\right) \bmod \mathbf{L}\right\},
$$

where we introduced the notation $\mathbf{L}=\left(\sqrt[3]{N_{s}}, \sqrt[3]{N_{s}}, \sqrt[3]{N_{s}}\right)$. Now,

$$
\begin{aligned}
\prod_{\mathbf{n} \in \Lambda_{s}} & \prod_{k=1}^{3}\left[1+f\left(\tau, L(\mathbf{n}), L\left(\mathbf{n}+\mathbf{e}_{k}\right)\right)\right] \\
= & \prod_{(\mathbf{n}, k) \in E_{G_{\Lambda_{s}}}}\left[1+f\left(\tau, L(\mathbf{n}), L\left(\mathbf{n}+\mathbf{e}_{k}\right)\right)\right] \\
= & 1+\sum_{(\mathbf{n}, k) \in E_{G_{\Lambda_{s}}}} f\left(\tau, L(\mathbf{n}), L\left(\mathbf{n}+\mathbf{e}_{k}\right)\right) \\
& +\sum_{\left(\mathbf{n}_{1}, k_{1}\right) \in E_{G_{\Lambda_{s}}}} \sum_{\left(\mathbf{n}_{1}, k_{2}\right) \in E_{G_{\Lambda_{s}}} \backslash\left(\mathbf{n}_{1}, k_{1}\right)} f\left(\tau, L\left(\mathbf{n}_{1}\right), L\left(\mathbf{n}_{1}+\mathbf{e}_{k_{1}}\right)\right) f\left(\tau, L\left(\mathbf{n}_{2}\right), L\left(\mathbf{n}_{2}+\mathbf{e}_{k_{2}}\right)\right) \\
& +\ldots \sum_{\left|E_{G_{\Lambda_{s}}}\right|} \sum_{n=1}\left\{\prod_{\left\{\left(\mathbf{n}_{1}, k_{1}\right), \ldots,\left(\mathbf{n}_{n}, k_{n}\right)\right\} \in \mathcal{S}_{n}\left(E_{G_{\Lambda_{s}}}\right) l=1}^{n} f\left(\tau, L\left(\mathbf{n}_{l}\right), L\left(\mathbf{n}_{l}+\mathbf{e}_{k_{l}}\right)\right)\right. \\
= & 1+\sum_{G \in \mathcal{G}\left(G_{\Lambda_{s}}\right)} \prod_{(\mathbf{n}, k) \in E_{G}} f\left(\tau, L(\mathbf{n}), L\left(\mathbf{n}+\mathbf{e}_{i}\right)\right),
\end{aligned}
$$

where we introduced the notation that for a graph $G, \mathcal{G}(G)$ is the set of all subgraphs of $G$, with those subgraphs excluded which contain an isolated vertex. Note that for now, we do not identify isomorphic graphs and disconnected graphs are not excluded from $\mathcal{G}(G)$.

Next, we define the weight $\varphi$ of a graph $G \in \mathcal{G}\left(G_{\Lambda_{s}}\right)$ appropriately:

$$
\left.\varphi(G)=\frac{1}{z_{0}^{N_{s}}} \int \mathrm{d}[W] \prod_{\mathbf{n} \in \Lambda_{s}} \exp \left\{\eta L(\mathbf{n})+\bar{\eta} L^{*}(\mathbf{n})\right)\right\} \prod_{(\mathbf{n}, k) \in E_{G}} f\left(\tau, L(\mathbf{n}), L\left(\mathbf{n}+\mathbf{e}_{i}\right)\right)
$$




$$
\left.=\frac{1}{z_{0}^{\left|V_{G}\right|}} \int \prod_{\mathbf{n} \in V(G)} \mathrm{d} W \exp \left\{\eta L(\mathbf{n})+\bar{\eta} L^{*}(\mathbf{n})\right)\right\} \prod_{(\mathbf{n}, k) \in E_{G}} f\left(\tau, L(\mathbf{n}), L\left(\mathbf{n}+\mathbf{e}_{i}\right)\right),
$$

where $z_{0}$ is the single site integral of the partition function for $\tau=0$ :

$$
\begin{aligned}
\left.Z\right|_{\tau=0} & \left.=\left(\int_{\mathrm{SU}(3)} \mathrm{d} W \exp \left\{\eta L+\bar{\eta} L^{*}\right)\right\}\right)^{N_{s}} \\
& :=\left(z_{0}\right)^{N_{s}} .
\end{aligned}
$$

In other words, if we denote by \langle\rangle$_{0}$ expectation values in the $\tau=0$ ensemble, the graph weight reads

$$
\varphi(G)=\left\langle\prod_{(\mathbf{n}, k) \in E_{G}} f\left(\tau, L(\mathbf{n}), L\left(\mathbf{n}+\mathbf{e}_{i}\right)\right)\right\rangle_{0}
$$

Combining the expansion of the product and the definition of the graph weights one obtains

$$
Z=z_{0}^{N_{s}}\left(1+\sum_{G \in \mathcal{G}\left(G_{\Lambda_{s}}\right)} \varphi(G)\right)
$$

It is quite cumbersome to deal with the set $\mathcal{G}\left(G_{\Lambda_{s}}\right)$. Note, however, that from equation (3.18) it is clear that $\varphi$ for a graph $G$ factorizes over its connected components, i. e. if $G_{1}, \ldots, G_{n}$ are the connected components of $G$ then:

$$
\varphi(G)=\varphi\left(\bigcup_{i=1}^{n} G_{i}\right)=\prod_{i=1}^{n} \varphi\left(G_{i}\right)
$$

Therefore, if we consider connected graphs to be polymers, define two connected graphs to be linked when their graph union is itself also a connected graph, denote by $\mathcal{G}_{c}(G)$ the subset of $\mathcal{G}(G)$, which contains only connected graphs, and define $\mathcal{D}_{n}\left(\mathcal{G}_{c}\left(G_{\Lambda_{s}}\right)\right)$ just like in section 2.2 the partition function reads

$$
\begin{aligned}
Z & =Z\left(\mathcal{G}_{c}\left(G_{\Lambda_{s}}\right)\right) \\
& =z_{0}^{N_{s}}\left(1+\sum_{n=1}^{\infty} \sum_{\left\{G_{1}, \ldots, G_{n}\right\} \in \mathcal{D}_{n}\left(\mathcal{G}_{c}\left(G_{\Lambda_{s}}\right)\right)} \varphi\left(G_{1}\right) \cdots \varphi\left(G_{n}\right)\right)
\end{aligned}
$$

Now the polymer expansion formula can be readily applied, leading to

$$
\log (Z)=N_{s} \log \left(z_{0}\right)+\sum_{n=1}^{\infty} \sum_{\left\{G_{1}, \ldots, G_{n}\right\} \in \mathcal{C}_{n}\left(\mathcal{G}_{c}\left(G_{\Lambda_{s}}\right)\right)} \sum_{\left(k_{1}, \ldots, k_{n}\right) \in\left(\mathbb{N}^{*}\right)^{n}} C_{\left(G_{1}, k_{1}\right), \ldots,\left(G_{n}, k_{n}\right)} \varphi\left(G_{1}\right)^{k_{1}} \cdots \varphi\left(G_{n}\right)^{k_{n}}
$$

As the union of the graphs in an element of $\mathcal{C}_{n}\left(\mathcal{G}_{c}\left(G_{\Lambda_{s}}\right)\right)$ is itself a connected subgraph of $G_{\Lambda_{s}}$ one can rewrite the logarithm to

$$
\log (Z)=N_{s} \log \left(z_{0}\right)+\sum_{G \in \mathcal{G}_{c}\left(G_{\Lambda_{s}}\right)} \xi(G),
$$


where $\xi(G)$ contains all contributions of linked clusters covering $G$ :

$$
\xi(G)=\sum_{n=1}^{\left|\mathcal{G}_{c}(G)\right|} \sum_{\left\{g_{1}, \ldots, g_{n}\right\} \in \mathcal{C}_{n}\left(\mathcal{G}_{c}(G)\right)} \sum_{\left(k_{1}, \ldots, k_{n}\right) \in\left(\mathbb{N}^{*}\right)^{n}} \delta\left(\bigcup_{i=1}^{n} g_{i}=G\right) C_{\left(g_{1}, k_{1}\right), \ldots,\left(g_{n}, k_{n}\right)} \varphi\left(g_{1}\right)^{k_{1}} \cdots \varphi\left(g_{n}\right)^{k_{n}} .
$$

In section 4.4 .3 we will explain how to fully determine $\xi$ without having to deal with the infinite sums in the above formula, for now we want to focus on another aspect however.

Inspecting equation (3.18) closer, one sees that the weight of a graph reduces to single site integrals at its vertices and these single site integrals are determined by the degrees of the vertices and which vertices are connected. This means that isomorphic graphs have the same weight and consequently $\xi$ evaluates to the same result for isomorphic graphs. To make use of that, we define a weak embedding of an undirected graph $g$ in another undirected graph $G$ as mappings $w_{V}: V_{g} \rightarrow V_{G}$ and $w_{E}: E_{g} \rightarrow E_{G}$ with the property that

- they are injective and

- $w_{V}\left(\sigma_{g}(e)\right)=\sigma_{G}\left(w_{E}(e)\right)$.

Note that $w$ is a graph isomorphism between $g$ and a subgraph of $G$. So, denoting by $\mathcal{W}(g ; G)$ the set of all weak embeddings of $g$ in $G$, equation (3.28) reduces to a summation over the quotient set $\mathcal{G}_{c}\left(G_{\Lambda_{s}}\right) / \sim$, where $\sim$ is the equivalence relation indicating isomorphic graphs:

$$
\log (Z)=N_{s} \log \left(z_{0}\right)+\sum_{G \in \mathcal{G}_{c}\left(G_{\Lambda_{s}}\right) / \sim} \frac{\left|\mathcal{W}\left(G ; G_{\Lambda_{s}}\right)\right|}{S(G)} \xi(G)
$$

The symmetry factor $S(G)$ of the graph has to be included because composing a weak embedding with a graph automorphism leads to the same subgraph of $G_{\Lambda_{s}}$. Due to the cyclic boundary conditions and since we only consider connected graphs, the size of $\left|\mathcal{W}\left(G ; G_{\Lambda_{s}}\right)\right|$ is proportional to $N_{s}$ if $G$ cannot loop the torus. Consequently, it makes sense to define the number of embeddings per site

$$
\left(G ; G_{\Lambda_{s}}\right)_{w}=\frac{\left|\mathcal{W}\left(G ; G_{\Lambda_{s}}\right)\right|}{N_{s}},
$$

which we will call the weak embedding number. The thermodynamic limit of this quantity is clearly well defined and defines the weak embedding number of a graph on $\mathbb{Z}^{3},\left(G ; \mathbb{Z}^{3}\right)_{w}$. Therefore, the free energy density in the thermodynamic limit reads

$$
\begin{aligned}
f & =-\lim _{N_{s} \rightarrow \infty} \frac{\log (Z)}{N_{s}} \\
& =-\log \left(z_{0}\right)-\sum_{G \in \mathcal{G}_{c, s}} \frac{\left(G ; \mathbb{Z}^{3}\right)_{w}}{S(G)} \xi(G),
\end{aligned}
$$

where $\mathcal{G}_{c, s}$ is the set of all connected simple graphs with more than one vertex and isomorphic graphs identified.

To count the weak embeddings per site of a graph $G$ in $G_{\Lambda_{s}}$ (for the case where $G_{\Lambda_{s}}$ is large enough such that that the graph to embed will not loop the torus), one can choose an arbitrary vertex and count the number of embeddings which map this vertex to $(0,0,0)$. A more efficient procedure to compute the weak embedding number is given in [123]

In general the main problem when computing the weak embedding number is the constraint that weak embeddings have to be injective, so no vertex can be placed at the same lattice site. Therefore, when iteratively placing vertices, one has to keep track of the vertices which have been 
placed so far, complicating the matter. The aforementioned constraint is removed in the case of the free graph expansion, simplifying matters and enabling so called renormalization schemes ${ }^{1}$. We will not further explore the weak graph expansion for the spin model here, because the free graph expansion can also be applied to it, as we will see in the next section.

\subsection{Free graph expansion of the spin model}

The weak graph expansion of the previous section was basically a direct application of the version of the polymer expansion, where the polymer moments are only used to indicate if the polymers are disjoint and the polymer weights contained the expectation values in the $\tau=0$ ensemble. When deriving the free graph expansion, the polymer moments will contain the expectation values. The application of the polymer expansion will then also lead to a weak graph expansion, which can, however, be rearranged to a free graph expansion. For introductions to the free graph expansions see $[124,125]$. It is the latter reference where the phrase 'free graph expansion' is suggested to be used instead of, as it is often done, 'linked cluster expansion', a term whose ambiguity we already mentioned in section 2.2. A well known application of the free graph expansion is to the $\phi^{4}$ theory [126], where it was used to prove its triviality [127-129]. Extensions of the work on the $\phi^{4}$ theory to higher orders, non-trivial vacuums and finite volume can be found in [130-132]. As mentioned before, the free graph expansion enables renormalization schemes, and we will apply the so-called 'vertex-renormalization'. It is possible to carry the renormalization procedure even further by an additional 'bond-renormalization'. An impressive example for the application of both renormalizations is [133], where the two-point Green's functions for a generalized 3-dimensional Ising model are obtained to 25 th order on the $b c c$ lattice and to $23 \mathrm{rd}$ order on the $s c$ lattice. What the renormalizations procedures have in common is that they reduce the number of graphs one has to consider, however at the price of increased algebraic complexity. To discuss these procedures in detail, we first must explain how to obtain the basic unrenormalized expansion. We previously outlined the free graph expansion of the spin model in [134], the following discussion includes some more details.

\subsubsection{Unrenormalized expansion}

The first difference in the derivation of the free graph expansion in comparison to the the previous section is that one directly expands the exponential containing the nearest neighbor interaction without turning the nearest neighbor sum into a product.

$$
\begin{aligned}
Z & =\int \mathrm{d}[W] \exp \left\{\sum_{\mathbf{n} \in \Lambda_{s}}\left(\tau \sum_{k=1}^{3}\left[L(\mathbf{n}) L^{*}\left(\mathbf{n}+\mathbf{e}_{k}\right)+L^{*}(\mathbf{n}) L\left(\mathbf{n}+\mathbf{e}_{k}\right)\right]+\eta L(\mathbf{n})+\bar{\eta} L^{*}(\mathbf{n})\right)\right\} \\
& =\int \mathrm{d}[W] \exp \left\{\tau \sum_{(\mathbf{n}, k) \in E_{G_{\Lambda_{s}}}}\left[L(\mathbf{n}) L^{*}\left(\mathbf{n}+\mathbf{e}_{k}\right)+L^{*}(\mathbf{n}) L\left(\mathbf{n}+\mathbf{e}_{k}\right)\right]\right\} \exp \left\{\sum_{\mathbf{n} \in \Lambda_{s}} \eta L(\mathbf{n})+\bar{\eta} L^{*}(\mathbf{n})\right\}
\end{aligned}
$$

\footnotetext{
${ }^{1}$ Which should not be confused with the renormalization procedure to remove ultraviolet divergences in quantum field theories.
} 


$$
\begin{aligned}
& =\sum_{n=0}^{\infty} \frac{\tau^{n}}{n !} \sum_{\left(\mathbf{n}_{1}, k_{1}\right), \ldots,\left(\mathbf{n}_{n}, k_{n}\right) \in E_{G_{\Lambda_{s}}}} \int \mathrm{d}[W]\left(\prod_{l=1}^{n}\left[L\left(\mathbf{n}_{l}\right) L^{*}\left(\mathbf{n}_{l}+\mathbf{e}_{k_{l}}\right)+L^{*}\left(\mathbf{n}_{l}\right) L\left(\mathbf{n}_{l}+\mathbf{e}_{k_{l}}\right)\right]\right. \\
& \left.\quad \times \exp \left\{\sum_{\mathbf{n} \in \Lambda_{s}} \eta L(\mathbf{n})+\bar{\eta} L^{*}(\mathbf{n})\right\}\right) \\
& =z_{0}^{N_{s}} \sum_{n=0}^{\infty} \frac{\tau^{n}}{n !} \sum_{\left(\mathbf{n}_{1}, k_{1}\right), \ldots,\left(\mathbf{n}_{n}, k_{n}\right) \in E_{G_{\Lambda_{s}}}}\left\langle\prod_{l=1}^{n}\left[L\left(\mathbf{n}_{l}\right) L^{*}\left(\mathbf{n}_{l}+\mathbf{e}_{k_{l}}\right)+L^{*}\left(\mathbf{n}_{l}\right) L\left(\mathbf{n}_{l}+\mathbf{e}_{k_{l}}\right)\right]\right\rangle_{0} .
\end{aligned}
$$

The expectation value can be rewritten by expanding the product inside it. Introducing the notation

$$
\begin{aligned}
& L_{1}=L, \\
& L_{2}=L^{*},
\end{aligned}
$$

the expanded version can be written as

$$
\sum_{\sigma_{1}, \ldots, \sigma_{n} \in S_{2}}\left\langle\prod_{l=1}^{n} L_{\sigma_{l}(1)}\left(\mathbf{n}_{l}\right) L_{\sigma_{l}(2)}\left(\mathbf{n}_{l}+\mathbf{e}_{k_{l}}\right)\right\rangle_{0}
$$

Therefore

$$
Z=z_{0}^{N_{s}} \sum_{n=0}^{\infty} \frac{\tau^{n}}{n !} \sum_{\left(\left(\mathbf{n}_{1}, k_{1}\right), \sigma_{1}\right), \ldots,\left(\left(\mathbf{n}_{n}, k_{n}\right), \sigma_{n}\right) \in E_{G_{\Lambda_{s}}} \times S_{2}}\left\langle\prod_{l=1}^{n} L_{\sigma_{l}(1)}\left(\mathbf{n}_{l}\right) L_{\sigma_{l}(2)}\left(\mathbf{n}_{l}+\mathbf{e}_{k_{l}}\right)\right\rangle_{0} .
$$

To apply the polymer expansion formula, we now declare the elements of $E_{G_{\Lambda_{s}}} \times S_{2}$ (which can be thought of as directed edges) to be the polymers. The weights are

$$
\varphi(((\mathbf{n}, k), \sigma))=\tau
$$

and the polymer moments are the expectation values of products of nearest neighbor pairs

$$
m\left(\left(\left(\mathbf{n}_{1}, k_{1}\right), \sigma_{1}\right), \ldots,\left(\left(\mathbf{n}_{n}, k_{n}\right), \sigma_{n}\right)\right)=\left\langle\prod_{l=1}^{n} L_{\sigma_{l}(1)}\left(\mathbf{n}_{l}\right) L_{\sigma_{l}(2)}\left(\mathbf{n}_{l}+\mathbf{e}_{k_{l}}\right)\right\rangle_{0} .
$$

Using equation (2.62) we obtain

$$
\begin{aligned}
\log (Z(P))= & N_{s} \log \left(z_{0}\right) \\
& +\sum_{n=1}^{\infty} \sum_{\left(\left(\mathbf{n}_{1}, k_{1}\right), \sigma_{1}\right), \ldots,\left(\left(\mathbf{n}_{n}, k_{n}\right), \sigma_{n}\right) \in E_{G_{\Lambda_{s}}} \times S_{2}} \frac{1}{n !} c\left(\left(\left(\mathbf{n}_{1}, k_{1}\right), \sigma_{1}\right), \ldots,\left(\left(\mathbf{n}_{n}, k_{n}\right), \sigma_{n}\right)\right) \tau^{n} .
\end{aligned}
$$

In this case, $m$ factorizes over edges which do not share a vertex. Therefore a necessary condition for $c\left(\left(\left(\mathbf{n}_{1}, k_{1}\right), \sigma_{1}\right), \ldots,\left(\left(\mathbf{n}_{n}, k_{n}\right), \sigma_{l}\right)\right)$ not to vanish is that the graph assembled from the edges in the arguments of $c$ is connected. Note that this graph is not necessarily simple, as the same edge can occur several times, which we will interpret as multiple edges connecting the same vertices. This suggests to write $\log (Z)$ again as an expansion in terms of connected graphs (which are directed multigraphs in this case), where the graph weights are given by $c$. In this way we are lead to a weak graph expansion again.

It is however possible to rearrange the expansion to eliminate the excluded volume restrictions of the weak graph expansion [135]. To this end, note that the factorization of $m$ is due to the 
factorization of \langle\rangle$_{0}$ over single sites. This factorization of \langle\rangle$_{0}$ is however not completely exploited in $m$ (and consequently $c$ ), since we are considering nearest neighbor pairs. If we consider \langle\rangle$_{0}$ as the moments with the polymers being the $L^{(*)}$ at the lattice sites, then these moments factorize over single lattice sites and correspondingly the associated cumulants, which we will denote by []$_{0}$, vanish when they contain $L^{(*)}$ from different lattice sites. Single site expectation values are easily obtained by differentiation of $z_{0}$ :

$$
\left\langle L(\mathbf{n})^{n} L^{*}(\mathbf{n})^{m}\right\rangle_{0}=\frac{1}{z_{0}} \frac{\partial^{n}}{\partial \eta^{n}} \frac{\partial^{n}}{\partial \bar{\eta}^{n}} z_{0}
$$

and consequently

$$
\left[L(\mathbf{n})^{n} L^{*}(\mathbf{n})^{m}\right]_{0}=\frac{\partial^{n}}{\partial \eta^{n}} \frac{\partial^{n}}{\partial \bar{\eta}^{n}} \log \left(z_{0}\right) .
$$

Note that the result is independent of $\mathbf{n}$, so whenever it is not relevant, we will denote the $L^{(*)}$ without arguments. What happens now, if we replace the "edge cumulants" $c$ in equation (3.44) by the $\tau=0$ single site expectation value cumulants [] , which we will call, following [124], bare semi-invariants ${ }^{2}$ ?

Consider, for example, $n=2$, with $\mathbf{n}_{2}=\mathbf{n}_{1}+\mathbf{e}_{k_{1}}$ and $\sigma_{1}=$ id and $\sigma_{2}=(1,2)$, where we used cycle notation for $\sigma_{2}$. We also conveniently define $\mathbf{n}_{3}=\mathbf{n}_{2}+\mathbf{e}_{k_{2}}, e_{1}=\left(\left(\mathbf{n}_{1}, k_{1}\right), \sigma_{1}\right)$ and $e_{2}=\left(\left(\mathbf{n}_{2}, k_{2}\right), \sigma_{2}\right)$. Then

$$
\begin{aligned}
c\left(e_{1}, e_{2}\right)= & m\left(e_{1}, e_{2}\right)-m\left(e_{1}\right) m\left(e_{2}\right) \\
= & \left\langle L\left(\mathbf{n}_{1}\right) L^{*}\left(\mathbf{n}_{2}\right) L^{*}\left(\mathbf{n}_{2}\right) L\left(\mathbf{n}_{3}\right)\right\rangle_{0} \\
& -\left\langle L\left(\mathbf{n}_{1}\right) L^{*}\left(\mathbf{n}_{2}\right)\right\rangle_{0}\left\langle L^{*}\left(\mathbf{n}_{2}\right) L\left(\mathbf{n}_{3}\right)\right\rangle_{0} \\
= & \left\langle L\left(\mathbf{n}_{1}\right)\right\rangle_{0}\left\langle L^{*}\left(\mathbf{n}_{2}\right)^{2}\right\rangle_{0}\left\langle L\left(\mathbf{n}_{3}\right)\right\rangle_{0} \\
& -\left\langle L\left(\mathbf{n}_{1}\right)\right\rangle_{0}\left\langle L^{*}\left(\mathbf{n}_{2}\right)\right\rangle_{0}^{2}\left\langle L\left(\mathbf{n}_{3}\right)\right\rangle_{0} \\
= & {\left[L\left(\mathbf{n}_{1}\right)\right]_{0}\left[L^{*}\left(\mathbf{n}_{2}\right)^{2}\right]_{0}\left[L\left(\mathbf{n}_{1}\right)\right]_{0} }
\end{aligned}
$$

If we now also consider the case where $e_{2}=\left(\left(\mathbf{n}_{1}, k_{1}\right), \sigma_{2}\right)$ we see that

$$
\begin{aligned}
c\left(e_{1}, e_{2}\right)= & {\left[L\left(\mathbf{n}_{1}\right) L^{*}\left(\mathbf{n}_{1}\right)\right]_{0}\left[L\left(\mathbf{n}_{2}\right) L^{*}\left(\mathbf{n}_{2}\right)\right]_{0} } \\
& +\left[L\left(\mathbf{n}_{1}\right) L^{*}\left(\mathbf{n}_{1}\right)\right]_{0}\left[L\left(\mathbf{n}_{2}\right)\right]_{0}\left[L^{*}\left(\mathbf{n}_{2}\right)\right]_{0}+\left[L\left(\mathbf{n}_{1}\right)\right]_{0}\left[L^{*}\left(\mathbf{n}_{1}\right)\right]_{0}\left[L\left(\mathbf{n}_{2}\right) L^{*}\left(\mathbf{n}_{2}\right)\right]_{0} .
\end{aligned}
$$

These equations can be represented graphically:

$$
\begin{aligned}
& c(\bullet \bullet \bullet \bullet)=[L]_{0} \stackrel{\bullet}{\left[\left(L^{*}\right)^{2}\right]_{0}} \underset{\bullet}{\leftrightarrow}[L]_{0},
\end{aligned}
$$

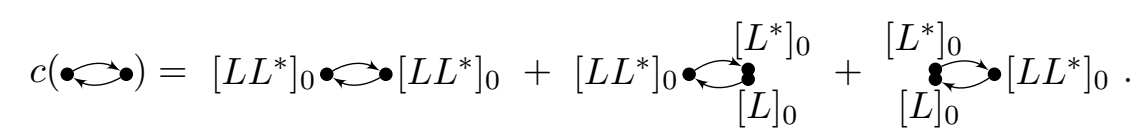

This suggests that if we replace the graph weight of a graph given by $c$ by an expression in terms of []$_{0}$, this corresponds to a sum over new weights of connected graphs that can be embedded freely in the original graph. The new weights here are defined to be

$$
W^{0}=\prod_{v \in V(G)}\left[L^{n_{\text {out }}(v)}\left(L^{*}(v)\right)^{n_{\text {in }}}\right]_{0},
$$

\footnotetext{
${ }^{2}$ The authors of [125] call these quantities bare vertex functions and in [126] they are named cumulant moments.
} 
and by freely we mean that when embedding $g$ in the graph $G$, distinct vertices of $g$ can occupy the same vertex of $G$, as it is the case for the last two terms in the last equation.

We infer the general rule here from two suggestive examples and refer to [135] for a more detailed investigation.

To formulate the consequences of the replacements for equation (3.44) we define free embeddings of a directed graph $g$ in an undirected graph $G$ to be two mappings $f_{V}: V_{g} \rightarrow V_{G}$ and $f_{E}: E_{g} \rightarrow E_{G}$ with the property that

$$
f_{V}\left(i_{g}(e), t_{g}(e)\right)=\sigma_{G}\left(f_{E}(e)\right) \text { for alle } \in E_{g} .
$$

We stress that in contrast to weak embeddings, $f_{V}$ and $f_{E}$ do not have to be injective. In other words the only thing one has to take care of when embedding a graph freely is that vertices connected by an edge are placed on vertices which are also connected by an edge. The embeddings per site $\left(G ; G_{\Lambda_{s}}\right)_{f}$ can be defined just like in the weak case and equation (3.44) can be rewritten to

$$
\log (Z)=N_{s} \log \left(z_{0}\right)+\sum_{G \in \mathcal{G}_{c, d}} \tau^{|E(G)|} \frac{\left(G ; G_{\Lambda_{s}}\right)_{f}}{S(G)} W^{0}(G),
$$

where $\mathcal{G}_{c, d}$ is the set of all connected and directed (multi-)graphs, up to isomorphisms, with at least one edge. Note that associating the graph with only one vertex and no edges with $\log \left(z_{0}\right)$ is consistent with equation (3.46). Of course one cannot actually sum all graphs, and one truncates the expansion at a certain order in $\tau$. Therefore it is useful to introduce the notation $\mathcal{G}_{c, d}^{(i)}$ for the subset of $\mathcal{G}_{c, d}$ which contains graphs with $i$ edges. We finally obtain for the free energy density in the thermodynamic limit

$$
\begin{aligned}
-f & =\lim _{N_{s} \rightarrow \infty} \frac{\log (Z)}{N_{s}} \\
& =\sum_{i=0}^{\infty} \tau^{i} \sum_{\mathcal{G}_{c, d}^{(i)}} \frac{\left(G, \mathbb{Z}^{3}\right) W^{0}(G)}{S(G)}
\end{aligned}
$$

To compute the free energy density using this formula to a certain order, one has to list all graphs relevant to this order and compute their symmetry numbers, free embedding numbers and weights. Of course, to go to higher orders, it is necessary to automate this task using a computer. For the generation of the graphs, it is important to avoid generating isomorphic graphs or to remove them as early as possible. This is best be done in a representation of the graphs which allows for fast isomorphism checking. To this end one introduces the concept of a canonical labeling of a graph, which is an enumeration of the vertices of a graph such that two isomorphic graphs have the same adjacency matrix. This is a well known problem in mathematics and specialized software for this problem exists [136, 137], in our investigations we used McKay's nauty. As a useful side effects, the computation of canonical labelings can also give the automorphism group and its order (i.e. the symmetry number) of a graph. For the free embedding number, a procedure to compute it is explained in [126], which was implemented during this thesis in Mathematica.

Finally the weights necessitate the computation of the bare semi-invariants according to equation (3.46). This is especially easy when $\log \left(z_{0}\right)$ is known as an expansion in terms of $\eta$ and $\bar{\eta}$. In order to obtain this expansion, consider

$$
z_{0}(\alpha, \eta, \bar{\eta})=\int_{\mathrm{SU}(3)} \mathrm{d} W \exp \left[\alpha\left(\eta L+\bar{\eta} L^{*}\right)\right]
$$

The series expansion of $\log \left(z_{0}(\alpha, \eta, \bar{\eta})\right)$ to $\mathcal{O}\left(\alpha^{n}\right)$ corresponds to an expansion to $\mathcal{O}\left(\eta^{n_{1}} \eta^{n_{2}}\right)$ with $n_{1}+n_{2}=n$. To compute this expansion, we need to know the derivatives of $\log \left(z_{0}(\alpha, \eta, \bar{\eta})\right)$. Faà 
di Bruno's formula states that the n'th derivative of the composition $f \circ g$ reads [138]

$$
\frac{\mathrm{d}^{n}}{\mathrm{~d} x^{n}} f(g(x))=\sum_{k=1}^{n} f^{(k)}(g(k)) B_{n, k}\left(\frac{\mathrm{d} g}{\mathrm{~d} x}, \ldots, \frac{\mathrm{d}^{n-k+1} g}{\mathrm{~d} x^{n-k+1}}\right) .
$$

where $B_{n, k}$ denotes the partial Bell polynomial

$$
B_{n, k}=\sum_{j \in \mathbb{N}^{n-k+1}} \delta\left(\sum_{i=1}^{n-k+1} j_{i}=k\right) \delta\left(\sum_{i=1}^{n-k+1} i j_{i}=n\right) n ! \prod_{i=1}^{n-k+1} \frac{1}{j_{i}}\left(\frac{x_{i}}{i !}\right)^{j_{i}}
$$

The derivatives of $\log (1+x)$ are of course well known and therefore

$$
\log \left(z_{0}(\alpha, \eta, \bar{\eta})\right)=\sum_{n=1}^{\infty} \sum_{k=1}^{n}(-1)^{k+1} \frac{(k-1) !}{n !} B_{n, k}\left(\left.\frac{\partial z}{\partial a}\right|_{\alpha=0}, \ldots,\left.\frac{\partial^{n-k+1} z}{\partial \alpha^{n-k+1}}\right|_{\alpha=0}\right) \alpha^{n} .
$$

Using the binomial theorem the derivatives of $z_{0}$ evaluate to

$$
\begin{aligned}
\left.\frac{\partial^{p} z}{\partial \alpha^{p}}\right|_{\alpha=0} & =\left.\frac{\partial^{p}}{\partial \alpha^{p}}\right|_{\alpha=0} \int_{\mathrm{SU}(3)} \mathrm{d} W \exp \left[\alpha\left(\eta L+\bar{\eta} L^{*}\right)\right] \\
& =\int_{\mathrm{SU}(3)} \mathrm{d} W\left(\eta L+\bar{\eta} L^{*}\right)^{p} \\
& =\sum_{q=0}^{p}\left(\begin{array}{l}
p \\
q
\end{array}\right) I_{q, p-q} \eta^{q} \bar{\eta}^{p-q}
\end{aligned}
$$

where we defined the SU(3)-integral

$$
I_{q, p-q}=\int_{\mathrm{SU}(3)} \mathrm{d} W L^{q} L^{*(p-q)} .
$$

An explicit formula for the evaluation of this integral is given in [67].

We now have all ingredients to obtain arbitrary orders in the series expansion of the free energy density of the spin model around $\tau=0$, the only limit being available computing time. One of the main bottlenecks for this computation is, that the number of graphs one has to consider when increasing the orders grows very fast. This problem can be alleviated using the renormalization procedures mentioned before.

\subsubsection{Vertex-renormalized expansion}

The observation underlying the vertex renormalization is that when a graph has an articulation point, then the free embedding number of that graph is the product of the free embedding numbers of the two graphs that are obtained by splitting the original graph at its articulation point. Graphically (clearly the direction of the edges does not matter for the free embeddings):

$$
\left.\bigcirc_{i} ; G\right)_{f} \times(j G)_{f}
$$

Of course for $G=\mathbb{Z}^{n}$ the last embedding number vanishes but this equation is only for illustrative purpose. Using this, an expansion in 1-irreducible graphs, i. e. graphs with no articulation points, can be assembled to give the full expansion. The details on why this procedure works are given in [124], and to see how it is related to a Legendre transformation, see [139]. 
We just show how the application to the spin-model works. First we collect all connected graphs that can occur after splitting a graph at an articulation point in the so-called self fields. The vertex that was the articulation point of the original graph gets a special treatment and is therefore considered to be and external vertex. Consequently the connected graphs that occur in the self fields are one insertions and we define $\mathcal{I}_{n, m}^{(i)}$ to be the set of all one-insertions with $i$ edges and where the external vertex has out-degree $n$ and in-degree $m$. The contribution of the vertex that was the articulation point to the weight of a graph has to be considered separately, as it depends on all of the graphs that occur after splitting a graph at an articulation point. Due to this fact, we generalize the weight of a graph from equation (3.54) to graphs with an external vertex to be

$$
W^{0}=\prod_{v \in I_{G}}\left[L^{n_{\text {out }}(v)}\left(L^{*}(v)\right)^{n_{\text {in }}}\right]_{0} .
$$

Eventually, we define the self-fields

$$
G_{n, m}=\sum_{i=n+m}^{\infty} \tau^{i} \sum_{G \in \mathcal{I}_{(n, m)}^{(i)}} \frac{\left(G, \mathbb{Z}^{3}\right)_{f} W^{0}(G)}{S(G)}
$$

When joining one-insertions at their external vertex one obtains a new graph, this graph has an articulation point at the vertex where the one-insertions were joined. The contribution of this articulation point to the weights of all graphs which are obtained by joining one-insertions in this way is contained in the renormalized semi-invariants, which read

$$
\begin{aligned}
{\left[L^{n}\left(L^{*}\right)^{m}\right]_{\mathrm{ren}}=} & {\left[L^{n}\left(L^{*}\right)^{m}\right]_{0} } \\
& +\sum_{p=1}^{\infty} \frac{1}{p !} \sum_{\left(l_{1}, k_{1}\right), \ldots,\left(l_{k}, k_{p}\right) \in \mathbb{N}^{2} \backslash(0,0)}\left(\prod_{j=1}^{p} G_{l_{j}, k_{j}}\right)\left[L^{n+l_{1}+\cdots+l_{p}}\left(L^{*}\right)^{m+k_{1}+\cdots+k_{p}}\right]_{0} \\
= & \exp \left(\sum_{(l, k) \in \mathbb{N}^{2} \backslash(0,0)} G_{l, k}\left(\frac{\partial}{\partial \eta}\right)^{l}\left(\frac{\partial}{\partial \bar{\eta}}\right)^{k}\right) \log \left(z_{0}\right)
\end{aligned}
$$

One can then show that the renormalized semi-invariants correspond to the single site cumulant expectation values for the full theory (i.e. for $\tau \neq 0$ ) so one could expect that $[1]_{\text {ren }}=-f$. This, however, is not true. Instead, one at first has to define renormalized graph weights $W(G)$

$$
W(G)=\prod_{v \in I(G)}\left[L^{n_{\text {out }}(v)}\left(L^{*}(v)\right)^{n_{\text {in }}}\right]_{\mathrm{ren}} .
$$

Then, the so-called $\Phi$-functional can be obtained via

$$
\Phi=\sum_{i=1}^{\infty} \tau^{i} \sum_{G \in \mathcal{G}_{(1)}^{i}} \frac{\left(G ; \mathbb{Z}^{3}\right)_{f} W(G)}{S(G)}
$$

where $\mathcal{G}_{1}^{(i)}$ is the subset of $\mathcal{G}_{c, d}^{(i)}$ that contains only 1-irreducible graphs. Finally, the free energy density in the renormalized scheme reads

$$
-f=[1]_{\mathrm{ren}}+\Phi-\sum_{l, k \in \mathbb{N}^{*}} G_{l, k} M_{l, k}
$$

While the sets $\mathcal{G}_{1}^{(i)}$, that occur in $\Phi$, are much smaller than the sets $\mathcal{G}_{c, d}$, to obtain the free en- 
ergy density one also has to compute the self fields, as we have seen, and those contain a sum over all 1-insertion, including those which are not 1-irreducible, according to equation (3.69). Therefore, for practical calculations, no reduction in graph complexity has been achieved at this point. One can show however, that if one replaces the bare semi-invariants in the weights occurring in equation (3.69) by their renormalized counterparts, the sums can be restricted to 1-irreducible 1-insertions $\mathcal{I}_{1,(n, m)}^{(i)}$

$$
G_{n, m}=\sum_{i=n+m}^{\infty} \tau^{i} \sum_{G \in \mathcal{I}_{1,(n, m)}^{(i)}} \frac{\left(G, \mathbb{Z}^{3}\right)_{f} W^{0}(G)}{S(G)} .
$$

While now all relevant equations to obtain the free energy density only contain sums over 1irreducible graphs, equation (3.75) and equation (3.70) are coupled equations and this coupling needs to be resolved in calculations. To this end, an iterative strategy is used that determines the self fields and renormalized semi-invariants up to the ordered that is necessary for the expansion. If the aim is to determine the free energy density to $\mathcal{O}\left(\tau^{n_{\max }}\right)$ then one has to obtain the renormalized semi-invariant $\left[L^{n}\left(L^{*}\right)^{m}\right]_{\text {ren }}$ to order $n_{\max }-n-m$. Since the lowest order contribution of the selffield $G_{n, m}$ is $\mathcal{O}\left(\tau^{n+m}\right)$ all self-fields $G_{n, m}$ with $n+m \leq n_{\max }$ have to be calculated to $n_{\max }$. For the same reason, the leading order in equation (3.70) simply reads

$$
\left[L^{n}\left(L^{*}\right)^{m}\right]_{\mathrm{ren}}=\left[L^{n}\left(L^{*}\right)^{m}\right]_{0}+\mathcal{O}(\tau) .
$$

This can be used to determine $G_{1,0}$ and $G_{0,1}$ to $\mathcal{O}(\tau)$ using equation (3.75). This result can in turn be used to attain the renormalized semi-invariants to order 1 in $\tau$. Generically, once the renormalized semi-invariants $\left[L^{n}\left(L^{*}\right)^{m}\right]$ are available to order $p$ with $(n+m) \leq p$, one can use equation (3.75) to get the self fields $G_{n, m}$ to order $p+1$, which then can be used to determine the renormalized semi-invariant to order $p+1$. This can be repeated until the desired order in $\tau$ is achieved. When implementing this procedure on the computer, one can use the previously mentioned tools for graph canonicalization. An additional problem arises then as these tools cannot directly compute the canonical labeling for a directed multigraph with external vertices. We circumvent this problem in the following way: a canonical labeling is a special enumeration of the vertices. To distinguish an external vertex in the adjacency matrix arising from an enumeration we add a loop to the vertex. We then consider all canonical labelings of the underlying simple graph and they can be computed using the above mentioned tools. Then a canonical labeling is obtained by selecting the enumeration which minimizes the adjacency matrix.

To illustrate the general equations, we calculate the free energy density both in the unrenormalized and renormalized scheme.

\subsubsection{Example: Deriving the free energy density to $\mathcal{O}\left(\tau^{2}\right)$}

To compute the free energy density in the unrenormalized scheme, we need to list all connected and directed graphs with no external vertices and up to two edges, see table 3.1. In the table, we also included the free embedding numbers, graph weights and symmetry numbers, as demanded by equation (3.58). This equation can now be evaluated to $\mathcal{O}\left(\tau^{2}\right)$ using the table resulting in

$$
\begin{gathered}
-f=\log \left(z_{0}\right)+\tau\left(6[L]_{0}\left[L^{*}\right]_{0}\right) \\
+\tau^{2}\left(3\left[L^{2}\right]_{0}\left[\left(L^{*}\right)^{2}\right]_{0}+3\left[L\left(L^{*}\right)\right]_{0}^{2}+36[L]_{0}\left[L L^{*}\right]_{0}\left[L^{*}\right]_{0}+\right. \\
\left.18[L]_{0}^{2}\left[\left(L^{*}\right)^{2}\right]_{0}+18\left[L^{*}\right]_{0}^{2}\left[L^{2}\right]_{0}\right) .
\end{gathered}
$$




\begin{tabular}{cccc} 
Graph & $S$ & $M$ & $W^{0}$ \\
\hline & 1 & 1 & $\log \left(z_{0}\right)$ \\
& 1 & $2 d$ & {$[L]_{0}\left[L^{*}\right]_{0}$} \\
& 2 & $2 d$ & {$\left[L^{2}\right]_{0}\left[\left(L^{*}\right)^{2}\right]_{0}$} \\
& 2 & $2 d$ & {$\left[L\left(L^{*}\right)\right]_{0}^{2}$} \\
& 2 & $(2 d)^{2}$ & {$[L]_{0}\left[L L^{*}\right]_{0}\left[L^{*}\right]_{0}$} \\
& & $(2 d)^{2}$ & {$[L]_{0}^{2}\left[\left(L^{*}\right)^{2}\right]_{0}$}
\end{tabular}

Table 3.1: Taken from [134]. Graphs considered for the free energy density up to $\mathcal{O}\left(\tau^{2}\right)$ with their symmetry numbers $S$, embedding numbers $M$ on a $d$-dimensional square lattice and weights.

\begin{tabular}{cccc} 
Graph & $S$ & $M$ & $W$ \\
\hline$\dot{i}$ & 1 & $2 d$ & {$\left[L^{*}\right]_{\mathrm{ren}}$} \\
$\vdots$ & 1 & $2 d$ & {$[L]_{\mathrm{ren}}$} \\
0 & 2 & $2 d$ & {$\left[\left(L^{*}\right)^{2}\right]_{\mathrm{ren}}$} \\
0 & 2 & $2 d$ & {$\left[L^{2}\right]_{\mathrm{ren}}$} \\
0 & 1 & $2 d$ & {$[L]_{\mathrm{ren}}\left[L^{*}\right]_{\mathrm{ren}}$}
\end{tabular}

Table 3.2: Taken from [134]. Graphs considered for the self fields up to $\mathcal{O}\left(\tau^{2}\right)$ in the vertexrenormalized scheme. External vertices are depicted as unfilled circles.

The bare semi-invariants can then be determined to arbitrary precision using equation (3.46) and equation (3.62). This is best left to a computer and doing this calculation here would not make this example any more elucidating.

Next, we want to see how the same calculation works in the renormalized scheme. The first step is to determine the renormalized semi-invariants and the self-fields to $\mathcal{O}\left(\tau^{2}\right)$. We already know that the self-field $G_{n, m}$ vanishes for orders below $n+m$, so to $\mathcal{O}\left(\tau^{2}\right)$ equation (3.70) reads

$$
\begin{aligned}
{\left[L^{n}\left(L^{*}\right)^{m}\right]_{\mathrm{ren}}=} & {\left[L^{n}\left(L^{*}\right)^{m}\right]_{0} } \\
& +G_{0,1}\left[L^{n}\left(L^{*}\right)^{m+1}\right]_{0}+G_{1,0}\left[L^{n+1}\left(L^{*}\right)^{m}\right]_{0} \\
& +G_{0,2}\left[L^{n}\left(L^{*}\right)^{m+2}\right]_{0}+G_{2,0}\left[L^{n+2}\left(L^{*}\right)^{m}\right]_{0} \\
& +\left(G_{1,1}+G_{0,1} G_{1,0}\right)\left[L^{n+1}\left(L^{*}\right)^{m+1}\right]_{0} \\
& +\frac{1}{2} G_{0,1}^{2}\left[L^{n}\left(L^{*}\right)^{m+2}\right]_{0}+\frac{1}{2} G_{1,0}^{2}\left[L^{n+2}\left(L^{*}\right)^{m}\right]_{0} \\
& +\mathcal{O}\left(\tau^{3}\right) .
\end{aligned}
$$

Table 3.2 lists all 1-irreducible 1-insertions with less than three edges. Using equation (3.75) therefore gives

$$
G_{1,0}=6 \tau\left[L^{*}\right]_{\mathrm{ren}},
$$




$$
\begin{aligned}
G_{1,1} & =6 \tau^{2}\left[L L^{*}\right]_{\mathrm{ren}}+\mathcal{O}\left(\tau^{3}\right), \\
G_{2,0} & =3 \tau^{2}\left[\left(L^{*}\right)^{2}\right]_{\mathrm{ren}}+\mathcal{O}\left(\tau^{3}\right) .
\end{aligned}
$$

We note that $\mathcal{I}_{1,(1,0)}^{(i)}=\mathcal{I}_{(0,1)}^{(i)}=\emptyset$ for $i>1$ and therefore equation (3.79) is exact. Furthermore one can get $G_{m, n}$ from $G_{n, m}$ by making the exchange $\left[L^{n}\left(L^{*}\right)^{m}\right] \leftrightarrow\left[L^{m}\left(L^{*}\right)^{n}\right]$, which is equivalent to $\eta \leftrightarrow \bar{\eta}$. Therefore, it is enough to list only the self fields $G_{n, m}$ for $n \geq m$, as we did. Next, we have to use the iterative procedure explained in the previous section to decouple equation (3.78) from equations (3.79) to (3.81).

We know that to leading order $\left[L^{n}\left(L^{*}\right)^{m}\right]_{\mathrm{ren}}=\left[L^{n}\left(L^{*}\right)^{m}\right]_{0}$ and therefore equation (3.79) reads to leading order

$$
G_{1,0}=6 \tau\left[L^{*}\right]_{\mathrm{ren}}+\mathcal{O}\left(\tau^{2}\right)
$$

which implies

$$
G_{0,1}=6 \tau[L]_{\mathrm{ren}}+\mathcal{O}\left(\tau^{2}\right) .
$$

Inserting this into equation (3.78) we get to first order in $\tau$

$$
\begin{aligned}
{[1]_{\mathrm{ren}} } & =\log \left(z_{0}\right)+12 \tau\left[L^{*}\right]_{0}[L]_{0}+\mathcal{O}\left(\tau^{2}\right), \\
{[L]_{\mathrm{ren}} } & =[L]_{0}+6 \tau\left[L^{*}\right]_{0}\left[L^{2}\right]_{0}+6 \tau[L]_{0}\left[L L^{*}\right]_{0}+\mathcal{O}\left(\tau^{2}\right) .
\end{aligned}
$$

Using this we can determine the next order in the self-fields with the help of equations (3.79) to $(3.81)$ :

$$
\begin{aligned}
& G_{1,0}=6 \tau\left[L^{*}\right]_{0}+36 \tau^{2}\left[L^{*}\right]_{0}\left[L L^{*}\right]_{0}+36 \tau^{2}[L]_{0}\left[\left(L^{*}\right)^{2}\right]_{0}+\mathcal{O}\left(\tau^{3}\right), \\
& G_{1,1}=6 \tau^{2}\left[L L^{*}\right]_{0}+\mathcal{O}\left(\tau^{3}\right), \\
& G_{2,0}=3 \tau^{2}\left[\left(L^{*}\right)^{2}\right]_{0}+\mathcal{O}\left(\tau^{3}\right) .
\end{aligned}
$$

Of the renormalized semi-invariants, we only need $[1]_{\text {ren }}$ to $\mathcal{O}\left(\tau^{2}\right)$. Using equation (3.78) once more one gets (this equation is wrong in [134])

$$
\begin{aligned}
{[1]_{\mathrm{ren}}=} & \log \left(z_{0}\right)+12 \tau\left[L^{*}\right]_{0}[L]_{0} \\
& +6 \tau^{2}\left(\left[L^{2}\right]_{0}\left[\left(L^{*}\right)^{2}\right]_{0}+\left[L L^{*}\right]_{0}^{2}+9[L]_{0}^{2}\left[\left(L^{*}\right)^{2}\right]_{0}+9\left[L^{2}\right]_{0}\left[L^{*}\right]_{0}^{2}+18[L]_{0}\left[L^{*}\right]_{0}\left[L L^{*}\right]_{0}^{2}\right) .
\end{aligned}
$$

In the next step we determine the free energy density in terms of the renormalized semi-invariants and the self fields. To this end, we use equation (3.73) to obtain the $\Phi$-functional. equation (3.73) contains a sum over connected 1-irreducible graphs without external vertices. Since we go to $\mathcal{O}\left(\tau^{2}\right)$, we only need $\mathcal{G}_{1}^{(1)}$ and $\mathcal{G}_{1}^{(2)}$. The graphs in these sets are all contained in table 3.1. More precisely, graph 2 is the only member of $\mathcal{G}_{1}^{(1)}$ and graphs 3 and 4 constitute $\mathcal{G}_{1}^{(2)}$. When using these graphs for the $\Phi$-functional, one has to, of course, replace their weights by the renormalized version and equation (3.73) gives

$$
\Phi=6 \tau M_{0,1} M_{1,0}+3 \tau^{2}\left(M_{0,2} M_{2,0}+M_{1,1}^{2}\right) .
$$

Inserting this into equation (3.74), the free energy density reads

$$
\begin{aligned}
-f= & {[1]_{\mathrm{ren}}+6 \tau M_{0,1} M_{1,0}+3 \tau^{2}\left(M_{0,2} M_{2,0}+M_{1,1}^{2}\right) } \\
& -\left(G_{1,0}[L]_{\mathrm{ren}}+G_{0,1}\left[L^{*}\right]_{\mathrm{ren}}+G_{1,1}\left[L L^{*}\right]_{\mathrm{ren}}+G_{2,0}\left[L^{2}\right]_{\mathrm{ren}}+G_{0,2}\left[\left(L^{*}\right)^{2}\right]_{\mathrm{ren}}\right) .
\end{aligned}
$$

All that is left to do is to insert the expressions for the renormalized semi-invariants and the selffields we just derived into the last equation to obtain exactly the same result as equation (3.77). 
As one can see, the reduced number of graphs one has to consider leads to a greater algebraic complexity. For this example it might even seem that the renormalization procedure leads to more work to achieve the same order. We remark however that for higher orders the reduction in the number of graphs is much more significant. For example, there are 322737 undirected connected graphs with 13 edges, but only 3518 of those are 1-irreducible and in addition there are 24530 undirected 1-irreducible 1-insertions with 13 edges. Therefore, the number of graphs one has to consider in the renormalized scheme in this case is one order of magnitude smaller than the number of graphs for the unrenormalized scheme.

\subsection{Results from the series expansion}

Using the vertex-renormalized scheme we derived the the free energy density in the thermodynamic limit up to $\mathcal{O}\left(\tau^{14}\right)$ and to keep the size of the expressions under control, we also expanded all expressions containing the bare semi-invariants up to $\mathcal{O}\left(\kappa^{60}\right)$. To get an idea of the necessary compute, see [134], where we also summarized all necessary steps for the computation of the free energy density in the renormalized scheme. In the same paper, the obtained series was analyzed using different approaches. As the main contribution of the author of this thesis was the series expansion itself, we will only summarize the results of the analysis and refer to [134] for details.

Having obtained the free energy density, all other thermodynamic quantities can be derived from it. Monte-Carlo simulations cannot evaluate the free energy density directly, so to compare the series results to simulations we also consider the combination of first derivatives

$$
\Delta S=-\frac{\partial f}{\partial t}-\frac{\partial f}{\partial \eta}
$$

which in QCD is called interaction measure, as it is connected to the trace of the energy momentum tensor. For the investigation of phase transitions, we use connected correlation functions summed over the lattice

$$
\begin{aligned}
\chi & =-\frac{\partial f}{\partial \eta}-\frac{\partial f}{\partial \bar{\eta}}-\frac{\partial^{2} f}{\partial \eta \partial \bar{\eta}} \\
C & =-\frac{\partial^{2} f}{\partial \tau^{2}}
\end{aligned}
$$

which correspond to the magnetic susceptibility and the specific heat in the spin model analogy.

In figure 3.2, we show the three highest order of the series for the interaction measure. The figure shows good convergence of the series up to the neighborhood of the transition to the phase where the (approximate) center symmetry is spontaneously broken. Likewise, for $\mu=0$ good agreement with Monte-Carlo data is found up to the same region. Furthermore, we observe sensible results for $\mu \neq 0$, where standard Mont-Carlo simulations are not possible. The agreement with Monte-Carlos data can be extended up to the phase transition by employing Padé approximants with respect to the expansion parameter $\tau$. Padé approximants are rational functions whose Taylor expansions agree with the original series, for an introduction we refer to [140, 141]. Being rational functions, they can have singularities and are consequently better suited for the description of the behavior close to a phase transition (clearly they are best suited for the description of second-order behavior).

To study the location and order of the phase transition, we also employed Padé approximants, as well as the ratio test, in order to determine the radius of convergence of the series. As a reference we again point to $[140,141]$. In this case, it is more useful to use the Padé approximants for the logarithmic derivative of the observable (called DLog Padé), as this quantity has a simple pole in case of a second-order transition and can therefore be faithfully reproduced by Padé approximants. Furthermore, the residue of the Padé approximant at the singularity gives an estimate for the 

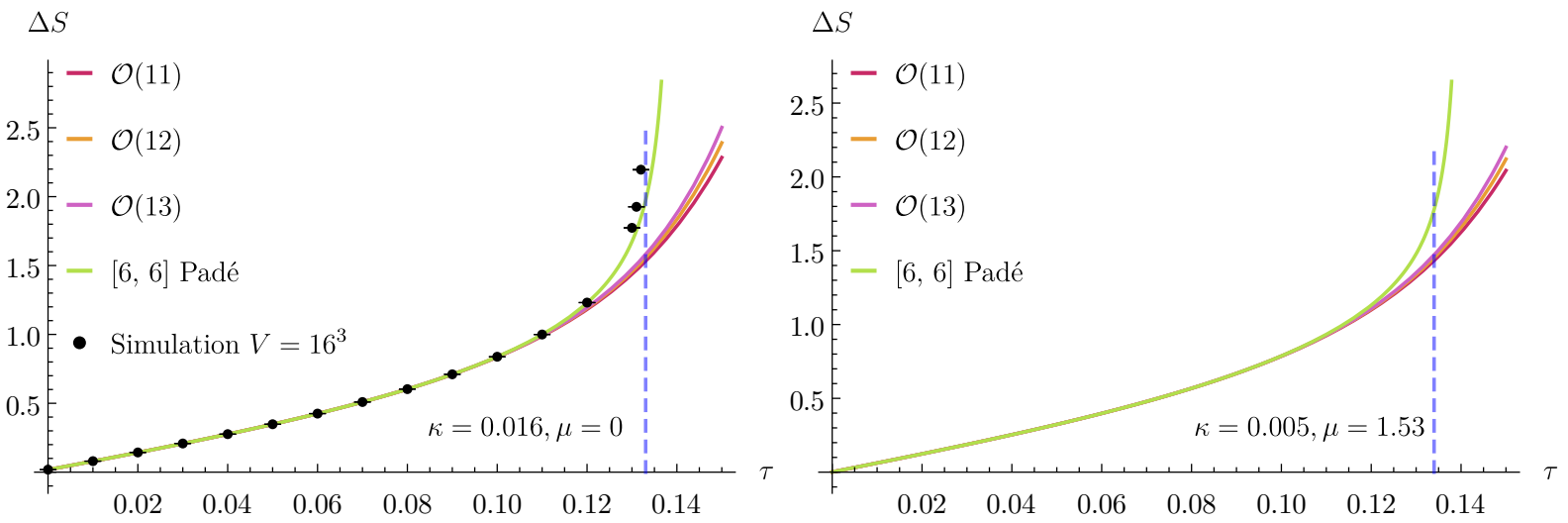

Figure 3.2: Taken from [134]. The interaction measure from the three highest order of the expansion. For vanishing chemical potential (left), comparisons to Monte-Carlo data can be made. We can also adjust the chemical potential to large values (right), where standard Monte-Carlo suffers from the sign problem. In both cases, we indicated the critical $\tau$ based on simulations of the dual flux representation in [68].
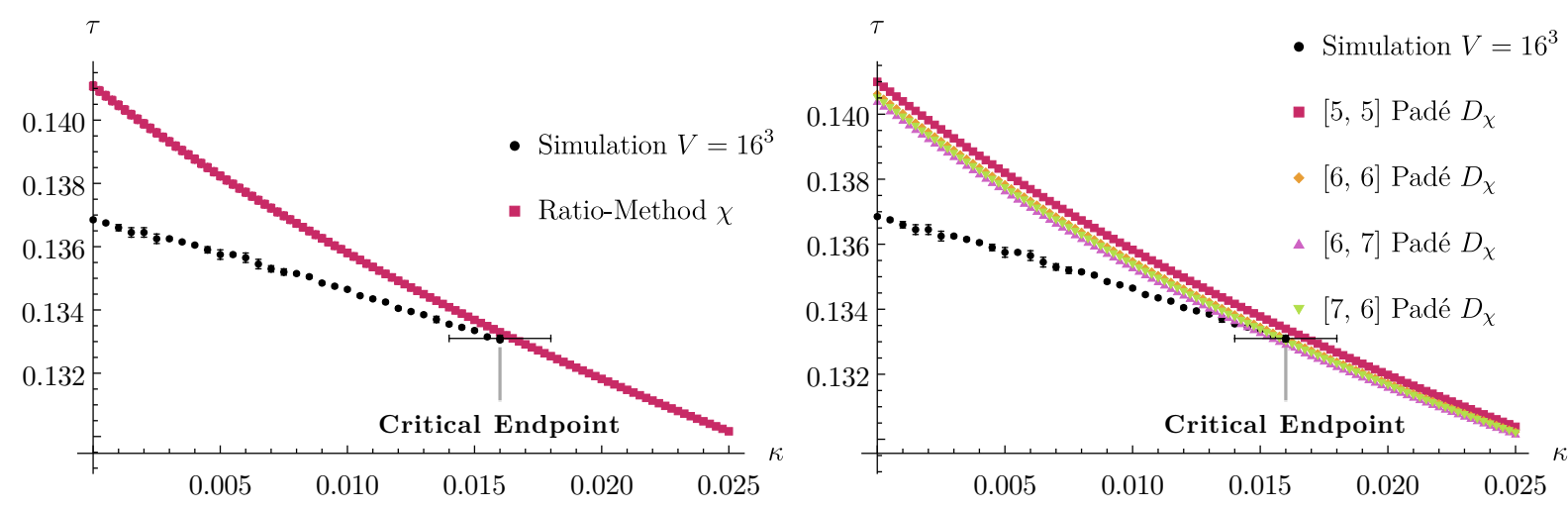

Figure 3.3: Taken from [134]. Radius of convergence at $\mu=0$ from the ratio test (left) and Padé approximants (right). Both methods are compared to the numerically determined phase diagram, with the critical endpoint taken from [68].

critical exponent. Both the ratio test and Padé approximants can only be applied to single variable functions $^{3}$, so we fix both $\kappa$ and $\mu$ before applying them, and then repeat this procedure for different values of $\kappa$ and $\mu$ to obtain estimates for the radius of convergence over a range of parameters.

First, the two methods are applied to $\chi$ for $\mu=0$. From simulations, we know what to expect: a first order transition at $\kappa=0$, that continues to some critical $\kappa_{c}$, forming a first order line ending at a second-order endpoint, after which the transitions are crossovers. The results of the series analysis is shown in figure 3.3. One can observe two problems: First, the critical coupling is systematically over-estimated in the first order region. This can be explained from the fact that the series expansion has information from one phase only and actually determines the end of the metastability region of that phase. In this way, one fails to determine the true critical coupling which needs information from both phases. This phenomenon can be seen in other series expansions too, for example in Potts models [143] with first order transitions and in a Polyakov quark-meson model used for the study of Taylor expansions in $\mu$ for finite density QCD [144]. The

\footnotetext{
${ }^{3}$ We also tested a method which has been developed for multiple variables, namely partial differential approximants in the form used in [142], but did not obtain better results than in the simple approach outlined above.
} 

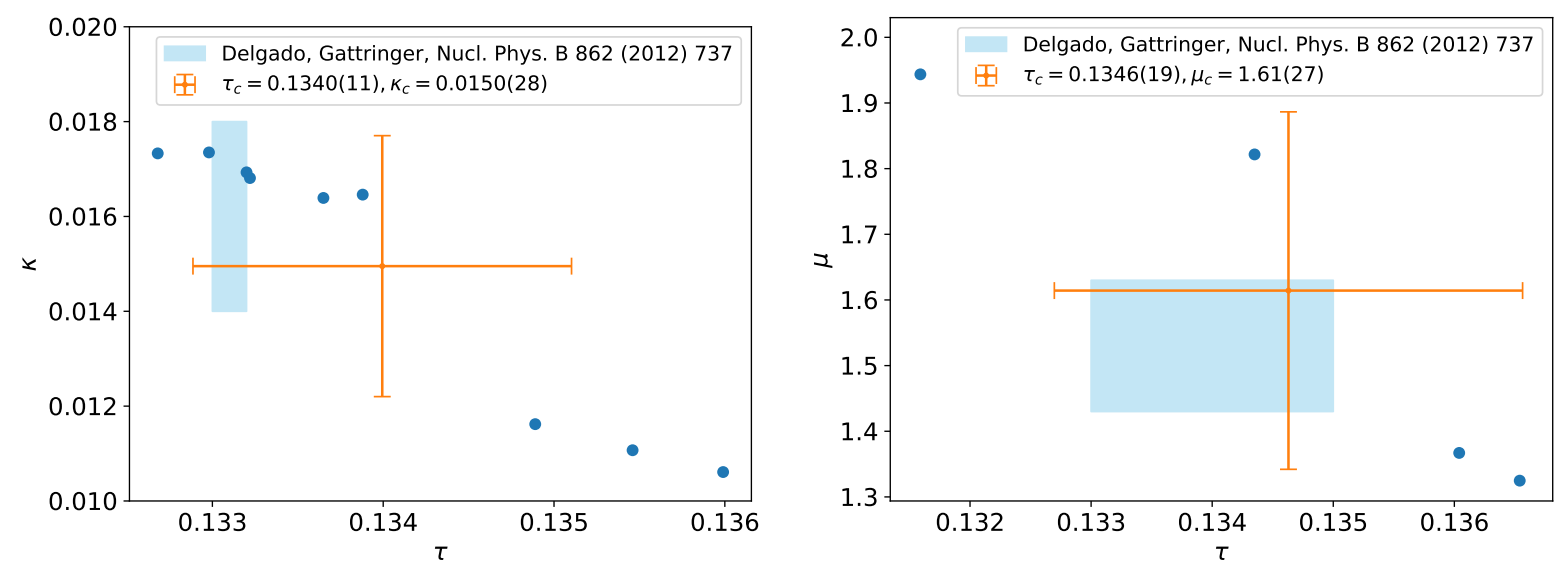

Figure 3.4: Taken from [134]. Estimate for the location of the critical endpoint $\left(\tau_{c}, \kappa_{c}\right)$ at $\mu=0$ (left) and $\left(\tau_{c}, \mu_{c}\right)$ at $\kappa=0.005$ (right). The results are compared to the results from the numerical simulations in [68].

second problem is that while the critical $\tau$ is correctly reproduced at the endpoint, the methods also indicate a finite radius of convergence in the crossover region, where $\chi$ has no singular behavior for real $\tau$. A finite radius of convergence in the crossover region is due to singularities in the complex plane. In principle, real singularities can be detected from the fact that all coefficients of the expansion are positive starting from some order, however, we do not have enough coefficients to estimate the endpoint from this fact. Alternatively it could happen that we do not have enough orders to detect that the radius of convergence actually is infinite. In any case, we cannot directly distinguish crossover behavior from singular behavior of a true phase transition.

Nevertheless we cann locate the critical endpoint by including the specific heat in the analysis. At the critical endpoint, both observables should have a true singularity that can be reproduced faithfully by DLog Padé approximants. Therefore, we use those $\tau$ and $\kappa$ (or $\mu$ if $\kappa$ is fixed) where the poles of DLog Padé approximants of the two observables agree as estimates for the critical endpoint. There can be of course several intersections. Including diagonal Padé approximants from several orders the average of all intersections gives one estimate of the critical endpoint with an error estimate obtained from the scatter. As mentioned above, the residue at the singularity can be used to estimate the critical exponent. As the exponent is expected to be the same for both observables, we judge the quality of an estimate obtained from an intersection by demanding that the corresponding exponents agree within $20 \%$. Excluding the "bad" intersections, we determined the two critical endpoints known from [68], and the results are shown in figure 3.4. Within the error bars, the results agree with those from the flux representation. The series expansion results have roughly $10 \%$ relative error in $\tau_{c}$ and a $20 \%$ relative error in $\kappa_{c}$ and $\mu_{c}$. The larger relative error is probably due to the flatness of the phase diagram in the latter variables, which makes it harder to resolve changes in that direction. Furthermore, the method also delivers an estimate for the critical exponent, that agrees with the true value expected from the universality class of the 3-dimensional Ising model within $\approx 15 \%$. Finally, we remark that the method was also applied successfully to imaginary chemical potential and gives results which are consistent with the tricritical scaling of the $Z(3)$ transition at imaginary $\mu_{i}=i \frac{\pi}{3}$ that is also seen in QCD [145].

Having discussed a tool for the evaluation of effective Polyakov loop theories, we will elucidate some aspects for their derivation next. 



\section{The hopping expansion as a polymer expansion, logarithmic resummations}

In this chapter we will discuss some aspects of the hopping expansion of the fermionic part of QCD that is used in the derivation of the effective theory. Some justification may be necessary what we add in contrast to the previously mentioned references [65, 73, 74].

We clarify how to systematically derive the exponentiated version of the effective theory obtained from the hopping expansion, as discussed in section 4.5 of [146], including corrections which are considered subleading in $N_{\tau}$ in that section. The natural framework to do this is that of polymer expansions, which we introduced in section 2.2. Structurally our approach in doing so is similar to that in $[147,148]$. To facilitate the discussion, we set $\beta=0$, i. e. we work in the strong coupling limit, where the link integration factorizes, a fact that was previously exploited for treatment of staggered fermions in the strong coupling limit $[49,51]$. In contrast to previous publications ${ }^{1}$, we find it more convenient to integrate the spatial gauge fields before integrating the Grassmann-valued fields. This enables us to derive a formula for the effective theory that enables its systematic derivation based on graph rules. In this way we also extend the work of $[73,74]$, where, order by order, only those contributions were derived which have couplings with leading $N_{\tau}$-behavior. Additionally, the graph rules we will derive enable a simpler discussion of resummations, a fact which we will use to discuss how to implement a logarithmic resummation for all contributions. This naturally leads us to the finite cluster method known from condensed matter physics. The finite cluster method should prove a useful tool for the derivation of the effective theory, independent of the specific approach we choose here. For example it should be possible to apply it also when the Grassmann-valued fields are integrated first. Similar to [118], we will contrast the direct evaluation of the partition function against the evaluation of the effective theory, stressing the importance of log-resummations and showing potential new problems when evaluating the effective theory.

\subsection{Deriving the general expression}

As it was explained in section 2.1.2, we split equation (2.12) into temporal and spatial parts in order to achieve a resummation in temporal hoppings. Furthermore, we rewrite the spatial part in order to exploit the fact that the link integration factorizes at strong coupling

$$
\begin{aligned}
-S_{\text {eff }}[W]= & \log \left[\int \mathrm{d}[\Psi, \bar{\Psi}] e^{\bar{\Psi} Q_{\text {stat }}[W] \Psi}\right. \\
& \left.\times \prod_{n \in \Lambda} \prod_{i=1}^{3} \int \mathrm{d} U_{i}(n) e^{\operatorname{tr}\left(R_{i}(n) U_{i}(n)+U_{i}^{\dagger}(n) L_{i}(n)\right)}\right] .
\end{aligned}
$$

\footnotetext{
${ }^{1}$ Except of [149], where parts of the following derivation where covered.
} 
Here, $Q_{\text {stat }}=-1+T$, and $T$ is the $N \times N$ temporal hopping matrix, where $N=4 N_{\tau} N_{s} N_{f} N_{c}$, acting on $N$-dimensional Grassmann valued vectors in the following way ${ }^{2}$

$$
\begin{aligned}
\bar{\Psi}(-1+T) \Psi:= & \sum_{n \in \Lambda}\{-\bar{\Psi}(n) \Psi(n) \\
& +\left[\bar{\Psi}(n) K e^{a \underline{\mu}}\left(1-\gamma_{0}\right) U_{0}(n) \Psi\left(n+e_{0}\right)\right. \\
& \left.\left.+\bar{\Psi}\left(n+e_{0}\right) K e^{-a \underline{\mu}}\left(1+\gamma_{0}\right) U_{0}(n)^{\dagger} \Psi(n)\right]\right\}
\end{aligned}
$$

implying $^{3}$

$$
\begin{aligned}
T(n, m)= & K e^{a \underline{\mu}}\left(1-\gamma_{0}\right) U_{0}(n) \delta_{n_{0}+1, m_{0}}^{-, N_{\tau}} \delta_{\mathbf{n}, \mathbf{m}} \\
& +K e^{-a \mu} \underline{-}\left(1+\gamma_{0}\right) U_{0}(m)^{\dagger} \delta_{n_{0}, m_{0}+1}^{-, N_{\tau}} \delta_{\mathbf{n}, \mathbf{m}} .
\end{aligned}
$$

Since $Q_{\text {stat }}$ is diagonal with respect to spatial arguments, we also introduce the notation

$$
Q_{\text {stat }}(n, m)=: Q_{\text {stat }}\left(n_{0}, m_{0}, \mathbf{n}\right) \delta_{\mathbf{n}, \mathbf{m}} .
$$

Furthermore $R$ and $L$ encode the spatial hops

$$
\begin{aligned}
\operatorname{tr}\left(R_{i}(n) U_{i}(n)\right) & :=\bar{\Psi}(n) K\left(1-\gamma_{i}\right) U_{i}(n) \Psi\left(n+e_{i}\right), \\
\operatorname{tr}\left(U_{i}(n)^{\dagger} L_{i}(n)\right) & :=\bar{\Psi}\left(n+e_{i}\right) K\left(1+\gamma_{i}\right) U_{i}(n)^{\dagger} \Psi(n),
\end{aligned}
$$

which means

$$
\begin{aligned}
R_{i}(n)_{a b} & =\bar{\Psi}(n)_{b} K\left(1-\gamma_{i}\right) \Psi\left(n+e_{i}\right)_{a} \\
L_{i}(n)_{a b} & =\bar{\Psi}\left(n+e_{i}\right)_{b} K\left(1+\gamma_{i}\right) \Psi(n)_{a} .
\end{aligned}
$$

In the last two equations, we used matrix-vector notation in flavor and Dirac space, while the color and spatio-temporal indices were indicated explicitly.

The way things are set up now, we can readily apply equation (C.14) to equation (4.1). For now, we don't need the explicit result of this formula, just the fact that the result of the $\mathrm{d} U_{i}(n)$ integration is some multinomial in $\Psi(n), \Psi\left(n+e_{i}\right), \bar{\Psi}(n)$ and $\bar{\Psi}\left(n+e_{i}\right)$, which we will write as $1+M_{i}(n)$. Since the $\Psi_{\mathrm{s}}$ are Grassmann-valued, this multinomial is finite. Furthermore, the multinomial has the property, that $\Psi$ and $\bar{\Psi}$ come in combinations (as is obvious from the definition of $J$ and $K)$ and therefore $M_{i}(n)$ commutes with $M_{j}(m)$ for all $\{i, j\} \subset\{1,2,3\}$ and $\{n, m\} \subset \Lambda$.

Consequently, equation (4.1) can be rewritten to

$$
-S_{\text {eff }}[W]=\log \left[\int \mathrm{d}[\Psi, \bar{\Psi}] e^{\bar{\Psi} Q_{\text {stat }} \Psi} \prod_{n \in \Lambda} \prod_{i=1}^{3}\left(1+M_{i}(n)\right)\right] .
$$

After expanding the product, the integrals over the Grassmann numbers can be done using Wick's theorem, which we will use for the $N \times N$-matrix $Q$ in the following form:

$$
\int \prod_{k=1}^{N} \mathrm{~d} \Psi_{k} \mathrm{~d} \bar{\Psi}_{k} \prod_{k=1}^{n} \bar{\Psi}_{j_{k}} \Psi_{i_{k}} \exp (\bar{\Psi} Q \Psi)=\operatorname{det}(Q) \sum_{\sigma \in S_{n}} \operatorname{sign}(\sigma) \prod_{k=1}^{n}\left(Q^{-1}\right)_{i_{k} j_{\sigma(k)}}
$$

\footnotetext{
${ }^{2}$ Due to gauge fixing $U_{0}(n)=U_{0}\left(n_{0}, \mathbf{n}\right)=\delta\left(n_{0}=N_{\tau}-1\right) W(\mathbf{n})+\delta\left(n_{0} \neq N_{\tau}-1\right) \mathbb{1}$.

${ }^{3} \delta_{n, m}^{ \pm, N}=( \pm 1)^{\lfloor n / N\rfloor+\lfloor m / N\rfloor} \delta_{n \bmod N, m \bmod N}$
} 
To apply Wick's theorem to equation (4.9), we need the inverse and determinant of $Q_{\text {stat }}$. They are called called the static propagator and the static determinant, have been derived before, see for example [65], and contain the resummations of the temporal hops. The static determinant reads

$$
\begin{aligned}
\operatorname{det}\left(Q_{\text {stat }}\right) & =\prod_{\mathbf{n} \in \Lambda_{s}} \operatorname{det}\left(q_{\text {stat }}(W(\mathbf{n}))\right) \\
& =\prod_{\mathbf{n} \in \Lambda_{s}} \operatorname{det}\left(1+H_{1} W(\mathbf{n})\right)^{2} \operatorname{det}\left(1+\bar{H}_{1} W(\mathbf{n})^{\dagger}\right)^{2} \\
& =\prod_{\mathbf{n} \in \Lambda_{s}} \prod_{f=1}^{N_{f}} \operatorname{det}\left(1+h_{1}^{(f)} W(\mathbf{n})\right)^{2} \operatorname{det}\left(1+\bar{h}_{1}^{(f)} W(\mathbf{n})^{\dagger}\right)^{2}
\end{aligned}
$$

where

$$
\begin{aligned}
H_{1}=\operatorname{diag}\left(h_{1}^{(1)}, \ldots, h_{1}^{\left(N_{f}\right)}\right) & =2 K e^{a \underline{\mu}}, \\
\bar{H}_{1}=\operatorname{diag}\left(\bar{h}_{1}^{(1)}, \ldots, \bar{h}_{1}^{\left(N_{f}\right)}\right) & =2 K e^{-a \underline{\mu}} .
\end{aligned}
$$

In the gauge we chose the static propagator takes a simpler form and reads

$$
\begin{aligned}
Q_{\text {stat }}^{-1}(n, m) & =\delta_{\mathbf{n}, \mathbf{m}} Q_{\text {stat }}^{-1}\left(n_{0}, m_{0}, \mathbf{n}\right) \\
& =\delta_{\mathbf{n}, \mathbf{m}}\left(-\delta_{n_{0}, m_{0}}+\frac{1-\gamma_{0}}{2} B^{\uparrow}\left(n_{0}, m_{0}, \mathbf{n}\right)+\frac{1+\gamma_{0}}{2} B^{\downarrow}\left(n_{0}, m_{0}, \mathbf{n}\right)\right) \\
& =\delta_{\mathbf{n}, \mathbf{m}}\left(-\delta_{n_{0}, m_{0}}+\frac{1}{2} B^{+}\left(n_{0}, m_{0}, \mathbf{n}\right)+\frac{\gamma_{0}}{2} B^{-}\left(n_{0}, m_{0}, \mathbf{n}\right)\right)
\end{aligned}
$$

with

$$
\begin{aligned}
& B^{\uparrow}\left(\tau_{1}, \tau_{2}, \mathbf{n}\right)=\delta\left(\tau_{1} \geq \tau_{2}\right) \frac{H_{1}^{1-\frac{\tau_{1}-\tau_{2}}{N_{\tau}}} W(\mathbf{n})}{1+H_{1} W(\mathbf{n})}-\delta\left(\tau_{2}>\tau_{1}\right) \frac{H_{1}^{\frac{\tau_{2}-\tau_{1}}{N_{\tau}}}}{1+H_{1} W(\mathbf{n})}, \\
& B^{\downarrow}\left(\tau_{1}, \tau_{2}, \mathbf{n}\right)=\delta\left(\tau_{1} \leq \tau_{2}\right) \frac{\bar{H}_{1}^{1-\frac{\tau_{2}-\tau_{1}}{N_{\tau}}} W(\mathbf{n})^{\dagger}}{1+\bar{H}_{1} W(\mathbf{n})^{\dagger}}-\delta\left(\tau_{2}<\tau_{1}\right) \frac{\bar{H}_{1}^{\frac{\tau_{1}-\tau_{2}}{N_{\tau}}}}{1+\bar{H}_{1} W(\mathbf{n})^{\dagger}}, \\
& B^{+}\left(\tau_{1}, \tau_{2}, \mathbf{n}\right)=B^{\uparrow}\left(\tau_{1}, \tau_{2}, \mathbf{n}\right)+B^{\downarrow}\left(\tau_{1}, \tau_{2}, \mathbf{n}\right), \\
& B^{-}\left(\tau_{1}, \tau_{2}, \mathbf{n}\right)=-B^{\uparrow}\left(\tau_{1}, \tau_{2}, \mathbf{n}\right)+B^{\downarrow}\left(\tau_{1}, \tau_{2}, \mathbf{n}\right) .
\end{aligned}
$$

Note that here the potential ambiguity when writing matrices in fractions is no problem, since the numerator commutes with the denominator in all cases. Furthermore, this formula assumes the existence of the inverse of $1+H_{1} W(\mathbf{n})$. There are cases where this is not the case, for example when $H_{1}=1\left(N_{f}=1\right)$ and $W(\mathbf{n})=\operatorname{diag}(1,-1,-1)\left(N_{c}=3\right)$. One can argue that since the left hand side of equation (4.10) is simply a polynomial in the entries of $Q$, one can always shift $H_{1}$ by $\epsilon$ and the limit $\epsilon \rightarrow 0$ must be well defined. However, to organize the expansion properly, we will have to divide by $\operatorname{det}\left(Q_{\text {stat }}\right)$ in equation (4.34), and aspects of this problem are discussed in section 4.6.4.

Similar to equation (3.17) from the weak graph expansion of the spin model, the expansion of the product in equation (4.9) can be represented in terms of graphs. We again interpret the lattice $\Lambda$ as a undirected graph $G_{\Lambda}$ by taking all elements of $\Lambda$ as vertices and the edges connect the nearest neighbors: The graph $G_{\Lambda}$ has the vertices

$$
V_{G_{\Lambda}}=\Lambda
$$


4 The hopping expansion as a polymer expansion, logarithmic resummations

edges

$$
E_{G_{\Lambda}}=\{(n, \mu) \mid n \in \Lambda, \mu \in\{0,1,2,3\}\},
$$

and the edge-to-endpoint function

$$
\sigma_{G_{\Lambda}}((n, \mu))=\left\{n,\left(n+e_{\mu}\right) \bmod \left(N_{\tau}, \mathbf{L}\right)\right\},
$$

where just like before $\mathbf{L}=\left(\sqrt[3]{N_{s}}, \sqrt[3]{N_{s}}, \sqrt[3]{N_{s}}\right)$. An important difference to the weak graph expansion of the spin model is that the inner product in $\prod_{i=1}^{3}$ equation (4.9) does not go over all nearest neighbors in positive direction, but only those which are connected by an edge of the form $(n, i)$ with $i \in\{1,2,3\}$. Consequently, the expansion of the product reads

$$
\prod_{n \in \Lambda} \prod_{i=1}^{3}\left(1+M_{i}(n)\right)=1+\sum_{G \in \mathcal{G}\left(G_{\Lambda}\right)^{\backslash 0}} \prod_{(n, i) \in E_{G}} M_{i}(n),
$$

where $\mathcal{G}^{\backslash 0}\left(G_{\Lambda}\right)$ is the set of all subgraphs of $G_{\Lambda}$, with those subgraphs excluded which contain an edge of the form $(n, 0)$ or which contain an isolated vertex. We stress again that we do not identify isomorphic graphs and disconnected graphs are not excluded from $\mathcal{G}^{\backslash 0}\left(G_{\Lambda}\right)$.

We now want to define graph weights, such that we can apply the polymer expansion formula in a way where connected graphs are the polymers. A first attempt could be

$$
\varphi(G)=\frac{\int \mathrm{d}[\Psi, \bar{\Psi}] e^{\bar{\Psi} Q_{\text {stat }} \Psi} \prod_{(n, i) \in E(G)} M_{i}(n)}{\operatorname{det}\left(Q_{\text {stat }}\right)} .
$$

Note however, that the static propagator in general does not vanish if its temporal arguments are distinct. Consider the disconnected graph $G_{d} \in \mathcal{G}^{\backslash 0}\left(G_{\Lambda}\right)$ that has connected components $G_{1}$ and $G_{2}$ with the property that there are $v_{1} \in V\left(G_{1}\right)$ and $v_{2} \in V\left(G_{2}\right)$ with $v_{1}=\left(\tau_{1}, \mathbf{n}\right)$ and $v_{2}=\left(\tau_{2}, \mathbf{n}\right)$, i. e. $v_{1}$ and $v_{2}$ can be made equal by translating one of them in temporal direction. Then the weight as defined above does not factorize over the connected components of $G_{d}$ :

$$
\varphi\left(G_{d}\right) \neq \varphi\left(G_{1}\right) \varphi\left(G_{2}\right) .
$$

Physically speaking, the weight does not factorize because static quarks can hop in temporal direction.

Therefore, if we would apply the polymer expansion formula now, the polymers would not be connected graphs and the notion of being linked would not coincide with the graph theoretic notion of connectedness. Furthermore, it is quite inconvenient that we have to deal with $\mathcal{G}^{\backslash 0}\left(G_{\Lambda}\right)$ instead of $\mathcal{G}\left(G_{\Lambda}\right)$.

As a remedy for these problems, we organize the expansion slightly different. We again write $\Lambda$ as a product of time slices of spatial lattices $\Lambda=\Lambda_{\tau} \times \Lambda_{s} . \Lambda_{s}$ can be understood as a graph in a completely analogous way to $\Lambda$. We then rewrite the product in the following way:

$$
\begin{aligned}
\prod_{n \in \Lambda} \prod_{i=1}^{3}\left(1+M_{i}(n)\right) & =\prod_{\mathbf{n} \in \Lambda_{s}} \prod_{i=1}^{3} \prod_{\tau \in \Lambda_{\tau}}\left(1+M_{i}(n)\right) \\
& =\prod_{\mathbf{n} \in \Lambda_{s}} \prod_{i=1}^{3} \prod_{\tau=0}^{N_{\tau}-1}\left(1+M_{i}(n)\right) \\
& =\prod_{\mathbf{n} \in \Lambda_{s}} \prod_{i=1}^{3}\left(1+\sum_{k=1}^{N_{\tau}} \sum_{0 \leq \tau_{1}<\cdots<\tau_{k}<N_{\tau}} \prod_{l=1}^{k} M_{i}\left(\tau_{l}, \mathbf{n}\right)\right)
\end{aligned}
$$




$$
\begin{aligned}
& =1+\sum_{G \in \mathcal{G}_{\Lambda_{s}}} \prod_{(\mathbf{n}, i) \in E(G)}\left(\sum_{k=1}^{N_{\tau}} \sum_{0 \leq \tau_{1}<\cdots<\tau_{k}<N_{\tau}} \prod_{l=1}^{k} M_{i}\left(\tau_{l}, \mathbf{n}\right)\right) \\
& =: 1+\sum_{G \in \mathcal{G}_{\Lambda_{s}}} \prod_{(\mathbf{n}, i) \in E(G)} M_{s, i}(\mathbf{n}),
\end{aligned}
$$

where we remind the reader that in section 3.1 we introduced the notation that $\mathcal{G}(G)$ is the set of all subgraphs of $G$ with no isolated vertices and the last step simply defines $M_{s, i}$. Defining the weight of a graph $G \in \mathcal{G}\left(G_{\Lambda_{s}}\right)$ as

$$
\varphi(G)=\frac{\int \mathrm{d}[\Psi, \bar{\Psi}] e^{\bar{\Psi} Q_{\text {stat }} \Psi} \prod_{(\mathbf{n}, i) \in E(G)} M_{s, i}(\mathbf{n})}{\operatorname{det}\left(Q_{\text {stat }}\right)},
$$

we can rewrite this expression using the fact that $Q_{\text {stat }}$ is diagonal with respect to spatial arguments. To facilitate this, we introduce some notation. First, $\Psi(\mathbf{n})$ is the $4 N_{\tau} N_{f} N_{c}$-component vector with

$$
\Psi(\mathbf{n})(\tau)_{\alpha, c}^{f}=\Psi(\tau, \mathbf{n})_{\alpha, c}^{f},
$$

similarly $Q_{\text {stat }}(\mathbf{n})$ is a matrix acting on these vectors and has entries

$$
Q_{\text {stat }}(\mathbf{n})\left(\tau_{1}, \tau_{2}\right)=Q_{\text {stat }}\left(\tau_{1}, \tau_{2}, \mathbf{n}\right)
$$

and finally we define a path integral measure fixed to one spatial point

$$
\mathrm{d}[\Psi(\mathbf{n}), \bar{\Psi}(\mathbf{n})]=\prod_{\tau \in \Lambda_{s}} \prod_{f, \alpha, c} \mathrm{~d} \Psi(\tau, \mathbf{n})_{\alpha, c}^{f}, \bar{\Psi}(\tau, \mathbf{n})_{\alpha, c}^{f} .
$$

Then

$$
\begin{aligned}
\varphi(G)= & \frac{1}{\operatorname{det}\left(Q_{\text {stat }}\right)} \int \mathrm{d}[\Psi, \bar{\Psi}] \prod_{\mathbf{n} \in \Lambda_{s}} e^{\sum_{\tau_{1}, \tau_{2} \in \Lambda_{\tau}} \bar{\Psi}\left(\tau_{1}, \mathbf{n}\right) Q_{\text {stat }}\left(\tau_{1}, \tau_{2}, \mathbf{n}\right) \Psi\left(\tau_{2}, \mathbf{n}\right)} \prod_{(\mathbf{n}, i) \in E(G)} M_{s, i}(\mathbf{n}) \\
= & \frac{1}{\operatorname{det}\left(Q_{\text {stat }}\right)}\left(\prod_{\mathbf{n} \in \Lambda_{s} \backslash V(G)} \int \mathrm{d}[\Psi(\mathbf{n}), \bar{\Psi}(\mathbf{n})] e^{\bar{\Psi}(\mathbf{n}) Q_{\text {stat }}(n) \Psi(\mathbf{n})}\right) \\
& \times \int \prod_{\mathbf{n} \in V(G)} \mathrm{d}[\Psi(\mathbf{n}), \bar{\Psi}(\mathbf{n})] e^{\Psi(\mathbf{n}) Q_{\text {stat }}(\mathbf{n}) \bar{\Psi}(\mathbf{n})} \prod_{(\mathbf{n}, i) \in E(G)} M_{s, i}(\mathbf{n}) \\
= & \frac{1}{\prod_{\mathbf{n} \in V(G)} \operatorname{det}\left(q_{\text {stat }}(W(\mathbf{n}))\right)} \int \prod_{\mathbf{n} \in V(G)} \mathrm{d}[\Psi(\mathbf{n}), \bar{\Psi}(\mathbf{n})] e^{\Psi(\mathbf{n}) Q_{\text {stat }}(\mathbf{n}) \bar{\Psi}(\mathbf{n})} \prod_{(\mathbf{n}, i) \in E(G)} M_{s, i}(\mathbf{n}) .
\end{aligned}
$$

From this it is clear now that for a disconnected graph $G \in \mathcal{G}\left(G_{\Lambda_{s}}\right)$, its weight factorizes over the connected components, i. e. if $G_{1}, \ldots, G_{n}$ are the connected components of $G$, then:

$$
\varphi(G)=\varphi\left(\bigcup_{i=1}^{n} G_{i}\right)=\prod_{i=1}^{n} \varphi\left(G_{i}\right)
$$

The rest now works in analogy to section 3.1. $\mathcal{G}_{c}(G)$ denotes the subset of $\mathcal{G}(G)$ with connected graphs only. We consider the elements of $\mathcal{G}_{c}(G)$ to be polymers and two polymers to be linked if 
4 The hopping expansion as a polymer expansion, logarithmic resummations

their graph union is a connected graph. Starting from equation (4.9) we get

$$
\begin{aligned}
-S_{\text {eff }}\left[U_{0}\right] & \left.=\int \mathrm{d}[\Psi, \bar{\Psi}] e^{\bar{\Psi} \operatorname{det}\left(Q_{\text {stat }}\right) \Psi} \prod_{n \in \Lambda} \prod_{i=1}^{3}\left(1+M_{i}(n)\right)\right) \\
& =\log \left(\int \mathrm{d}[\Psi, \bar{\Psi}] e^{\bar{\Psi} \operatorname{det}\left(Q_{\text {stat }}\right) \Psi}\left[1+\sum_{G \in \mathcal{G}\left(G_{\Lambda_{s}}\right)(\mathbf{n}, i) \in E(G)} M_{s, i}(\mathbf{n})\right]\right) \\
& =\log \left(\operatorname{det}\left(Q_{\text {stat }}\right)\left[1+\sum_{G \in \mathcal{G}\left(G_{\Lambda_{s}}\right)} \varphi(G)\right]\right) \\
& =\log \left(\operatorname{det}\left(Q_{\text {stat }}\right)\right)+\log \left(1+\sum_{n=1}^{\left|\mathcal{G}_{c}\left(G_{\Lambda_{s}}\right)\right|}\left\{\sum_{\left\{G_{1}, \ldots, G_{n}\right\} \in \mathcal{D}_{n}\left(\mathcal{G}_{c}\left(G_{\Lambda_{s}}\right)\right)} \varphi\left(G_{1}\right) \cdots \varphi\left(G_{n}\right)\right)\right.
\end{aligned}
$$

The cluster expansion formula then gives

$$
\begin{aligned}
-S_{\text {eff }}[W]= & \log \left(\operatorname{det}\left(Q_{\text {stat }}\right)\right) \\
& +\sum_{n=1}^{\left|\mathcal{G}_{c}\left(G_{\Lambda_{s}}\right)\right|} \sum_{\left\{G_{1}, \ldots, G_{n}\right\} \in \mathcal{C}_{n}\left(\mathcal{G}_{c}\left(G_{\Lambda_{s}}\right)\right)} \sum_{\left(k_{1}, \ldots, k_{n}\right) \in\left(\mathbb{N}^{*}\right)^{n}} C_{\left(G_{1}, k_{1}\right), \ldots,\left(G_{n}, k_{n}\right)} \varphi\left(G_{1}\right)^{k_{1}} \cdots \varphi\left(G_{n}\right)^{k_{n}}
\end{aligned}
$$

with the expansion coefficients

$$
C_{\left(G_{1}, k_{1}\right), \ldots,\left(G_{n}, k_{n}\right)}=\left.\frac{1}{k_{1} ! \cdots k_{n} !} \frac{\partial^{k_{1}+\cdots+k_{n}} \log \left(P_{\mathrm{eff}}\left(\mathcal{G}_{c}\left(G_{\Lambda_{s}}\right)\right)\right)}{\partial \varphi\left(G_{1}\right)^{k_{1}} \cdots \partial \varphi\left(G_{n}\right)^{k_{n}}}\right|_{\varphi\left(G_{i}\right)=0}
$$

where

$$
P_{\text {eff }}\left(\mathcal{G}_{c}\left(G_{\Lambda_{s}}\right)\right):=1+\sum_{n=1}^{\left|\mathcal{G}_{c}\left(G_{\Lambda_{s}}\right)\right|} \sum_{\left\{G_{1}, \ldots, G_{n}\right\} \in \mathcal{D}_{n}\left(\mathcal{G}_{c}\left(G_{\Lambda_{s}}\right)\right)} \varphi\left(G_{1}\right) \cdots \varphi\left(G_{n}\right) .
$$

Although equation (4.46) is exact in the strong coupling limit, in practice one introduces truncations to the infinite sum by including only contributions up to a certain power in

$$
\kappa=\max _{f \in\left\{1, \ldots, N_{f}\right\}}\left(\kappa^{(f)}\right) .
$$

For practical reasons, it is also important to understand in which cases the application of $\varphi$ to two different graph yields the same result, or at least when the contribution of one graph can be easily obtained from the other. This will be discussed in the next section.

\subsection{Equivalent graph weights}

In many expansion schemes, isomorphic graphs usually have the same weight. However, in our case the graph weights depend on the temporal gauge transporters $W$. Specifically, the weight $\varphi(G)$ of the graph $G$ has a dependence on the $W$ at the vertices $V_{G}$ of $G$. When it is useful, we will highlight this fact in our notation by writing $\varphi(G)\left[V_{G}\right]$. Due to this dependence, isomorphic graphs in general do not have the same weight. However, one might still hope that given an isomorphism 
$(\lambda, \tau)$ between the two graphs $G$ and $G^{\prime}$ one can obtain the weight of $G^{\prime}$ from that of $G$ via

$$
\varphi\left(G^{\prime}\right)=\varphi(G)\left[\lambda\left(V_{G}\right)\right],
$$

i. e. the weight of $G^{\prime}$ is just the weight of $G$ but with $W(\mathbf{n})$ replaced by $W(\lambda(\mathbf{n}))$ for all $\mathbf{n} \in V_{G}$. If the weight of a graph can be obtained from another graph by simply changing the arguments of the occurring $W$ we say that the graphs have equivalent graph weights. In general this is not the case for graph isomorphisms, as we will see explicitly in the next section. The reason is that the calculation of graph weights contains an evaluation of a trace of $\gamma$-matrices. These $\gamma$-matrices lead to a dependence of the graph weights on the relative directions of their edges. These relative directions are in general not respected by graph isomorphisms. They are however respected by translations and symmetry transformations of the graphs which correspond to the isometry group of the cube.

We describe these transformations in more detail. $\Lambda_{s}$ itself can be understood as a group where the group operation is addition modulo the lattice sizes, i. e. this group is isomorphic to $\left(\mathbb{Z} / \sqrt[3]{N_{s}} \mathbb{Z}\right)^{3}$. The natural way to define a group action $\triangleright$ of $\Lambda_{s}$ on itself is that

$$
\mathbf{v}_{1} \triangleright \mathbf{v}_{2}=\mathbf{v}_{1}+\mathbf{v}_{2} \bmod \mathbf{L} .
$$

For the group $O_{h}$ of isometries of a cube, we define a group action on $\Lambda_{s}$ using its usual matrix representation (which we will denote by $\rho$ ) on $\mathbb{R}^{3}$ such that for $T \in O_{h}$

$$
T \triangleright \mathbf{v}=\rho(T)(\mathbf{v}) \bmod \mathbf{L} .
$$

In order not to overburden the notation we use the symbol $\triangleright$ for different group actions.

Every group action on $\Lambda_{s}$ now induces a group action on the graphs $\mathcal{G}_{\Lambda_{s}}$ by defining that for some element $S$ of a group with a group action on $\Lambda_{s}$ the graph $g^{\prime}=S \triangleright g$ has the vertices

$$
V_{g^{\prime}}=S \triangleright V_{g}
$$

and edges

$$
E_{g^{\prime}}=\sigma_{G_{\Lambda}}^{-1}\left(\left\{\left\{S \triangleright \mathbf{v}_{1}, S \triangleright \mathbf{v}_{2}\right\} \mid\left\{\mathbf{v}_{1}, \mathbf{v}_{2}\right\} \in \sigma_{g}\left(E_{g}\right)\right\}\right) .
$$

Just like in the case of the Poincaré group, the two groups $\Lambda_{s}$ and $O_{h}$ can be combined into a group via a semidirect product and the group action of this combined group can simply be defined to be

$$
(T, \mathbf{v}) \triangleright \mathbf{w}=\mathbf{v} \triangleright(T \triangleright \mathbf{w}) .
$$

for $(T, \mathbf{v}) \in O_{h} \rtimes \Lambda_{s}$ and $w \in \Lambda_{s}$. For graph weights one now has that for $g \in \mathcal{G}_{\Lambda_{s}}$ and $g^{\prime}=(T, \mathbf{v}) \triangleright g$ with $(T, \mathbf{v}) \in O_{h} \rtimes \Lambda_{s}$

$$
\varphi\left(g^{\prime}\right)=\varphi(g)\left[V_{g^{\prime}}\right]
$$

When systematically listing graphs up to equivalent weights it is useful to have a method that enables a fast identification of graphs with equivalent weights. To this end one defines a labeling of the vertices that has the property that two graphs with equivalent weights have the same labeling. In order to achieve this, note at first that the vertices of a graph in $\mathcal{G}_{c}$ are a subset of $\Lambda_{s}$, but can also be taken as a subset of $\mathbb{R}^{3}$. The group actions we just discussed can be defined on subsets of $\mathbb{R}^{3}$ completely analogous just without taking modulo. Next one assigns the same mass to every vertex and translate the graph such that its barycenter is at $(0,0,0)$. After that, one applies all symmetry operations of $O_{h}$ and picks the result which minimizes the resulting vertex sets with respect to lexicographical order.

As this discussion was rather abstract, we illustrate it by deriving the effective action to $\mathcal{O}\left(\kappa^{4}\right)$. 


\subsection{Example: Deriving the $\kappa^{4}$-action}

The first step is to determine $M_{i}(n)$ to order $\kappa^{4}$. As every $J$ and $K$ gives one power of $\kappa$, equation (C.14) to $\mathcal{O}\left(\kappa^{4}\right)$ determines $M_{i}(n)$ to be (we will assume that $N_{c} \geq 3$ from now on, for discussions of the effective theory with $N_{c}=2$ see [150])

$$
\begin{aligned}
M_{i}(n)= & \frac{\operatorname{tr}\left(R_{i}(n) L_{i}(n)\right)}{N_{c}}+\frac{\operatorname{tr}\left(R_{i}(n) L_{i}(n)\right)^{2}}{2\left(N_{c}^{2}-1\right)}-\frac{\operatorname{tr}\left(\left[R_{i}(n) L_{i}(n)\right]^{2}\right)}{2 N_{c}\left(N_{c}^{2}-1\right)} \\
& +\delta\left(N_{c} \geq 3\right) \frac{\operatorname{det}\left(R_{i}(n)\right)+\operatorname{det}\left(L_{i}(n)\right)}{N_{c} !}+\mathcal{O}\left(\kappa^{5}\right) .
\end{aligned}
$$

Since every $\Psi\left(\tau_{1}, \mathbf{n}\right)$ has to be paired with a $\bar{\Psi}\left(\tau_{2}, \mathbf{n}\right)$ for the Grassmann integration not to vanish, the term containing the determinants only contributes at $\mathcal{O}\left(\kappa^{6}\right)$ and we will neglect it from now on. From $M_{i}(n)$ one can obtain $M_{s, i}(\mathbf{n})$ :

$$
\begin{aligned}
M_{s, i}(\mathbf{n})= & \sum_{k=1}^{N_{\tau}} \sum_{0 \leq \tau_{1}<\cdots<\tau_{k}<N_{\tau}} \prod_{l=1}^{k} M_{i}\left(\tau_{l}, \mathbf{n}\right) \\
= & \sum_{\tau_{1}=0}^{N_{\tau}-1} M_{i}\left(\tau_{1}, \mathbf{n}\right)+\sum_{0 \leq \tau_{1}<\tau_{2}<N_{t}} M_{i}\left(\tau_{1}, \mathbf{n}\right) M_{i}\left(\tau_{2}, \mathbf{n}\right)+\mathcal{O}\left(\kappa^{6}\right) \\
= & \sum_{\tau_{1}=0}^{N_{\tau}-1}\left(\frac{\operatorname{tr}\left(R_{i}\left(\tau_{1}, \mathbf{n}\right) L_{i}\left(\tau_{1}, \mathbf{n}\right)\right)}{N_{c}}+\frac{\operatorname{tr}\left(R_{i}\left(\tau_{1}, \mathbf{n}\right) L_{i}\left(\tau_{1}, \mathbf{n}\right)\right)^{2}}{2\left(N_{c}^{2}-1\right)}-\frac{\operatorname{tr}\left(\left[R_{i}\left(\tau_{1}, \mathbf{n}\right) L_{i}\left(\tau_{1}, \mathbf{n}\right)\right]^{2}\right)}{2 N_{c}\left(N_{c}^{2}-1\right)}\right) \\
& +\sum_{0 \leq \tau_{1}<\tau_{2}<N_{t}} \frac{\operatorname{tr}\left(R_{i}\left(\tau_{1}, \mathbf{n}\right) L_{i}\left(\tau_{1}, \mathbf{n}\right)\right) \operatorname{tr}\left(R_{i}\left(\tau_{2}, \mathbf{n}\right) L_{i}\left(\tau_{2}, \mathbf{n}\right)\right)}{N_{c}^{2}}+\mathcal{O}\left(\kappa^{5}\right)
\end{aligned}
$$

From this it is clear, that for a graph $G \in \mathcal{G}_{c}$ its contribution is at least of order $\kappa^{2\left|E_{G}\right|}$. Therefore, the relevant subgraphs of $G_{\Lambda_{s}}$ up to $\mathcal{O}\left(\kappa^{4}\right)$ are those with up to two edges. To list them, we at first define:

$$
\begin{aligned}
& \mathbf{n}_{1}=(0,0,0), \\
& \mathbf{n}_{2}=(1,0,0), \\
& \mathbf{n}_{3}=(2,0,0), \\
& \mathbf{n}_{4}=(1,1,0) .
\end{aligned}
$$

Then, the relevant graphs up to equivalent weights are:

- $G_{1}$ with vertices

$$
V_{G_{1}}=\left\{\mathbf{n}_{1}, \mathbf{n}_{2}\right\}
$$

and edges

$$
E_{G_{1}}=\left\{\left(\mathbf{n}_{1}, 1\right)\right\}
$$

For all graphs we list here, the edge-to-endpoint function is simply the edge-to-endpoint function of $G_{\Lambda_{s}}$, restricted to the edges of the subgraph. $G_{1}$ can be visualized in the following way:

$$
G_{1}=\mathbf{n}_{1} \bullet \bullet \mathbf{n}_{2}
$$

- $G_{2}$ with vertices

$$
V_{G_{2}}=\left\{\mathbf{n}_{1}, \mathbf{n}_{2}, \mathbf{n}_{3}\right\},
$$


edges

$$
E_{G_{2}}=\left\{\left(\mathbf{n}_{1}, 1\right),\left(\mathbf{n}_{2}, 1\right)\right\}
$$

and visual representation

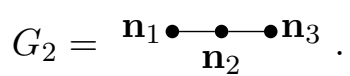

- $G_{3}$ with vertices

$$
V_{G_{3}}=\left\{\mathbf{n}_{1}, \mathbf{n}_{2}, \mathbf{n}_{4}\right\},
$$

edges

$$
E_{G_{3}}=\left\{\left(\mathbf{n}_{1}, 1\right),\left(\mathbf{n}_{2}, 2\right)\right\}
$$

and visual representation

$$
G_{3}=\mathbf{n}_{1} \bullet{\stackrel{\bullet}{\mathbf{n}_{2}}}^{\mathbf{n}_{4}}
$$

Next, we have to compute the weights of these graphs. Applying equation (4.34) to the graphs gives

$$
\begin{aligned}
\varphi\left(G_{1}\right) & =\frac{\int \mathrm{d}[\Psi, \bar{\Psi}] e^{\bar{\Psi} Q_{\text {stat }} \Psi} \prod_{(\mathbf{n}, i) \in E\left(G_{1}\right)} M_{s, i}(\mathbf{n})}{\operatorname{det}\left(Q_{\text {stat }}\right)} \\
& =\frac{\int \mathrm{d}[\Psi, \bar{\Psi}] e^{\bar{\Psi} Q_{\text {stat }} \Psi} M_{s, 1}\left(\mathbf{n}_{1}\right)}{\operatorname{det}\left(Q_{\text {stat }}\right)} \\
& =\frac{1}{\operatorname{det}\left(Q_{\text {stat }}\right)}\left\{\frac{I_{1}}{N_{c}}+\frac{I_{2}}{2\left(N_{c}^{2}-1\right)}-\frac{I_{3}}{2 N_{c}\left(N_{c}^{2}-1\right)}+\frac{I_{4}}{N_{c}^{2}}+\mathcal{O}\left(\kappa^{5}\right)\right\} \\
\varphi\left(G_{2}\right) & =\frac{\int \mathrm{d}[\Psi, \bar{\Psi}] e^{\bar{\Psi} Q_{\text {stat }} \Psi} M_{s, 1}\left(\mathbf{n}_{1}\right) M_{s, 1}\left(\mathbf{n}_{2}\right)}{\operatorname{det}\left(Q_{\text {stat }}\right)} \\
& =\frac{1}{\operatorname{det}\left(Q_{\text {stat }}\right)}\left(\frac{I_{5}}{N_{c}^{2}}+\mathcal{O}\left(\kappa^{5}\right)\right) \\
\varphi\left(G_{3}\right) & =\frac{\int \mathrm{d}[\Psi, \bar{\Psi}] e^{\bar{\Psi} Q_{\text {stat }} \Psi} M_{s, 1}\left(\mathbf{n}_{1}\right) M_{s, 2}\left(\mathbf{n}_{2}\right)}{\operatorname{det}\left(Q_{\text {stat }}\right)} \\
& =\frac{1}{\operatorname{det}\left(Q_{\text {stat }}\right)}\left(\frac{I_{6}}{N_{c}^{2}}+\mathcal{O}\left(\kappa^{5}\right)\right),
\end{aligned}
$$

with the integrals,

$$
\begin{aligned}
I_{1} & =\sum_{\tau_{1}=0}^{N_{\tau}-1} \int \mathrm{d}[\Psi, \bar{\Psi}] e^{\bar{\Psi} Q_{\text {stat }} \Psi} \operatorname{tr}\left(R_{1}\left(\tau_{1}, \mathbf{n}_{1}\right) L_{1}\left(\tau_{1}, \mathbf{n}_{1}\right)\right), \\
I_{2} & =\sum_{\tau_{1}=0}^{N_{\tau}-1} \int \mathrm{d}[\Psi, \bar{\Psi}] e^{\bar{\Psi} Q_{\text {stat }} \Psi} \operatorname{tr}\left(R_{1}\left(\tau_{1}, \mathbf{n}_{1}\right) L_{1}\left(\tau_{1}, \mathbf{n}_{1}\right)\right)^{2}, \\
I_{3} & =\sum_{\tau_{1}=0}^{N_{\tau}-1} \int \mathrm{d}[\Psi, \bar{\Psi}] e^{\bar{\Psi} Q_{\text {stat }} \Psi} \operatorname{tr}\left(\left[R_{1}\left(\tau_{1}, \mathbf{n}_{1}\right) L_{1}\left(\tau_{1}, \mathbf{n}_{1}\right)\right]^{2}\right), \\
I_{4} & =\sum_{0 \leq \tau_{1}<\tau_{2}<N_{\tau}} \int \mathrm{d}[\Psi, \bar{\Psi}] e^{\bar{\Psi} Q_{\text {stat }} \Psi} \operatorname{tr}\left(R_{1}\left(\tau_{1}, \mathbf{n}_{1}\right) L_{1}\left(\tau_{1}, \mathbf{n}_{1}\right)\right) \operatorname{tr}\left(R_{1}\left(\tau_{2}, \mathbf{n}_{1}\right) L_{1}\left(\tau_{2}, \mathbf{n}_{1}\right)\right),
\end{aligned}
$$


4 The hopping expansion as a polymer expansion, logarithmic resummations

$$
\begin{aligned}
& I_{5}=\sum_{\tau_{1}=0}^{N_{\tau}-1} \sum_{\tau_{2}=0}^{N_{\tau}-1} \int \mathrm{d}[\Psi, \bar{\Psi}] e^{\bar{\Psi} Q_{\text {stat }} \Psi} \operatorname{tr}\left(R_{1}\left(\tau_{1}, \mathbf{n}_{1}\right) L_{1}\left(\tau_{1}, \mathbf{n}_{1}\right)\right) \operatorname{tr}\left(R_{1}\left(\tau_{2}, \mathbf{n}_{2}\right) L_{1}\left(\tau_{2}, \mathbf{n}_{2}\right)\right), \\
& I_{6}=\sum_{\tau_{1}=0}^{N_{\tau}-1} \sum_{\tau_{2}=0}^{N_{\tau}-1} \int \mathrm{d}[\Psi, \bar{\Psi}] e^{\bar{\Psi} Q_{\text {stat }} \Psi} \operatorname{tr}\left(R_{1}\left(\tau_{1}, \mathbf{n}_{1}\right) L_{1}\left(\tau_{1}, \mathbf{n}_{1}\right)\right) \operatorname{tr}\left(R_{2}\left(\tau_{2}, \mathbf{n}_{2}\right) L_{2}\left(\tau_{2}, \mathbf{n}_{2}\right)\right) .
\end{aligned}
$$

The integrals are calculated using Wick's theorem. After that one can rewrite the expression in terms of color and Dirac traces, of which the latter can be evaluated to a real number. As the procedure is roughly the same for all integrals and has been implemented on the computer during this thesis, we only show it for the first integral. Note that the fact that $G_{2}$ and $G_{3}$ do not have equivalent graph weights proves the claim made in section 4.2 , that isomorphic graphs in general do not have equivalent graph contributions.

At first, an "unsummed" version of $I_{1}, I_{1}=: \sum_{\tau_{1}=0}^{N_{\tau}-1} I_{1}\left(\tau_{1}\right)$, is computed. We start by inserting the expressions for $R$ and $L$ from equation (4.7) and equation (4.8)

$$
\begin{aligned}
I_{1}\left(\tau_{1}\right)= & \int \mathrm{d}[\Psi, \bar{\Psi}] e^{\bar{\Psi} Q_{\text {stat }} \Psi} \operatorname{tr}\left(R_{1}\left(\tau_{1}, \mathbf{n}_{1}\right) L_{1}\left(\tau_{1}, \mathbf{n}_{1}\right)\right) \\
= & \int \mathrm{d}[\Psi, \bar{\Psi}] e^{\bar{\Psi} Q_{\text {stat }} \Psi} R_{1}\left(\tau_{1}, \mathbf{n}_{1}\right)_{c_{1} c_{2}} L_{1}\left(\tau_{1}, \mathbf{n}_{1}\right)_{c_{2} c_{1}} \\
= & K_{f_{1} f_{2}} K_{f_{3} f_{4}}\left(1-\gamma_{1}\right)_{\alpha_{1} \alpha_{2}}\left(1+\gamma_{1}\right)_{\alpha_{3} \alpha_{4}} \\
& \times \int \mathrm{d}[\Psi, \bar{\Psi}] e^{\bar{\Psi} Q_{\text {stat }} \Psi} \bar{\Psi}\left(\tau_{1}, \mathbf{n}_{1}\right)_{\alpha_{1} c_{2}}^{f_{1}} \Psi\left(\tau_{1}, \mathbf{n}_{2}\right)_{\alpha_{2} c_{1}}^{f_{2}} \bar{\Psi}\left(\tau_{1}, \mathbf{n}_{2}\right)_{\alpha_{3} c_{1}}^{f_{3}} \Psi\left(\tau_{1}, \mathbf{n}_{1}\right)_{\alpha_{4} c_{2}}^{f_{4}} \\
= & -K_{f_{1} f_{2}} K_{f_{3} f_{4}}\left(1-\gamma_{1}\right)_{\alpha_{1} \alpha_{2}}\left(1+\gamma_{1}\right)_{\alpha_{3} \alpha_{4}} \\
& \times \int \mathrm{d}[\Psi, \bar{\Psi}] e^{\bar{\Psi} Q_{\text {stat }} \Psi} \bar{\Psi}\left(\tau_{1}, \mathbf{n}_{1}\right)_{\alpha_{1} c_{2}}^{f_{1}} \Psi\left(\tau_{1}, \mathbf{n}_{1}\right)_{\alpha_{4} c_{2}}^{f_{4}} \bar{\Psi}\left(\tau_{1}, \mathbf{n}_{2}\right)_{\alpha_{3} c_{1}}^{f_{3}} \Psi\left(\tau_{1}, \mathbf{n}_{2}\right)_{\alpha_{2} c_{1}}^{f_{2}}
\end{aligned}
$$

In the next step, Wick's theorem is applied. We remind the reader that only those contractions, where both $\Psi$ and $\bar{\Psi}$ have the same spatial argument, do not vanish.

$$
\begin{aligned}
I_{1}\left(\tau_{1}\right)= & -\operatorname{det}\left(Q_{\text {stat }}\right) K_{f_{1} f_{2}} K_{f_{3} f_{4}}\left(1-\gamma_{1}\right)_{\alpha_{1} \alpha_{2}}\left(1+\gamma_{1}\right)_{\alpha_{3} \alpha_{4}} \\
& \times Q_{\text {stat }}^{-1}\left(\tau_{1}, \tau_{1}, \mathbf{n}_{1}\right)_{\alpha_{4} \alpha_{1}, c_{2} c_{2}}^{f_{f_{1}}} Q_{\text {stat }}^{-1}\left(\tau_{1}, \tau_{1}, \mathbf{n}_{2}\right)_{\alpha_{2} \alpha_{3}, c_{1} c_{1}}^{f_{2} f_{3}} \\
= & -\operatorname{det}\left(Q_{\text {stat }}\right) K_{f_{1} f_{2}} K_{f_{3} f_{4}}\left(1-\gamma_{1}\right)_{\alpha_{1} \alpha_{2}}\left(1+\gamma_{1}\right)_{\alpha_{3} \alpha_{4}} \\
& \times\left(N_{c} \delta_{f_{4} f_{1}} \delta_{\alpha_{4} \alpha_{1}}+\frac{1}{2} \delta_{\alpha_{4} \alpha_{1}} B^{+}\left(\tau_{1}, \tau_{1}, \mathbf{n}_{1}\right)_{c_{2}, c_{2}}^{f_{4} f_{1}}+\frac{1}{2}\left(\gamma_{0}\right)_{\alpha_{4} \alpha_{1}} B^{-}\left(\tau_{1}, \tau_{1}, \mathbf{n}_{1}\right)_{c_{2}, c_{2}}^{f_{4} f_{1}}\right) \\
& \times\left(N_{c} \delta_{f_{2} f_{3}} \delta_{\alpha_{2} \alpha_{3}}+\frac{1}{2} \delta_{\alpha_{2} \alpha_{3}} B^{+}\left(\tau_{1}, \tau_{1}, \mathbf{n}_{2}\right)_{c_{1}, c_{1}}^{f_{2} f_{3}}+\frac{1}{2}\left(\gamma_{0}\right)_{\alpha_{2} \alpha_{3}} B^{-}\left(\tau_{1}, \tau_{1}, \mathbf{n}_{2}\right)_{c_{1}, c_{1}}^{f_{2} f_{3}}\right) \\
= & -\frac{1}{4} \operatorname{det}\left(Q_{\text {stat }}\right) K_{f_{1} f_{2}} K_{f_{3} f_{4}} \operatorname{tr}\left(\left(1-\gamma_{1}\right) \gamma_{0}\left(1+\gamma_{1}\right) \gamma_{0}\right) \\
& \times B^{-}\left(\tau_{1}, \tau_{1}, \mathbf{n}_{1}\right)_{c_{2}, c_{2}}^{f_{4} f_{1}} B^{-}\left(\tau_{1}, \tau_{1}, \mathbf{n}_{2}\right)_{c_{1}, c_{1}}^{f_{2} f_{3}} \\
= & -2 \operatorname{det}\left(Q_{\text {stat }}\right) \sum_{f=1}^{N_{f}}\left(\kappa^{(f)}\right)^{2}\left(W_{1100}^{(f)}\left(\mathbf{n}_{1}\right)-W_{0011}^{(f)}\left(\mathbf{n}_{1}\right)\right)\left(W_{1100}^{(f)}\left(\mathbf{n}_{\mathbf{2}}\right)-W_{0011}^{(f)}\left(\mathbf{n}_{\mathbf{2}}\right)\right) .
\end{aligned}
$$

In the last step we introduced the notation

$$
W_{n_{1} m_{1} n_{2} m_{2}}^{(f)}(\mathbf{n}):=\operatorname{tr}\left(\frac{\left(h_{1}^{(f)} W(\mathbf{n})\right)^{m_{1}}}{\left(1+h_{1}^{(f)} W(\mathbf{n})\right)^{n_{1}}} \frac{\left(\bar{h}_{1}^{(f)} W(\mathbf{n})^{\dagger}\right)^{m_{2}}}{\left(1+\bar{h}_{1}^{(f)} W(\mathbf{n})^{\dagger}\right)^{n_{2}}}\right) .
$$


As the temporal dependence has dropped out, performing the temporal sum is trivial and gives

$$
I_{1}=-2 \operatorname{det}\left(Q_{\text {stat }}\right) N_{\tau} \sum_{f=1}^{N_{f}}\left(\kappa^{(f)}\right)^{2}\left(W_{1100}^{(f)}\left(\mathbf{n}_{\mathbf{1}}\right)-W_{0011}^{(f)}\left(\mathbf{n}_{\mathbf{1}}\right)\right)\left(W_{1100}^{(f)}\left(\mathbf{n}_{\mathbf{2}}\right)-W_{0011}^{(f)}\left(\mathbf{n}_{\mathbf{2}}\right)\right) .
$$

After having obtained all necessary graph weights, the final step is to put everything together according to equation (4.46). To respect all graphs that have equivalent weights to $G_{1}, G_{2}$ and $G_{3}$ we take into account the orbits of these graphs under the group action of $O_{h} \rtimes \Lambda_{s}$. Furthermore it is useful to define two more graphs which have equivalent weights to $G_{1}$ :

- $G_{1}^{\prime}$ with vertices

$$
V_{G_{1}^{\prime}}=\left\{\mathbf{n}_{2}, \mathbf{n}_{3}\right\}
$$

and edges

$$
E_{G_{1}^{\prime}}=\left\{\left(\mathbf{n}_{2}, 1\right)\right\}
$$

- $G_{1}^{\prime \prime}$ with vertices

$$
V_{G_{1}^{\prime \prime}}=\left\{\mathbf{n}_{2}, \mathbf{n}_{4}\right\}
$$

and edges

$$
E_{G_{1}^{\prime \prime}}=\left\{\left(\mathbf{n}_{2}, 2\right)\right\}
$$

To $\mathcal{O}\left(\kappa^{4}\right)$ one then obtains

$$
\begin{aligned}
-S_{\text {eff }}[W]= & \log \left(\operatorname{det}\left(Q_{\text {stat }}\right)\right) \\
& +\sum_{\tilde{G}_{1} \in\left(O_{h} \rtimes \Lambda_{s} \triangleright G_{1}\right)}\left(C_{\left(G_{1}, 1\right)} \varphi\left(\tilde{G}_{1}\right)+C_{\left(G_{1}, 2\right)} \varphi\left(\tilde{G}_{1}\right)^{2}\right) \\
& +C_{\left(G_{1}, 1\right),\left(G_{1}^{\prime}, 1\right)} \sum_{\left(\tilde{G}_{1}, \tilde{G}_{1}^{\prime}\right) \in\left(O_{h} \rtimes \Lambda_{s} \triangleright\left(G_{1}, G_{1}^{\prime}\right)\right)} \varphi\left(\tilde{G}_{1}\right) \varphi\left(\tilde{G}_{1}^{\prime}\right) \\
& +C_{\left(G_{1}, 1\right),\left(G_{1}^{\prime \prime}, 1\right)} \sum_{\left(\tilde{G}_{1}, \tilde{G}^{\prime \prime} 1\right) \in\left(O_{h} \rtimes \Lambda_{s} \triangleright\left(G_{1}, G_{1}^{\prime \prime}\right)\right)} \varphi\left(\tilde{G}_{1}\right) \varphi\left(\tilde{G}^{\prime \prime}{ }_{1}\right) \\
& +C_{\left(G_{2}, 1\right)} \sum_{\tilde{G}_{2} \in\left(O_{h} \rtimes \Lambda_{s} \triangleright G_{2}\right)} \varphi\left(\tilde{G}_{2}\right)+C_{\left(G_{3}, 1\right)} \sum_{\tilde{G}_{3} \in\left(O_{h} \rtimes \Lambda_{s} \triangleright G_{3}\right)} \varphi\left(\tilde{G}_{3}\right),
\end{aligned}
$$

where the Taylor expansion coefficients are determined to be

$$
\begin{aligned}
C_{\left(G_{1}, 1\right)} & =C_{\left(G_{2}, 1\right)}=C_{\left(G_{3}, 1\right)} \\
& =\left.\frac{\mathrm{d}}{\mathrm{d} \alpha} \log \left(1+\left\langle G_{1}\right\rangle \alpha\right)\right|_{\alpha=0} \\
& =\left.\frac{\mathrm{d}}{\mathrm{d} \alpha} \log (1+\alpha)\right|_{\alpha=0} \\
& =1 \\
C_{\left(G_{1}, 2\right)} & =\left.\frac{1}{2 !} \frac{\mathrm{d}^{2}}{\mathrm{~d} \alpha^{2}} \log (1+\alpha)\right|_{\alpha=0} \\
& =-\frac{1}{2 !}, \\
C_{\left(G_{1}, 1\right),\left(G_{1}^{\prime}, 1\right)} & =C_{\left(G_{1}, 1\right),\left(G_{1}^{\prime \prime}, 1\right)}
\end{aligned}
$$


4 The hopping expansion as a polymer expansion, logarithmic resummations

$$
\begin{aligned}
& =\left.\frac{\partial^{2}}{\partial \alpha \partial \beta} \log \left(1+\left\langle G_{1}^{\prime}\right\rangle \alpha+\left\langle G_{1}\right\rangle \beta+\left\langle G_{1}, G_{2}\right\rangle \alpha \beta\right)\right|_{\alpha=\beta=0} \\
& =\left.\frac{\partial^{2}}{\partial \alpha \partial \beta} \log (1+\alpha+\beta)\right|_{\alpha=\beta=0} \\
& =-1 .
\end{aligned}
$$

A bit more loosely, the result can be represented in the following way:

$$
\begin{aligned}
& -S_{\text {eff }}[W]=\log \left(\operatorname{det}\left(Q_{\text {stat }}\right)\right) \\
& +\sum_{\mathbf{n}_{1}, \mathbf{n}_{2}}\left(\varphi\left(\mathbf{n}_{1} \bullet \bullet \mathbf{n}_{2}\right)-\frac{1}{2} \varphi\left(\mathbf{n}_{1} \bullet \bullet \mathbf{n}_{2}\right)^{2}\right) \\
& -\sum_{\mathbf{n}_{1}, \mathbf{n}_{2}, \mathbf{n}_{3}} \varphi\left(\mathbf{n}_{1} \bullet \bullet \mathbf{n}_{2}\right) \varphi\left(\mathbf{n}_{2} \bullet \mathbf{n}_{3}\right)-\sum_{\mathbf{n}_{1}, \mathbf{n}_{2}, \mathbf{n}_{4}} \varphi\left(\mathbf{n}_{1} \bullet \bullet \mathbf{n}_{2}\right) \varphi\left(\stackrel{\bullet \mathfrak{n}_{4}}{\mathbf{n}_{2}}\right)
\end{aligned}
$$

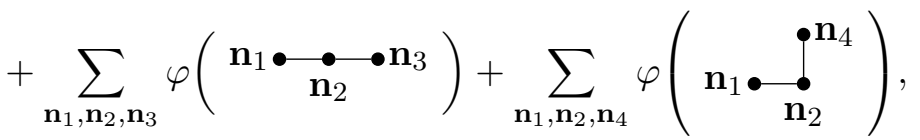

where the summations are understood to respect the relative positions of the $\mathbf{n}_{i}$ and not to cause overcounting of graphs. This finishes the derivation of the effective action to $\mathcal{O}\left(\kappa^{4}\right)$. Note that in contrast to $[65,146]$ we allow for arbitrary number of flavors and the quark masses and chemical potentials can be varied independently. As we will show in the next section, this action can be systematically improved using a logarithmic resummation. We will also argue why it may be preferable to avoid using the action in its current form.

\subsection{Logarithmic resummations}

With the expansion coefficients $C$ coming from a multivariate Taylor expansion of a logarithm, it is not surprising that certain contributions (in fact all) in equation (4.46) can be resummed into a logarithm. One might interject that originally the action was already in a logarithmic form, equation (4.45), but that would miss the point. First of all, in that case we would have to deal with disconnected graphs, and, more importantly, this action is trivial in the sense that when it is used to compute some observable, no higher order terms than the ones obtained inside the logarithm are obtained for this observable. Notably this action is not able to resolve long range interactions necessary for a phase transition and the Polyakov loop correlator is zero for distances that are longer than the truncation. The point here is to obtain logarithms inside the sums over the graphs.

To get started we discuss how to resum the repetition of a single graph weight.

\subsubsection{Resummation of a single graph weight}

The case $n=1$ in the sum on the right side of equation (4.46) reads

$$
\begin{aligned}
\sum_{G \in \mathcal{G}_{c}\left(G_{\Lambda_{s}}\right)} \sum_{k=1}^{\infty} C_{(G, k)} \varphi(G)^{k} & =\left.\sum_{G \in \mathcal{G}_{c}\left(G_{\Lambda_{s}}\right)} \sum_{k=1}^{\infty} \frac{1}{k !} \frac{\mathrm{d}^{k}}{\mathrm{~d} \alpha^{k}} \log (1+\alpha)\right|_{\alpha=0} \varphi(G)^{k} \\
& =\sum_{G \in \mathcal{G}_{c}\left(G_{\Lambda_{s}}\right)} \log (1+\varphi(G)) .
\end{aligned}
$$


The last step was simply an application of Taylor's formula. While it is possible to identify logarithms for $n>2$ in this way, it becomes cumbersome and error-prone relatively quickly. We will discuss a better method in section 4.4.3.

\subsubsection{On the importance of the logarithmic resummation when evaluating the effective theory}

The importance of the logarithmic resummations for the effective theory of Yang-Mills theory was already stressed in [118]. Similar observations can be made in our case.

First, we want to make some general remarks. There are both numerical as well as analytic ways to evaluate the effective theory. Independently of how it is evaluated, the point of the effective theory is that, when it is evaluated, it generates higher order terms in the expansion it is based on, and in that sense it corresponds to a partial resummation of certain terms. Naturally, the effective theory cannot generate all higher order terms but of the higher order terms it generates, they should occur in a direct evaluation of the original theory. This however might not be the case, if the higher order terms would be canceled if one would have included higher order terms in the effective theory before evaluating it. This is exactly what happens if the logarithmic resummation is not included as we will illustrate for a simple example.

Specifically, we consider the $\mathcal{O}\left(\kappa^{2}\right)$ effective action for $N_{f}=1$ with partition function (we assume that $\varphi(G)$ is truncated to $\mathcal{O}\left(\kappa^{2}\right)$ for all $\left.G \in \mathcal{G}_{c}\right)$

$$
\begin{aligned}
Z & =\int \mathrm{d}[W] \operatorname{det}\left(Q_{\text {stat }}\right) \exp \left(\sum_{\tilde{G}_{1} \in\left(O_{h} \rtimes \Lambda_{s} \triangleright G_{1}\right)} \varphi\left(\tilde{G}_{1}\right)\right) \\
& =\int \mathrm{d}[W] \operatorname{det}\left(Q_{\text {stat }}\right) \exp \left(2 \frac{\kappa^{2} N_{\tau}}{N_{c}} \sum_{\langle\mathbf{n}, \mathbf{m}\rangle}\left(W_{1100}(\mathbf{n})-W_{0011}(\mathbf{n})\right)\left(W_{1100}(\mathbf{m})-W_{0011}(\mathbf{m})\right)\right)
\end{aligned}
$$

and compare it to its log-resummed version

$$
\begin{aligned}
Z & =\int \mathrm{d}[W] \operatorname{det}\left(Q_{\text {stat }}\right) \exp \left(\sum_{\tilde{G}_{1} \in\left(O_{h} \rtimes \Lambda_{s} \triangleright G_{1}\right)} \log \left(1+\varphi\left(\tilde{G}_{1}\right)\right)\right) \\
& =\int \mathrm{d}[W] \operatorname{det}\left(Q_{\text {stat }}\right) \prod_{\langle\mathbf{n}, \mathbf{m}\rangle}\left(1+2 \frac{\kappa^{2} N_{\tau}}{N_{c}}\left(W_{1100}(\mathbf{n})-W_{0011}(\mathbf{n})\right)\left(W_{1100}(\mathbf{n})-W_{0011}(\mathbf{n})\right)\right) .
\end{aligned}
$$

Now, when evaluating $Z$ perturbatively, it is clear that for the the first version one will have to obtain the integral

$$
\kappa^{2 k} \int_{\mathrm{SU}\left(N_{c}\right)} \mathrm{d} W \operatorname{det}\left(1+h_{1} W\right)^{2} \operatorname{det}\left(1+\bar{h}_{1} W^{\dagger}\right)^{2} \operatorname{tr}\left(\frac{h_{1} W}{1+h_{1} W}\right)^{k}
$$

for ever higher values of $k \in \mathbb{N}$ when increasing the order of $\kappa$ In contrast to this, the highest $k$ that is necessary for all orders in $\kappa$ is $2 d=6$ for the log-resummed version. This means that higher order corrections in the effective theory lead to the cancellation of certain contributions in the evaluation of the effective theory and these cancellations are missed when the log-resummation is not included.

Furthermore, consider full lattice QCD. When evaluating the partition function in a finite volume, one will always obtain only finite orders in $\kappa$, since the fermion determinant is simply a finite polynomial in a finite volume or, from another viewpoint, there are only a finite number Grassmann 
variables and they are nilpotent. This constraint on the powers of $\kappa$ is however not respected by the effective theory when the logarithmic resummation is not employed. Even in the infinite volume limit, infinite orders of $\kappa$ do not result from a single lattice site (as can happen for the effective theory, see equation (4.119)), but from long range interactions. Using an action without the logarithmic resummations will therefore necessarily lead to violations of the constraint that the square of Grassmann-valued fields vanishes.

\subsubsection{Logarithmic resummation of all graph combinations}

Now that the importance of the logarithmic resummation is clear we discuss how to apply this resummation to all contributions of the effective theory. The method we apply here is basically the finite cluster method, which has been known already a long time and has been applied to other series expansions. For the early works, see [151-153], and for more recent applications we refer to $[154,155]$. In our case there is the additional twist that the graph weights depend on field variables (the $W$ ). The mathematical basis of the finite cluster method is that of Moebius inversion [120].

The idea of the finite cluster method can be applied to our case in the following way: In analogy to section 3.1 one can rewrite equation (4.46)

$$
-S_{\text {eff }}\left[U_{0}\right]=\log \left(\operatorname{det}\left(-1+T\left[U_{0}\right]\right)\right)+\sum_{G \in \mathcal{G}_{c}} \xi(G),
$$

where $\xi(G)$ contains the logarithmic resummation of $G$ and of all connected clusters of subgraphs of $G$. But how can we obtain $\xi(G)$ ?

Getting rid of the contribution from the static determinant using equation (4.48) on writes

$$
\log \left(P_{\text {eff }} \circ \mathcal{G}\left(G_{\Lambda_{s}}\right)\right)=\sum_{G \in \mathcal{G}_{c}\left(G_{\Lambda_{s}}\right)} \xi(G) .
$$

The important point now is, that this equation is in no way limited to be used for the whole spatial lattice graph $G_{\Lambda_{s}}$ only, as the whole derivation would have worked the same if one would have chosen another underlying graph than $G_{\Lambda_{s}}$. In particular, $\varphi(G)$, only depends on the graph topology of $G$, but not on that of $G_{\Lambda_{s}}$, as it is clear from equation (4.40). So, to obtain $\xi$ for some graph $G \in \mathcal{G}_{c}\left(G_{\Lambda_{s}}\right)$ we can use

$$
\log \left(P_{\text {eff }} \circ \mathcal{G}(G)\right)=\sum_{g \in \mathcal{G}_{c}(G)} \xi(g)
$$

and rewrite this to

$$
\xi(G)=\log \left(P_{\text {eff }} \circ \mathcal{G}(G)\right)-\sum_{g \in\left(\mathcal{G}_{c}(G) \backslash G\right)} \xi(g) .
$$

This formula can then be used recursively until one reaches the graphs with equivalent weight to $G_{1}$ as defined in section 4.3. For $G_{1}$ one then has $\mathcal{G}_{c}\left(G_{1}\right)=\left\{G_{1}\right\}$, which is the base case and ends the recursion. Of course, the remarks made about equivalency of graph weights $\varphi$ applies to $\xi$.

The procedure necessitates the computation of $P_{\text {eff }} \circ \mathcal{G}(G)$ for the involved graphs (which are the finite clusters that give the method its name) using equation (4.48). This involves dealing with disconnected graphs which is, however, not a real problem when dealing with graphs that are not as big as $G_{\Lambda_{s}}$. We again illustrate the procedure by log-resumming the $\kappa^{4}$-action equation (4.101). 


\subsubsection{Resummation of the $\kappa^{4}$-action}

Including the log-resummations, the $\kappa^{4}$-action reads in terms of the $\xi$ (we use the more loose notation for readability and the precise expressions should be clear)

$$
\begin{aligned}
& -S_{\text {eff }}[W]=\log \left(\operatorname{det}\left(Q_{\text {stat }}\right)\right) \\
& +\sum_{\mathbf{n}_{1}, \mathbf{n}_{2}} \xi\left(\mathbf{n}_{1} \bullet \bullet \mathbf{n}_{2}\right)
\end{aligned}
$$

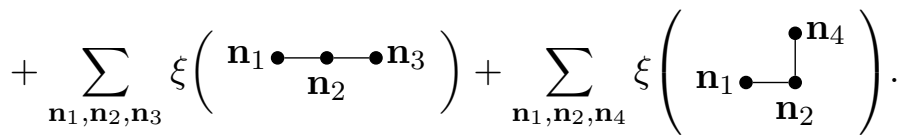

Next we compute the necessary $\xi$ (in the more loose notation, the $\mathcal{G}$ inside the $P_{\text {eff }}$ is omitted in the notation but implied):

$$
\begin{aligned}
& \xi\left(\mathbf{n}_{1} \bullet \bullet \mathbf{n}_{2}\right)=\log \left(P_{\text {eff }}\left(\mathbf{n}_{1} \bullet \bullet \mathbf{n}_{2}\right)\right)
\end{aligned}
$$

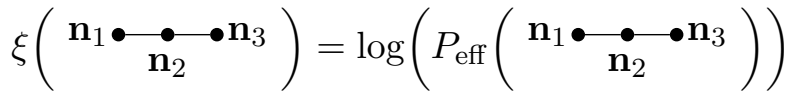

$$
\begin{aligned}
& -\xi\left(\mathbf{n}_{1} \bullet \bullet \mathbf{n}_{2}\right)-\xi\left(\mathbf{n}_{2} \bullet \bullet \mathbf{n}_{3}\right) \\
& =\log \left(P_{\text {eff }}\left(\mathbf{n}_{1} \bullet \mathbf{n}_{2} \bullet \mathbf{n}_{3}\right)\right) \\
& -\log \left(P_{\text {eff }}\left(\mathbf{n}_{1} \bullet \bullet \mathbf{n}_{2}\right)\right)-\log \left(P_{\text {eff }}\left(\mathbf{n}_{2} \bullet \bullet \mathbf{n}_{3}\right)\right) \\
& \xi\left(\mathbf{n}_{1} \bullet \mathfrak{n}^{\mathbf{n}_{4}}\right)=\log \left(P_{\text {eff }}\left(\underset{\mathbf{n}_{1} \bullet \mathfrak{n}^{\mathbf{n}_{4}}}{\mathbf{n}_{2}}\right)\right) \\
& -\xi\left(\mathbf{n}_{1} \bullet \bullet \mathbf{n}_{2}\right)-\xi\left(\begin{array}{l}
\bullet \mathbf{n}_{4} \\
\stackrel{\mathbf{n}_{2}}{ }
\end{array}\right) \\
& =\log \left(P_{\text {eff }}\left(\underset{\mathbf{n}_{2}}{\mathbf{n}_{1} \bullet \mathbf{n}_{4}}\right)\right) \\
& -\log \left(P_{\text {eff }}\left(\mathbf{n}_{1} \bullet \cdot \mathbf{n}_{2}\right)\right)-\log \left(P_{\text {eff }}\left(\begin{array}{l}
\bullet \mathbf{n}_{4} \\
\mathbf{n}_{2}
\end{array}\right)\right)
\end{aligned}
$$

Subsequently, $P_{\text {eff }}$ of the graphs is computed to be

$$
\begin{aligned}
& P_{\text {eff }}\left(\mathbf{n}_{1} \bullet \bullet \mathbf{n}_{2}\right)=1+\varphi\left(\mathbf{n}_{1} \bullet \bullet \mathbf{n}_{2}\right)
\end{aligned}
$$

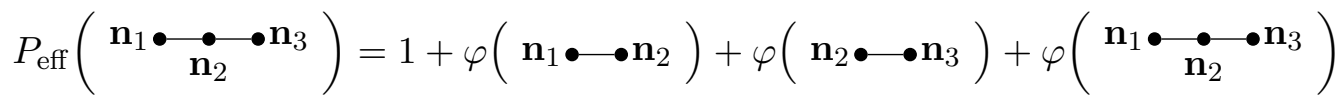

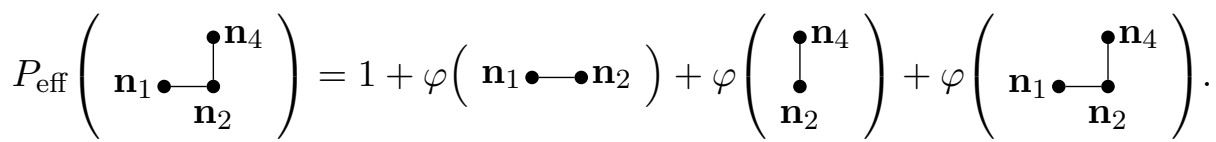

Before we put everything together we remark that although the logarithmic identity

$$
\log (x y)=\log (x)+\log (y)
$$

only holds modulo $2 \pi i$ for complex number we do not have to worry about those extra factors here 
as they will, in the end, always occur inside the exponential function. Keeping this in mind the effective action can be written as

$$
\begin{aligned}
& -S_{\text {eff }}[W]=\log \left(\operatorname{det}\left(Q_{\text {stat }}\right)\right) \\
& +\sum_{\mathbf{n}_{1}, \mathbf{n}_{2}} \log \left(1+\varphi\left(\mathbf{n}_{1} \bullet \bullet \mathbf{n}_{2}\right)\right) \\
& +\sum_{\mathbf{n}_{1}, \mathbf{n}_{2}, \mathbf{n}_{3}} \log \left(\frac{1+\varphi\left(\mathbf{n}_{1} \bullet \bullet \mathbf{n}_{2}\right)+\varphi\left(\mathbf{n}_{2} \bullet \bullet \mathbf{n}_{3}\right)+\varphi\left(\mathbf{n}_{1} \bullet \mathbf{n}_{2} \bullet \mathbf{n}_{3}\right)}{\left(1+\varphi\left(\mathbf{n}_{1} \bullet \bullet \mathbf{n}_{2}\right)\right)\left(1+\varphi\left(\mathbf{n}_{2} \bullet \bullet \mathbf{n}_{3}\right)\right)}\right) \\
& +\sum_{\mathbf{n}_{1}, \mathbf{n}_{2}, \mathbf{n}_{4}} \log \left(\frac{1+\varphi\left(\mathbf{n}_{1} \bullet \bullet \mathbf{n}_{2}\right)+\varphi\left(\stackrel{\bullet \mathbf{n}_{4}}{\dot{\mathbf{n}}_{2}}\right)+\varphi\left(\begin{array}{l}
\mathbf{n}_{1} \bullet \mathbf{n}_{4} \\
\mathbf{n}_{2}
\end{array}\right)}{\left(1+\varphi\left(\mathbf{n}_{1} \bullet \bullet \mathbf{n}_{2}\right)\right)\left(1+\varphi\left(\begin{array}{l}
\bullet \mathbf{n}_{4} \\
\mathbf{n}_{2}
\end{array}\right)\right)}\right) .
\end{aligned}
$$

To avoid violations of the Grassmann constraint it may be necessary to expand the fractions occurring in the logarithms. We note that even if one is not interested in the logarithmic resummed result, the finite cluster method is easier to deal with than with the sums over linked polymers $\mathcal{C}_{n}$ as they occur in equation (4.46). So, instead of using equation (4.46), one should employ the finite cluster method to derive a logarithmic resummed version, and then Taylor expand this result to obtain the answer that would be obtained by using equation (4.46).

According to [156], there are renormalization procedures similar to those of section 3.2.2 for the finite cluster method, but we currently do not understand if they can be applied to our situation. In section 4.4.2 we already saw that it is useful to contrast the evaluation of the effective theory to the direct evaluation of the partition function, and we will explore this in more detail in section 4.6. Before this comparison, we want to discuss another kind of resummation.

\subsection{Ladder resummation}

In this section we investigate how certain contributions to $\varphi\left(G_{1}\right)$ can be resummed. As all graphs are "build up" by $G_{1}$ or those graphs with equivalent weights to it, these resummations concern all other graphs, too. Since all $\Psi$ and $\bar{\Psi}$ that are used for the computation of the weight of $G_{1}$ have either $\mathbf{n}_{1}$ or its nearest neighbor $\mathbf{n}_{2}$ as their spatial arguments, but have the whole range of temporal arguments, these resummations are subsumed under the term ladder resummation, where the metaphorical ladder goes "up" in temporal direction. For simplicity we will assume that $N_{f}=1$ in this section, but the generalization to more flavors should be straightforward.

The main motivation of this section is to make a connection from the logarithmic resummation as explained here to the one given in section 4.7.2 of [74] and to show that the results leading to this connection can be systematically improved.

As the whole ladder resummation itself is too complicated we content ourselves with using the following truncated version of $M_{i}(n)$ (this truncation means that for the integrals over the spatial gauge transporters only the integrals with one $U$ and one $U^{\dagger}$ are considered):

$$
M_{i}(n)=\frac{\operatorname{tr}\left(R_{i}(n) L_{i}(n)\right)}{N_{c}},
$$


leading to a truncated version of $M_{s, i}(\mathbf{n})$ :

$$
M_{s, i}(\mathbf{n})=\sum_{k=1}^{N_{\tau}} \frac{1}{N_{c}^{k}} \sum_{0 \leq \tau_{1}<\cdots<\tau_{k}<N_{\tau}} \prod_{l=1}^{k} \operatorname{tr}\left(R_{i}\left(\tau_{l}, \mathbf{n}\right) L_{i}\left(\tau_{l}, \mathbf{n}\right)\right) .
$$

Consequently, in this truncation scheme the weight for $G_{1}$ reads:

$$
\begin{aligned}
\varphi\left(G_{1}\right) & =\frac{\int \mathrm{d}[\Psi, \bar{\Psi}] e^{\bar{\Psi} Q_{\text {stat }} \Psi} M_{s, 1}\left(\mathbf{n}_{1}\right)}{\operatorname{det}\left(Q_{\text {stat }}\right)} \\
& =\frac{1}{\operatorname{det}\left(Q_{\text {stat }}\right)} \sum_{k=1}^{N_{\tau}} \frac{1}{N_{c}^{k}} \sum_{0 \leq \tau_{1}<\cdots<\tau_{k}<N_{\tau}} \int \mathrm{d}[\Psi, \bar{\Psi}] e^{\bar{\Psi} Q_{\text {stat }} \Psi} \prod_{l=1}^{k} \operatorname{tr}\left(R_{i}\left(\tau_{l}, \mathbf{n}_{1}\right) L_{i}\left(\tau_{l}, \mathbf{n}_{1}\right)\right) .
\end{aligned}
$$

We focus on the integral:

$$
\begin{aligned}
I\left(\tau_{1}, \ldots, \tau_{k}\right):= & \frac{1}{\operatorname{det}\left(Q_{\text {stat }}\right)} \int \mathrm{d}[\Psi, \bar{\Psi}] e^{\bar{\Psi} Q_{\text {stat }} \Psi} \prod_{l=1}^{k} \operatorname{tr}\left(R_{i}\left(\tau_{l}, \mathbf{n}_{1}\right) L_{i}\left(\tau_{l}, \mathbf{n}_{1}\right)\right) \\
= & \frac{\left(-\kappa^{2}\right)^{k}}{\operatorname{det}\left(Q_{\text {stat }}\right)} \prod_{l=1}^{k}\left(1-\gamma_{1}\right)_{\alpha_{l} \beta_{l}}\left(1+\gamma_{1}\right)_{\delta_{l} \epsilon_{l}} \\
& \times \int \mathrm{d}[\Psi, \bar{\Psi}] e^{\bar{\Psi} Q_{\text {stat }} \Psi} \prod_{l=1}^{k} \bar{\Psi}\left(\tau_{l}, \mathbf{n}_{1}\right)_{\alpha_{l} d_{l}} \Psi\left(\tau_{l}, \mathbf{n}_{1}\right)_{\epsilon_{l} d_{l}} \bar{\Psi}\left(\tau_{l}, \mathbf{n}_{2}\right)_{\delta_{l} c_{l}} \Psi\left(\tau_{l}, \mathbf{n}_{2}\right)_{\beta_{l} c_{l}} \\
= & \left(-\kappa^{2}\right)^{k} \prod_{l=1}^{k}\left(1-\gamma_{1}\right)_{\alpha_{l} \beta_{l}}\left(1+\gamma_{1}\right)_{\delta_{l} \epsilon_{l}} \\
& \times \sum_{\sigma, \rho \in S_{k}} \operatorname{sign}(\sigma \circ \rho) \prod_{l=1}^{k} Q_{\text {stat }}^{-1}\left(\tau_{l}, \tau_{\sigma(l)}, \mathbf{n}_{1}\right)_{\epsilon_{l} \alpha_{\sigma(l)}, d_{l} d_{\sigma(l)}} Q_{\text {stat }}^{-1}\left(\tau_{l}, \tau_{\rho(l)}, \mathbf{n}_{2}\right)_{\beta_{l} \delta_{\rho(l)}, c_{l} c_{\rho(l)}} .
\end{aligned}
$$

Since $\left(1-\gamma_{1}\right)\left(1+\gamma_{1}\right)=0$, only the part of $Q_{\text {stat }}^{-1}$ which contains the $\gamma_{0}$ in Dirac space contributes, leading to

$$
\begin{aligned}
I\left(\tau_{1}, \ldots, \tau_{k}\right)= & \left(-\frac{\kappa^{2}}{4}\right)^{k} \sum_{\sigma, \rho \in S_{k}} \operatorname{sign}(\sigma \circ \rho) \prod_{l=1}^{k}\left(1-\gamma_{1}\right)_{\alpha_{l} \beta_{l}}\left(1+\gamma_{1}\right)_{\delta_{l} \epsilon_{l}}\left(\gamma_{0}\right)_{\epsilon_{l} \alpha_{\sigma(l)}}\left(\gamma_{0}\right)_{\beta_{l} \delta_{\rho(l)}} \\
& \times B^{-}\left(\tau_{l}, \tau_{\sigma(l)}, \mathbf{n}_{1}\right)_{d_{l} d_{\sigma(l)}} B^{-}\left(\tau_{l}, \tau_{\rho(l)}, \mathbf{n}_{2}\right)_{c_{l} c_{\rho(l)}} .
\end{aligned}
$$

The $\gamma$-matrices can be rewritten to

$$
\begin{aligned}
\prod_{l=1}^{k}\left(1-\gamma_{1}\right)_{\alpha_{l} \beta_{l}}\left(1+\gamma_{1}\right)_{\delta_{l} \epsilon_{l}}\left(\gamma_{0}\right)_{\epsilon_{l} \alpha_{\sigma(l)}}\left(\gamma_{0}\right)_{\beta_{l} \delta_{\rho(l)}} \\
\quad=\prod_{l=1}^{k}\left(1+\gamma_{1}\right)_{\delta_{l} \epsilon_{l}}\left(\gamma_{0}\right)_{\epsilon_{l} \alpha_{\sigma(l)}}\left(1-\gamma_{1}\right)_{\alpha_{\sigma(l)} \beta_{\sigma(l)}}\left(\gamma_{0}\right)_{\beta_{\sigma(l)} \delta_{\sigma \circ \rho(l)}} \\
=\prod_{l=1}^{k}\left[\left(1+\gamma_{1}\right)\left(\gamma_{0}\right)\left(1-\gamma_{1}\right)\left(\gamma_{0}\right)\right]_{\delta_{l} \delta_{\sigma \circ \rho(l)}} \\
=2^{k} \prod_{l=1}^{k}\left(1+\gamma_{1}\right)_{\delta_{l} \delta_{\sigma \circ \rho(l)}} .
\end{aligned}
$$


Every permutation can be decomposed into disjoint cycles. Therefore, if we define $C_{l}(\sigma)$ to be the number of cycles with length $l$ in the permutation $\sigma$, one obtains:

$$
\begin{aligned}
2^{k} \prod_{l=1}^{k}\left(1+\gamma_{1}\right)_{\delta_{l} \delta_{\sigma \circ \rho(l)}} & =2^{k} \prod_{l=1}^{k} \operatorname{tr}\left(\left(1+\gamma_{1}\right)^{C_{l}(\sigma \circ \rho)}\right) \\
& =2^{k} \prod_{l=1}^{k} 2^{C_{l}(\sigma \circ \rho)+1} \\
& =2^{2 k+C(\sigma \circ \rho)},
\end{aligned}
$$

where $C(\sigma)=\sum_{l=1}^{k} C_{k}(\sigma)$ is simply the total number of disjoint cycles in $\sigma$. Putting this result back into equation (4.142) one obtains

$$
I\left(\tau_{1}, \ldots, \tau_{k}\right)=\left(-\kappa^{2}\right)^{k} \sum_{\sigma, \rho \in S_{k}} \operatorname{sign}(\sigma \circ \rho) 2^{C(\sigma \circ \rho)} \prod_{l=1}^{k} B^{-}\left(\tau_{l}, \tau_{\sigma(l)}, \mathbf{n}_{1}\right)_{d_{l} d_{\sigma(l)}} B^{-}\left(\tau_{l}, \tau_{\rho(l)}, \mathbf{n}_{2}\right)_{c_{l} c_{\rho(l)}} .
$$

We are not aware how the summation over all permutations can be brought to a closed form without further truncations, so we content ourselves with analyzing the contributions of certain permutations only. Specifically, we consider the following cases, which covers all permutations up to $k<3$ :

- $\sigma=\rho=\mathrm{id} \in S_{k}$, then:

$$
\begin{gathered}
\operatorname{sign}(\sigma \circ \rho)=1, \\
2^{C(\sigma \circ \rho)}=2^{C(\mathrm{id})}=2^{k}
\end{gathered}
$$

and

$$
\begin{aligned}
& \prod_{l=1}^{k} B^{-}\left(\tau_{l}, \tau_{\sigma(l)}, \mathbf{n}_{1}\right)_{d_{l} d_{\sigma(l)}} B^{-}\left(\tau_{l}, \tau_{\rho(l)}, \mathbf{n}_{2}\right)_{c_{l} c_{\rho(l)}}= \\
& \quad\left(W_{0011}\left(\mathbf{n}_{1}\right)-W_{1100}\left(\mathbf{n}_{1}\right)\right)^{k}\left(W_{0011}\left(\mathbf{n}_{2}\right)-W_{1100}\left(\mathbf{n}_{2}\right)\right)^{k}
\end{aligned}
$$

- $\sigma=\operatorname{id} \in S_{k}$ and $\rho=(12)(3) \ldots(k)$ in cycle notation, in this case one has:

$$
\begin{gathered}
\operatorname{sign}(\sigma \circ \rho)=-1, \\
2^{C(\sigma \circ \rho)}=2^{C((12)(3) \ldots(k))}=2^{k-1}
\end{gathered}
$$

and, using the fact that $\tau_{1}<\tau_{2}$,

$$
\begin{aligned}
\prod_{l=1}^{k} B^{-} & \left(\tau_{l}, \tau_{\sigma(l)}, \mathbf{n}_{1}\right)_{d_{l} d_{\sigma(l)}} B^{-}\left(\tau_{l}, \tau_{\rho(l)}, \mathbf{n}_{2}\right)_{c_{l} c_{\rho(l)}} \\
= & -\left(W_{0011}\left(\mathbf{n}_{1}\right)-W_{1100}\left(\mathbf{n}_{1}\right)\right)^{k}\left(W_{0011}\left(\mathbf{n}_{2}\right)-W_{1100}\left(\mathbf{n}_{2}\right)\right)^{k-2} \\
& \times\left(W_{2100}\left(\mathbf{n}_{2}\right)+W_{0021}\left(\mathbf{n}_{2}\right)+W_{1010}\left(\mathbf{n}_{2}\right)(2 \kappa)^{2\left(\tau_{2}-\tau_{1}\right)}+W_{1111}\left(\mathbf{n}_{2}\right)(2 \kappa)^{2\left(\tau_{1}-\tau_{2}\right)}\right)
\end{aligned}
$$




$$
\begin{aligned}
= & -\left(W_{0011}\left(\mathbf{n}_{1}\right)-W_{1100}\left(\mathbf{n}_{1}\right)\right)^{k}\left(W_{0011}\left(\mathbf{n}_{2}\right)-W_{1100}\left(\mathbf{n}_{2}\right)\right)^{k-2}\left(W_{2100}\left(\mathbf{n}_{2}\right)+W_{0021}\left(\mathbf{n}_{2}\right)\right) \\
& -(2 \kappa)^{2\left(\tau_{2}-\tau_{1}\right)}\left(W_{0011}\left(\mathbf{n}_{1}\right)-W_{1100}\left(\mathbf{n}_{1}\right)\right)^{k}\left(W_{0011}\left(\mathbf{n}_{2}\right)-W_{1100}\left(\mathbf{n}_{2}\right)\right)^{k-2} W_{1010}\left(\mathbf{n}_{2}\right) \\
& -(2 \kappa)^{2\left(\tau_{1}-\tau_{2}\right)}\left(W_{0011}\left(\mathbf{n}_{1}\right)-W_{1100}\left(\mathbf{n}_{1}\right)\right)^{k}\left(W_{0011}\left(\mathbf{n}_{2}\right)-W_{1100}\left(\mathbf{n}_{2}\right)\right)^{k-2} W_{1111}\left(\mathbf{n}_{2}\right) .
\end{aligned}
$$

- $\rho=\mathrm{id} \in S_{k}$ and $\sigma=(12)(3) \ldots(k)$, this gives the same result as the previous case, but with $\mathbf{n}_{1} \leftrightarrow \mathbf{n}_{2}$

- $\sigma=\rho=(12)(3) \ldots(k)$, now one has $\sigma \circ \rho=$ id so the only difference to the first case is

$$
\begin{aligned}
\prod_{l=1}^{k} B^{-} & \left(\tau_{l}, \tau_{\sigma(l)}, \mathbf{n}_{1}\right)_{d_{l} d_{\sigma(l)}} B^{-}\left(\tau_{l}, \tau_{\rho(l)}, \mathbf{n}_{2}\right)_{c_{l} c_{\rho(l)}} \\
= & \prod_{i=1}^{2}\left(W_{0011}\left(\mathbf{n}_{i}\right)-W_{1100}\left(\mathbf{n}_{i}\right)\right)^{k-2} \\
& \times\left(W_{2100}\left(\mathbf{n}_{i}\right)+W_{0021}\left(\mathbf{n}_{i}\right)+W_{1010}\left(\mathbf{n}_{i}\right)(2 \kappa)^{2\left(\tau_{2}-\tau_{1}\right)}+W_{1111}\left(\mathbf{n}_{i}\right)(2 \kappa)^{2\left(\tau_{1}-\tau_{2}\right)}\right) \\
= & \prod_{i=1}^{2}\left(W_{0011}\left(\mathbf{n}_{i}\right)-W_{1100}\left(\mathbf{n}_{i}\right)\right)^{k-2}\left(W_{2100}\left(\mathbf{n}_{i}\right)+W_{0021}\left(\mathbf{n}_{i}\right)\right) \\
& +W_{1010}\left(\mathbf{n}_{1}\right)\left(\prod_{i=1}^{2}\left(W_{0011}\left(\mathbf{n}_{i}\right)-W_{1100}\left(\mathbf{n}_{i}\right)\right)^{k-2}\right) W_{1111}\left(\mathbf{n}_{2}\right) \\
& +W_{1111}\left(\mathbf{n}_{1}\right)\left(\prod_{i=1}^{2}\left(W_{0011}\left(\mathbf{n}_{i}\right)-W_{1100}\left(\mathbf{n}_{i}\right)\right)^{k-2}\right) W_{1010}\left(\mathbf{n}_{2}\right) \\
& +(2 \kappa)^{2\left(\tau_{2}-\tau_{1}\right)} W_{1010}\left(\mathbf{n}_{1}\right)\left(\prod_{i=1}^{2}\left(W_{0011}\left(\mathbf{n}_{i}\right)-W_{1100}\left(\mathbf{n}_{i}\right)\right)^{k-2}\right)\left(W_{2100}\left(\mathbf{n}_{2}\right)+W_{0021}\left(\mathbf{n}_{2}\right)\right) \\
& +(2 \kappa)^{2\left(\tau_{2}-\tau_{1}\right)}\left(W_{2100}\left(\mathbf{n}_{1}\right)+W_{0021}\left(\mathbf{n}_{1}\right)\right)\left(\prod_{i=1}^{2}\left(W_{0011}\left(\mathbf{n}_{i}\right)-W_{1100}\left(\mathbf{n}_{i}\right)\right)^{k-2}\right) W_{1010}\left(\mathbf{n}_{2}\right) \\
& +(2 \kappa)^{2\left(\tau_{1}-\tau_{2}\right)} W_{1111}\left(\mathbf{n}_{1}\right)\left(\prod_{i=1}^{2}\left(W_{0011}\left(\mathbf{n}_{i}\right)-W_{1100}\left(\mathbf{n}_{i}\right)\right)^{k-2}\right)\left(W_{2100}\left(\mathbf{n}_{2}\right)+W_{0021}\left(\mathbf{n}_{2}\right)\right) \\
& +(2 \kappa)^{2\left(\tau_{1}-\tau_{2}\right)}\left(W_{2100}\left(\mathbf{n}_{1}\right)+W_{0021}\left(\mathbf{n}_{1}\right)\right)\left(\prod_{i=1}^{2}\left(W_{0011}\left(\mathbf{n}_{i}\right)-W_{1100}\left(\mathbf{n}_{i}\right)\right)^{k-2}\right) W_{1111}\left(\mathbf{n}_{2}\right) \\
& +(2 \kappa)^{4\left(\tau_{2}-\tau_{1}\right)} W_{1010}\left(\mathbf{n}_{1}\right)\left(\prod_{i=1}^{2}\left(W_{0011}\left(\mathbf{n}_{i}\right)-W_{1100}\left(\mathbf{n}_{i}\right)\right)^{k-2}\right) W_{1010}\left(\mathbf{n}_{2}\right) \\
& \left.+\prod_{i=1}^{2}\left(W_{0011}\left(\mathbf{n}_{i}\right)-W_{1100}\left(\mathbf{n}_{i}\right)\right)^{k-2}\right) W_{1111}\left(\mathbf{n}_{2}\right) . \\
& W_{1111}\left(\mathbf{n}_{1}\right)\left(\prod_{i}\right)
\end{aligned}
$$

Including the explicit form of the first case ( $\sigma=\rho=\mathrm{id}$ ) in the evaluation of $\varphi\left(G_{1}\right)$, one can easily evaluate the temporal sums for $k=1$, as the temporal dependence drops out for this contribution and a simple combinatorial argument shows

$$
\sum_{0 \leq \tau_{1}<\cdots<\tau_{k}<N_{\tau}}=\left(\begin{array}{c}
N_{\tau} \\
k
\end{array}\right) .
$$


4 The hopping expansion as a polymer expansion, logarithmic resummations

This results in the resummation that establishes the connection to the logarithmic resummation in $[74]$

$$
\begin{aligned}
\varphi\left(G_{1}\right)= & \sum_{k=1}^{N_{\tau}}\left(-\frac{\kappa^{2}}{N_{c}}\right)^{k} \sum_{0 \leq \tau_{1}<\cdots<\tau_{k}<N_{\tau}} \sum_{\sigma, \rho \in S_{k}} \operatorname{sign}(\sigma \circ \rho) 2^{C(\sigma \circ \rho)} \\
& \times \prod_{l=1}^{k} B^{-}\left(\tau_{l}, \tau_{\sigma(l)}, \mathbf{n}_{1}\right)_{d_{l} d_{\sigma(l)}} B^{-}\left(\tau_{l}, \tau_{\rho(l)}, \mathbf{n}_{2}\right)_{c_{l} c_{\rho(l)}} \\
= & \sum_{k=1}^{N_{\tau}}\left(\begin{array}{c}
N_{\tau} \\
k
\end{array}\right)\left(-\frac{2 \kappa^{2}}{N_{c}}\right)^{k}\left(W_{0011}\left(\mathbf{n}_{1}\right)-W_{1100}\left(\mathbf{n}_{1}\right)\right)^{k}\left(W_{0011}\left(\mathbf{n}_{2}\right)-W_{1100}\left(\mathbf{n}_{2}\right)\right)^{k} \\
& +\sum_{k=2}^{N_{\tau}}\left(-\frac{\kappa^{2}}{N_{c}}\right)^{k} \sum_{0 \leq \tau_{1}<\cdots<\tau_{k}<N_{\tau}} \sum_{\sigma, \rho \in S_{k} \backslash\{\operatorname{id}\}} \operatorname{sign}(\sigma \circ \rho) 2^{C(\sigma \circ \rho)} \\
& \times \prod_{l=1}^{k} B^{-}\left(\tau_{l}, \tau_{\sigma(l)}, \mathbf{n}_{1}\right)_{d_{l} d_{\sigma(l)}} B^{-}\left(\tau_{l}, \tau_{\rho(l)}, \mathbf{n}_{2}\right)_{c_{l} c_{\rho(l)}} \\
= & +\left(1-\frac{2 \kappa^{2}}{N_{c}}\left(W_{0011}\left(\mathbf{n}_{1}\right)-W_{1100}\left(\mathbf{n}_{1}\right)\right)\left(W_{0011}\left(\mathbf{n}_{2}\right)-W_{1100}\left(\mathbf{n}_{2}\right)\right)\right) \\
& +\sum_{k=1}^{N_{\tau}}\left(-\frac{\kappa^{2}}{N_{c}}\right)^{k} \sum_{0 \leq \tau_{1}<\cdots<\tau_{k}<N_{\tau} \sigma, \rho \in S_{k} \backslash\{\operatorname{id}\}} \operatorname{sign}(\sigma \circ \rho) 2^{C(\sigma \circ \rho)} \\
& \times \prod_{l=1}^{k} B^{-}\left(\tau_{l}, \tau_{\sigma(l)}, \mathbf{n}_{1}\right)_{d_{l} d_{\sigma(l)}} B^{-}\left(\tau_{l}, \tau_{\rho(l)}, \mathbf{n}_{2}\right)_{c_{l} c_{\rho(l)}} .
\end{aligned}
$$

Including the other cases results in rather lengthy expressions, so we only explain how to obtain them. Generically, the temporal sums and that over $k$ can be written as

$$
S=\sum_{k=2}^{N_{\tau}}\left(\sum_{0 \leq \tau_{1}<\cdots<\tau_{k}<N_{\tau}} \alpha^{\tau_{2}-\tau_{1}}\right) \beta^{k}
$$

for these cases. For the inner sum, the summand does not depend on $\tau_{3}, \ldots, \tau_{k}$. Therefore the summation over these variables simply counts how many values $\tau_{3}, \ldots, \tau_{k}$ can take under the constraint that $\tau_{2}<\tau_{3}<\cdots<\tau_{k}<N_{\tau}$, which is simply $\left(\begin{array}{c}N_{\tau}-\tau_{2}-1 \\ k-2\end{array}\right)$. So, the inner sum can be rewritten to

$$
\begin{aligned}
\left(\sum_{0 \leq \tau_{1}<\cdots<\tau_{k}<N_{\tau}} \alpha^{\tau_{2}-\tau_{1}}\right) & =\sum_{0 \leq<\tau_{1}<\tau_{2}<N_{\tau}-k-2}\left(\begin{array}{c}
N_{\tau}-\tau_{2}-1 \\
k-2
\end{array}\right) \alpha^{\tau_{2}-\tau_{1}} \\
& =\sum_{\tau_{2}=1}^{N_{\tau}-k+1} \sum_{\tau_{1}=0}^{\tau_{2}-1}\left(\begin{array}{c}
N_{\tau}-\tau_{2}-1 \\
k-2
\end{array}\right) \alpha^{\tau_{2}}\left(\frac{1}{\alpha}\right)^{\tau_{1}} \\
& =\frac{\alpha}{\alpha-1} \sum_{\tau_{2}=1}^{N_{\tau}-k+1}\left(\begin{array}{c}
N_{\tau}-\tau_{2}-1 \\
k-2
\end{array}\right)\left(\alpha^{\left.\tau_{2}-1\right)},\right.
\end{aligned}
$$

where we used the well know formula for geometric sums to evaluate the summation over $\tau_{1}$. In principle, this sum can now be expressed in terms of Hypergeometric functions, but it is more 
convenient to notice that

$$
\begin{aligned}
& \sum_{\tau_{2}=1}^{N_{\tau}-k+1}\left(\begin{array}{c}
N_{\tau}-\tau_{2}-1 \\
k-2
\end{array}\right)\left(\alpha^{\tau_{2}}-1\right) \\
& \quad=\left(\begin{array}{c}
N_{\tau}-2 \\
k-2
\end{array}\right)(\alpha-1)+\left(\begin{array}{c}
N_{\tau}-3 \\
k-2
\end{array}\right)\left(\alpha^{2}-1\right)+\cdots+\left(\begin{array}{l}
k-2 \\
k-2
\end{array}\right)\left(\alpha^{N_{\tau}-k+1}-1\right) \\
& \quad=\sum_{\tau_{2}=k-2}^{N_{\tau}-2}\left(\begin{array}{c}
\tau_{2} \\
k-2
\end{array}\right)\left(\alpha^{N_{\tau}-1-\tau_{2}}-1\right) \\
& \quad=\sum_{\tau_{2}=0}^{N_{\tau}-2}\left(\begin{array}{c}
\tau_{2} \\
k-2
\end{array}\right)\left(\alpha^{N_{\tau}-1-\tau_{2}}-1\right)
\end{aligned}
$$

In the last step we used that $\left(\begin{array}{c}N \\ k\end{array}\right)=0$ for $N \geq 0$ and $N<k$. Now the summation over $\tau_{2}$ and $k$ can be interchanged and using the binomial theorem one obtains

$$
\begin{aligned}
S & =\frac{\alpha}{\alpha-1} \sum_{\tau_{2}=0}^{N_{\tau}-2} \sum_{k=2}^{N_{\tau}}\left(\begin{array}{c}
\tau_{2} \\
k-2
\end{array}\right)\left(\alpha^{N_{\tau}-1-\tau_{2}}-1\right) \beta^{k} \\
& =\frac{\alpha \beta^{2}}{\alpha-1} \sum_{\tau_{2}=0}^{N_{\tau}-2}(1+\beta)^{\tau_{2}}\left(\alpha^{N_{\tau}-1-\tau_{2}}-1\right) .
\end{aligned}
$$

The rest is then again just an application of the formula for geometric sums and in total we have

$$
S=\frac{\beta \alpha}{\alpha-1}\left[1-(1+\beta)^{N_{\tau}-1}+\frac{\beta\left(\alpha^{N_{\tau}}-\alpha(1+\beta)^{N_{\tau}-1}\right)}{\alpha-\beta-1}\right],
$$

which completes our discussion of the ladder resummation.

\subsection{Direct evaluation vs evaluation of the effective theory}

The scheme for the derivation of the effective theory we investigated so far can also be used for a perturbative evaluation of $\log (Z)$ around $\kappa_{s}=0$. As we mentioned above, it is useful to compare this direct evaluation to the evaluation of the effective theory. It is clear that when the truncation order for the derivation of the effective theory agrees with the truncation order for the evaluation of the effective theory, both approaches should lead to the same result.

\subsubsection{Direct evaluation of $\log (Z)$}

To obtain $\log (Z)$ instead of $S_{\text {eff }}$, one has to include the integration over the temporal gauge transporters in the right hand side of equation (2.20):

$$
\log (Z)=\log \left(\int \mathrm{d}[W] \mathrm{d}\left[U_{s}\right] \mathrm{d}[\Psi, \bar{\Psi}] e^{-S_{g}[U]-S_{f}[U, \Psi, \bar{\Psi}]}\right) .
$$

Repeating the same steps that lead to equation (4.45) one obtains

$$
\log (Z)=\log \left(\int \mathrm{d}[W] \operatorname{det}\left(Q_{\text {stat }}\right)\left(1+\sum_{n=1}^{\left|\mathcal{G}_{c}\right|} \sum_{\left\{G_{1}, \ldots, G_{n}\right\} \in \mathcal{D}_{n}\left(\mathcal{G}_{c}\left(G_{\Lambda_{s}}\right)\right)} \varphi\left(G_{1}\right) \cdots \varphi\left(G_{n}\right)\right)\right)
$$


4 The hopping expansion as a polymer expansion, logarithmic resummations

$$
=\log \left(\int \mathrm{d}[W] \operatorname{det}\left(Q_{\text {stat }}\right)+\sum_{n=1}^{\left|\mathcal{G}_{c}\right|} \sum_{\left\{G_{1}, \ldots, G_{n}\right\} \in \mathcal{D}_{n}\left(\mathcal{G}_{c}\left(G_{\Lambda_{s}}\right)\right)} \int \mathrm{d}[W] \operatorname{det}\left(Q_{\text {stat }}\right) \varphi\left(G_{1}\right) \cdots \varphi\left(G_{n}\right)\right)
$$

Note that due to the definition of $\mathcal{D}_{n}$, the $\varphi\left(G_{i}\right)$ of one summand do not share any vertices. From equation (4.40) we know that $\varphi\left(G_{i}\right)$ only depends on those $W$ whose argument is a vertex of $G_{i}$ and consequently

$$
\begin{aligned}
\int \mathrm{d}[W] \operatorname{det}\left(Q_{\text {stat }}\right) \varphi\left(G_{1}\right) \cdots \varphi\left(G_{n}\right) & \int \mathrm{d}[W] \prod_{\mathbf{n} \in \Lambda_{s} \backslash V\left(G_{1}\right) \cup \cdots \cup V\left(G_{n}\right)} \operatorname{det}\left(q_{\text {stat }}(W(\mathbf{n}))\right) \\
& \quad \times \prod_{\mathbf{n}_{1} \in V_{G_{1}}} \operatorname{det}\left(q_{\text {stat }}\left(W\left(\mathbf{n}_{1}\right)\right)\right) \varphi\left(G_{1}\right) \cdots \prod_{\mathbf{n}_{n} \in V_{G_{n}}} \operatorname{det}\left(q_{\text {stat }}\left(W\left(\mathbf{n}_{n}\right)\right)\right) \varphi\left(G_{n}\right) \\
= & \left(\int \mathrm{d} W \operatorname{det}\left(q_{\text {stat }}(W)\right)\right)^{N_{s}-\sum_{i=1}^{n}\left|V_{G_{i}}\right|} \\
& \times \prod_{i=1}^{n} \int \prod_{\mathbf{n} \in V_{G_{i}}} \mathrm{~d} W(\mathbf{n}) \operatorname{det}\left(q_{\mathrm{stat}}(W(\mathbf{n}))\right) \varphi\left(G_{i}\right) \\
= & z_{0}^{N_{s}} \prod_{i=1}^{n}\left(\frac{1}{z_{0}}\right)^{\left|V_{G_{i}}\right|} \int \prod_{\mathbf{n} \in V_{G_{i}}} \mathrm{~d} W(\mathbf{n}) \operatorname{det}\left(q_{\mathrm{stat}}(W(\mathbf{n}))\right) \varphi\left(G_{i}\right),
\end{aligned}
$$

where we made the definition

$$
z_{0}:=\int_{\operatorname{SU}\left(N_{c}\right)} \mathrm{d} W \operatorname{det}\left(q_{\mathrm{stat}}(W)\right)
$$

in the last step. Therefore, if we assign to the subgraphs of $\mathcal{G}_{c}$ the weight

$$
\Phi(G)=\left(\frac{1}{z_{0}}\right)^{\left|V_{G}\right|} \int \prod_{\mathbf{n} \in V_{G}} \mathrm{~d} W(\mathbf{n}) \operatorname{det}\left(q_{\mathrm{stat}}(W(\mathbf{n}))\right) \varphi(G),
$$

it again factorizes over the connected components of disconnected graphs and $\log (Z)$ can be written as

$$
\log (Z)=N_{s} \log \left(z_{0}\right)+\log \left(1+\sum_{n=1}^{\left|\mathcal{G}_{c}\right|} \sum_{\left\{G_{1}, \ldots, G_{n}\right\} \in \mathcal{D}_{n}\left(\mathcal{G}_{c}\left(G_{\Lambda_{s}}\right)\right)} \Phi\left(G_{1}\right) \cdots \Phi\left(G_{n}\right)\right) .
$$

The expansion of the logarithm can then be done in the same way as before using the polymer expansion formula, with the additional simplification that graphs, which had equivalent weights in the derivation of the effective theory, now have the same weight. The result of the sum over the graph orbits just gives the number of elements in this orbit and is then similar to the weak embedding number from section 3.1, with the additional constraint that the embeddings are isomorphisms between a graph $g$ and and subgraph of $G_{\Lambda_{s}}$ which has an equivalent weight to $g$. For the graphs of the expansion to $\mathcal{O}\left(\kappa^{4}\right)$ the sums over the orbits give (we assume that $N_{s}$ is large enough so that no graph loops the torus):

$$
\sum_{\tilde{G}_{1} \in\left(O_{h} \rtimes \Lambda_{s} \triangleright G_{1}\right)}=d N_{s}=3 N_{s},
$$




$$
\begin{gathered}
\sum_{\tilde{G}_{2} \in\left(O_{h} \rtimes \Lambda_{s} \triangleright G_{2}\right)}=d N_{s}=3 N_{s}, \\
\sum_{\tilde{G}_{3} \in\left(O_{h} \rtimes \Lambda_{s} \triangleright G_{2}\right)}=2 d(d-1) N_{s}=12 N_{s} .
\end{gathered}
$$

Consequently, the logarithmically resummed partition function to $\mathcal{O}\left(\kappa^{4}\right)$ reads

$$
\begin{aligned}
\log (Z)= & N_{s} \log \left(z_{0}\right) \\
& +3 N_{s} \log \left(1+\Phi\left(\mathbf{n}_{1} \bullet \mathbf{n}_{2}\right)\right) \\
& +3 N_{s} \log \left(\frac{1+2 \Phi\left(\mathbf{n}_{1} \bullet \bullet \mathbf{n}_{2}\right)+\Phi\left(\mathbf{n}_{1} \bullet \mathbf{n}_{2} \bullet \mathbf{n}_{3}\right)}{\left(1+\Phi\left(\mathbf{n}_{1} \bullet \bullet \mathbf{n}_{2}\right)\right)^{2}}\right) \\
& +12 N_{s} \log \left(\frac{1+2 \Phi\left(\mathbf{n}_{1} \bullet \mathbf{n}_{2}\right)+\Phi\left(\mathbf{n}_{1} \bullet \mathbf{n}_{2}^{\mathbf{n}_{4}}\right)}{\left(1+\Phi\left(\mathbf{n}_{1} \bullet \bullet \mathbf{n}_{2}\right)\right)^{2}}\right)
\end{aligned}
$$

Undoing the logarithmic resummation by expanding the partition function to $\mathcal{O}\left(\kappa^{4}\right)$ one obtains, just as expected from equation (4.101):

$$
\begin{aligned}
& \log (Z)=N_{s} \log \left(z_{0}\right) \\
& +3 N_{s}\left(\Phi\left(\mathbf{n}_{1} \bullet \mathbf{n}_{2}\right)-\frac{1}{2} \Phi\left(\mathbf{n}_{1} \bullet \bullet \mathbf{n}_{2}\right)^{2}\right) \\
& -3 N_{s} \Phi\left(\mathbf{n}_{1} \bullet \bullet \mathbf{n}_{2}\right) \Phi\left(\mathbf{n}_{2} \bullet \bullet \mathbf{n}_{3}\right)-12 N_{s} \Phi\left(\mathbf{n}_{1} \bullet \bullet \mathbf{n}_{2}\right) \Phi\left(\begin{array}{l}
\mathfrak{\bullet}_{4} \\
\mathbf{n}_{2}
\end{array}\right) \\
& +3 N_{s} \Phi\left(\underset{\mathbf{n}_{1} \bullet \stackrel{\bullet}{\mathbf{n}_{2}} \bullet \mathbf{n}_{3}}{ }\right)+12 N_{s} \Phi\left(\begin{array}{c}
\mathbf{n}_{1} \bullet \mathbf{n}_{2} \\
\mathbf{n}_{2}
\end{array}\right)
\end{aligned}
$$

To obtain the weights $\Phi$ one has to evaluate $\mathrm{SU}\left(N_{c}\right)$ integrals over the temporal gauge transporters. This will be discussed in section 4.6 .3 and section 6.1 .2 , we at first want to compare the evaluation of the effective theory to the direct evaluation.

\subsubsection{Analytic evaluation of the effective theory}

Since the temporal gauge integrals factorize over the same graphs as the Grassmann integration, it is clear that the effective theory itself can be evaluated using the finite cluster method. For this, one simply ${ }^{4}$ has to enlist for every finite cluster the ways the graphs contained in the effective theory can be embedded into it. As a consistency check, we evaluate the resummed $\kappa^{4}$ action.

\footnotetext{
${ }^{4}$ It is simple in the sense that it is easy to see how this procedure arises from the finite cluster method. However, the practical graph theoretical problem itself is in general an algorithmically hard problem. Unsurprisingly, to $\kappa^{4}$ everything is still rather simple to evaluate.
} 
4 The hopping expansion as a polymer expansion, logarithmic resummations

\section{Evaluation of the resummed $\kappa^{4}$-action}

This should yield the same result as equation (4.186). The logarithm of the normed partition function of the effective theory to $\kappa^{4}$ can be expressed as

$$
\begin{aligned}
\log \left(Z / z_{0}^{N_{s}}\right)= & N_{s} d \Xi_{\kappa^{4}}\left(\mathbf{n}_{1} \bullet \bullet \mathbf{n}_{2}\right)+N_{s} d \Xi_{\kappa^{4}}\left(\mathbf{n}_{1} \bullet \mathbf{n}_{2} \bullet \mathbf{n}_{3}\right) \\
& +N_{s} 2 d(d-1) \Xi_{\kappa^{4}}\left(\underset{\mathbf{n}_{1} \bullet \mathfrak{n}_{2}}{\bullet \mathbf{n}_{4}}\right)
\end{aligned}
$$

where the $\Xi_{\kappa^{4}}$ are calculated following the strategy explained in section 4.4.3. Therefore, we have to evaluate the partition function of the effective theory on the finite clusters defined by the graphs in the previous equation. The partition function on the whole lattice (or any graph that can contain all the graphs of the effective action) reads

$$
\begin{aligned}
& Z=\int \mathrm{d}[W] \prod_{\mathbf{n}} \operatorname{det}\left(q_{\text {stat }}(\mathbf{n})\right) \\
& \times \prod_{\mathbf{n}_{1}, \mathbf{n}_{2}}\left(1+\varphi\left(\mathbf{n}_{1} \bullet \bullet \mathbf{n}_{2}\right)\right)
\end{aligned}
$$

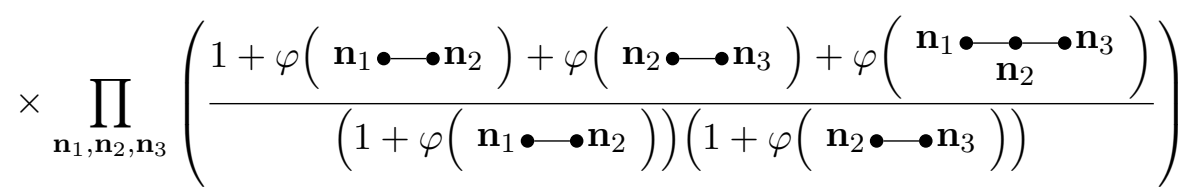

$$
\begin{aligned}
& \times \prod_{\mathbf{n}_{1}, \mathbf{n}_{2}, \mathbf{n}_{4}}\left(\frac{1+\varphi\left(\mathbf{n}_{1} \bullet \mathbf{n}_{2}\right)+\varphi\left(\stackrel{\bullet \mathbf{n}_{4}}{\mathbf{n}_{2}}\right)+\varphi\left(\begin{array}{c}
\bullet \mathbf{n}_{4} \\
\mathbf{n}_{1} \bullet \mathbf{n}_{2}
\end{array}\right)}{\left(1+\varphi\left(\mathbf{n}_{1} \bullet \bullet \mathbf{n}_{2}\right)\right)\left(1+\varphi\left(\stackrel{\bullet \mathbf{n}_{4}}{\mathbf{n}_{2}}\right)\right)}\right) .
\end{aligned}
$$

From this, we can conclude that on the first finite cluster the partition function reads

$$
\begin{aligned}
& \frac{1}{z_{0}^{N_{s}}} Z\left(\mathbf{n}_{1} \bullet \bullet \mathbf{n}_{2}\right) \\
& \quad=\frac{1}{z_{0}^{2}} \int \mathrm{d}[W] \operatorname{det}\left(q_{\text {stat }}\left(W\left(\mathbf{n}_{1}\right)\right)\right) \operatorname{det}\left(q_{\text {stat }}\left(W\left(\mathbf{n}_{2}\right)\right)\right)\left(1+\varphi\left(\mathbf{n}_{1} \bullet \bullet \mathbf{n}_{2}\right)\right) \\
& \quad=1+\frac{1}{z_{0}^{2}} \int \mathrm{d}[W] \operatorname{det}\left(q_{\text {stat }}\left(W\left(\mathbf{n}_{1}\right)\right)\right) \operatorname{det}\left(q_{\text {stat }}\left(W\left(\mathbf{n}_{2}\right)\right)\right) \varphi\left(\mathbf{n}_{1} \bullet \bullet \mathbf{n}_{2}\right) \\
& \quad=1+\Phi\left(\mathbf{n}_{1} \bullet \bullet \mathbf{n}_{2}\right)
\end{aligned}
$$

where we inserted the definition of $\Phi$ according to equation (4.181). The next case is more inter- 
esting

$$
\begin{aligned}
& \frac{1}{z_{0}^{N_{s}}} Z\left(\stackrel{\mathbf{n}_{1} \bullet}{\mathbf{n}_{2}} \bullet \mathbf{n}_{3}\right) \\
& =\frac{1}{z_{0}^{3}} \int \mathrm{d}[W] \operatorname{det}\left(q_{\text {stat }}\left(W\left(\mathbf{n}_{1}\right)\right)\right) \operatorname{det}\left(q_{\text {stat }}\left(W\left(\mathbf{n}_{2}\right)\right)\right) \operatorname{det}\left(q_{\text {stat }}\left(W\left(\mathbf{n}_{3}\right)\right)\right) \\
& \times\left(1+\varphi\left(\mathbf{n}_{1} \bullet \bullet \mathbf{n}_{2}\right)\right)\left(1+\varphi\left(\mathbf{n}_{2} \bullet \bullet \mathbf{n}_{3}\right)\right)
\end{aligned}
$$

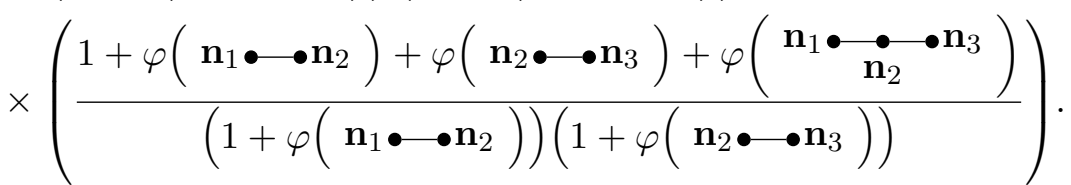

We note now, that the contributions of the two-point coupling cancel against the denominator of the contribution from the three-point coupling and therefore

$$
\begin{aligned}
& \frac{1}{z_{0}^{N_{s}}} Z\left(\underset{\mathbf{n}_{2}}{\bullet} \bullet \mathbf{n}_{3}\right) \\
& =\frac{1}{z_{0}^{3}} \int \mathrm{d}[W] \operatorname{det}\left(q_{\text {stat }}\left(W\left(\mathbf{n}_{1}\right)\right)\right) \operatorname{det}\left(q_{\text {stat }}\left(W\left(\mathbf{n}_{2}\right)\right)\right) \operatorname{det}\left(q_{\text {stat }}\left(W\left(\mathbf{n}_{3}\right)\right)\right)
\end{aligned}
$$

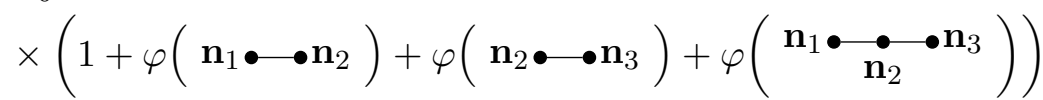

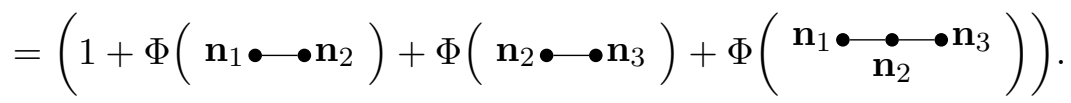

$$
\begin{aligned}
& =\left(1+2 \Phi\left(\mathbf{n}_{1} \bullet \bullet \mathbf{n}_{2}\right)+\Phi\left(\begin{array}{l}
\mathbf{n}_{1} \bullet \stackrel{\bullet}{\mathbf{n}_{2}} \bullet \mathbf{n}_{3} \\
\end{array}\right)\right) .
\end{aligned}
$$

Analogously

$$
\begin{aligned}
& \frac{Z}{z_{0}^{N_{s}}} Z\left(\mathbf{n}_{1} \bullet \stackrel{\bullet}{\mathbf{n}_{2}} \bullet \mathbf{n}_{3}\right) \\
& \quad=\left(1+2 \Phi\left(\mathbf{n}_{1} \bullet \bullet \mathbf{n}_{2}\right)+\Phi\left(\mathbf{n}_{1} \bullet \stackrel{\mathbf{n}_{2}}{\bullet} \cdot \mathbf{n}_{3}\right)\right) .
\end{aligned}
$$

Since $\Xi_{\kappa^{4}}$ and the normed partition function on finite clusters have the same relation as $\xi$ and $P_{\text {eff }}$ in section 4.4 .4 we obtain

$$
\begin{aligned}
& \Xi_{\kappa^{4}}\left(\mathbf{n}_{1} \bullet \bullet \mathbf{n}_{2}\right)=\log \left(1+\Phi\left(\mathbf{n}_{1} \bullet \bullet \mathbf{n}_{2}\right)\right),
\end{aligned}
$$

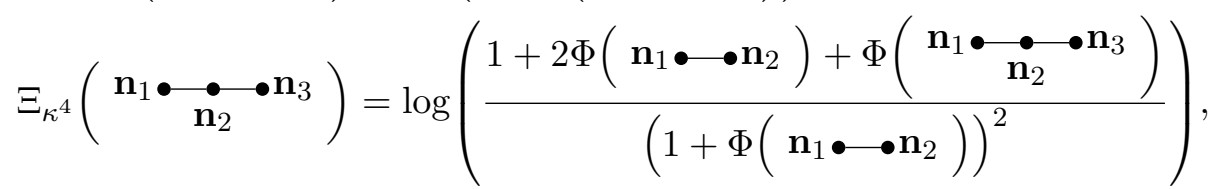

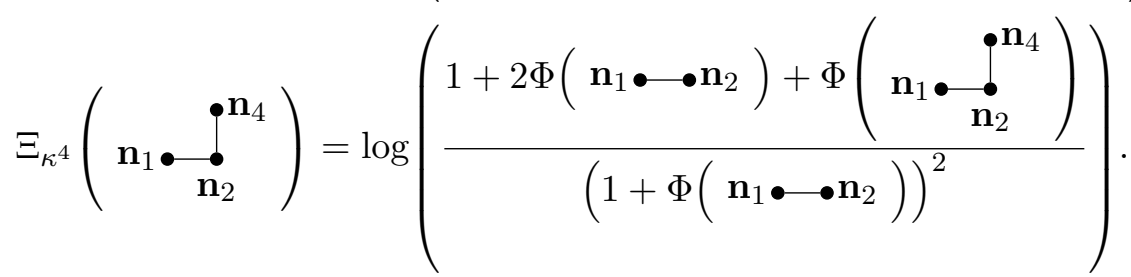

Inserting these equations in equation (4.188) leads to equation (4.186), just like expected.

The cancellations that are necessary to obtain the correct result cannot occur when only the $\kappa^{2}$ 
4 The hopping expansion as a polymer expansion, logarithmic resummations

action is used. Do we run into a similar problem as the one explained in section 4.4.2?

\section{Evaluation of the resummed $\kappa^{2}$-action}

Just like for the $\kappa^{4}$ the logarithm of the normed partition function has the generic form

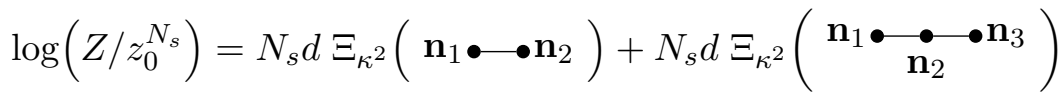

$$
\begin{aligned}
& +N_{s} d \Xi_{\kappa^{2}}\left(\begin{array}{cc} 
& \bullet \mathbf{n}_{4} \\
\mathbf{n}_{1} \bullet & \bullet \\
& \mathbf{n}_{2}
\end{array}\right) .
\end{aligned}
$$

The specific form of the partition function is different however

$$
Z=\int \mathrm{d}[W] \prod_{\mathbf{n}} \operatorname{det}\left(q_{\mathrm{stat}}(\mathbf{n})\right) \prod_{\mathbf{n}_{1}, \mathbf{n}_{2}}\left(1+\varphi\left(\mathbf{n}_{1} \bullet \bullet \mathbf{n}_{2}\right)\right) .
$$

Evaluating the partition function on the finite cluster $\bullet$ - gives the same result as in the $\kappa^{4}$ case. For the next cluster one obtains

$$
\begin{aligned}
\frac{1}{z_{0}^{N_{s}}} Z( & \left.\mathbf{n}_{1} \bullet \mathbf{n}_{2} \bullet \mathbf{n}_{3}\right) \\
= & \frac{1}{z_{0}^{3}} \int \mathrm{d}[W] \operatorname{det}\left(q_{\text {stat }}\left(W\left(\mathbf{n}_{1}\right)\right)\right) \operatorname{det}\left(q_{\text {stat }}\left(W\left(\mathbf{n}_{2}\right)\right)\right) \operatorname{det}\left(q_{\text {stat }}\left(W\left(\mathbf{n}_{3}\right)\right)\right) \\
& \times\left(1+\varphi\left(\mathbf{n}_{1} \bullet \bullet \mathbf{n}_{2}\right)\right)\left(1+\varphi\left(\mathbf{n}_{2} \bullet \bullet \mathbf{n}_{3}\right)\right) . \\
= & 1+2 \Phi\left(\mathbf{n}_{1} \bullet \bullet \mathbf{n}_{2}\right) \\
& +\frac{1}{z_{0}^{3}} \int \mathrm{d}[W]\left(\prod_{i=1}^{3} \operatorname{det}\left(q_{\text {stat }}\left(W\left(\mathbf{n}_{i}\right)\right)\right)\right) \varphi\left(\mathbf{n}_{1} \bullet \bullet \mathbf{n}_{2}\right) \varphi\left(\mathbf{n}_{2} \bullet \bullet \mathbf{n}_{3}\right) .
\end{aligned}
$$

What do we have now? In contrast to the fully consistent calculation to $\kappa^{4}$, we are missing the contribution

$$
\Phi\left(\stackrel{\mathbf{n}_{1} \bullet \mathbf{n}_{2} \bullet \mathbf{n}_{3}}{\bullet}\right),
$$

which is acceptable, however we also generate a contribution which seemingly does not come up in the full calculation, namely

$$
\frac{1}{z_{0}^{3}} \int \mathrm{d}[W]\left(\prod_{i=1}^{3} \operatorname{det}\left(q_{\mathrm{stat}}\left(W\left(\mathbf{n}_{i}\right)\right)\right) \varphi\left(\mathbf{n}_{1} \bullet \bullet \mathbf{n}_{2}\right) \varphi\left(\mathbf{n}_{2} \bullet \bullet \mathbf{n}_{3}\right),\right.
$$

which would be problematic.

Then again, the $\varphi$ and $\Phi$ are quite complicated objects and a closer look reveals the following: To the orders relevant here, using equations (4.76) and (4.78) with $I_{1}$ and $I_{5}$ inserted from equations (4.81) and (4.85) one obtains

$$
\begin{aligned}
\varphi & \left(\mathbf{n}_{1} \bullet \bullet \mathbf{n}_{2}\right) \\
& =\frac{1}{N_{c} \operatorname{det}\left(Q_{\text {stat }}\right)} \sum_{\tau_{1}=0}^{N_{\tau}-1} \int \mathrm{d}[\Psi, \bar{\Psi}] e^{\bar{\Psi} Q_{\text {stat }} \Psi} \operatorname{tr}\left(R_{1}\left(\tau_{1}, \mathbf{n}_{1}\right) L_{1}\left(\tau_{1}, \mathbf{n}_{1}\right)\right),
\end{aligned}
$$




$$
\begin{aligned}
& \varphi\left(\mathbf{n}_{1} \bullet \dot{\mathbf{n}}_{2} \bullet \mathbf{n}_{3}\right) \\
& \quad=\frac{1}{N_{c}^{2} \operatorname{det}\left(Q_{\text {stat }}\right)} \sum_{\tau_{1}=0}^{N_{\tau}-1} \sum_{\tau_{2}=0}^{N_{\tau}-1} \int \mathrm{d}[\Psi, \bar{\Psi}] e^{\bar{\Psi} Q_{\text {stat }} \Psi} \operatorname{tr}\left(R_{1}\left(\tau_{1}, \mathbf{n}_{1}\right) L_{1}\left(\tau_{1}, \mathbf{n}_{1}\right)\right) \operatorname{tr}\left(R_{1}\left(\tau_{2}, \mathbf{n}_{2}\right) L_{1}\left(\tau_{2}, \mathbf{n}_{2}\right)\right) .
\end{aligned}
$$

Applying Wick's theorem to evaluate the second integral, one obtains contractions, which mix the Grassmann numbers of the two traces and contractions, that don't. The latter lead to the first integral and a translated variant of it, or, in other words

$$
\varphi\left(\mathbf{n}_{1} \bullet \mathbf{n}_{2} \bullet \mathbf{n}_{3}\right)=\varphi\left(\mathbf{n}_{1} \bullet \mathbf{n}_{2}\right) \varphi\left(\mathbf{n}_{1} \bullet \bullet \mathbf{n}_{2}\right)+\text { mixing contractions }
$$

Therefore equation (4.206) corresponds to parts of equation (4.205) and the $\kappa^{2}$ action correctly predicts certain $\kappa^{4}$ contributions of the full calculation and produces no $\kappa^{4}$-contributions which do not come up in the full calculation. Similar observation can certainly be made at higher orders, but we mention that there is still one more caveat, which we will discuss at the end of the next section.

\subsubsection{Evaluation of the temporal gauge integrals for the case $N_{c}=3$}

After sorting out the combinatorics of the expansion, the problem of determining the partition function reduces to (both in the direct evaluation and the evaluation of the effective theory) solving single site $\mathrm{SU}\left(N_{c}\right)$ integrals. In the real world, one has $N_{c}=3$, so we will describe how to solve the integrals for this case here, generalizations to arbitrary number of colors will be discussed in section 6.1.2. The integrands are the static determinant at a single site $\operatorname{det}\left(q_{\mathrm{stat}}(W)\right)$ and furthermore, inspecting the static propagator, one also has products of traces of the form

$$
\operatorname{tr}\left(\frac{W^{m}}{\prod_{i=1}^{N_{f}}\left(1+h_{1}^{\left(f_{i}\right)} W\right)^{n_{1, i}}\left(1+\bar{h}_{1}^{\left(f_{i}\right)} W^{\dagger}\right)^{n_{2, i}}}\right) .
$$

One possibility to solve these integrals is to convert all involved quantities to Polyakov loops $L=\operatorname{tr}(W)$ and their complex conjugate and then use the formula provided in [67] for integrals of the type

$$
\int_{\mathrm{SU}(3)} \mathrm{d} W L^{n}\left(L^{*}\right)^{m} .
$$

The formula for the conversion of the static determinant is given, for example, in [65]. For the conversion of the traces, previously generating functionals were used [73], for our more general case these generating functionals do not work. It may be possible to derive more general generating functionals, there is another way however.

The characteristic polynomial $p(\lambda)=\operatorname{det}(\lambda-A)$ of a general $3 \times 3$ matrix $A$ reads (this can be shown by using the formal identity $\operatorname{det}(A)=\exp (\operatorname{tr}(\log (A))))$

$$
p(\lambda)=\lambda^{3}-\lambda^{2} \operatorname{tr}(A)+\lambda \frac{1}{2}\left(\operatorname{tr}(A)^{2}-\operatorname{tr}\left(A^{2}\right)\right)-\operatorname{det}(A) .
$$

According to the Cayley-Hamilton theorem this means that

$$
A^{3}-A^{2} \operatorname{tr}(A)+A \frac{1}{2}\left(\operatorname{tr}(A)^{2}-\operatorname{tr}\left(A^{2}\right)\right)-\operatorname{det}(A)=0,
$$


4 The hopping expansion as a polymer expansion, logarithmic resummations

from which one can conclude that for non-singular $A$

$$
A^{-1}=\frac{1}{\operatorname{det}(A)}\left(A^{2}-A \operatorname{tr}(A)+\frac{1}{2}\left(\operatorname{tr}(A)^{2}-\operatorname{tr}\left(A^{2}\right)\right)\right) .
$$

Furthermore, for $W \in \mathrm{SU}(3)$ specifically one has

$$
\operatorname{tr}\left(W^{-1}\right)=\operatorname{tr}\left(W^{\dagger}\right)=\operatorname{tr}(W)^{*}=\frac{1}{2}\left(\operatorname{tr}(W)^{2}-\operatorname{tr}\left(W^{2}\right)\right)
$$

and

$$
\operatorname{tr}\left(W^{n+1}\right)=\operatorname{tr}\left(W^{n}\right) \operatorname{tr}(W)-\operatorname{tr}\left(W^{n-1}\right) \operatorname{tr}(W)^{*}+\operatorname{tr}\left(W^{n-2}\right),
$$

which gives a recursion relation to convert traces of powers of $W$ into Polyakov loops and their complex conjugate. Solving this recursion relation (which is a standard problem in mathematics) leads to Lucas Polynomials [157]. Putting everything together the inverse matrices which occur in equation (4.210) can be reexpressed as

$$
\begin{aligned}
& \frac{1}{1+h_{1} W}= \frac{1+h_{1}(L-W)+h_{1}^{2}\left(L^{*}-W L+W^{2}\right)}{1+h_{1} L+h_{1}^{2} L^{*}+h_{1}^{3}} \\
& \frac{1}{1+\bar{h}_{1} W^{\dagger}}=\frac{1+\bar{h}_{1}\left(L^{*}-W^{\dagger}\right)+\bar{h}_{1}^{2}\left(L-W^{\dagger} L^{*}+\left(W^{\dagger}\right)^{2}\right)}{1+\bar{h}_{1} L^{*}+\bar{h}_{1}^{2} L+\bar{h}_{1}^{3}} .
\end{aligned}
$$

Therefore the trace corresponds to ratio of multinomials in $L$ and $L^{*}$. For the complete integrand, the denominators partially cancel against the static determinant and the residual expression can be expanded in $h_{1}^{(f)}$ and $\bar{h}_{1}^{(f)}$.

For the direct evaluation, this expansion terminates at finite powers of $h_{1}^{(f)}$ and $\bar{h}_{1}^{(f)}$. To see that this is the case, we insert equation (4.40) into equation (4.181) to obtain

$$
\Phi(G)=\left(\frac{1}{z_{0}}\right)^{\left|V_{G}\right|} \int \prod_{\mathbf{n} \in V_{G}} \mathrm{~d} W(\mathbf{n})\left(\int \prod_{\mathbf{n} \in V(G)} \mathrm{d}[\Psi(\mathbf{n}), \bar{\Psi}(\mathbf{n})] e^{\Psi(\mathbf{n}) Q_{\text {stat }}(\mathbf{n}) \bar{\Psi}(\mathbf{n})} \prod_{(\mathbf{n}, i) \in E(G)} M_{s, i}(\mathbf{n})\right) .
$$

Due to the nilpotency of the Grassmann numbers, the integrand for the gauge integration can at most contain finite powers of $\kappa$, and therefore $h_{1}^{(f)}$ and $\bar{h}_{1}^{(f)}$. More precisely, for every flavor the maximal power one spatial point $\mathbf{n} \in V(G)$ can contribute at most $\left(h_{1}^{(f)}\right)^{2 N_{c}}$ and $\left(\bar{h}_{1}^{(f)}\right)^{2 N_{c}}$, corresponding to the degrees of freedom of one quark and antiquark for Wilson fermions.

If we want to apply the same arguments to the evaluation of the effective theory, we have to face the caveat mentioned at the end of the evaluation of the $\kappa^{2}$-action.

\subsubsection{Partial contractions}

As we have seen during the evaluation of the $\kappa^{2}$-action, evaluating the effective theory can correspond to neglecting some contractions when applying Wick's theorem to evaluate the Grassmann integrals in equation (4.219). That this can lead to problems can be illustrated in a simple example. Consider the $2 \times 2$-matrix

$$
Q=\left(\begin{array}{cc}
1+h & 1 \\
1 & 1+h
\end{array}\right)
$$


Its inverse reads

$$
Q^{-1}=\frac{1}{2 h+h^{2}}\left(\begin{array}{cc}
1+h & -1 \\
-1 & 1+h
\end{array}\right) .
$$

Now we solve a Grassmann integral involving this matrix using Wick's theorem:

$$
\begin{aligned}
\int \mathrm{d} \Psi_{1} \mathrm{~d} \bar{\Psi}_{1} \mathrm{~d} \Psi_{2} \mathrm{~d} \bar{\Psi}_{2} \bar{\Psi}_{1} \Psi_{1} \bar{\Psi}_{2} \Psi_{2} \exp (\bar{\Psi} Q \Psi) & =\operatorname{det}(Q)\left(Q_{11}^{-1} Q_{22}^{-1}-Q_{21}^{-1} Q_{12}^{-1}\right) \\
& =\operatorname{det}(Q) \operatorname{det}\left(Q^{-1}\right) \\
& =1
\end{aligned}
$$

Clearly, the result involves only finite powers of $h$. If one, however, considers only one half of the contractions, the result is

$$
-\operatorname{det}(Q) Q_{21}^{-1} Q_{12}^{-1}=-\frac{1}{2 h+h^{2}} .
$$

Obviously, this expression contains singularities which do not occur in the full result and a series expansion around $h=0$ will contain arbitrary powers of $h$. Those will only cancel when the other half of the contractions is included.

Another manifestation of this problem is that when equation (4.40) is used to rewrite equation (4.206), the result is

$$
\begin{aligned}
\left(\frac{1}{z_{0}}\right)^{3} \int \prod_{\mathbf{n} \in\left\{\mathbf{n}_{1}, \mathbf{n}_{2}, \mathbf{n}_{3}\right\}} \mathrm{d} W(\mathbf{n}) & \frac{1}{\operatorname{det}\left(q_{\text {stat }}\left(W\left(\mathbf{n}_{2}\right)\right)\right)} \\
& \times \int \prod_{\mathbf{n} \in\left\{\mathbf{n}_{1}, \mathbf{n}_{2}\right\}} \mathrm{d}[\Psi(\mathbf{n}), \bar{\Psi}(\mathbf{n})] e^{\Psi(\mathbf{n}) Q_{\text {stat }}(\mathbf{n}) \bar{\Psi}(\mathbf{n})} M_{s, 1}\left(\mathbf{n}_{1}\right), \\
& \times \int \prod_{\mathbf{n} \in\left\{\mathbf{n}_{2}, \mathbf{n}_{3}\right\}} \mathrm{d}[\Psi(\mathbf{n}), \bar{\Psi}(\mathbf{n})] e^{\Psi(\mathbf{n}) Q_{\text {stat }}(\mathbf{n}) \bar{\Psi}(\mathbf{n})} M_{s, 1}\left(\mathbf{n}_{2}\right)
\end{aligned}
$$

so in contrast to equation (4.219), the inverses of $\operatorname{det}\left(q_{\text {stat }}(W(\mathbf{n}))\right)$ do not cancel here explicitly. Now, to the orders considered here, all expressions are still well behaved, as one can show by doing the complete calculation. However, at higher orders the problem may get relevant. Certainly, considering the integral (4.119) and the discussion around it, the probability that invalid singularities and infinite powers of $h_{1}^{(f)}$ are generated is higher when the logarithmic resummation is not used. For analytic calculations, one can of course simply throw away higher orders in the final result, for simulations of the effective theory this is, however, not possible. Further investigations and higher order calculations are necessary to decide if the consequences of the problem can always be avoided. For this, it is useful to solve the occurring integrals without resorting to expansions in $h_{1}^{(f)}$, this can be achieved by using the angle parametrization that will be explained in section 6.1.1 and the residue theorem.

Next, we use the results obtained in this chapter (and which are free of the problem we just explained) to analyze some properties of the effective theory. 



\section{The onset transition for baryon and isospin chemical potential}

As a first test for our results for $N_{c}=3$, we will investigate for $N_{f}=2$ the onset transition for both baryon chemical potential

$$
\begin{aligned}
\mu_{B} & =\frac{3}{2} \mu^{(1)}+\frac{3}{2} \mu^{(2)} \\
& =\frac{3}{2} \mu_{u}+\frac{3}{2} \mu_{d}
\end{aligned}
$$

and isospin chemical potential

$$
\mu_{I}=\mu_{u}-\mu_{d} .
$$

The precise prefactors in front of the chemical potentials for up- and down-quarks are different depending on conventions, and the important part is the sign in front of them. For example the authors in [158] define

$$
\mu_{I}=\frac{\mu_{u}-\mu_{d}}{2}
$$

In our convention the transition to the pion condensed phase for $T=0$ happens when $\mu_{I}=m_{\pi}$.

The relations between these chemical potentials can be inverted to give

$$
\begin{aligned}
& \mu_{u}=\frac{\mu_{B}}{3}+\frac{\mu_{I}}{2}, \\
& \mu_{d}=\frac{\mu_{B}}{3}-\frac{\mu_{I}}{2} .
\end{aligned}
$$

We will assume the up- and down-quarks to be degenerate and since $N_{f}=2$ neglect all heavier quarks. The degeneracy of the quark masses implies that $\kappa_{u}=\kappa_{d}=\kappa$. Chemical potential enters the effective theory via $h_{u}=h_{1}^{(1)}, \bar{h}_{u}, h_{d}=h_{1}^{(1)}$ and $\bar{h}_{d}$. We consider the flavors separately here, in order to treat isospin and baryon chemical potential in a unified fashion.

While the baryon density indicates the overall surplus of strongly interacting matter, isospin density indicates the asymmetry between up and down quarks or, expressed in the effective degrees of freedom at lower energies, between protons and neutrons or positively charged pions over negatively charged pions. Typical systems with strong isospin asymmetry are non-central heavy-ion collision, where the initial state has around twice as many neutrons as protons, and cold neutron stars, where the contribution of protons is even lower [159]. Although nonzero $\mu_{I}$ therefore occurs in nature, systems which at the same time have $\mu_{B}=0$ do not occur and are, as noted in [160], unstable if one takes into account weak decays, which do not conserve isospin. Nevertheless, if one is mainly interested in QCD dynamics it is still interesting to study such a system. A big advantage when doing so is that it is in a nontrivial regime of QCD which has a positive and real fermion determinant [161]. Consequently, simulations using standard lattice Monte Carlo methods are possible. The pioneering works concerning these simulations were done in $[162,163]$ and for recent results we refer to $[158,164]$. Furthermore, the system is analytically tractable in several 
interesting limits, as the positivity of the fermion determinant allows for the derivation of QCD inequalities and one can use chiral perturbation theory for small $\mu_{I}$. This was first studied in [160, 165] and for more recent works we refer to [166]. Note that in these papers the authors work with negative $\mu_{I}$, which is, considering the systems mentioned above, more realistic. However, all our results are completely symmetric and we will work with $\mu_{I}>0$.

Although the sign problem for baryon chemical potential makes matters much more complicated for systems with $\mu_{B} \neq 0$, the onset transition at low temperatures can be understood in the continuum for both systems with pure baryon or isospin chemical potential, using simple statistical and phenomenological arguments. We will recount them here shortly, following roughly [167].

\subsection{Expectations}

First, we consider $\mu_{I}=0$ and $\mu_{B} \geq 0$ for $T=0$. Writing the grand-canonical partition function as a sum over all quantum states, which we denote by $\alpha$, one has

$$
Z=\sum_{\alpha} \exp \left(-\frac{E_{\alpha}-\mu_{B} N_{B, \alpha}}{T}\right)
$$

where $E_{\alpha}$ denotes the energy of the state $\alpha$ and $N_{B, \alpha}$ its baryon charge. In the limit $T \rightarrow 0$, the state which minimizes $E_{\alpha}-\mu_{B} N_{B, \alpha}$ dominates the partition function exponentially. For $\mu=0$ this state is simply the vacuum with (renormalized) energy $E_{0}=0$ and $N_{B, 0}=0$. When $\mu$ is increased, this does not change until it is possible to find a state with

$$
E_{\alpha}-\mu N_{\alpha} \leq 0 \Leftrightarrow \mu \geq \frac{E_{\alpha}}{N_{\alpha}}
$$

In other words, if we define

$$
\mu_{0}=\min _{\alpha}\left(\frac{E_{\alpha}}{N_{\alpha}}\right)
$$

the only relevant state contributing to the partition function for $\mu<\mu_{0}$ is still the vacuum. This means that at zero temperature the baryon number density has the property

$$
n_{B}(\mu)=0 \text { for } \mu<\mu_{0} .
$$

This phenomenon, that the thermodynamic functions stay independent of the chemical potential up to some threshold is called silver blaze phenomenon and has been demonstrated for isospin chemical potential explicitly from path integral considerations of QCD [168]. So far the whole argument here works completely analogously for the isospin case, the differences start when we want to find out the value for $\mu_{0}$.

If the Baryons were non-interacting, we would simply have that $\mu_{0}$ is the mass $m_{N}$ of the lightest nucleon of the system, i. e. the proton or neutron mass $m_{N}=m_{p}=m_{n}$. Being fermions, these particles can occupy every state only once and for increasing $\mu$ would gradually fill up states up to the at that point reachable Fermi energy. The number density would be continuous at $\mu=\mu_{0}$ and increase proportional to the volume of the Fermi sphere. Of course, in reality these nucleons are attractive and since binding energy corresponds to a negative energy contribution, $\mu_{0}$ is shift to the left of $m_{N}$ and it is energetically favorable to directly create several baryons at the threshold. More precisely, the energy per baryon $E / N$ can be equivalently written as $m_{N}-\left(N m_{N}-E\right) / N$. Consequently, the state which minimizes $E / N$ as demanded by equation (5.11) is that which maximizes the binding energy per nucleon $\epsilon_{b}=\left(N m_{n}-E\right) / N$. In pure QCD without electroweak interaction, the binding energy per nucleon in a nucleus increases with the number of nucleons and saturates at $\approx 16 \mathrm{MeV}$ [169]. Correspondingly, the baryon density jumps at $\mu_{0} \approx m_{N}-16 \mathrm{MeV}$ 
to the value of the density of nuclear matter, $n_{B, 0} \approx 0.16 \mathrm{fm}^{-3}$ and forms a macroscopically large sample of nuclear matter as defined by Fetter and Walecka [169] that is stabilized by Pauli repulsion. Therefore, one has a first-order phase transition characterized by a discontinuity in the function $n_{B}\left(\mu_{B}\right)$.

In contrast if we consider isospin chemical potential, the lightest particles are pions, which are bosons. In the non-interacting case, Bose-Einstein condensation would lead to a diverging isospin density at $\mu_{0}=m_{\pi}$. In QCD, the repulsive interaction of pions stabilizes the Bose-Einstein condensate. Although the repulsive interaction is a positive energy contribution, $\mu_{0}$ is not shift to the right in this case, as a single pion has nothing to interact with, and the energetically most favorable state at $\mu_{0}=m_{\pi}$ is exactly that state. Consequently, the isospin number density is continuous at $\mu=\mu_{0}$ and according to chiral perturbation theory it goes like [160]

$$
n_{I}=f_{\pi}^{2} \mu_{I}\left(1-\frac{m_{\pi}^{4}}{\mu_{I}^{4}}\right) .
$$

Chiral perturbation theory also predicts that the transition is of second order with the universality class $\mathrm{O}(2)$.

When the temperature is increased, the densities are non-zero for any $\mu>0$ and therefore cannot be used to predict phase transitions as for $T=0$. Nevertheless, on grounds of continuity, the transition in the case for Baryon chemical potential should stay first-order for sufficiently small $T$. Therefore, a line of first-order transitions emerges from $\mu_{0}$, as indicated in figure 2.1 (the slope of the line can be inferred from the Clausius-Clapeyron relations). Since there is no symmetry breaking order parameter there is no reason why the phases cannot be connected by a crossover. It is natural to expect that the first-order phase-transition line terminates in a second-order endpoint with the critical exponents of the 3-dimensional Ising model, as in a typical liquid-gas transition. As the binding energy is responsible for the transition being first order, it can be used to estimate the temperature of the endpoint to be of $\mathcal{O}(10 \mathrm{MeV})$.

In the isospin case, the pion condensate is a true order parameter indicating the pion condensed phase. Lattice calculations [158] show that the second order line $\mu_{I, c}(T)$ extends steeply to the zero-density deconfinement transition nearly parallel to the temperature axis, where it flattens out.

Certainly, we cannot reproduce these lattice results here with our calculation in the strong coupling limit to order $\mathcal{O}\left(\kappa^{4}\right)$. As we are dealing with a series expansion, we especially cannot reproduce phase transitions correctly. Consequently, we will content ourselves with a somewhat more modest approach, in which we will try to see if we are able to qualitatively reproduce the phenomenological properties that shape the transitions as we just described it. Promising results in this directions were obtain for baryon chemical potential in [65] to order $\kappa^{2}$. We will review these results here critically by extending them to $\kappa^{4}$ and isospin chemical potential.

To start off, we introduce how the observables we want to investigate can be obtained from our series of $\log (Z)$. In the following we work with $N_{c}=3$.

\subsection{Observables}

From equation (4.186) we obtain the free energy density in the thermodynamic limit

$$
-f=\lim _{N_{s} \rightarrow \infty} \frac{\log (Z)}{N_{s}} .
$$

All thermodynamic observables can be obtained by deriving this quantity. The hopping parameter $\kappa$ enters $f$ in two different ways: the first way is implicitly through the $h_{1}$ and $\bar{h}_{1}$ of the different flavors, corresponding to temporal hops, which are resummed. The second is explicitly through 
the spatial hops, and in this way is the parameter we expand around. In equation (2.51), this dependence is also implicit and is caught in all the effective couplings independent of chemical potential. Aiming for simplicity in our formulas we consider $f$ to depend explicitely on $\kappa$ due to spatial hops and an implicit dependence on $\kappa$ only occurs for temporal hops.

The pressure is obtained from

$$
a^{4} p=-\frac{f}{N_{\tau}}
$$

The quark number density for a specific flavor $q$ can be calculated using

$$
\begin{aligned}
a^{3} n_{q} & =a^{3} \frac{T}{V} \frac{\partial \log (Z)}{\partial \mu_{q}} \\
& =-\frac{\partial f}{\partial h_{q}} h_{q}+\frac{\partial f}{\partial \bar{h}_{q}} \bar{h}_{q} .
\end{aligned}
$$

The following observation is rather trivial but relevant when discussing the binding energy of the particles in the system: The quark number density does not correspond to the actual number of quarks per unit volume in the system, but to the difference of the number of quarks and antiquarks of that flavor (which is the conserved charge associated with the $U(1)$-flavor symmetry of the QCD-Lagrangian). To distinguish this in our notation we will write

$$
n_{q}=\tilde{n}_{q}-\tilde{n}_{\bar{q}}
$$

In similar fashion, the baryon number and isospin densities are computed from

$$
\begin{aligned}
a^{3} n_{B} & =a^{3} \frac{T}{V} \frac{\partial \log (Z)}{\partial \mu_{B}} \\
& =-\frac{1}{3}\left(\frac{\partial f}{\partial h_{u}} h_{u}-\frac{\partial f}{\partial \bar{h}_{u}} \bar{h}_{u}+\frac{\partial f}{\partial h_{d}} h_{d}-\frac{\partial f}{\partial \bar{h}_{d}} \bar{h}_{d}\right) \\
& =a^{3} \frac{\left(n_{u}+n_{d}\right)}{3}, \\
a^{3} n_{I} & =a^{3} \frac{T}{V} \frac{\partial \log (Z)}{\partial \mu_{I}} \\
& =-\frac{1}{2}\left(\frac{\partial f}{\partial h_{u}} h_{u}-\frac{\partial f}{\partial \bar{h}_{u}} \bar{h}_{u}-\frac{\partial f}{\partial h_{d}} h_{d}+\frac{\partial f}{\partial \bar{h}_{d}} \bar{h}_{d}\right) \\
& =a^{3} \frac{\left(n_{u}-n_{d}\right)}{2} .
\end{aligned}
$$

The energy density is obtain by a derivative with respect to the inverse temperature at fixed fugacity. Using the fact that $f$ is volume independent, we turn this into a derivative with respect to the lattice spacing

$$
\begin{aligned}
a^{3} e & =-\left.a^{3} \frac{1}{V} \frac{\partial \log (Z)}{\partial(1 / T)}\right|_{z}, \\
& =\frac{1}{N_{\tau}}\left(\left.\frac{\partial f}{\partial h_{u}} \frac{\partial h_{u}}{\partial a}\right|_{z}+\left.\frac{\partial f}{\partial \bar{h}_{u}} \frac{\partial \bar{h}_{u}}{\partial a}\right|_{z}+\left.\frac{\partial f}{\partial h_{d}} \frac{\partial h_{d}}{\partial a}\right|_{z}+\left.\frac{\partial f}{\partial \bar{h}_{d}} \frac{\partial \bar{h}_{d}}{\partial a}\right|_{z}+\frac{\partial f}{\partial \kappa} \frac{\partial \kappa}{\partial a}\right) \\
& =\frac{1}{\kappa} \frac{\partial \kappa}{\partial a}\left(\frac{\partial f}{\partial h_{u}} h_{u}+\frac{\partial f}{\partial \bar{h}_{u}} N_{\tau} \bar{h}_{u}+\frac{\partial f}{\partial h_{d}} N_{\tau} h_{d}+\frac{\partial f}{\partial \bar{h}_{d}} N_{\tau} \bar{h}_{d}+\frac{\partial f}{\partial \kappa} \frac{\kappa}{N_{\tau}}\right) \\
& =-\frac{1}{\kappa} \frac{\partial \kappa}{\partial a}\left(\tilde{n}_{u}+\tilde{n}_{\bar{u}}+\tilde{n}_{d}+\tilde{n}_{\bar{d}}-\frac{\partial f}{\partial \kappa} \frac{\kappa}{N_{\tau}}\right) .
\end{aligned}
$$


To compute the derivative of $\kappa$ with respect to the lattice distance we use the expression for the pion mass in the strong coupling limit from [170] and, to be consistent in our expansion, expand it up to $\mathcal{O}\left(\kappa^{4}\right)$

$$
m_{\pi}=\frac{1}{a}\left(2 m-6 \kappa^{2}-54 \kappa^{4}+\mathcal{O}\left(\kappa^{6}\right)\right),
$$

where $a m=-\log (2 \kappa)$ corresponds to the constituent quark mass to leading order. Demanding that the pion mass stays constant when $a$ is adjusted implicitly defines $\kappa$ as a function of $a$ and its derivative can be computed from

$$
0=\frac{\mathrm{d} m_{\pi}}{\mathrm{d} a}=\frac{\partial m_{\pi}}{\partial a}+\frac{\partial m_{\pi}}{\partial \kappa} \frac{\partial \kappa}{\partial a}
$$

which implies

$$
\begin{aligned}
a \frac{\partial \kappa}{\partial a} & =-a \frac{\frac{\partial m_{\pi}}{\partial a}}{\frac{\partial m_{\pi}}{\partial \kappa}} \\
& =\kappa \frac{\log (2 \kappa)+3 \kappa^{2}+27 \kappa^{4}+\mathcal{O}\left(\kappa^{6}\right)}{1+6 \kappa^{2}+108 \kappa^{4}+\mathcal{O}\left(\kappa^{6}\right)} \\
& =-\kappa\left(a m-(3+2 m) \kappa^{2}-(9+8 m) \kappa^{4}+\mathcal{O}\left(\kappa^{6}\right)\right) .
\end{aligned}
$$

It will be useful to get an estimate for the binding energy between the particles in the system. In previous studies [65] with baryon chemical potential only, the following quantity was used at low temperatures, where thermal fluctuation are assumed to be suppressed:

$$
\epsilon=\frac{e-n_{B} m_{B}}{n_{B} m_{B}}
$$

where $m_{B}$ is the baryon mass, which we also take from [170] to $\mathcal{O}\left(\kappa^{4}\right)$

$$
m_{B}=\frac{1}{a}\left(3 m-6 \kappa^{3}+\mathcal{O}\left(\kappa^{3}\right)\right) .
$$

In [74] the situation was analyzed in more detail: in the cold and dense case with baryon chemical potential one can make the approximation $\bar{h}_{1}^{(u)}=\bar{h}_{1}^{(d)}=0$ and then the energy density reads

$$
a^{3} e=-\frac{1}{\kappa} \frac{\partial \kappa}{\partial a}\left(3 n_{B}-\frac{\partial f}{\partial \kappa} \frac{\kappa}{N_{\tau}}\right) .
$$

To leading order one has $-\frac{\partial \kappa}{\partial a} / \kappa=m$, suggesting the definition

$$
m_{\mathrm{eff}}=-\frac{1}{\kappa} \frac{\partial \kappa}{\partial a}
$$

It was concluded that the part of the energy density containing the baryon number density is related to the rest energy and the quantity estimating the binding energy was defined to be: ${ }^{1}$

$$
\epsilon=\frac{e-3 m_{\mathrm{eff}} n_{B}}{3 m_{\mathrm{eff}} n_{B}}
$$

Now, if we want to discuss baryon and isospin chemical potential on equal footing, we should also discuss what happens when the the $\bar{h}_{1}$ are not neglected. In general we note that in the baryon and

\footnotetext{
${ }^{1}$ In [65], to compute this derivative of $\kappa$ it was argued that at leading order $\frac{\partial \kappa}{\partial a}=-\kappa \frac{m_{B}}{3}$, making the approaches compatible: in both cases the part of $e$ that contains the baryon number density is subtracted
} 
isospin density equations (5.20) and (5.23) the quark and antiquark contributions have opposite sign, while they both have a positive contribution in the energy density equation (5.28), which of course makes intuitive sense. Therefore, we estimate the binding energy using the quantity

$$
\epsilon=\frac{e-m_{\mathrm{eff}}\left(\tilde{n}_{u}+\tilde{n}_{\bar{u}}+\tilde{n}_{d}+\tilde{n}_{\bar{d}}\right)}{m_{\mathrm{eff}}\left(\tilde{n}_{u}+\tilde{n}_{\bar{u}}+\tilde{n}_{d}+\tilde{n}_{\bar{d}}\right)}
$$

\subsection{Static case}

The leading order expression in the free energy density due to $\log \left(z_{0}\right)$ contains contributions from temporal quark hops only and therefore is called the static case. The partition function corresponds to the $N_{s}$-fold product of the partition function $z_{0}$ for $0+1$ dimensional QCD, which means it represents a non-interacting system. This case has been discussed in [65] already, but it will be useful to recount the observations made there and add some additional remarks.

The expressions for $z_{0}$ for the general $N_{f}=2$ case already has 801 terms, so it would be very cumbersome to discuss. Some simplifications occur however when one considers very small temperatures (large $N_{\tau}$ ) and either exclusively baryon chemical potential or only isospin chemical potential. In the first case one can set $\bar{h}_{u / d}=0$ and in the second case $\bar{h}_{u}=h_{d}=0$. The partition function for the first case then reads

$$
Z=\left(z_{0}\right)^{N_{s}}
$$

with

$$
\begin{aligned}
z_{0}= & 1+4 h_{d}^{3}+h_{d}^{6}+6 h_{d}^{2} h_{u}+4 h_{d}^{5} h_{u}+6 h_{d} h_{u}^{2}+10 h_{d}^{4} h_{u}^{2}+4 h_{u}^{3}+20 h_{d}^{3} h_{u}^{3}+4 h_{d}^{6} h_{u}^{3}+10 h_{d}^{2} h_{u}^{4} \\
& +6 h_{d}^{5} h_{u}^{4}+4 h_{d} h_{u}^{5}+6 h_{d}^{4} h_{u}^{5}+h_{u}^{6}+4 h_{d}^{3} h_{u}^{6}+h_{d}^{6} h_{u}^{6},
\end{aligned}
$$

while for the second case one has

$$
\begin{aligned}
z_{0}= & 1+4 \bar{h}_{d}^{3}+\bar{h}_{d}^{6}+4 \bar{h}_{d} h_{u}+6 \bar{h}_{d}^{4} h_{u}+10 \bar{h}_{d}^{2} h_{u}^{2}+6 \bar{h}_{d}^{5} h_{u}^{2}+4 h_{u}^{3}+20 \bar{h}_{d}^{3} h_{u}^{3}+4 \bar{h}_{d}^{6} h_{u}^{3}+6 \bar{h}_{d} h_{u}^{4} \\
& +10 \bar{h}_{d}^{4} h_{u}^{4}+6 \bar{h}_{d}^{2} h_{u}^{5}+4 \bar{h}_{d}^{5} h_{u}^{5}+h_{u}^{6}+4 \bar{h}_{d}^{3} h_{u}^{6}+\bar{h}_{d}^{6} h_{u}^{6} .
\end{aligned}
$$

The sums of the exponents in $h_{u}^{n} h_{d}^{m}$ in the case with baryon chemical potential are multiples of three, $n+m=0 \bmod 3$. The partition function has the form of a free baryon gas [171] and the prefactors give the degeneracy of the spin multiplets, for example $h_{d}^{3}$ corresponds to the $\Delta^{-}$ quadruplet. All in all, equation (5.41) contains all baryonic spin-flavor multiplets that are consistent with the Pauli principle, the fact that quarks are spin $1 / 2$ fermions, the number of flavors in the system and the gauge group. The isospin case equation (5.42) follows the same logic, only now one also has anti-baryons and mesons. In both cases, the quark chemical potentials can be characterized by a single chemical potential and we define in the case with only baryon chemical potential $\mu:=\mu_{u}=\mu_{d}=\mu_{B} / 3$ and in the isospin case $\mu:=\mu_{u}=-\mu_{d}=\mu_{I} / 2$. If we now consider the limit $T \rightarrow 0\left(N_{\tau} \rightarrow \infty\right)$ and use that $h_{1}$ for the flavor $q$ can be written as $h_{q}=\exp \left(\left(\mu_{q}-m\right) / T\right)$ one has for both cases that

$$
\begin{aligned}
& \lim _{T \rightarrow 0} a^{4} p= \begin{cases}0 & \text { if } \mu<m, \\
2 N_{f} N_{c}(a \mu-a m) & \text { if } \mu>m,\end{cases} \\
& \lim _{T \rightarrow 0} a^{3} n= \begin{cases}0 & \text { if } \mu<m, \\
2 N_{f} N_{c} & \text { if } \mu>m,\end{cases}
\end{aligned}
$$

where $n=3 n_{B}$ in the first case and $n=2 n_{I}$ in the second case. The isospin density is zero in the first case and the same is true for $n_{B}$ in the second case. We note that the thermodynamic 

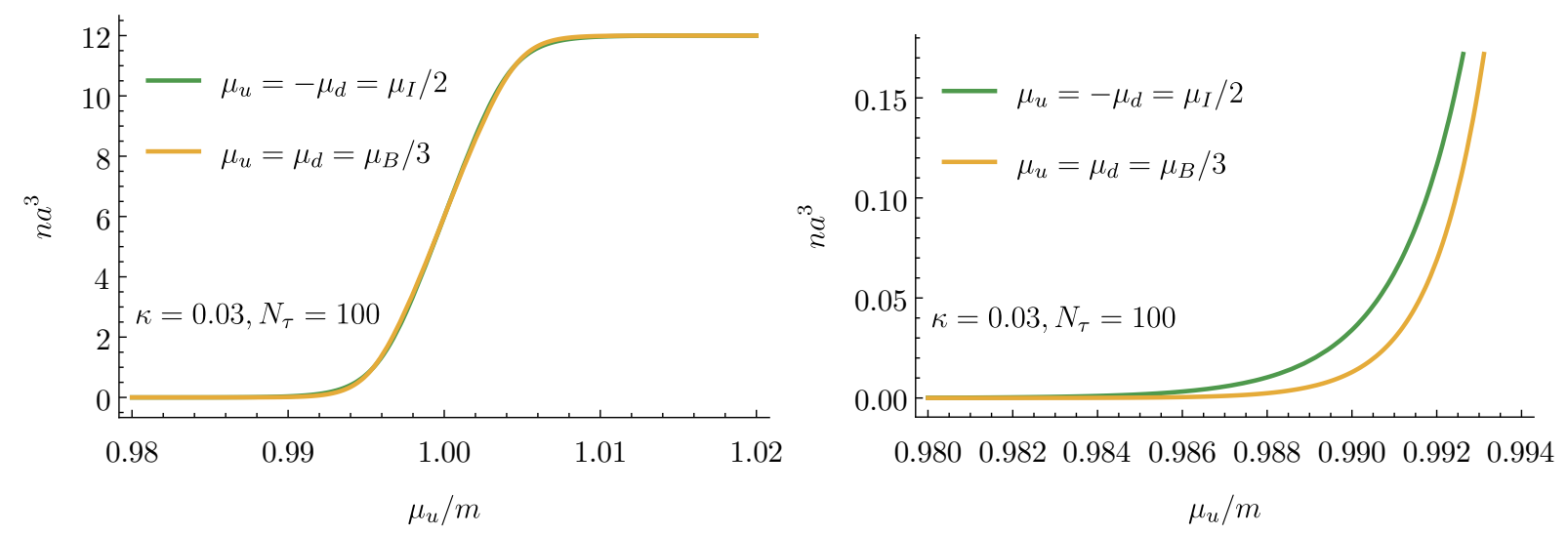

Figure 5.1: Comparison of baryon and isospin chemical potential and their associated densities in the static approximation. On the y-axis, $n=3 n_{B}$ in the case where $\mu_{I}=0$ (yellow line) and $n=2 n_{I}$ for the case $\mu_{B}=0$ (green line)

functions stay zero (and therefore independent of the chemical potential) in both cases up until the chemical potential for the quarks is larger than the constituent quark mass. Therefore we observe here the silver blaze property. Furthermore, since all states in the system have masses which are simply multiples of the same constituent quark mass, which means, for example that $m_{N} / 3=m_{\pi} / 2$, it is in the $T \rightarrow 0$ limit energetically most convenient for the system to produce those particles which have the largest number of constituents, as soon as $\mu>m$. In both cases this is a bosonic state, $h_{u}^{6} h_{d}^{6}$ in the first case and $h_{u}^{6} \bar{h}_{d}^{6}$ in the second, which can condense according to Bose-Einstein statistics. This state however is a composite state of fermionic particles, so as soon as all possible quark states per lattice site are occupied, the number density reaches saturation at $2 N_{f} N_{c}$ due to the Pauli principle of the constituent quarks. Therefore, the saturation density is not that of nuclear matter, but instead it is determined by the degrees of freedom of the quarks.

The resulting step function is smeared out as soon as $T>0$ is considered, which is illustrated in figure 5.1. In this figure we also note that the densities for both cases are very similar. Taking a closer look around the region where the densities are starting to rise, one notices that the density for the case of isospin chemical potential starts to rise for lower $\mu$. This is due to the fact that although $m_{N} / 3=m_{\pi} / 2$ one has of course $m_{N}>m_{\pi}$ and the latter inequality is relevant when considering the creation of particles due to thermal excitations. In other words, while for $T=0$ the constituent quark mass alone decides the position of the onset, for $T>0$ the total mass (i.e. the sum of the constituent quark masses) is also relevant. It is important to note this, otherwise one can confuse thermal effects with mass corrections which arise when corrections to the static case are considered.

\subsection{Spatial $\kappa$-corrections}

Going beyond the static approximation we add $\kappa^{2}$-corrections from spatial hoppings and start to resolve interactions between the baryons and mesons. Being perturbative in nature, one is not able to obtain a true phase transition of the $3+1$ dimensional theory as described in section 5.1 and in the limit $N_{\tau} \rightarrow \infty$ the density reduces to equation (5.44), i. e. the static result, to the orders considered here (up to $\mathcal{O}\left(\kappa^{4}\right)$, but we do not expect this to change when including higher orders, except of cases where additional resummations have been obtained). Furthermore, considering our experiences from the spin model, we certainly do not have enough orders to consider estimates of convergence via, for example, the ratio method or Padé approximants. 

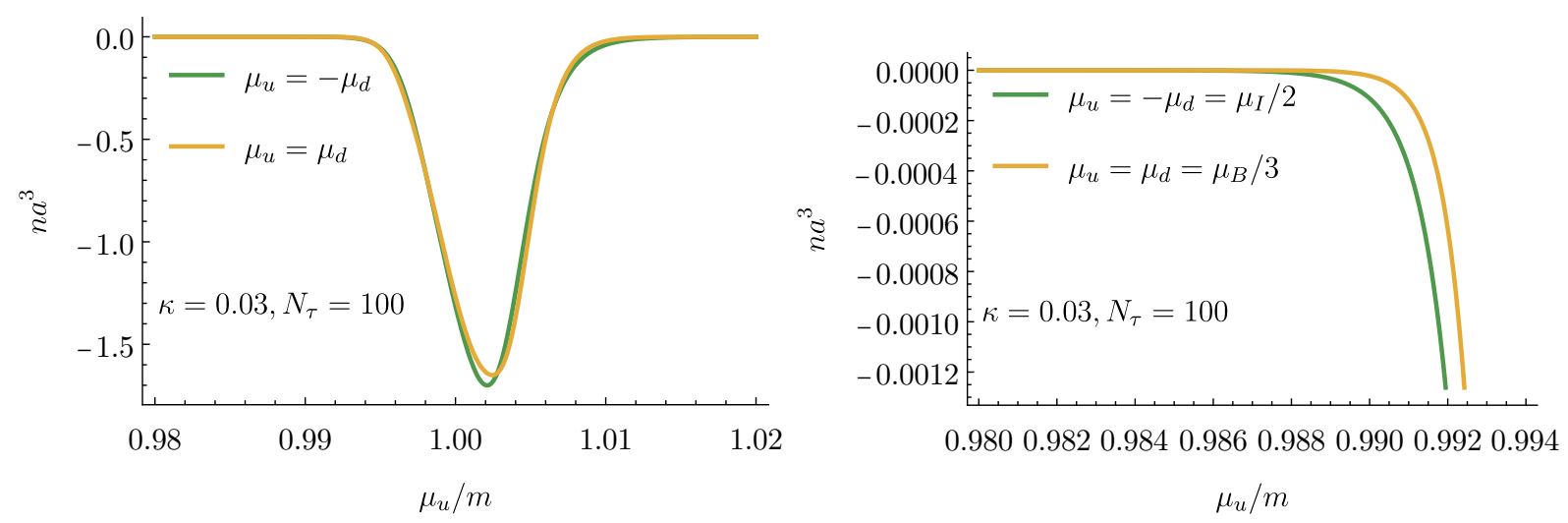

Figure 5.2: Comparison of baryon and isospin chemical potential and the $\kappa^{2}$ contribution of their associated densities. The meaning of the y-axis is the same as in figure 5.1. Note that the chemical potential is still normalized by the leading order constituent quark mass.

Therefore, as we mention above already, we content ourselves with an investigation of qualitative properties which lead to the picture outlined in section $5.1 \mathrm{In}$ [65] $\kappa^{2}$-corrections were considered in the case $N_{f}=1$ and it was shown that to these orders the quantity $\epsilon$ is negative, leading to the conclusion that one "can unambiguously identify this transition as baryon condensation". The same observation should be true (and has been made in [74]) for $N_{f}=2$ when baryon chemical potential is used exclusively. Indeed, neglecting $\bar{h}_{u / d}$ as in the previous section and introducing $h=h_{u}=h_{d}$ since $\mu_{u}=\mu_{d}$ in the case of baryon chemical potential, one obtains

$$
\epsilon=-\frac{4 h\left(3 h^{11}+45 h^{8}+75 h^{5}+15 h^{2}\right)^{2}}{\left(6 h^{11}+90 h^{8}+150 h^{5}+30 h^{2}\right)\left(h^{12}+20 h^{9}+50 h^{6}+20 h^{3}+1\right)} \kappa^{2} .
$$

This quantity is always negative and its modulus gets smaller when $\kappa$ decreases, implying weaker binding energy between the baryons for heavier quarks. As a result, the first order region for the liquid gas transition gets pushed to lower temperatures when the quark mass is increased, just like it was concluded for $N_{f}=1$ in [65].

Considering the case with isospin chemical potential makes it clear that the situation is a bit more complicated. Neglecting $\bar{h}_{u}$ and $h_{d}$ and writing $h=h_{u}=\bar{h}_{d}$ one obtains

$$
\begin{aligned}
\epsilon= & -\frac{4 h\left(3 h^{11}+10 h^{9}+18 h^{8}+20 h^{7}+21 h^{6}+33 h^{5}+15 h^{4}+10 h^{3}+6 h^{2}+2 h\right)^{2}}{\left(6 h^{11}+20 h^{9}+36 h^{8}+40 h^{7}+42 h^{6}+66 h^{5}+30 h^{4}+20 h^{3}+12 h^{2}+4 h\right)} \\
& \times \frac{1}{\left(h^{12}+4 h^{10}+8 h^{9}+10 h^{8}+12 h^{7}+22 h^{6}+12 h^{5}+10 h^{4}+8 h^{3}+4 h^{2}+1\right)} \kappa^{2} .
\end{aligned}
$$

Notably, $\epsilon$ is also always negative, just like in the case with baryon chemical potential. This goes against the expectation of repulsive pions condensing in the system. Another indication that we cannot observe some important differences between nucleons and pions can be noted by looking at the $\kappa^{2}$-corrections to the densities. Note that to be within a reasonable range of convergence for our expansion (which only goes to $\kappa^{4}$ ), the corrections to the static determinant should not change the results from the static case significantly. Therefore, to get a clear look at these corrections we will sometimes plot them alone and not the result to that order. The $\kappa^{2}$-correction to the density is shown in figure 5.2. Clearly, the gap between the densities when they are starting to rise does not significantly change when $\kappa^{2}$ corrections are considered, going against the expectation that in reality $m_{N} / 3>m_{\pi} / 2$. Of course binding energy has to be taken into account for the densities and if indeed the pions were repulsive and the nucleons attractive, then the baryon onset gets shifted 

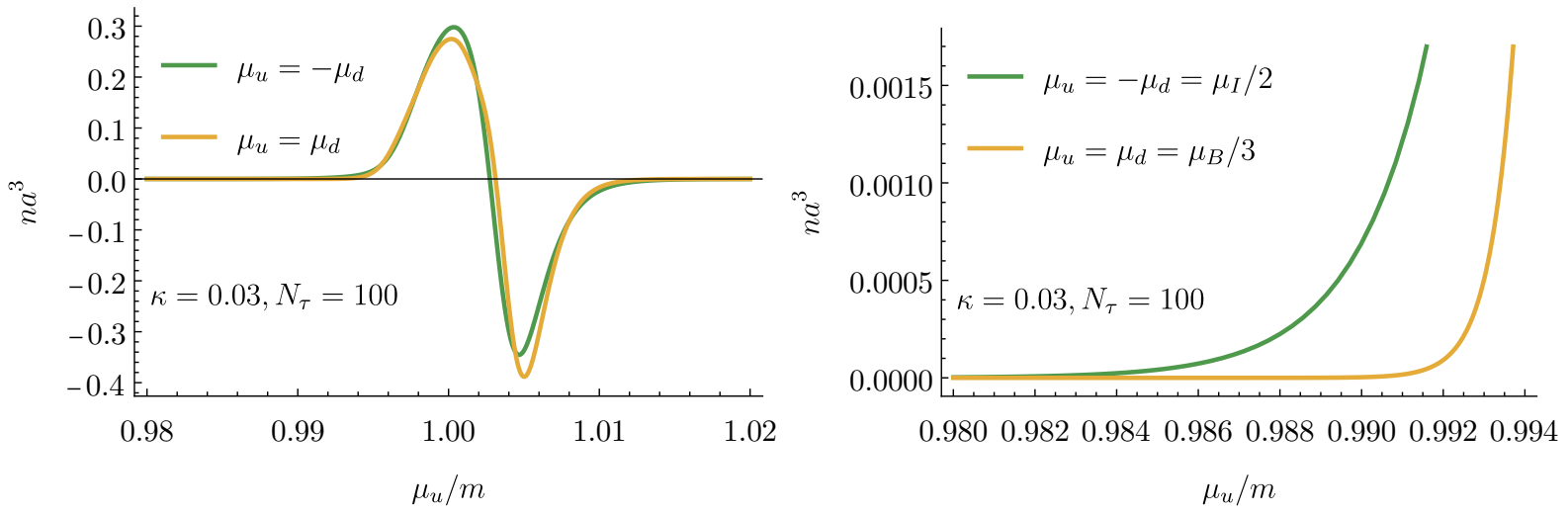

Figure 5.3: Same as figure 5.2, but for the $\kappa^{4}$-corrections.
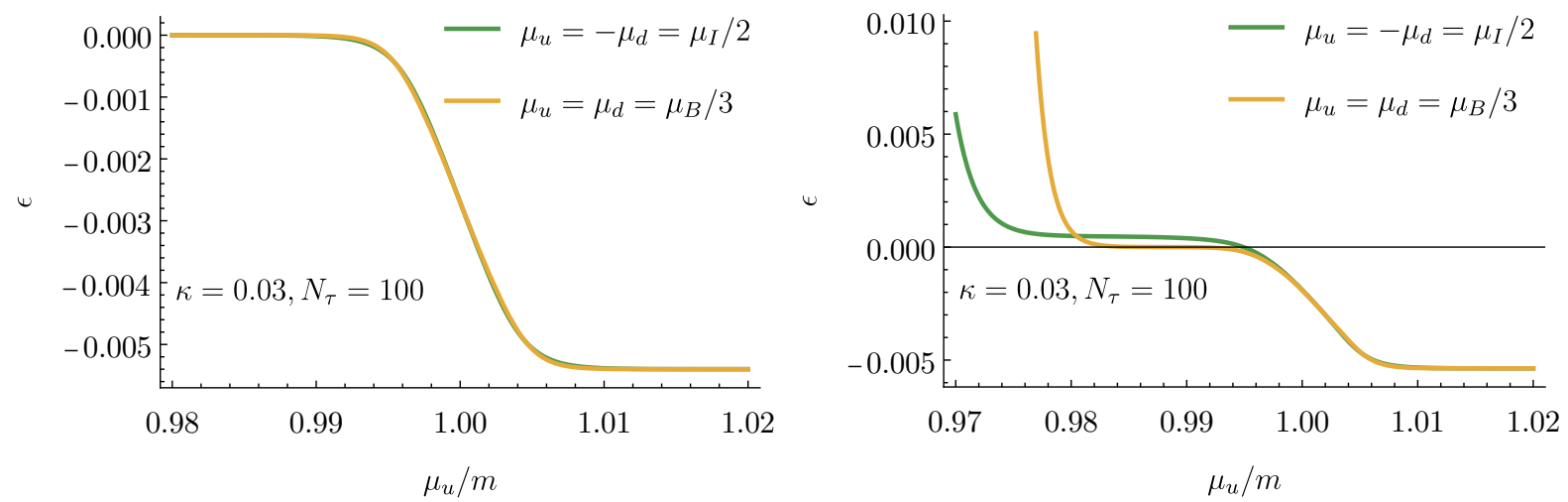

Figure 5.4: Comparison of $\epsilon$ for baryon and isospin chemical potential to $\mathcal{O}\left(\kappa^{2}\right)$ (left) and $\mathcal{O}\left(\kappa^{4}\right)$ (right).

left due to the binding energy and could potentially cancel the occurring difference due to the masses. But as we have seen, this cannot be the case here.

The situation improves when the $\kappa^{4}$-corrections are considered. Since the terms become rather complicated and consist of several terms with differing signs in this case, it is more convenient to look at plots of the situation. First, figure 5.3 shows the densities at that order, where a wider gap between the isospin and baryon densities becomes visible. Concerning the binding energy, figure 5.4 compares the results for $\epsilon$ to $\mathcal{O}\left(\kappa^{2}\right)$ and $\mathcal{O}\left(\kappa^{4}\right)$. The $\mathcal{O}\left(\kappa^{2}\right)$ results are to be expected from equations (5.45) and (5.46). For the next order, two interesting observations can be made: first, both for baryon and isospin chemical potential, $\epsilon$ diverges when small chemical potential is approached. This has to do with the fact that the definition of $\epsilon$ in equation (5.39) consists of a denominator that goes to zero for vanishing densities. Therefore, $\epsilon$ becomes very sensitive to thermal fluctuations for smaller densities. The second interesting observation is that roughly for $0.982 \lesssim \mu_{u} / m \lesssim 0.992, \epsilon$ drops to zero for baryon chemical potential, while this is not the case for isospin chemical potential, a possible indication for repulsive pions. Since the vanishing denominator in $\epsilon$ makes it hard to judge this effect, we also plot the numerator of $\epsilon$ in figure 5.5. In this case there is a clear positive contribution at $\mathcal{O}\left(\kappa^{4}\right)$, that may be attributed to repulsive pions. The fact that this quantity becomes negative for larger isospin chemical potential can be understood from the fact that isospin chemical potential can be also used to create baryons like the neutron, which furthermore have very similar constituent quark masses compared to the pions for the orders we consider here. In any case, the positive "hump" observed in the numerator of $\epsilon$ can only be considered as an indicator for a trend, as it only occur at the highest order we considered 

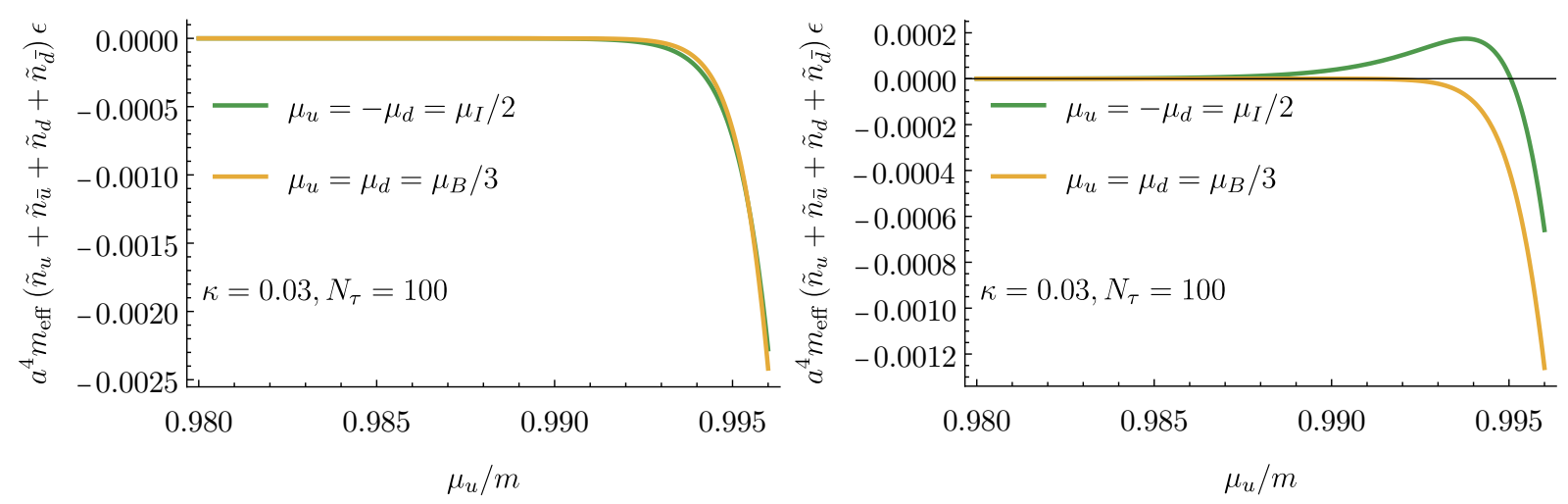

Figure 5.5: Comparison of the denominator of $\epsilon$ for baryon and isospin chemical potential to $\mathcal{O}\left(\kappa^{2}\right)$ (left) and $\mathcal{O}\left(\kappa^{4}\right)$ (right).

and it is therefore an effect which is outside of convergence and not under control.

The $\kappa^{4}$-action (including gauge corrections) has also been simulated [65], and in this case it was possible to observe a wider gap between baryon and isospin chemical potential, for larger $\kappa$ than we can consider here due to a lack of convergence. This is a good illustration of the fact that the evaluation of the effective theory leads to a partial resummation of some contributions in comparison to a direct evaluation, although it might be interesting to investigate these simulations closer with the insights of section 4.4 in mind. Also in simulations, it is possible to observe phase transitions of the effective theory and a first order liquid gas transition with a critical endpoint has been observed for the case with baryon chemical potential. It would be interesting to see if a second order line can be observed in the effective theory with isospin chemical potential. Additionally, this question can also be investigated using mean field methods, which have been applied to the $N_{f}=1$ case (without logarithmic resummations) in [172].

All in all, it is hard to make conclusive statements at the low orders we considered here, but we can extract more information from the coefficients for the thermodynamic observables we obtained so far by generalizing them to arbitrary $N_{c}$. This is because then some general conjectures concerning the scaling of all coefficients with respect to $N_{c}$ can be made. 


\section{Large $N_{c}$ behavior of the effective theory, quarkyonic matter}

Except of the discussion in section 4.6.3 and chapter 5, our approach was generic for $N_{c} \geq 3$. In contrast to the previous chapter, we will now set $\mu_{I}=0$ and consider baryon chemical for general $N_{c} \geq 3$

$$
\mu_{B}=N_{c} \mu,
$$

where $\mu$ now denotes the quark chemical potential (we will assume that all flavors are degenerate). We will show how to generalize the relevant temporal gauge integrals for baryon chemical potential up to $\kappa^{4}$ in the next section. Being able to vary $N_{c}$ freely, it is interesting to see what happens, when $N_{c}$ is made large. Specifically, we want to investigate the large $N_{c}$ considerations that lead to the prediction of quarkyonic matter in [173].

The large $N_{c}$ limit, when defined appropriately, has many interesting simplifications for continuum QCD. This can give ideas for intuitive explanations of many properties of real word QCD. We will not be able to give an exhaustive list of all the relevant work that has appeared in the more than four decades after the limit had been introduced by 't Hooft [174]. For a nice introduction including discussions of lattice calculations we refer to [175]. Lattice calculations are mainly concerned with vacuum properties or finite $T$ with $\mu_{B}=0$ (for obvious reasons), for an overview of recent works see [176] and references therein, but we want to discuss what happens when baryon chemical potential $\mu_{B}$ is considered.

We note that in the continuum, baryon matter has been treated in the combined heavy quark and large $N_{c}$ limits by a mean field analysis $[177,178]$, but our approach here, based on heavy but finite quark masses, series expansion and not only large but also general $N_{c}$, is quite different. The mean field approach has also been applied to the large $N_{c}$ limit of the spin-model of chapter 3 , where it is believed to be exact [179]. This work has recently been critically examined in [180], where the difference between $\mathrm{U}\left(N_{c}\right)$ and $\mathrm{SU}\left(N_{c}\right)$ was stressed (just like we will do below and already did in [93]), and it was suggested that the analysis should be repeated for the $\mathrm{SU}\left(N_{c}\right)$ model. When this is achieved, it should be interesting to see if the approach can be applied to the resummed Polyakov loop actions we are using.

Let us describe shortly the main features of QCD in the limit. In his pioneering work, 't Hooft noticed that the limit $N_{c} \rightarrow \infty$ is well-defined provided that at the same time the coupling $g \rightarrow 0$ such that $\lambda_{H}:=g^{2} N_{c}$ is kept fixed. In doing so, the number of flavors $N_{f}$ is also kept fixed. There is and alternative approach to take $N_{c} \rightarrow \infty$ where $N_{f} / N_{c}$ is kept fixed [181], but we will not discuss it here. In the 't Hoof limit, it turns out that non-planar Feynman diagrams are suppressed by $N_{c}^{-2}$ and quark loops in Feynman diagrams are suppressed by $N_{c}^{-1}$. This leads to a selection rule for Feynman diagrams for $N_{c} \rightarrow \infty$. Although the initial hope of being able to sum all the non-negligible diagrams in the limit is far from being achieved so far, interesting insights into the structure of QCD in this limit can be gained. Assuming that QCD is confining in the 't Hoof limit, it is concluded that the lightest physical states in the spectrum are glueballs and mesons with masses $\sim \Lambda_{\mathrm{QCD}}$. Furthermore, they are stable and non-interacting with interactions suppressed at least by $N_{c}^{-1 / 2}$.

Baryons are harder to treat diagrammatically. Consisting of $N_{c}$ valence quarks even their operator structure depends explicitly on $N_{c}$. Using Hamiltonian and path integral methods, Witten 

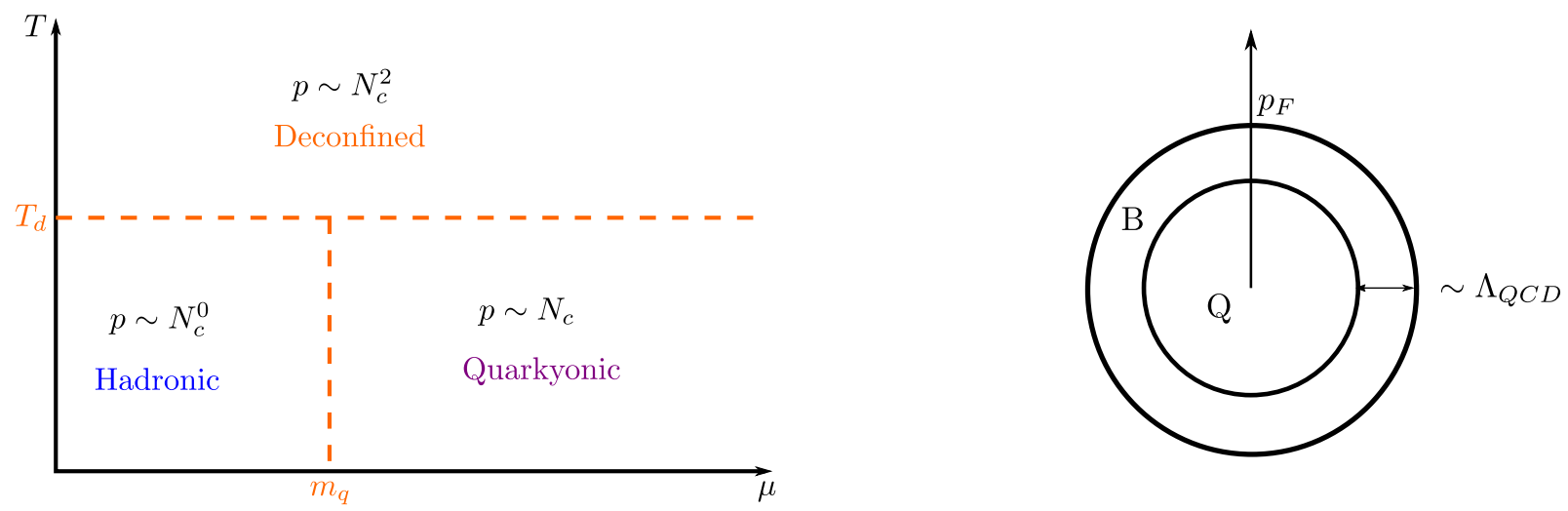

Figure 6.1: Taken from [185]. Left: conjectured phase diagram in the limit of large $N_{c}$ according to [173]. Right: momentum space illustration of quarkyonic matter, with quark matter (Q) inside the Fermi sea, surrounded by a Baryon shell (B) of width $\sim \Lambda_{\mathrm{QCD}}$

[182] showed that the baryon masses are $\sim N_{c} \Lambda_{\mathrm{QCD}}$ and baryons are strongly interacting with interactions $\sim N_{c}$.

In [173], the $(T, \mu)$-phase diagram was explored in the large $N_{c}$ limit. We give a short summary of some of the ideas of that paper. For pedagogical introductions to the ideas presented in that paper, see [183, 184].

Note that although one has baryon chemical potential, the quark chemical potential $\mu$ is the finite quantity. The phase diagram is divided into three phases, that are characterized by the $N_{c}$ scaling of the pressure, see figure 6.1

The hadronic phase, which can be well described by a hadron resonance gas [186, 187], consists of non-interacting mesons and gluestates and is distinguished by vanishing baryon number, which is suppressed exponentially as $N_{c} \rightarrow \infty$ since the baryons are so massive in this limit. Consequently the pressure in this phase has the scaling behavior $p \sim N_{c}^{0}$.

As quark loops are suppressed, the deconfinement transition is determined by gluon loops alone and becomes equivalent to the pure Yang-Mills transition and independent of chemical potential in the large $N_{c}$ limit. Yang-Mills theory is known to have a first order transition at $N_{c}=3$ and lattice simulations $[188,189]$ indicate that the first order transition persists at large $N_{c}$ with latent heat growing as $N_{c}^{2}$. Consequently, the deconfinement transition forms a first-order line parallel to the chemical potential axis. In the perturbative regime, the pressure is dominated by gluon degrees of freedom and since the gluons are in the adjoint representation of the gauge group it goes like $p \sim N_{c}^{2}$.

When $\mu_{B}>m_{B}$ (strictly speaking this happens earlier due to the binding energy, see section 5.1), the baryon number is non-vanishing also for temperatures below the deconfinement transition. For dense baryons, since the interactions between any number of baryons are $\sim N_{c}$, all baryon interactions contribute equally to give a pressure $p \sim N_{c}$. On the other hand, when $\mu \gg \Lambda_{\mathrm{QCD}}$ (but not necessarily $\mu / \Lambda_{\mathrm{QCD}}$ as large as some power of $N_{c}$ ) the pressure can be reliably determined using perturbation theory and one also predicts $p \sim N_{c}$. To understand how this scaling can consistently arise from strongly interacting baryons as well as perturbative calculations where quark-like excitations give the main contribution, one examines the case $\mu \gg \Lambda_{\mathrm{QCD}}$ closer. In momentum space, deep inside the (highly non-ideal) Fermi sea, the quarks interact at short distances and can be described perturbatively. However, even at large quark chemical potential, one has to consider the scattering of particles within $\sim \Lambda_{\mathrm{QCD}}$ of the Fermi surface separately (even though their contribution might be numerically small to the pressure). These particles interact by exchanging gluons with momenta $\sim \Lambda_{\mathrm{QCD}}$, and since quark loops are suppressed at large $N_{c}$, they cannot screen these 
gluon no matter how large the chemical potential is (unless the quark chemical potential itself scales like some power of $N_{c}$ ). Therefore these particles are strongly interacting and the scattering should be considered as that of baryons. This leads to the suggestion that this phase is described by "quarkyonic" matter, which in momentum space can be described as a quark sea with a baryonic Fermi surface, see figure 6.1. The width of this surface is $\Lambda_{\mathrm{QCD}}$ and this picture can also describe the situation where one only has dense baryons: for $\mu \sim \Lambda_{\mathrm{QCD}}$ the baryon surface is the whole sea. As $\mu$ increases one interpolates smoothly between a quarkyonic phase with a baryon surface that is wide (relative to $\mu$ ) to one where the baryon surface is narrow in relation to $\mu$. There are speculations of signatures of quarkyonic physics in heavy-ion collisions [190] and neutron stars [191].

We will check now the effective theory for the scaling properties that lead to the prediction of quarkyonic matter by direct calculation. The results we obtain in this way have been published in [185] and we will give here some more details for the calculations and correct some minor errors, which do not change the overall results.

\subsection{Evaluation of the effective theory for arbitrary $N_{c}$}

We employ our results from chapter 4 which contain an evaluation of the effective theory in the strong coupling limit to order $\kappa^{4}$ in spatial hoppings. As we want to clearly separate the effects of different orders, we do not use the logarithmic resummation in the evaluation of the free energy. We also neglect terms containing $\bar{h}_{1}$, since they are suppressed exponentially at low temperatures. Despite these approximations one can already observe important aspects of baryon dynamics and we will discuss the modifications due to the neglected couplings later. Expanding equation (4.186) to $\mathcal{O}\left(\kappa^{4}\right)$ and setting all $\kappa^{(i)}=\kappa$ and $\mu^{(i)}=\mu$ results in the free energy density (this fixes errors occurring in equation (3.6) in [93]):

$$
\begin{aligned}
-f= & \log \left(z_{0}\right)+3 \Phi\left(\mathbf{n}_{1} \bullet \bullet \mathbf{n}_{2}\right)+3 \Phi\left(\mathbf{n}_{1} \bullet \mathbf{n}_{2} \bullet \mathbf{n}_{3}\right)+12 \Phi\left(\mathbf{n}_{1} \bullet \bullet_{2}\right) \\
& \left.-\frac{33}{2} \Phi\left(\mathbf{n}_{1} \bullet \bullet \mathbf{n}_{2}\right)^{2}\right) \\
= & \log \left(z_{0}\right)-3 \frac{\kappa^{2} N_{\tau}}{N_{c}} \frac{2 N_{f} z_{(11)}^{2}}{z_{0}^{2}} \\
& +3 \frac{\kappa^{4} N_{\tau}}{2\left(N_{c}^{2}-1\right)} \frac{4 N_{f}^{2} z_{(22)}^{2}-4 N_{f} z_{(11)^{2}} z_{(22)}+4 N_{f}^{2} z_{(11)^{2}}^{2}}{z_{0}^{2}} \\
& -3 \frac{\kappa^{4} N_{\tau}}{2 N_{c}\left(N_{c}^{2}-1\right)} \frac{8 N_{f}^{2} z_{(11)^{2}} z_{(22)}-2 N_{f} z_{(11)}^{2}-2 N_{f} z_{(22)}^{2}}{z_{0}^{2}} \\
& +3 \frac{\kappa^{4} N_{\tau}\left(N_{\tau}-1\right)}{2 N_{c}^{2}} \frac{4 N_{f}^{2} z_{(11)^{2}}^{2}+4 N_{f}^{2} z_{(21)}^{2}+4 N_{f} z_{(11)^{2}} z_{(21)}}{z_{0}^{2}} \\
& +15 \frac{\kappa^{4} N_{\tau}^{2}}{N_{c}^{2}} \frac{4 N_{f}^{2} z_{(11)}^{2} z_{(11)^{2}}}{z_{0}^{3}}+15 \frac{\kappa^{4} N_{\tau}\left(N_{\tau}-1\right)}{N_{c}^{2}} \frac{2 N_{f} z_{(11)}^{2} z_{(21)}}{z_{0}^{3}} \\
& +15 \frac{\kappa^{4} N_{\tau}}{N_{c}^{2}} \frac{2 N_{f} z_{(11)}^{2}\left(-z_{(22)}+2 z_{(11)}\right)}{z_{0}^{3}}-15 \frac{\kappa^{4} N_{\tau}}{N_{c}} \frac{4 N_{f} z_{(11)}^{2}}{z_{0}^{3}} \\
& +3 \frac{\kappa^{4} N_{\tau}}{N_{c}^{2}} \frac{4 N_{f} z_{(11)}^{2} z_{(11)}}{z_{0}^{3}}-3 \frac{\kappa^{4} N_{\tau}}{N_{c}} \frac{4 N_{f} z_{(11)}^{2}}{z_{0}^{3}}-\frac{33}{2} \frac{\kappa^{4} N_{\tau}^{2}}{N_{c}^{2}} \frac{4 N_{f} z_{(11)}^{4}}{z_{0}^{4}}
\end{aligned}
$$


where we have introduced the notation

$$
\begin{gathered}
z_{0}=\int_{\mathrm{SU}\left(N_{c}\right)} \mathrm{d} W \operatorname{det}\left(1+h_{1} W\right)^{2 N_{f}} \\
z_{\left(a_{1} b_{1}\right) \ldots\left(a_{k} b_{k}\right)}=\int_{\mathrm{SU}\left(N_{c}\right)} \mathrm{d} W \operatorname{det}\left(1+h_{1} W\right)^{2 N_{f}} \prod_{i=1}^{k} \operatorname{tr}\left(\frac{\left(h_{1} W\right)^{b_{i}}}{\left(1+h_{1} W\right)^{a_{i}}}\right) .
\end{gathered}
$$

We now need to evaluate the occurring integrals for general $N_{c}$. Note that when applying equation (C.14) to the derivation of the effective theory (see equation (4.57) for the contribution to $\left.\mathcal{O}\left(\kappa^{4}\right)\right)$ the determinants which arise are at least of order $\kappa^{N_{c}}$ and therefore are pushed to ever higher order when the large $N_{c}$ limit is considered. When these contributions are neglected, the integral reduces to the $U(N)$ case, meaning that for the derivation of the effective theory order by order in spatial hoppings, one can use the result of the one link integral for $U(N)$. When evaluating the effective theory, one has to integrate temporal gauge integrals. The temporal quark hoppings in positive direction are boosted by a factor of $e^{a \mu}$ and therefore the same simplifications do not apply when evaluating these integrals.

Nevertheless, the task is simplified by the fact that the integrands are all class functions of $\mathrm{SU}\left(N_{c}\right)$, meaning they are functions

$$
f: \mathrm{SU}\left(N_{c}\right) \rightarrow \mathbb{C}
$$

with the property that they are invariant under a change of basis in color space

$$
f(U)=f\left(V U V^{-1}\right) .
$$

We will first discuss some general rules for integrating class functions before focusing on the specific integrals we have to solve here.

\subsubsection{Integration of class functions}

As this chapter only deals with mathematical properties of the integration over class functions we will drop the index $c$ from $N_{c}$. $\mathrm{SU}(N)$ arises from $\mathrm{U}(N)$ via the restriction that the determinants of its elements evaluate to one. It is useful to first study the case without this restrictions, i.e. class functions on $\mathrm{U}(N)$. The strategy we use here is similar to [192], we just derive a slightly more general result needed for the integrals which occur in our case. Similar results have also been obtained using the method of orthonormal polynomials [193-195]. We also mention that it may be interesting to explore an alternative approach based on [196-198], especially considering section 6.3.2. See [199], for an application of this approach to integrals which are similar to the ones we are considering.

A class function $f$ on $\mathrm{U}(N)$ has the property that one can find a function $\tilde{f}$

$$
\tilde{f}:\left(S_{1}\right)^{N} \subset \mathbb{C}^{n} \rightarrow \mathbb{C}
$$

which, given the eigenvalues $\epsilon_{1}, \ldots, \epsilon_{N}$ of the $\mathrm{U}(N)$-matrix $U$, fulfills

$$
\tilde{f}\left(\epsilon_{1}, \ldots, \epsilon_{N}\right)=f(U)
$$

and is symmetric under the permutation of its arguments:

$$
\tilde{f}\left(\epsilon_{1}, \ldots, \epsilon_{N}\right)=\tilde{f}\left(\epsilon_{\sigma(1)}, \ldots, \epsilon_{\sigma(N)}\right) \quad \forall \sigma \in S_{N} .
$$

For such functions, the integral over $\mathrm{U}(N)$ can be written as an integral over angles parametrizing 
the eigenvalues [200]

$$
\int_{\mathrm{U}(N)} \mathrm{d} U f(U)=\frac{1}{N !(2 \pi)^{N}} \int_{0}^{2 \pi} \mathrm{d} \phi_{1} \cdots \int_{0}^{2 \pi} \mathrm{d} \phi_{N} \prod_{1 \leq k<l \leq N}\left|e^{i \phi_{l}}-e^{i \phi_{k}}\right|^{2} \tilde{f}\left(e^{i \phi_{1}}, \ldots, e^{i \phi_{N}}\right) .
$$

Introducing the Vandermonde determinant

$$
\begin{aligned}
\Delta\left(\epsilon_{1}, \ldots, \epsilon_{N}\right) & :=\operatorname{det}\left(V\left(\epsilon_{1}, \ldots, \epsilon_{N}\right)\right) \\
& :=\operatorname{det}_{1 \leq i, j \leq N}\left(\epsilon_{i}^{j-1}\right) \\
& =\prod_{1 \leq k<l \leq N}\left(\epsilon_{l}-\epsilon_{k}\right)
\end{aligned}
$$

the measure for the integration of the angles can be expressed in the following way:

$$
\prod_{1 \leq k<l \leq N}\left|e^{i \phi_{l}}-e^{i \phi_{k}}\right|^{2}=\Delta\left(\epsilon_{1}, \ldots, \epsilon_{N}\right) \Delta\left(\epsilon_{1}, \ldots, \epsilon_{N}\right)^{*}
$$

Now, since $\epsilon_{i}^{*}=1 / \epsilon_{i}$, one has

$$
\left(V^{\dagger} V\right)_{i j}=\sum_{k=1}^{N} \epsilon_{k}^{j-i}
$$

Therefore, by exploiting the multilinearity of determinants and the fact that determinants vanish when two rows or columns are linear dependent, one obtains

$$
\begin{aligned}
\prod_{1 \leq l<q \leq N}\left|e^{i \phi_{q}}-e^{i \phi_{l}}\right|^{2} & =\operatorname{det}_{1 \leq i, j \leq N}\left(\sum_{k=1}^{N} \epsilon_{k}^{j-i}\right) \\
& =\sum_{k_{1}, \ldots, k_{N}=1}^{N} \operatorname{det}_{1 \leq i, j \leq N}\left(\epsilon_{k_{i}}^{j-i}\right) \\
& =\sum_{\sigma \in S_{n}} \operatorname{det}_{1 \leq i, j \leq N}\left(\epsilon_{\sigma(i)}^{j-i}\right)
\end{aligned}
$$

Appropriate renaming of the integration variables and the symmetry of $f$ then give

$$
\begin{aligned}
\int_{\mathrm{U}(N)} \mathrm{d} U f(U) & =\frac{1}{N !(2 \pi)^{N}} \int_{0}^{2 \pi} \mathrm{d} \phi_{1} \cdots \int_{0}^{2 \pi} \mathrm{d} \phi_{N} \sum_{\sigma \in S_{n}} \operatorname{det}_{1 \leq i, j \leq N}\left(\epsilon_{\sigma(i)}^{j-i}\right) \tilde{f}\left(\epsilon_{1}, \ldots, \epsilon_{N}\right) \\
& =\frac{1}{N !(2 \pi)^{N}} \int_{0}^{2 \pi} \mathrm{d} \phi_{1} \cdots \int_{0}^{2 \pi} \mathrm{d} \phi_{N} \sum_{\sigma \in S_{n}} \operatorname{det}_{1 \leq i, j \leq N}\left(\epsilon_{i}^{j-i}\right) \tilde{f}\left(\epsilon_{\sigma(1)}, \ldots, \epsilon_{\sigma(N)}\right) \\
& =\frac{1}{(2 \pi)^{N}} \int_{0}^{2 \pi} \mathrm{d} \phi_{1} \cdots \int_{0}^{2 \pi} \mathrm{d} \phi_{N} \operatorname{det}_{1 \leq i, j \leq N}\left(\epsilon_{i}^{j-i}\right) \tilde{f}\left(\epsilon_{1}, \ldots, \epsilon_{N}\right) .
\end{aligned}
$$

For the evaluation of the effective theory it is furthermore enough to consider only those $f$ whose $\tilde{f}$ factorizes in the following way

$$
\tilde{f}\left(\epsilon_{1}, \ldots, \epsilon_{N}\right)=\sum_{\mu=1}^{N_{c}} \tilde{f}_{1, \mu}\left(\epsilon_{1}\right) \cdots \tilde{f}_{N, \mu}\left(\epsilon_{N}\right) .
$$


In that case, the multilinearity of the determinant can be invoked once more to obtain

$$
\int_{\mathrm{U}(N)} \mathrm{d} U f(U)=\frac{1}{(2 \pi)^{N}} \sum_{\mu=1}^{N_{c}} \int_{0}^{2 \pi} \mathrm{d} \phi_{1} \cdots \int_{0}^{2 \pi} \mathrm{d} \phi_{N} \operatorname{det}_{1 \leq i, j \leq N}\left(\tilde{f}_{i, \mu}\left(\epsilon_{i}\right) \epsilon_{i}^{j-i}\right) .
$$

Note that every row of the determinant in the last equation depends on a different $\phi_{i}$, so one can pull the integration into the determinant (this can easily be seen by Laplace expansion for example):

$$
\int_{\mathrm{U}(N)} \mathrm{d} U f(U)=\frac{1}{(2 \pi)^{N}} \sum_{\mu=1}^{N_{c}} \operatorname{det}_{1 \leq i, j \leq N}\left(\int_{0}^{2 \pi} \mathrm{d} \phi_{i} \tilde{f}_{i, \mu}\left(\epsilon_{i}\right) \epsilon_{i}^{j-i}\right) .
$$

Finally, we are interested in integrals over $\mathrm{SU}(N)$. It is possible to rewrite an $\mathrm{SU}(N)$ integral as a modified $\mathrm{U}(N)$ integral by making use of the group isomorphism $\mathrm{U}(N) \cong \mathrm{U}(1) \times \mathrm{SU}(N) / Z(N)$ [201]. To obtain the integral for the $\mathrm{SU}(N)$ case, one simply has to insert a $2 \pi$-periodic delta function into the the angular integrations

$$
\delta_{p}\left(\sum_{i=1}^{N} \phi_{i}\right)=\sum_{k=-\infty}^{\infty} \delta\left(\sum_{i=1}^{N} \phi_{i}-2 \pi k\right)=\frac{1}{2 \pi} \sum_{q=-\infty}^{\infty} e^{i q \sum_{i=1}^{N} \phi_{i}}
$$

which takes care of the constraint $\operatorname{det}(U)=1$ and can be expanded in a Fourier series. Including this constraint in equation (6.24) then leads to

$$
\int_{\mathrm{SU}(N)} \mathrm{d} U f(U)=\frac{1}{(2 \pi)^{N}} \sum_{\mu=1}^{N} \sum_{q=-\infty}^{\infty} \operatorname{det}_{1 \leq i, j \leq N}\left(\int_{0}^{2 \pi} \mathrm{d} \phi_{i} \tilde{f}_{i, \mu}\left(\epsilon_{i}\right) \epsilon_{i}^{j-i+q}\right) .
$$

\subsubsection{Evaluation of the temporal gauge integrals to $\mathcal{O}\left(\kappa^{4}\right)$}

Equation (6.27) gives us the tool to solve the $\mathrm{SU}\left(N_{c}\right)$ integrals that come in equation (6.3). Before we employ it, it is useful to note that for a non-singular matrix $A(t)$ depending on a real parameter one has

$$
\begin{aligned}
\frac{\mathrm{d}}{\mathrm{d} t} \operatorname{det}(A(t)) & =\operatorname{det}(A(t)) \operatorname{tr}\left(A(t)^{-1} \frac{\mathrm{d} A(t)}{\mathrm{d} t}\right), \\
\frac{\mathrm{d}}{\mathrm{d} t} A(t)^{-1} & =-A(t)^{-1} \frac{\mathrm{d} A(t)}{\mathrm{d} t} A(t)^{-1}, \\
\frac{\mathrm{d}}{\mathrm{d} t} \operatorname{tr}(A(t)) & =\operatorname{tr}\left(\frac{\mathrm{d} A(t)}{\mathrm{d} t}\right)
\end{aligned}
$$

and therefore the integrals in equation (6.3) are related in the following way

$$
\begin{aligned}
z_{(11)} & =\frac{h_{1}}{2 N_{f}} \frac{\partial}{\partial h_{1}} z_{0} \\
z_{(21)} & =z_{(11)}-z_{(22)} \\
z_{(11)^{2}} & =\frac{h_{1}}{2 N_{f}} \frac{\partial}{\partial h_{1}} z_{(11)}-\frac{1}{2 N_{f}} z_{(21)} .
\end{aligned}
$$

Consequently it is enough to only solve the integrals $z_{0}$ and $z_{(21)}$, which we will do now. 


\section{Determination of $z_{0}$}

Written in terms of its eigenvalues the integrand in $z_{0}$ reads

$$
\begin{aligned}
\operatorname{det}\left(1+h_{1} W\right)^{2 N_{f}} & =\prod_{i=1}^{N_{c}}\left(1+h_{1} \epsilon_{i}\right)^{2 N_{f}} \\
& =\prod_{i=1}^{N_{c}} \sum_{k}\left(\begin{array}{c}
2 N_{f} \\
k
\end{array}\right) h_{1}^{k} \epsilon_{i}^{k} .
\end{aligned}
$$

We intentionally put no restrictions on the sum over $k[96]$ as they are automatically implemented by the usual generalization of the binomial coefficient, where $\left(\begin{array}{c}2 N_{f} \\ k\end{array}\right)=0$ if $k>2 N_{f}$ or $k<0$. Applying equation (6.27) then gives

$$
z_{0}=\frac{1}{(2 \pi)^{N_{c}}} \sum_{q=-\infty}^{\infty} \operatorname{det}_{1 \leq i, j \leq N}\left(\int_{0}^{2 \pi} \mathrm{d} \phi_{i} \sum_{k}\left(\begin{array}{c}
2 N_{f} \\
k
\end{array}\right) h_{1}^{k} \epsilon_{i}^{j-i+q+k}\right) .
$$

For the integration over $\phi_{i}$ not to vanish, one needs

$$
0=j-i+q+k
$$

which allows us to get rid of the summation inside of the determinant

$$
z_{0}=\sum_{q=-\infty}^{\infty} \operatorname{det}_{1 \leq i, j \leq N_{c}}\left(\left(\begin{array}{c}
2 N_{f} \\
i-j-q
\end{array}\right) h_{1}^{i-j-q}\right) .
$$

Let's focus on the determinant alone, multilinearity and the Leibniz formula enable us to remove $h_{1}$-factors inside the determinant

$$
\begin{aligned}
\operatorname{det}_{1 \leq i, j \leq N_{c}}\left(\left(\begin{array}{c}
2 N_{f} \\
i-j-q
\end{array}\right) h_{1}^{i-j-q}\right) & =\operatorname{det}_{1 \leq i, j \leq N_{c}}\left(\left(\begin{array}{c}
2 N_{f} \\
i-j-q
\end{array}\right) h_{1}^{i-j}\right) h_{1}^{-N q} \\
& =\sum_{\sigma \in S_{N_{c}}} \prod_{i=1}^{N_{c}}\left(\begin{array}{c}
2 N_{f} \\
i-\sigma(i)-q
\end{array}\right) h_{1}^{\sum_{i=1}^{N_{c}} i-\sum_{i=1}^{N_{c}} \sigma(i)} h_{1}^{-N_{c} q} \\
& =\operatorname{det}_{1 \leq i, j \leq N_{c}}\left(\left(\begin{array}{c}
2 N_{f} \\
i-j-q
\end{array}\right)\right) h_{1}^{-N_{c} q} .
\end{aligned}
$$

This determinant vanishes for $q>0$ or $q<-2 N_{f}$, which can be seen by inspecting the determinant, but it is also clear from the discussion in section 4.6.3. Therefore we make the variable change $p=-q$ in the summation and observe that under the identifications $A=2 N_{f}$ and $L_{i}=i+p$ the determinant can be evaluated using equation (D.17)

$$
\operatorname{det}_{1 \leq i, j \leq N}\left(\left(\begin{array}{c}
2 N_{f} \\
i-j+p
\end{array}\right)\right)=\prod_{i=1}^{N_{c}} \frac{\left(2 N_{f}+N_{c}-i\right) \underline{p}}{(i+p-1) !} \prod_{1 \leq i<j \leq N}(j-i) .
$$

The last product can be rewritten to

$$
\prod_{1 \leq i<j \leq N_{c}}(j-i)=\prod_{j=2}^{N_{c}} \prod_{i=1}^{j-1}(j-i)
$$


6 Large $N_{c}$ behavior of the effective theory, quarkyonic matter

$$
\begin{aligned}
& =\prod_{j=2}^{N_{c}}(j-1) ! \\
& =\prod_{i=1}^{N_{c}}(i-1) !
\end{aligned}
$$

resulting in

$$
\begin{aligned}
\operatorname{det}_{1 \leq i, j \leq N_{c}}\left(\left(\begin{array}{c}
2 N_{f} \\
i-j+p
\end{array}\right)\right) & =\prod_{i=1}^{N_{c}} \frac{\left(2 N_{f}+N_{c}-i\right) \underline{p}}{(p+i-1) !}(i-1) ! \\
& =\prod_{i=1}^{N_{c}} \frac{\left(2 N_{f}+N_{c}-i\right)^{\underline{p}}}{(p+i-1)^{\underline{p}}} \\
& =\prod_{i=1}^{N_{c}} \frac{\left(2 N_{f}+i-1\right)^{\underline{p}}}{(p+i-1)^{\underline{p}}}
\end{aligned}
$$

When $N_{c}$ is large, it is useful to rewrite this result in a way such that no products or sums which have their limit at $N_{c}$ occur. This can be achieved by using the product analog of telescoping sums

$$
\prod_{i=1}^{N} \frac{a_{i}}{a_{i-1}}=\frac{a_{N}}{a_{0}}
$$

which leads to

$$
\begin{aligned}
\prod_{i=1}^{N_{c}} \frac{\left(2 N_{f}+i-1\right)^{\underline{p}}}{(p+i-1)^{\underline{p}}} & =\prod_{i=1}^{N_{c}} \prod_{j=1}^{p} \frac{2 N_{f}+i-j}{p+i-j} \\
& =\prod_{i=1}^{N_{c}} \prod_{j=1}^{p} \frac{\left(2 N_{f}-p\right)+p+i-j}{p+i-j} \\
& =\prod_{i=1}^{N_{c}} \prod_{j=1}^{p} \prod_{k=1}^{2 N_{f}-p} \frac{p+i-j+k}{p+i-j+k-1} \\
& =\prod_{i=1}^{N_{c}} \prod_{j=1}^{p} \prod_{k=1}^{2 N_{f}-p} \frac{i+j+k-1}{i+j+k-2} \\
& =\prod_{j=1}^{p} \prod_{k=1}^{2 N_{f}-p} \frac{N_{c}+j+k-1}{j+k-1} \\
& =\prod_{j=1}^{p} \frac{\left(N_{c}+j\right)^{\overline{2 N_{f}-p}}}{j^{2 N_{f}-p}} .
\end{aligned}
$$

Therefore, our final result reads

$$
z_{0}=\sum_{p=0}^{2 N_{f}}\left(\prod_{j=1}^{p} \frac{\left(N_{c}+j\right)^{\overline{2 N_{f}-p}}}{j^{\overline{2 N_{f}-p}}}\right) h_{1}^{p N_{c}}
$$

To illustrate the result consider the free energy density in the static limit for the case $N_{f}=1$ :

$$
-f_{\mathrm{LO}}=\log \left(1+\left(N_{c}+1\right) h_{1}^{N_{c}}+h_{1}^{2 N_{c}}\right) .
$$


Note that just like in the static case for $N_{f}=2$ and $N_{c}=3$ discussed in section section 5.3, the prefactors multiplying the powers of $h_{1}^{N_{c}}$ can be understood from spin degeneracy. For example, a color neural state consisting of $N_{c}$ fermions can only be antisymmetric in color space under particle exchange. To get an antisymmetric state, it therefore has to be completely symmetric in spin space, which means for $N_{c}$ spin $1 / 2$ particles that the spin quantum number of the composite state is $s=N_{c} / 2$. There are $N_{c}+1$ degenerate states with this spin quantum number, namely those with $N_{c} / 2 \leq s_{z} \leq N_{c} / 2$, which explains the $N_{c}+1$ prefactor multiplying $h_{1}^{N_{c}}$.

\section{Determination of $z_{(21)}$}

For the determination of $z_{(21)}$ we have to compute

$$
z_{(21)}=\int_{\mathrm{SU}(N)} \mathrm{d} W \operatorname{det}\left(1+h_{1} W\right)^{2 N_{f}} \operatorname{tr}\left(\frac{h_{1} W}{\left(1+h_{1} W\right)^{2}}\right) .
$$

The straightforward way to treat this integral is to write the integrand in its diagonal form in the following way

$$
\begin{aligned}
\operatorname{det}\left(1+h_{1} W\right)^{2 N_{f}} \operatorname{tr}\left(\frac{h_{1} W}{\left(1+h_{1} W\right)^{2}}\right) & =\prod_{i=1}^{N_{c}}\left(1+h_{1} \epsilon_{i}\right)^{2 N_{f}} \sum_{\mu=1}^{N_{c}} \frac{h_{1} \epsilon_{\mu}}{\left(1+h_{1} \epsilon_{\mu}\right)^{2}} \\
& =\sum_{\mu=1}^{N_{c}}\left(\prod_{i \neq \mu}\left(1+h_{1} \epsilon_{i}\right)^{2 N_{f}}\right)\left(1+h_{1} \epsilon_{\mu}\right)^{2 N_{f}-2} h_{1} \epsilon_{\mu} .
\end{aligned}
$$

However, when we apply equation (6.27), the result then is

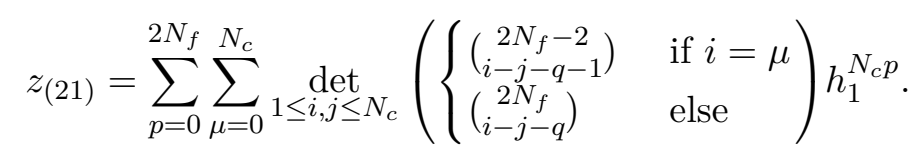

We do not know how to evaluate this determinant directly, notably equation (D.17) cannot be applied.

We circumvent this problem by expanding $\operatorname{tr}\left(h_{1} W\left(1+h_{1} W\right)^{-2}\right)$ around $h_{1}=0$. In general for $n, m \in \mathbb{N}^{*}$ one has in terms of eigenvalues

$$
\begin{aligned}
\operatorname{det}\left(1+h_{1} W\right)^{2 N_{f}} \operatorname{tr}\left(\frac{\left(h_{1} W\right)^{m}}{\left(1+h_{1} W\right)^{n}}\right) & =\left(\prod_{i=1}^{N_{c}}\left(1+h_{1} \epsilon_{i}\right)^{2 N_{f}}\right) \sum_{\mu=1}^{N_{c}} \frac{\left(h_{1} \epsilon_{\mu}\right)^{m}}{\left(1+h_{1} \epsilon_{\mu}\right)^{n}} \\
& =\sum_{\mu=1}^{N_{c}} \sum_{r=0}^{\infty}(-1)^{r}\left(\begin{array}{c}
n+r-1 \\
r
\end{array}\right)\left(h_{1} z_{\mu}\right)^{m+r}\left(\prod_{i=1}^{N_{c}}\left(1+h_{1} \epsilon_{i}\right)^{2 N_{f}}\right) .
\end{aligned}
$$

Consequently, equation (6.27) gives

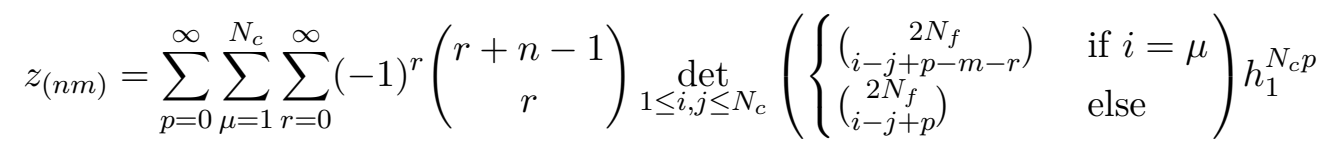

and the determinant occurring in the last equation can be evaluated using equation (D.17) under the identification $A=2 N_{f}$ and $L_{i}=\delta(i=\mu)(i+p-m-r)+\delta(i \neq \mu)(i+p)$. Tedious algebra then shows that in the case $n=2, m=1$ the summations over $r$ and $\mu$ decouple (in general this is 
not the case):

$$
\begin{aligned}
z_{(21)}= & \sum_{p=0}^{2 N_{f}} h_{1}^{N_{c} p} \operatorname{det}_{1 \leq i, j \leq N_{c}}\left(\left(\begin{array}{c}
2 N_{f} \\
i-j+p
\end{array}\right)\right) \\
& \times \sum_{\mu=1}^{N_{c}} \frac{(\mu+p-1)^{\underline{\mu}}}{\left(N_{c}-\mu\right) !(\mu-1) !\left(2 N_{f}-p+N_{c}\right)^{\underline{\mu}}} \\
& \times \sum_{r=0}^{p-1} \frac{\left(r+N_{c}\right) \frac{N_{c}}{\left(2 N_{f}-p+1\right)^{\underline{r}}}}{\left(N_{c}+r\right)^{\underline{r}}} .
\end{aligned}
$$

There are algorithms to find closed form expressions for sums like the ones over $\mu$ and $r$ in the last equation [202]. Luckily they have been implemented in computer algebra systems, asking Mathematica for advice, we learn that

$$
\begin{aligned}
\sum_{\mu=1}^{N_{c}} \frac{(\mu+p-1)^{\underline{\mu}}}{\left(N_{c}-\mu\right) !(\mu-1) !\left(2 N_{f}-p+N_{c}\right)^{\underline{\mu}}} & =\frac{p\left(N_{c}+2 N_{f}\right) !\left(2 N_{f}-p\right) !}{\left(N_{c}-1\right) !\left(2 N_{f}+1\right) !\left(N_{c}+2 N_{f}-p\right) !}, \\
\sum_{r=0}^{p-1} \frac{\left(r+N_{c}\right) \frac{N_{c}}{\left(2 N_{f}-p+1\right)^{\underline{r}}}}{\left(N_{c}+r\right)^{\underline{r}}} & =\frac{N_{c} !\left(2-2 N_{f}\right)^{\overline{p-1}}}{\left(-N_{c}-2 N_{f}+1\right)^{\overline{p-1}}} .
\end{aligned}
$$

The product of these sums then simplifies neatly leading to the end result

$$
z_{(21)}=\sum_{p=0}^{2 N_{f}} h_{1}^{N_{c} p} \operatorname{det}_{1 \leq i, j \leq N_{c}}\left(\left(\begin{array}{c}
2 N_{f} \\
i-j+p
\end{array}\right)\right) \frac{p N_{c}}{2 N_{f}} \frac{\left(2 N_{f}+N_{c}\right)\left(2 N_{f}-p\right)}{\left(2 N_{f}+1\right)\left(2 N_{f}-1\right)} .
$$

We now have computed all integrals necessary for the free energy density to $\mathcal{O}\left(\kappa^{4}\right)$ for arbitrary $N_{c} \geq 3$, and following section 5.2 the same is true for all thermodynamic functions. In contrast to section 5.2 , we have here $\mu_{B}=N_{c} \mu$ and the baryon masses are to leading order

$$
a m_{B}^{\mathrm{LO}}=N_{c} a m=-N_{c} \log (2 \kappa) .
$$

The derivative of $\kappa$ with respect to $a$ is computed at constant baryon mass, which leads to

$$
a \frac{\partial \kappa}{\partial a}=\kappa \ln (2 \kappa) .
$$

\subsection{The onset transition in the large $N_{c}$ limit}

We can now begin to investigate properties of the theory. Figure 6.2 shows the onset transition based on the calculations so far. One can clearly observe that the transition steepens when $N_{c}$ is increased, which extrapolates to a step function for asymptotically large values of $N_{c}$, corresponding to a first order transition. This asymptotic behavior is explicitly shown in the next section. For lower values of $N_{\tau}$, which means higher temperatures, the curve is flattened for each value of $N_{c}$ in comparison to larger values of $N_{\tau}$. However for asymptotically large $N_{c}$ this curve will always become a step function, making the transition always first order independent of temperature. Note that we use the leading order expression for the baryon mass to express our results as we do not know the baryon mass in the hopping expansion for general $N_{c}$. For this reason the onset happens exactly at $\mu_{B}=m_{B}^{\mathrm{LO}}$ in the strong coupling limit. We remark that for higher temperature one should also discuss the inclusion $\bar{h}_{1}$ and gauge corrections, which we have neglected so far. This is done in section 6.3.2. Furthermore an important point is that similar to the large $N_{\tau}$ limit in 

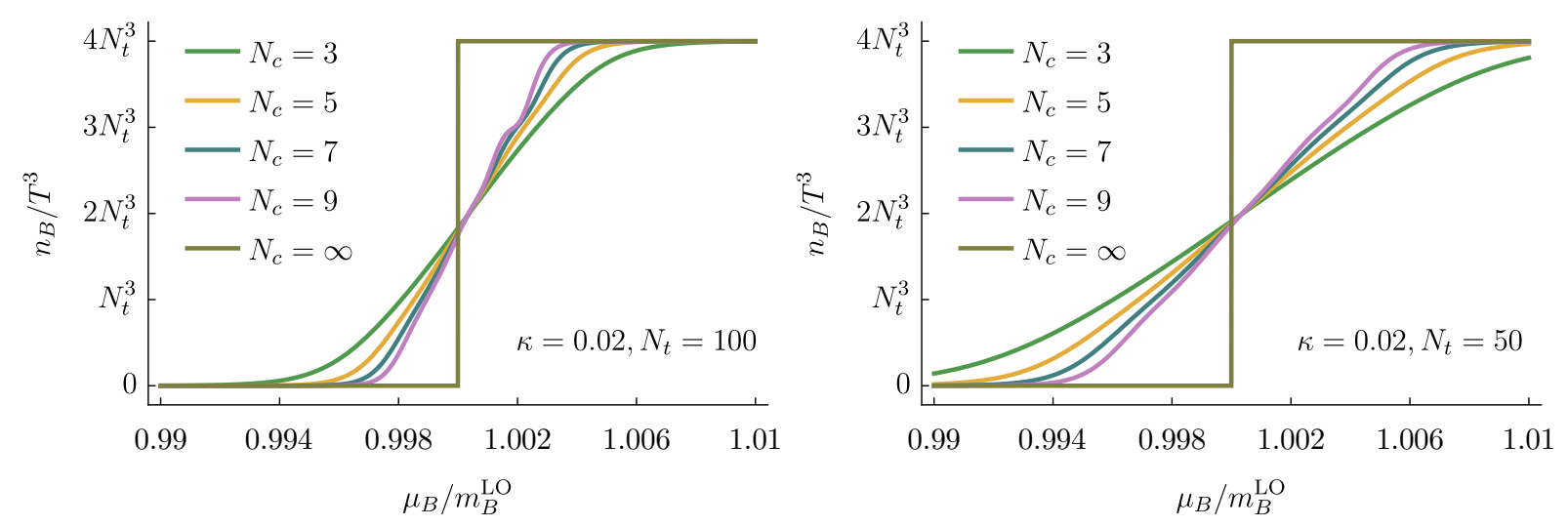

Figure 6.2: Adapted from [93]. Onset transition for different values of $N_{c}$ and $N_{\tau}$ in the strong coupling regime.

the static strong coupling limit for finite $N_{c}$, the transition we observe here for infinite $N_{c}$ is from vacuum to a saturated lattice, which is a discretization artifact. Approaching the continuum, the saturation density is moved to larger physical values and we will investigate this in more detail in section 6.3.4.

\subsection{Thermodynamic observables for large $N_{c}$}

In the previous sections we have obtained the free energy density for general $N_{c}$. Naturally, the same is true for all thermodynamic observables derived from it. From this, we can easily obtain their asymptotic behavior in the limit $N_{c} \rightarrow \infty$. We already noted that the lattice saturation heavily influences the results for large $N_{c}$. We know that the unphysical lattice saturation is contained in the static limit, while the corrections in $\kappa$ modify how the curves enter the high and low density asymptotes. The latter effects influence how the continuum is approached in the theory and are therefore physically significant. Therefore, to be able to distinguish these effects clearly, we study the different orders in the hopping expansion separately.

Before discussing our results for $N_{f}=2$, we describe the general strategy to obtain the asymptotic behavior. An example which illustrates the general procedure is the $\kappa^{2}$ correction to the pressure:

$$
a^{4} p_{1}=-6 \kappa^{2} \frac{\left(\frac{1}{2} N_{c}\left(N_{c}+1\right) h_{1}^{N_{c}}+N_{c} h_{1}^{2 N_{c}}\right)^{2}}{N_{c}\left(1+h_{1}^{N_{c}}\left(1+N_{c}\right)+h_{1}^{2 N_{c}}\right)^{2}} .
$$

When $h_{1}<1$ the term $h_{1}^{N_{c}}$ vanishes faster than any power of $N_{c}$ that can occur as a prefactor and to obtain the asymptotic behavior in this case we can therefore consider the Taylor expansion around $h_{1}^{N_{c}}=0$ :

$$
\begin{aligned}
a^{4} p_{1} & =-\frac{3}{2} \kappa^{2} N_{c}\left(N_{c}+1\right)^{2} h_{1}^{2 N_{c}}+\mathcal{O}\left(h_{1}^{3 N_{c}}\right) \\
& \sim-\frac{3}{2} \kappa^{2} N_{c}^{3} h_{1}^{2 N_{c}} \text { for } N_{c} \rightarrow \infty
\end{aligned}
$$

For $h_{1}>1$ we extend the fraction by $h_{1}^{4 N_{c}}$ to obtain

$$
a^{4} p_{1}=-6 \kappa^{2} \frac{\left(\frac{1}{2} N_{c}\left(N_{c}+1\right) \frac{1}{h_{1}^{N_{c}}}+N_{c}\right)^{2}}{N_{c}\left(\frac{1}{h_{1}^{2 N_{c}}}+\frac{1}{h_{1}^{N_{c}}}\left(1+N_{c}\right)+1\right)^{2}} .
$$




\begin{tabular}{|c|c|c|c|c|}
\hline \multicolumn{2}{|c|}{ Order hopping expansion } & $\kappa^{0}$ & $\kappa^{2}$ & $\kappa^{4}$ \\
\hline \multirow{4}{*}{$h_{1}<1$} & $a^{4} p$ & $\sim \frac{1}{6 N_{\tau}} N_{c}^{3} h_{1}^{N_{c}}$ & $\sim-\frac{1}{48} N_{c}^{7} h_{1}^{2 N_{c}}$ & $\sim \frac{N_{\tau}}{240} N_{c}^{8} h_{1}^{2 N_{c}}$ \\
\hline & $a^{3} n_{B}$ & $\sim \frac{1}{6} N_{c}^{3} h_{1}^{N_{c}}$ & $\sim-\frac{N_{\tau}}{24} N_{c}^{7} h_{1}^{2 N_{c}}$ & $\sim \frac{N_{\tau}^{2}}{120} N_{c}^{8} h_{1}^{2 N_{c}}$ \\
\hline & $a^{4} e$ & $\sim-\frac{\ln (2 \kappa)}{6} N_{c}^{4} h_{1}^{N_{c}}$ & $\sim \frac{N_{\tau} \ln (2 \kappa)}{48} N_{c}^{8} h_{1}^{2 N_{c}}$ & \\
\hline & $\epsilon$ & 0 & $\sim-\frac{1}{4} N_{c}^{3} h_{1}^{N_{c}}$ & \\
\hline \multirow{4}{*}{$h_{1}>1$} & $a^{4} p$ & $\sim \frac{4 \ln \left(h_{1}\right)}{N_{\tau}} N_{c}$ & $\sim-12 N_{c}$ & $\sim 78 N_{c}$ \\
\hline & $a^{3} n_{B}$ & $\sim 4$ & $\sim-N_{\tau} \frac{N_{c}^{4}}{h_{1}^{N_{c}}}$ & $\sim-3 N_{\tau}^{2} \frac{N_{c}^{5}}{h_{1}^{N_{c}}}$ \\
\hline & $a^{4} e$ & $\sim-4 \ln (2 \kappa) N_{c}$ & $\sim 24 \ln (2 \kappa) N_{c}$ & \\
\hline & $\epsilon$ & 0 & $\sim-6$ & \\
\hline
\end{tabular}

Table 6.1: Adapted from [93], differences are due to the errors fixed in equation (6.3). Large $N_{c}$ behavior of the thermodynamic functions and the interaction energy per baryon, order by order in the hopping expansion, on both sides of the onset transition for $N_{f}=2$.

Now, $1 / h_{1}^{N_{c}}$ is a good expansion parameter and expanding around $1 / h_{1}^{N_{c}}=0$ gives

$$
\begin{aligned}
a^{4} p_{1} & =-6 \kappa^{2} N_{c}+\mathcal{O}\left(1 / h_{1}^{N_{c}}\right) \\
& \sim-6 \kappa^{2} N_{c} \text { for } N_{c} \rightarrow \infty .
\end{aligned}
$$

We remark that in contrast to the $\kappa^{2}$ contribution, there are several $\kappa^{4}$ terms in equation (6.3). Applying the above strategy term by term one does obtain results that scale, for example, like $\sim N_{c}^{2}$ and it is only when the whole expression at $\kappa^{4}$ is considered, that the scaling $\sim N_{c}$ for $h_{1}>1$ is obtained. Furthermore, when neglecting terms that go like $N_{\tau} \kappa^{4}$, one also does not get the correct scaling.

We summarized the results of the procedure for $N_{f}=2$ in table 6.1. For $h_{1}<1$ all observables go like $\sim N_{c}^{k} h_{1}^{N_{c}}$, which means they are exponentially suppressed for increasing $N_{c}$ up to the onset. Therefore an amplification of the silver blaze property is observed when $N_{c}$ is increased.

More interestingly, for $h_{1}>1$, all contributions to the pressure scale like $p \sim N_{c}$, as we have seen above already. This scaling characterizes quarkyonic matter. While for the leading order the scaling trivially follows from lattice saturation, we already explained that the corrections do not contribute to saturation, and one can also see this explicitly in the table. The fact that the scaling holds for both corrections we calculated suggests that this may hold to all orders in $\kappa$, and therefore to be a genuine feature of strongly coupled QCD for all current quark masses. Note that at $\kappa^{2}$ there is a finite interaction energy per baryon relative to the baryon mass in the limit of infinite $N_{c}$, which is consistent with the predictions in [173]. From our investigations in section 5.4 we assume that this also holds for the $\kappa^{4}$-corrections in the case of baryon chemical potential.

\subsubsection{The transition region}

So far we only investigated what happens when $N_{c} \rightarrow \infty$ with $h_{1}$ fixed, i. e. with the quark chemical potential fixed. By adjusting the chemical potential such that $\mu-m \sim 1 / N_{c}$ we can consider also $h_{1}^{N_{c}} \sim 1$, which is just around the onset. In this case, the asymptotic behavior is determined by the prefactors in front of the powers of $h_{1}^{N_{c}}$ which are polynomials in $N_{c}$ as can be seen from 
equations (6.56) and (6.68). For the pressure one then obtains the following asymptotic behavior

$$
a^{4} p \sim \frac{4}{N_{\tau}} \log \left(N_{c}\right)-3 \kappa^{2} N_{c}+\frac{N_{\tau} \kappa^{4}}{10} N_{c}^{2}+\mathcal{O}\left(\kappa^{6}\right)
$$

This is an indication that the hopping expansion does not converge in this regime for large $N_{c}$. First signs of this behavior can be seen for $N_{c}=9$ in figure 6.2 , where uncontrolled wiggles form around the onset. Therefore, we do not have control over this parameter region in our expansion and cannot make predictions about it. On the other hand, the width of this region goes to zero for large $N_{c}$ and thus, does not affect our previous observations. Likewise, we cannot judge if this behavior is related to a percolation-type phase transition for some critical $N_{c}$, as predicted in [203, 204].

\subsubsection{Inclusion of $\bar{h}_{1}$-corrections}

As mentioned before, at higher temperatures, i. e. lower $N_{\tau}$, we also need to consider the inclusion of $\bar{h}_{1}$. Clearly, for $h_{1}>1$ the behavior is unchanged, as in that case the large $N_{c}$ behavior is determined by the largest powers of $h_{1}^{N_{c}}$. For $h_{1}<1$ on the other hand, one has to consider $\mu$-independent terms with equal powers of $h_{1}$ and $\bar{h}_{1}$. They are not necessarily exponentiated by powers of $N_{c}$, and therefore not suppressed when $N_{c} \rightarrow \infty$. Then again, these are mesonic contributions which are expected to scale as $\sim N_{c}^{0}$.

To show this explicitly, at least for the leading order contribution, we have to modify our calculation of $z_{0}$. The integral we have to solve in this case reads

$$
z_{0}=\int_{N_{c}} \mathrm{~d} W \operatorname{det}\left(1+h_{1} W\right)^{2 N_{f}} \operatorname{det}\left(1+\bar{h}_{1} W^{\dagger}\right)^{2 N_{f}}
$$

In terms of its eigenvalues, the integrand reads

$$
\begin{aligned}
\prod_{i=1}^{N_{c}}\left(1+h_{1} \epsilon_{i}\right)^{2 N_{f}}\left(1+\bar{h}_{1} \epsilon_{i}^{*}\right)^{2 N_{f}} & =\prod_{i=1}^{N_{c}}\left[\sum_{n}\left(\begin{array}{c}
2 N_{f} \\
n
\end{array}\right) h_{1}^{n} \epsilon_{i}^{n} \sum_{m}\left(\begin{array}{c}
2 N_{f} \\
m
\end{array}\right) \bar{h}_{1}^{m}\left(\epsilon_{i}^{*}\right)^{m}\right] \\
& =\prod_{i=1}^{N_{c}}\left[\sum_{n}\left(\begin{array}{c}
2 N_{f} \\
n
\end{array}\right) h_{1}^{n} \epsilon_{i}^{n} \sum_{m}\left(\begin{array}{c}
2 N_{f} \\
m
\end{array}\right) \bar{h}_{1}^{m}\left(\epsilon_{i}^{*}\right)^{m}\right] \\
& =\prod_{i=1}^{N_{c}}\left[\sum_{n, m}\left(\begin{array}{c}
2 N_{f} \\
n
\end{array}\right)\left(\begin{array}{c}
2 N_{f} \\
m
\end{array}\right) h_{1}^{n} \bar{h}_{1}^{m} \epsilon_{i}^{n-m}\right] .
\end{aligned}
$$

Again, we can now apply equation (6.27) to obtain

$$
\begin{aligned}
& z_{0}=\sum_{q=-\infty}^{\infty} \bar{h}_{1}^{N_{c} q} \operatorname{det}_{1 \leq i, j \leq N_{c}}\left(\sum_{n}\left(\begin{array}{c}
2 N_{f} \\
n
\end{array}\right)\left(\begin{array}{c}
2 N_{f} \\
n+q+i-j
\end{array}\right) h_{1}^{n} \bar{h}_{1}^{n}\right) \\
& =\sum_{q=-\infty}^{\infty} \bar{h}_{1}^{N_{c} q} \sum_{n_{1}, \ldots, n_{N_{c}}} \operatorname{det}_{1 \leq i, j \leq N_{c}}\left(\left(\begin{array}{c}
2 N_{f} \\
n_{i}
\end{array}\right)\left(\begin{array}{c}
2 N_{f} \\
n_{i}+q+i-j
\end{array}\right) h_{1}^{n_{i}} \bar{h}_{1}^{n_{i}}\right) \\
& =\sum_{q=-\infty}^{\infty} \bar{h}_{1}^{N_{c} q} \sum_{n_{1}, \ldots, n_{N_{c}}} \prod_{l=1}^{N_{c}}\left[\left(\begin{array}{c}
2 N_{f} \\
n_{i}
\end{array}\right) h_{1}^{n_{i}} \bar{h}_{1}^{n_{i}}\right] \operatorname{det}_{1 \leq i, j \leq N_{c}}\left(\left(\begin{array}{c}
2 N_{f} \\
n_{i}+q+i-j
\end{array}\right)\right),
\end{aligned}
$$

where we, once more, used the multilinearity of the determinant in the last two steps. The determinant can be evaluated using equation (D.17) by setting $L_{i}=n_{i}+p+i$ and $A=2 N_{f}$, resulting 
in

$$
z_{0}=\sum_{q=-\infty}^{\infty} \bar{h}_{1}^{N_{c} q} \sum_{n_{1}, \ldots, n_{N_{c}}} \prod_{l=1}^{N_{c}}\left[\left(\begin{array}{c}
2 N_{f} \\
n_{i}
\end{array}\right) h_{1}^{n_{i}} \bar{h}_{1}^{n_{i}}\right] \prod_{i=1}^{N_{c}} \frac{\left(2 N_{f}+N_{c}-i\right) \underline{n_{i}+p}}{\left(n_{i}+q+i-1\right) !} \prod_{1 \leq i, j \leq N_{c}}\left(n_{j}+j-n_{i}-i\right) .
$$

Unfortunately, we currently do not know how to simplify this result any further.

At least for $N_{f}=1$ the result is known [157] and leads to the pressure

$$
\begin{aligned}
a^{4} p_{h_{1}, \bar{h}_{1}}= & \log \left(\sum_{k=0}^{2 N_{c}} T(k)(2 \kappa)^{2 k N_{\tau}}\right. \\
& \left.+\sum_{k=0}^{N_{c}} P(k)(2 \kappa)^{\left(2 k+N_{c}\right) N_{\tau}} 2 \cosh \left(N_{c} \mu / T\right)+(2 \kappa)^{2 N_{c} N_{\tau}} 2 \cosh \left(2 N_{c} \mu / T\right)\right),
\end{aligned}
$$

with $T(k)=\left(\begin{array}{c}\min \left(k, 2 N_{c}-k\right)+3 \\ 3\end{array}\right)$ and $P(k)=\left(N_{c}+1-k\right)(k+1)$. We are interested here in $h_{1}<1$, which means $\mu<a \log (2 \kappa)$. From using the representation of cosh in terms of exp it is clear that in this case the $\mu$-dependent terms are exponentially suppressed in the large $N_{c}$ limit. The $\mu$-independent terms are more interesting. To compute their contribution, we note that

$$
\begin{aligned}
\sum_{k=0}^{2 N_{c}}\left(\begin{array}{c}
\min \left(k, 2 N_{c}-k\right)+3 \\
3
\end{array}\right)(2 \kappa)^{2 k N_{\tau}} & \sum_{k=0}^{N_{c}}\left(\begin{array}{c}
k+3 \\
3
\end{array}\right)(2 \kappa)^{2 k N_{\tau}}+\sum_{k=N_{c}+1}^{2 N_{c}}\left(\begin{array}{c}
2 N_{c}-k+3 \\
3
\end{array}\right)(2 \kappa)^{2 k N_{\tau}} \\
= & \sum_{k=0}^{N_{c}}\left(\begin{array}{c}
k+3 \\
3
\end{array}\right)(2 \kappa)^{2 k N_{\tau}}+\sum_{k=0}^{N_{c}-1}\left(\begin{array}{c}
k+3 \\
3
\end{array}\right)(2 \kappa)^{2\left(2 N_{c}-k\right) N_{\tau}} \\
= & \sum_{k=0}^{N_{c}}\left(\begin{array}{c}
k+3 \\
3
\end{array}\right)(2 \kappa)^{2 k N_{\tau}}+(2 \kappa)^{4 N_{c} N_{\tau}} \sum_{k=0}^{N_{c}-1}\left(\begin{array}{c}
k+3 \\
3
\end{array}\right)\left(\frac{1}{2 \kappa}\right)^{2 k N_{\tau}} .
\end{aligned}
$$

Both sums can be obtained by considering derivatives of the geometric sum (we remark that the geometric series cannot be applied to the latter sum) and in the limit we get

$$
\lim _{N_{c} \rightarrow \infty} \sum_{k=0}^{2 N_{c}}\left(\begin{array}{c}
\min \left(k, 2 N_{c}-k\right)+3 \\
3
\end{array}\right)(2 \kappa)^{2 k N_{\tau}}=\frac{1}{\left(1-(2 \kappa)^{2 N_{\tau}}\right)^{4}} .
$$

For the pressure this then implies

$$
\begin{aligned}
a^{4} p_{h_{1}, \bar{h}_{1}} & \sim-\frac{4}{N_{\tau}} \log \left(1-\left(2 \kappa^{2 N}\right)\right) \text { for } N_{c} \rightarrow \infty \\
& \sim N_{c}^{0}
\end{aligned}
$$

as claimed.

\subsubsection{Gauge corrections and 't Hooft scaling}

In the strong coupling limit we have $\beta=0$. To have a well defined 't Hooft coupling $\lambda_{H}=2 N_{c}^{2} / \beta$ and consequently a well defined 't Hooft limit, we have to consider what happens when $\beta>0$. Therefore we have to discuss the inclusion of gauge corrections. We will argue, that the qualitative properties of our observations will not change. 
As the inclusion of the leading corrections in [65] only makes use of equation (2.44) which is generic for $N_{c}$, we can use their results here. Replacing the character expansion coefficients by their correct generalization according to equation (2.43) the free energy density to $\mathcal{O}\left(\kappa^{2}\right)$ now reads

$$
-f=\log \left(z_{0}\left(h_{1}\right)\right)-3 \frac{\kappa^{2} N_{\tau}}{N_{c}}\left[1+2 \frac{u-u^{N_{t}}}{1-u}\right] \frac{2 N_{f} z_{(11)}^{2}}{z_{0}^{2}},
$$

where $h_{1}$ is now corrected by gauge contributions in the following way:

$$
h_{1}=(2 \kappa)^{N_{\tau}} e^{a \mu N_{\tau}} \exp \left[6 N_{\tau} \kappa^{2} \frac{u-u^{N_{\tau}}}{1-u}\right] .
$$

For $\lambda_{H}>1$, the 't Hooft then gives

$$
u(\beta)=\frac{1}{\lambda_{H}} .
$$

Therefore, our results concerning the asymptotic behavior of the thermodynamic functions is only modified by a constant $\sim N_{c}^{0}$.

For higher temperatures it is also relevant to consider leading order contributions from the pure gauge sector, which read [79]

$$
\prod_{\mathbf{n} \in \Lambda_{s}} \prod_{k=1}^{3}\left[1+\lambda_{1}\left(L(\mathbf{n}) L^{*}\left(\mathbf{n}+\mathbf{e}_{k}\right)+L^{*}(\mathbf{n}) L\left(\mathbf{n}+\mathbf{e}_{k}\right)\right)\right]
$$

with the effective coupling $\lambda_{1}$ that to first order in the character expansion has the expression

$$
\lambda_{1}=u^{N_{\tau}} .
$$

To leading order, this term changes $-f$ in equation (6.3) due to a mixing term with the static determinant, which reads

$$
-f_{\lambda_{1}, h_{1}}=6 \lambda_{1} \frac{z_{(01)} z_{(0-1)}}{z_{0}^{2}} .
$$

To obtain $z_{(01)}$ and $z_{(0-1)}$ one can again apply equation (6.27) and equation (D.17), no new challenges occur in comparison to the previous calculations. The result is

$$
\begin{aligned}
z_{(01)} & =\sum_{p=0}^{2 N_{f}} \operatorname{det}_{1 \leq i, j \leq N_{c}}\left(\left(\begin{array}{c}
2 N_{f} \\
i-j+p
\end{array}\right)\right) \frac{p N_{c}}{N_{c}+2 N_{f}-p} h_{1}^{p N_{c}} \\
z_{(0-1)} & =\sum_{p=0}^{2 N_{f}} \operatorname{det}_{1 \leq i, j \leq N_{c}}\left(\left(\begin{array}{c}
2 N_{f} \\
i-j+p
\end{array}\right)\right) \frac{\left(2 N_{f}-p\right) N_{c}}{N_{c}+p} h_{1}^{p N_{c}} .
\end{aligned}
$$

From equation (6.96) we conclude that $\lambda_{1}=\frac{1}{\lambda_{H}^{N_{\tau}}}$, which means that we can use the same strategy as in section 6.3 to obtain

$$
a^{4} p_{\lambda_{1}, h_{1}} \sim \frac{4}{N_{\tau} \lambda_{H}^{N_{\tau}}} N_{c}^{3}\left\{\begin{array}{ll}
h_{1}^{N_{c}}, & \text { if } h_{1}<1, \\
\frac{1}{h_{1}^{N_{c}}}, & \text { if } h_{1}>1,
\end{array} \text { for } N_{c} \rightarrow \infty .\right.
$$

For $h_{1}>1$ the asymptotic behavior of this term is subleading to those in table 6.1 and for $h_{1}<1$ the results in the table are only modified by a constant $\sim N_{c}^{0}$.

Corrections which are exclusively due to the gauge part of the action become relevant at $\mathcal{O}\left(\lambda_{1}^{4}\right)$. 

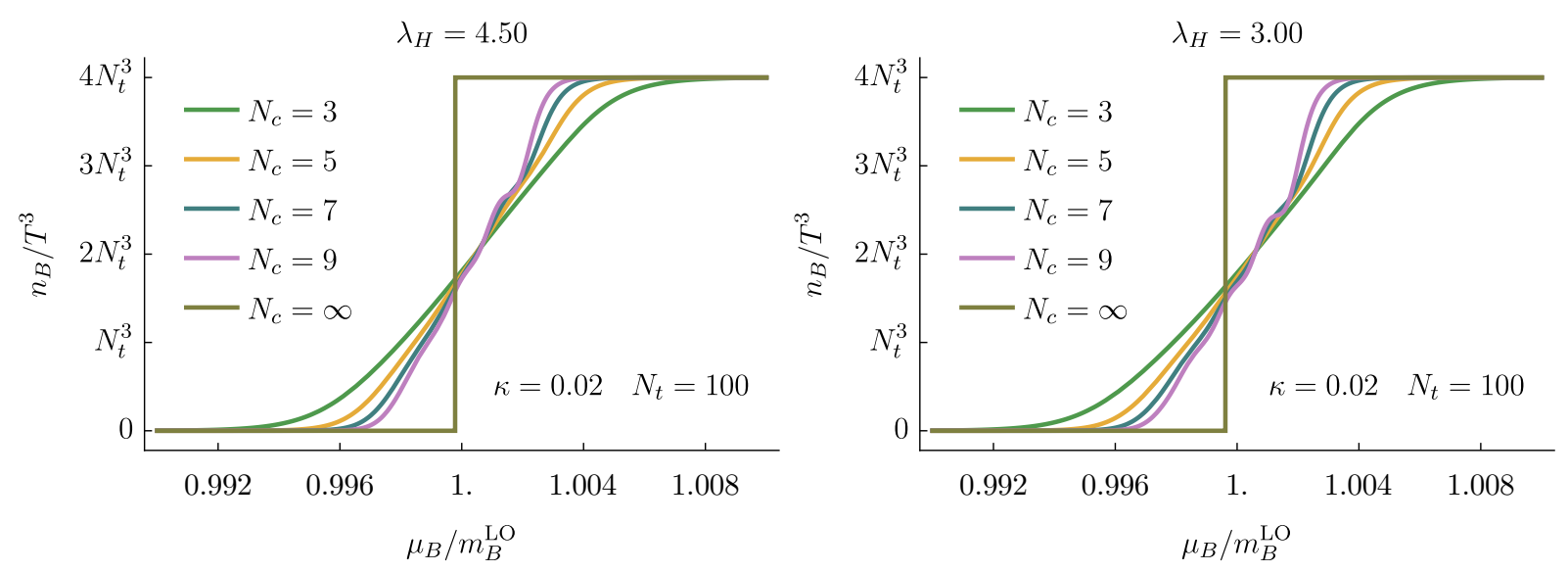

Figure 6.3: Adapted from [93]. Behavior of the Baryon density for growing $N_{c}$ after gauge corrections have been included. The gauge couplings are related by the 't Hooft coupling, which is kept fixed.

When taking the large $N_{c}$ limit order by order, only the following type of integrals

$$
\int_{\mathrm{SU}\left(N_{c}\right)} \mathrm{d} W \operatorname{tr}(W)^{n} \operatorname{tr}\left(W^{\dagger}\right)^{n}=n ! \text { for } n \leq N_{c}
$$

occur. Their contribution to the pressure is therefore $\mu$-independent and scales as $\sim \lambda_{1}^{k} \sim N_{c}^{0}$.

So far we only considered the leading order expression of $\lambda_{1}$, whose $N_{c^{c}}$-dependence is solely determined by $u$. The last statement would however be wrong if additional corrections to $\lambda_{1}$ introduce dependencies in $\lambda_{1}$ which do not correspond to character expansion coefficients. Corrections to $\lambda_{1}$ up to $\mathcal{O}\left(u^{8}\right)$ were obtained in [94]. One can observe that although some corrections indeed introduce additional $N_{c}$-dependencies, these additional factors cancel when the total contribution of these corrections is taken into account. Including characters of higher representations, similar observations have been made in [171], where the strong coupling expansion of pure gauge theory was explored without using an effective theory.

Figure 6.3 shows how figure 6.2 is modified when gauge corrections are included, for two different choices of the 't Hooft coupling. Clearly, only quantitatively small changes can be observed and the qualitative observations about the asymptotic behavior stay the same. Naturally, when including higher orders, more complicated contributions and interactions in the effective theory can arise, with non-trivial $N_{c}$ scaling. However, having $N_{c}$ in the exponent, the powers of $h_{1}^{N_{c}}$ should always be the dominant aspect for the large $N_{c}$ scaling behavior of baryons, as it was observed in the leading order contributions here.

\subsubsection{Towards the continuum}

If we look again, for example, at the argumentation around equation (6.103), we note that it was assumed so far that the large $N_{c}$ limit can be interchanged with the strong coupling expansion. Otherwise, one can imagine situations where contributions become relevant with $n>N_{c}$ in that integral. Indeed, this was observed in the case of QCD in $1+1$ dimensions $^{1}$ by Gross and Witten [205] and is also the reason why equation (6.96) is restricted to the case $\lambda_{H}>1$. They concluded that the interchange of the large $N_{c}$ limit and the strong coupling expansion should be deemed "highly suspicious". Furthermore, the fact that in our case the density immediately jumps to the value of the saturated lattice also suggests that the limits should be taken in the opposite order, 

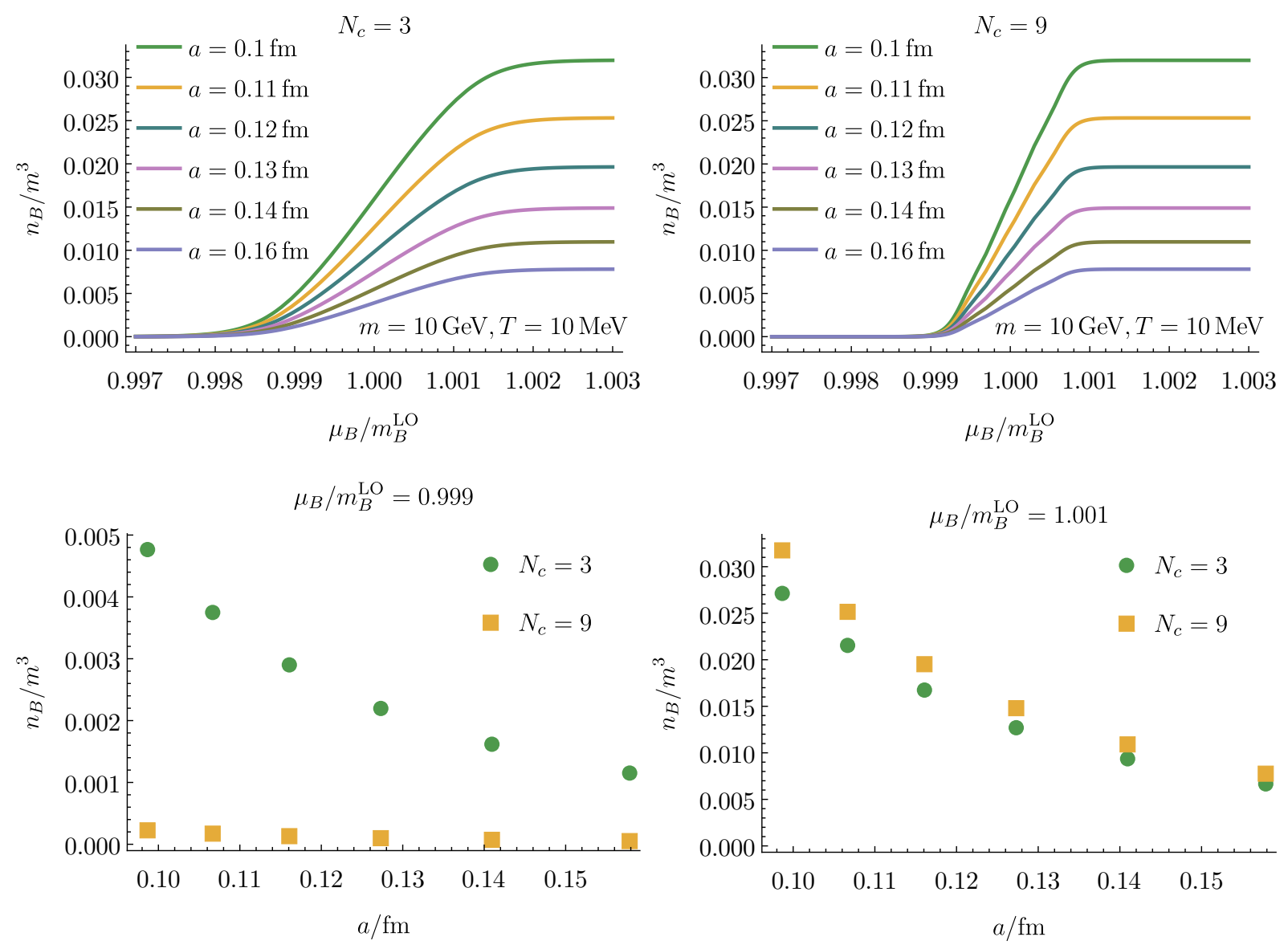

Figure 6.4: Adapted from [93]. Continuum approach for the baryon density. Steepening of the onset transition for growing $N_{c}$ is also observed if continuum limit is taken first.

to get results relevant for continuum physics.

While we are certainly not able to sum all contributions before taking the limit $N_{c} \rightarrow \infty$, we can still investigate how the baryon density behaves towards the continuum. First, we set a scale at $\mathrm{SU}(3)$, following the strategy in [65]. This means we use the non-perturbative beta-function of pure gauge theory to relate $\beta_{\mathrm{SU}(3)}$ to $a / r_{0}$, where $r_{0}$ is the Sommer parameter [206]. Although we do have quarks in our system, they are very heavy and therefore their influence on the running of the coupling should be relatively small. The value $r_{0}=0.5 \mathrm{fm}$ then relates the lattice parameters to a physical scale, whereby the temperature is determined via $T=1 /\left(a N_{\tau}\right)$. To get the appropriate value for $\beta$ for other values of $N_{c}$, we demand that the 't Hooft coupling is kept fixed when adjusting $N_{c}$, meaning that $\beta_{\mathrm{SU}\left(N_{c}\right)}$ is determined by solving for it in the equation

$$
\frac{2 N_{c}^{2}}{\beta_{\mathrm{SU}\left(N_{c}\right)}}=\frac{18}{\beta_{\mathrm{SU}(3)}} .
$$

Lastly, to keep the Baryon mass constant when varying $a$, we adjust $\kappa$ according to the leading order expression equation (6.69). In this way we should get a rough estimate of the parameter space.

Figure 6.4 shows results of this strategy for the baryon density. As we can see, a continuum extrapolation should lead to a finite value. For $h_{1}<1$, this value should be smaller when $N_{c}$ is

\footnotetext{
${ }^{1}$ For more recent investigations of the $1+1$ dimensional case including chemical potential see [180].
} 

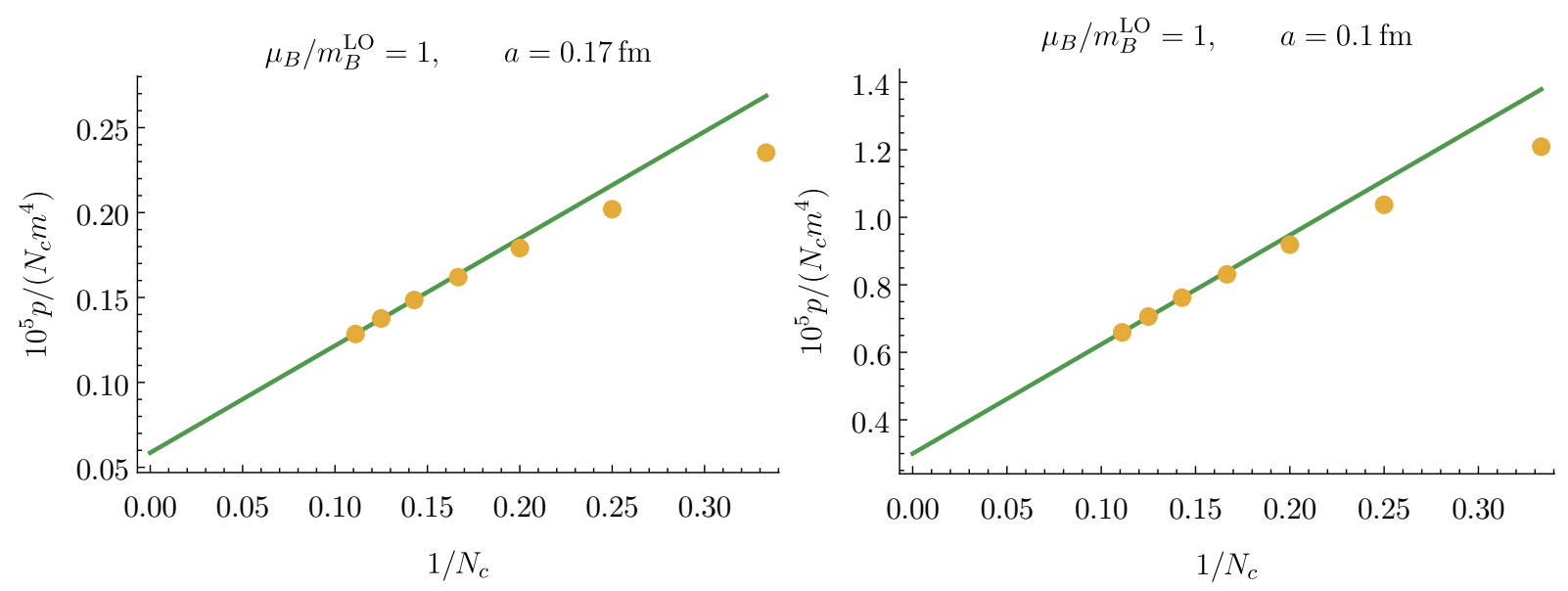

Figure 6.5: Adapted from [93]. The pressure at two different lattice spacings and $N_{c}$ values in the interval $[3,9]$. Its scaling is consistent with $p \sim N_{c}\left(1+\right.$ const. $\left.N_{c}^{-1}\right)$.

increased, and the situation reverses for $h_{1}>1$. In other words, the transition still seems to steepen with increasing $N_{c}$, even when the continuum is approached first.

Furthermore, in figure 6.5 we explored how the pressure behaves for different $N_{c}$ in the interval $[3,9]$ at values of the chemical potential where one has roughly half filling. At this value, observables should not yet be dominated by saturation. Still, the pressure to the calculated orders follows the scaling $\sim N_{c}$. As the $N_{c}$ values are still far away from the limit of infinite $N_{c}$, this is shown in the figure by the observation that the first subleading correction goes like $\sim N_{c}^{0}$. Furthermore, the figure also illustrates that these observations seem to be stable when the lattice distance is reduced. As an aside, we note that down to $N_{c} \approx 5$ the pressure is still very good approximated by the first subleading correction. In contrast to $[65,73]$, we are note able to explicitly take the continuum limit here. First, we have less orders available and furthermore the orders necessary to get stable results grows exponentially due to the difficulty in the transition region described in section 6.3.1. Consequently we cannot explicitly show the scaling of the pressure to be $p \sim N_{c}$ for $N_{c} \rightarrow \infty$ in the continuum and transition to be always first order. In any case, first order behavior is very difficult to describe using series expansion methods, which we used.

\subsubsection{The phase diagram for growing and large $N_{c}$}

Although we are not able to explicitly demonstrate that the onset transition to baryon matter at any temperature is first order for $N_{c} \rightarrow \infty$ in the continuum, we can nevertheless argue for it based on the qualitative features we observed, similar to section 5.1. As noted in that section, the scale for the critical endpoint can be estimated from the binding energy per baryon. In table 6.1, we observed that the interaction energy per baryon relative to the baryon mass scales as $\epsilon \sim$ const. Therefore, as expected in [173], the binding energy per baryon scales as $\sim N_{c}$ in units that are $N_{c}$-independent and the critical endpoint moves to ever higher temperatures when $N_{c}$ is increased. In contrast, in the large $N_{c}$ limit, the deconfinement temperature $T_{d}$ is independent of chemical potential and corresponds to that of Yang-Mills theory, for which lattice calculations indicate that for large $N_{c}$ the transition is within $\sim N_{c}^{-2}$ of the value at $N_{c}=3$ [189]. Consequently, in the limit $N_{c} \rightarrow \infty$, temperatures within $0<T<T_{d}$ are below the scale of the binding energy and the onset transition is always first order.

For $N_{c}=3$ and heavy quarks, the deconfinement transition is first order for $\mu=0$ and has a critical endpoint, see figure 2.1. In this case, one can think of the first order line parallel to the chemical potential axis in the large $N_{c}$ limit to appear gradually by straightening out the $N_{c}=3$ line 


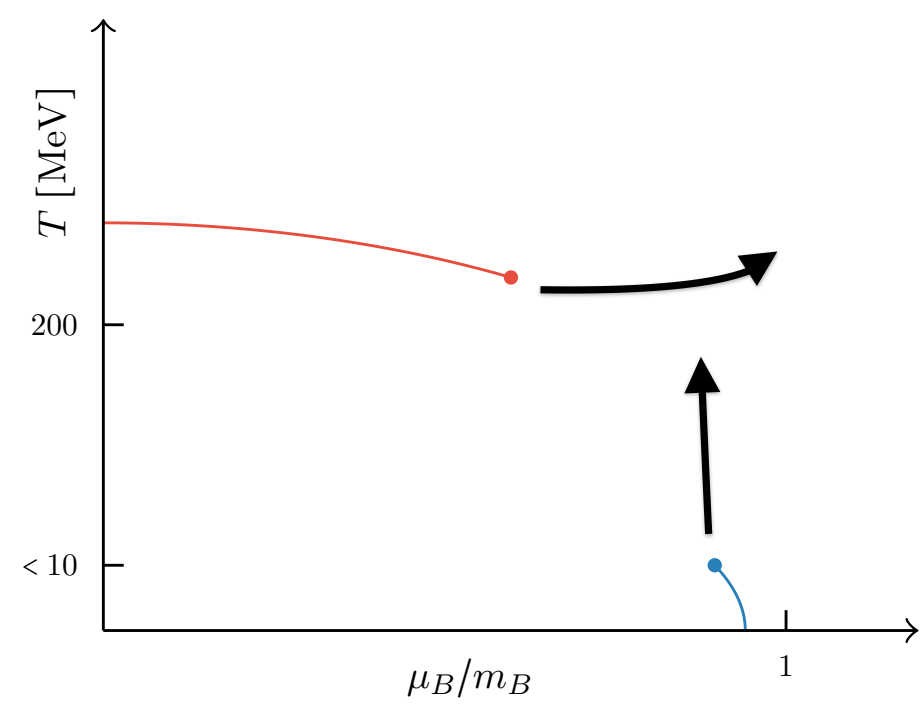

Figure 6.6: Take from [93]. Arrows indicate the gradual change of the phase transition lines with growing $N_{c}$.

and moving the endpoint to ever higher chemical potentials, as the quark loops have decreasing influence. Similarly, the endpoint for the onset transition moves up to higher temperatures, as explained above, until it hits another discontinuity. In this way, one observes how the rectangular phase diagram in figure 6.1 arises gradually from figure 2.1 by increasing $N_{c}$, as indicated in figure 6.6.

\subsubsection{Quarkyonic matter and lattice saturation}

After the onset, our scaling results are compatible with quarkyonic matter. Some care had to be taken to avoid artifacts due to lattice saturation. On the other hand, the situation with lattice saturation can be considered to be consistent with quarkyonic matter in the following way: As we explained in section 5.3, the relevant degrees of freedom for the lattice saturation density are those of quarks. At the same time, the onset transitions is related to the condensation of hadrons. We saw indications of the phenomenological properties leading to that conclusion in the effective theory in section 5.4, and simulations of the effective theory also support this conclusion as we discussed in the same section. Therefore, in a lattice filling up with baryon number one observes a rapid and smooth transition from baryon matter to matter whose thermodynamics is determined by quark degrees of freedom, just like it is the case with quarkyonic matter. When the lattice is made finer, the lattice saturation level increases when expressed in physical units, as well as the chemical potential that is necessary to saturate the lattice. In the continuum, one will then have a physical saturation effect at the level of the density of nuclear matter as explained in section 5.1. For the quarkyonic phase that smoothly introduces quark-like features for increasing density, the baryonic surface persists up to arbitrary finite quark chemical potential in the case of infinite $N_{c}$. For $N_{c}=3$, the interactions near the Fermi surface can be screened by quark loops for sufficiently large chemical potential and one has a transition to a regime which is completely perturbative in quarks and gluons. It is assumed that this transition is smooth, unless there is a deconfining transition that extends down to $T=0$ [173]. This question is very hard to approach using strong coupling methods. Likewise, whether there is also a chiral transition at larger chemical potential, possibly split from the deconfinement transition, cannot be decided within the current framework and should be investigated in an effective theory including chiral symmetry, such as [51]. 



\section{Conclusions and Research perspectives}

In this thesis we discussed both the derivation and evaluation of effective Polyakov theories of QCD at finite temperature and density.

The vertex-renormalized free graph expansion was applied to the SU(3) spin model to derive the free energy density to order 14 in the energy-like coupling and to order 60 in the magnetization-like coupling. In comparison to numerical results based on a flux representation, the series results to the current order are competitive for the equation of state up to a region relatively close to the phase transition but less precise for the critical couplings. Nevertheless, series methods have the advantage of being more flexible, as one can plot observables over a large range of values, once the series has been obtained. Furthermore, the evaluation of Polyakov loop theories via series expansion methods provides additional insights into the derivation of these theories, as we explained at the end of section 2.1.2. Additionally, in contrast to the flux representation, the free graph expansion has been generalized to $n$-point couplings [73]. One possible avenue of future research is to implement this generalization in our automatized generation of expansion coefficients. We remark however that in the case of logarithmic-resummed actions the free graph expansion is rather cumbersome or cannot be applied at all. It seems that the finite cluster method is the more sensible method in this case and we already showed in section 4.6.2 how to evaluate the logarithmic-resummed effective theory for $n$-point couplings using the finite cluster method. Therefore the automation of the finite cluster method in order to evaluate the effective theory seems more promising. A first step in this direction has been already made and we have implemented the finite cluster method for the effective theory that is used in [207], but the results have not yet been completely analyzed.

The finite cluster method can also be used for the derivation of the effective theory and naturally includes logarithmic resummations, as we showed in chapter 4. Large parts for the derivation of the effective theory following the strategy of that chapter have already been automated by computer implementations. Finishing these implementations would make it rather easy to obtain higher orders in the expansion. These higher order can be used to investigate some open questions from the main text. First, the possibility of invalid singularities in $h_{1}$, due to the occurrence of terms in the evaluation of the effective theory which correspond to partial contractions in the application of Wick's theoremshould be investigated.

In section 5.4 we saw that $\kappa^{2}$-results for the binding energy alone are not enough to distinguish the isospin and baryon case and only at $\kappa^{4}$ there hints of the correct behavior. Therefore higher order calculations are certainly of interest in that case, too. These should be complemented by simulations of the effective theory, where a first order transition has been seen for finite baryon density, to see if a second order transition line can be shown to exist for the effective theory with isospin chemical potential. Alternatively, the orders of the transitions can be studied using mean field methods, which is currently in progress.

Mean field methods are also attractive in the large $N_{c}$ limit, where they are believed to be exact. Redoing the calculations of [179], which concern the spin model for large $N_{c}$, while keeping the difference between $\mathrm{U}\left(N_{c}\right)$ and $\mathrm{SU}\left(N_{c}\right)$ in mind as suggested by [180] should be a valuable first step into the application of mean field theory for large $N_{c}$ to the actions we are using.

Concerning the large $N_{c}$ results in this thesis, we have shown that the pressure scales as $p \sim N_{c}$ beyond the onset transition for all available orders (up to including $\kappa^{4}$ ) in the hopping expansion and that the onset transition becomes first-order for large $N_{c}$ when the lattice spacing is kept fixed. Furthermore this behavior was observed to be stable under the inclusion of the leading gauge 
corrections. However, we also observed that the continuum limit should be taken before the large $N_{c}$ limit, which complicates matters. So far we have been unable to reach unambiguous results for continuum physics, but have at least been able to show that our findings are stable in the correct order of the limits in the range of lattice spacings and $N_{c}$-values that are under control. In this way our results are consistent with the prediction of quarkyonic matter and the large $N_{c}$ phase diagram from [173]. If our results indeed generalize to all orders in the hopping expansion and are stable under the correct order of the limits, then they would be genuine features of QCD also for physical quark masses. For the case $N_{c}=3$ there will be no phase transition to a quarkyonic phase and the onset transition which ends at a temperature of $\mathcal{O}(10 \mathrm{MeV})$ marks the condensation of baryons. Nevertheless, this is consistent with the shell picture of quarkyonic matter in momentum space, in which the formation of an inner sphere of quark matter in the Fermi sea for increasing density would allow for a smooth transition to matter which has mainly quark-like properties, up to the point where it can be described exclusively in terms of perturbative quarks and gluons. This is possible for $N_{c}=3$ as screening due to quark loops is not suppressed. If this is marked by an deconfinement transition or whether there is in addition a chiral transition cannot be decided based on our results.

In this thesis we did not discuss the combinatorics on how the gauge corrections to our results are obtained and simply used previous results. It would be interesting to study how to include and derive gauge corrections in the best way in the framework of the finite cluster method. As the fundamental geometry of the lattice for the gauge part of the action is the plaquette, it might be advisable to use the finite lattice method which is closely related to the finite cluster method, see [208] for an introduction and application to strong coupling expansions. As a notable achievement of the finite lattice method, stressing the power of this method, we mention the derivation of the high-temperature series expansion of the free energy density of the simple cubic Ising model to 50th order in the inverse temperature [209].

Certainly, at some point the combinatorial complexity in all methods will grow beyond what can be managed even with automated methods. Besides finding further analytic resummations, higher order contributions can also be incorporated using the non-perturbative methods mentioned in section 2.1.2 Most interestingly from the standpoint of this thesis, the finite cluster or lattice methods can be used to modify the strategy applied in [92]. There, the authors used two possible forms of the effective action. One form has couplings which are easily determined numerically by the computation of expectation values of $n$-point functions of Polyakov loops in the full theory. This form has the downside that its truncated version only includes interactions at short ranges. The second form of the effective theory used in [92] includes long range interactions, however the couplings cannot be directly determined using numerical methods. Therefore, one perturbatively determines the same expectation values that were used to determine the couplings of the first form using the second form of the effective action and in this way relates the two sets of couplings. Inverting these relations then determines the couplings in the second, more interesting form, that can also resolve phase transitions. Note, however, that the perturbative determination introduces additional systematic errors and becomes cumbersome very fast, when multiple couplings are used. This is where the finite cluster (and lattice) methods may introduce and interesting alternative: due to the form the finite cluster contributions enter the action, see section 4.4.4, one can use the first form of the effective theory to determine couplings on finite clusters by simulations on these clusters and the resulting action, which contains a translation of the finite clusters over the whole lattice, still resolves long range interactions.

So far, the methods in [92] have only been applied to pure gauge theory. Extending this approach to the fermionic sector is a further interesting prospect. It should be possible to avoid the sign problem in doing so, similar to [90], as we know how chemical potential enters the effective theory: by multiplying $h_{1}^{(f)}$ by a factor of $e^{a \mu^{(f)}}$ and $\bar{h}_{1}^{(f)}$ by a factor of $e^{-\mu^{(f)}}$. Therefore, simulations 
at $\mu=0$ should suffice in this case. Potential ambiguities arising from the fact that traces of higher powers of $\mathrm{SU}(3)$-matrices can be reexpressed in the way explained in section 4.6.3 should be resolvable by employing simulations at imaginary chemical potential (which are also free of a sign problem) $[72,89]$. Simulations at imaginary chemical potential can also increase the precision of the approach.

All in all, there are several interesting possibilities for future investigations. 



\section{A Notations}

We collect here some notations which have been defined when they were used the first time in this thesis, but may be useful whenever the thesis is not read from start to finish.

- We denote by $\mathbb{N}$ all natural numbers including 0 . For $\mathbb{N} \backslash\{0\}$ we use the notation $\mathbb{N}^{*}$.

- For a finite set $M,|M|$ denotes the number of elements in that set.

- If $S$ is a logical statement, then $\delta(S)$ is 1 if $S$ is true and 0 otherwise, the inspiration for doing so is $[96,210]$. This means the usual Kronecker delta can be written as $\delta_{i j}=\delta(i=j)$, but we employ the usual notation in this case.

- $\mathcal{S}_{k}(M)$ is the set of all subsets of $M$ with $k$ elements.

- If $M$ is a set of polymers endowed with a binary symmetric relation which decides if two polymers are to be regarded linked or disjoint, then $\gamma_{i} \cap \gamma_{j} \neq \emptyset$ denotes that the polymers are linked and $\gamma_{i} \cap \gamma_{j}=\emptyset$ when they are disjoint.

- For the same set of polymers $M, \mathcal{D}_{k}(M)$ is $\mathcal{S}_{k}(M)$ with only those subsets of $M$ that have pairwise disjoint polymers.

- For the same set of polymers $M, \mathcal{C}_{k}(M)$ is $\mathcal{S}_{k}(M)$ with only those subsets $C \subset M$ which have the property that for any pair $\gamma_{1}, \gamma_{2} \in C$ there is always a sequence of polymers $\gamma_{k_{1}}, \ldots, \gamma_{k_{m}}$ in $C$ that fulfills $\gamma_{k_{1}}=\gamma_{1}, \gamma_{k_{m}}=\gamma_{2}$ and $\gamma_{k_{i}} \cap \gamma_{k_{i+1}} \neq \emptyset$. 



\section{B Graphs}

We introduce some useful graph-theoretic notions used in the main text. Our notation and definitions are based on a combination of those introduced in [124, 126, 133, 211-213]. A graph consists of two finite sets $V_{G}$ of vertices and $E_{G}$ of edges together with an edge-to-endpoint function $\sigma_{G}$. The edge-to-endpoint function has the domain $E_{G}$. For an undirected graph, the edge-to-endpoint function is a mapping

$$
\sigma_{G}: E_{G} \rightarrow V_{G} \cup \mathcal{S}_{2}\left(V_{G}\right)
$$

and for a directed graph it is a mapping

$$
\sigma_{G}: E_{G} \rightarrow V_{G} \times V_{G}
$$

For a directed graph, one can define two further mappings

$$
\begin{aligned}
i_{G}: E_{G} \rightarrow V_{G}, \\
t_{G}: E_{G} \rightarrow V_{G},
\end{aligned}
$$

such that $\sigma_{G}(e)=\left(i_{G}(e), t_{G}(e)\right)$ for all $e \in E_{G}$. In a pictorial representation of graphs, the vertices are points which are linked by edges and the edge-to-endpoint function assigns to edges the vertices they are linking. Two vertices which are connected by an edge are called adjacent. For directed graphs, the edges are illustrated as arrows with their tail at $i(e)$ and head at $t(e)$.

A graph has a loop if it has an edge that links a vertex with itself, i.e. there is an $e \in E_{G}$ such that $\sigma_{G}(e) \in V_{G}$ for an undirected graph or $\sigma_{G}(e) \in(v, v), v \in V_{G}$ for a directed graph. An undirected graph without loops and where every pair of vertices is at most linked by one edge is called simple graph. If we want to stress that a pair of vertices can be connected by multiple edges we sometimes say that the graph is a multigraph. Unless explicitly said, we assume a graph to be free of loops.

An enumeration of the vertices of a graph $G$ with $n$ vertices corresponds to an invertible map $\pi:\{1,2, \ldots, n\} \rightarrow V_{G}$. Given an enumeration, one can define the adjacency matrix of an undirected graph as the matrix $A=\left(a_{i, j}\right)_{n \times n}$ with entries

$$
a_{i, j}= \begin{cases}\left|\sigma_{G}^{-1}(\{\{\pi(i), \pi(j)\}\})\right|, & \text { if } i \neq j \\ \sigma_{G}^{-1}(\{\pi(i)\}) \mid, & \text { if } i=j,\end{cases}
$$

and for a directed graph the entries read

$$
a_{i, j}=\left|\sigma_{G}^{-1}(\{(\pi(i), \pi(j))\})\right| .
$$

In other words, after labeling the vertices of the graph with the numbers 1 to $n$, the entry $a_{i j}$ of the adjacency matrix indicates the number of times the vertex labeled by $i$ is linked to the vertex labeled by $j$ in case of an undirected graph. For directed graphs, the entry counts the number of directed edges with tail at $i$ and head at $j$.

A subgraph $g$ of another graph $G$ is defined to be a graph whose vertices and edges are a subsets of the vertices and edges of $G$ and the edge-to-endpoint function of $g$ is the restriction of $\sigma_{G}$ to $E_{g}$. The union ${ }^{1} G_{1} \cup G_{2}$ of two graphs $G_{1}$ and $G_{2}$ is the graph with vertices $V_{G_{1}} \cup V_{G_{2}}$, edges $E_{G_{1}} \cup E_{G_{2}}$ 


\section{$B$ Graphs}

and edge-to-endpoint function $\sigma_{G_{1} \cup G_{2}}(e)=\sigma_{G_{1 / 2}}(e)$ if $e \in E_{G_{1 / 2}}$. We note that we assume that the two graphs are assumed to have consistent edge-to-endpoint functions on $E_{G_{1}} \cap E_{G_{2}}$.

The degree $n(v)$ of a vertex $v$ of a graph $G$ is the number of edges $e$ with the property that $v \in \sigma_{G}(e)$ for an undirected graph or $v \in\left\{i_{G}(e), t_{G}(e)\right\}$ for a directed graph. In the case of a directed graph we can furthermore define the in-degree $n_{\text {in }}(v)$ by the number of edges $e$ with $t(b)=v$ and the out-degree $n_{\text {out }}(v)$ is defined analogously. Of course, $n(v)=n_{\text {in }}(v)+n_{\text {out }}(v)$. A vertex with $n(v)=0$ is called an isolated vertex.

In some cases one assigns a subset of the vertices to be rooted/external vertices $R_{G}$, which are distinguished from the internal vertices $I_{G}, V_{G}=R_{G} \cup I_{G}$. Whenever it is not explicitly mentioned, we assume a graph to consist of internal vertices only. The external vertices are special in the way they enter the definition of a graph isomorphism. We say that two graphs $G_{1}$ and $G_{2}$ are isomorphic if there are two mappings

$$
\begin{aligned}
& \varphi: V_{G_{1}} \rightarrow V_{G_{2}}, \\
& \lambda: E_{G_{1}} \rightarrow E_{G_{2}}
\end{aligned}
$$

with the properties that

(a) they are both bijective,

(b) $\left.\varphi\right|_{R_{G}}=\left.\mathrm{id}\right|_{R_{G}}$,

(c) $\varphi\left(\sigma_{G_{1}}(e)\right)=\sigma_{G_{2}}(\lambda(e))$ for an undirected graph and $\left(\varphi \circ i_{G_{1}}(e), \varphi \circ t_{G_{1}}(e)\right)=\left(i_{G_{2}} \circ \lambda(e), t_{G_{2}} \circ\right.$ $\lambda(e))$ for a directed graph for all $e \in E(G)$.

Counting the number of elements in the automorphism group of a graph $G$ gives the symmetry number $S(G)$ of a graph.

Two vertices $v_{1}$ and $v_{2}$ are defined to be connected if there is a sequence of vertices $w_{1}, \ldots, w_{n}$ with the property that $w_{k}$ is adjacent to $w_{k+1}$ for $k<n$ and $w_{1}=v_{1}$ and $w_{n}=v_{2}$. A graph is said to be a connected graph if its vertices are pairwise connected.

$G \backslash v$ denotes the graph that is obtained by deleting the vertex $v$, which means removing $v$ from $V_{G}$, all edges $e$ from $E_{G}$ that fulfill $v \in \sigma_{G}(e)$ for an undirected graph or $v \in\left\{i_{G}(e), t_{G}(e)\right\}$ for a directed graph and restricting the edge-to-endpoint function appropriately. If $G$ is a connected graph, but $G \backslash v$ is not, then $v$ is said to be an articulation point of $G$. Graphs with no articulation point are called 1-irreducible graphs.

Lastly, a 1-insertion is a graph with one external vertex which has the property that deleting the external vertex leaves the graph connected.

\footnotetext{
${ }^{1}$ In the literature, graph unions are often defined as disjoint unions, this is not the way we define graph unions, as our definition is more convenient in our case.
} 


\section{The $\mathrm{SU}(N)$ one link integral}

In [214], the integral

$$
\int_{\mathrm{U}(N)} \mathrm{d} U e^{\lambda \operatorname{tr}\left(J U+U^{\dagger} K\right)}
$$

with $J$ and $K$ elements of the general linear group $\mathrm{GL}(N)$, was determined in terms of the characters of $\mathrm{GL}(N)$. We will use the same strategy to determine the integral for $\mathrm{SU}(N)$. For $\mathrm{SU}(3)$, the integral was computed in [102] and the $\mathrm{SU}(N)$ case is also treated inter alia in [55, 103]. From section 6.1 .1 we already know how the integrals over $\mathrm{SU}(N)$ are related to integrals over $\mathrm{U}(N)$ in terms of eigenvalues of the matrices. For general integrals this implies

$$
\int_{\mathrm{SU}(N)} \mathrm{d} U f(U)=\sum_{q=-\infty}^{\infty} \int_{\mathrm{U}(N)} \mathrm{d} U \operatorname{det}(U)^{q} f(U)
$$

where $f: \mathrm{U}(N) \rightarrow \mathbb{C}$ is not necessarily a class function.

This suggests to study the integral

$$
I_{q}=\int_{\mathrm{U}(N)} \mathrm{d} U \operatorname{det}(U)^{q} e^{\lambda \operatorname{tr}\left(J U+U^{\dagger} K\right)} .
$$

First, we will investigate the case $q \geq 0$. Making use of the multiplicative property of the determinant we obtain in this case

$$
\begin{aligned}
I_{q} & =\int_{\mathrm{U}(N)} \mathrm{d} U \operatorname{det}(U)^{q} e^{\lambda \operatorname{tr}\left(J U+U^{\dagger} K\right)} \\
& =\operatorname{det}(K)^{q} \int_{\mathrm{U}(N)} \mathrm{d} U \frac{1}{\operatorname{det}\left(U^{\dagger} K\right)^{q}} e^{\lambda \operatorname{tr}\left(J U+U^{\dagger} K\right)}
\end{aligned}
$$

The integrand can be written as the product of two class functions of $\operatorname{GL}(N)$ (see section 6.1.1 for a definition of class functions), which means they have an expansion in terms of the characters $\chi_{r}$ of $\operatorname{GL}(N)$ :

$$
\begin{aligned}
e^{\lambda \operatorname{tr}(J U)} & =\sum_{r} a_{r}(\lambda) \chi_{r}(J U), \\
\frac{1}{\operatorname{det}\left(U^{\dagger} K\right)} e^{\lambda \operatorname{tr}\left(U^{\dagger} K\right)} & =\sum_{r} b_{r, q}(\lambda) \chi_{r}(J U),
\end{aligned}
$$

where the sums go over all irreducible representations of $\mathrm{GL}(N)$.

Then, we can make use of the orthogonality of the characters

$$
\int_{\mathrm{U}(N)} \mathrm{d} U \chi_{r}(J U) \chi_{r^{\prime}}\left(U^{\dagger} K\right)=\delta_{r r^{\prime}} \frac{\chi_{r}(J K)}{d_{r}}
$$

where $d_{r}$ denotes the dimension of the representation. The orthogonality holds here despite the fact that we integrate over $\mathrm{U}(N)$, but the characters are those of $\operatorname{GL}(N)$. This is due to the fact that the characters are polynomials of the components $a_{i j}$ of the group element $A \in \mathrm{GL}(N)$ and 
the algebraic irrelevance of the unitary restriction [200]. Consequently, we obtain

$$
I_{q}=\operatorname{det}(K)^{q} \sum_{r} a_{r}(\lambda) b_{r}(\lambda) \frac{\chi_{r}(J K)}{d_{r}} .
$$

Next, we need to determine $a_{r}$ and $b_{r}$.

Again, due to the algebraic irrelevance of the unitary restriction, the character expansion coefficients are the same for $\mathrm{U}(N)$ and $\mathrm{GL}(N)$ and $a_{r}(\lambda)$ can be computed using

$$
a_{r}(\lambda)=\int_{\mathrm{U}(N)} \mathrm{d} U \chi_{r}\left(U^{\dagger}\right) e^{\lambda \operatorname{tr}(U)}
$$

The representation $r$ of $\mathrm{U}(N)$ can be labeled by $N$ integers $r=\left(n_{1}, n_{2}, \ldots, n_{N}\right)$, with $n_{1} \geq n_{2} \geq$ $\cdots \geq n_{N}$. The corresponding $a_{r}$ has been obtained in [215] and reads

$$
a_{\left(n_{1}, \ldots, n_{N}\right)}(\lambda)=\frac{\lambda^{\sum_{l=1}^{N} n_{l}}}{\prod_{i=1}^{N} n_{i} !} \prod_{1 \leq i<j \leq N}\left(1-\frac{n_{j}}{n_{i}+j-i}\right) .
$$

Using the same procedure (or alternatively the approach suggested in [216]) for $b_{r}(\lambda)$ one gets

$$
b_{\left(n_{1}, \ldots, n_{N}\right), q}(\lambda)=\frac{\lambda^{q N+\sum_{l=1}^{N} n_{l}}}{\prod_{i=1}^{N}\left(n_{i}+k\right) !} \prod_{1 \leq i<j \leq N}\left(1-\frac{n_{j}+k}{n_{i}+k+j-i}\right) .
$$

In the case $q<0$ we define $p=-q>0$ and write

$$
I_{q}=\operatorname{det}(J)^{p} \int_{\mathrm{U}(N)} \operatorname{det}(U) \frac{1}{\operatorname{det}(J U)^{p}} e^{\lambda \operatorname{tr}(J U+U K)}
$$

This integral can be evaluated in the same way as the previous case and in total we obtain

$$
\int_{\mathrm{SU}(N)} \mathrm{d} U e^{\lambda \operatorname{tr}\left(J U+U^{\dagger} K\right)}=\sum_{q=0}^{\infty}\left(1-\frac{1}{2} \delta_{k 0}\right)\left(\operatorname{det}(J)^{q}+\operatorname{det}(K)^{q}\right) \sum_{r} a_{r}(\lambda) b_{r, q}(\lambda) \frac{\chi_{r}(J K)}{d_{r}} .
$$

The dimension of the representations can be computed using

$$
d_{n_{1}, \ldots, n_{N}}=\prod_{1 \leq i<j \leq N}\left(1+\frac{n_{i}-n_{j}}{j-i}\right)
$$

and the characters are expressed in terms of traces in the following way [217]:

$$
\chi_{\left(n_{1}, \ldots, n_{N}\right)}=\operatorname{det}_{1 \leq i, j \leq N}\left(\chi_{n_{j}+i-j}\right) .
$$

here $\chi_{n}=0$ for $n \leq 0$ and otherwise for a matrix $H \in \mathrm{GL}(N)$

$$
\chi_{n}(H)=\sum_{k_{1}, \ldots, k_{N} \in \mathbb{N}} \frac{\delta\left(\sum_{l=1}^{N} l k_{l}=N\right)}{\prod_{l=1}^{N} k_{l} !} \prod_{l=1}^{N}\left(\frac{\operatorname{tr}(H)}{l}\right)^{k_{l}} .
$$

Finally, using these equations we give the characters necessary for the $\mathcal{O}\left(\kappa^{4}\right)$ calculation together with their dimension for $N \geq 2$

$$
\chi_{(1,0, \ldots, 0)}(H)=\operatorname{tr}(H)
$$




$$
\begin{aligned}
d_{(1,0, \ldots, 0)} & =N, \\
\chi_{(2,0, \ldots, 0)}(H) & =\frac{1}{2}\left[\operatorname{tr}(H)^{2}+\operatorname{tr}\left(H^{2}\right)\right], \\
d_{(2,0, \ldots, 0)} & =\frac{N(N+1)}{2}, \\
\chi_{(1,1,0, \ldots, 0)}(H) & =\frac{1}{2}\left[\operatorname{tr}(H)^{2}-\operatorname{tr}\left(H^{2}\right)\right], \\
d_{(1,1,0, \ldots, 0)} & =\frac{N(N-1)}{2} .
\end{aligned}
$$





\section{A determinant evaluation}

In this chapter we derive a formula for a determinant that is used in the main text. Employing techniques described in [218], we will obtain an expression for

$$
\operatorname{det}_{1 \leq i, j \leq N}\left(\left(\begin{array}{c}
A \\
L_{i}-j
\end{array}\right)\right) .
$$

Note that we are a bit lax concerning possibly vanishing denominators here, the calculations can in principle be made more rigorous by using generalizations of binomial coefficients and factorials to real values and introducing appropriate limits.

The general strategy we use here is to bring the determinant to a form where lemma 3 in [218] can be used, which states that

$$
\operatorname{det}_{1 \leq i, j \leq N}\left(\prod_{k=j+1}^{N}\left(X_{i}+A_{k}\right) \prod_{k=2}^{j}\left(X_{i}+B_{k}\right)\right)=\prod_{1 \leq i<j \leq N}\left(X_{i}-X_{j}\right) \prod_{2 \leq i \leq j \leq n}\left(B_{i}-A_{j}\right) .
$$

To apply this lemma, we have to get rid of the denominator inside the determinant. To this end, we note that in the matrix element

$$
\left(\begin{array}{c}
A \\
L_{i}-j
\end{array}\right)=\frac{A !}{\left(L_{i}-j\right) !\left(A-L_{i}+j\right) !},
$$

the first factor in the denominator can be at most $L_{i}-1$ in the $i^{\text {th }}$ row and the second cannot exceed $A-L_{i}+N$. Pulling these factors and the numerator out, one obtains

$$
\begin{aligned}
\left(\begin{array}{c}
A \\
L_{i}-j
\end{array}\right)= & \frac{A !}{\left(L_{i}-1\right) !\left(A-L_{i}+N\right) !} \frac{\left(L_{i}-1\right) !\left(A-L_{i}+N\right) !}{\left(L_{i}-j\right) !\left(A-L_{i}+j\right) !} \\
= & \frac{A !}{\left(L_{i}-1\right) !\left(A-L_{i}+N\right) !}\left(L_{i}-1\right)\left(L_{i}-2\right) \cdots\left(L_{i}-j+1\right) \\
& \times\left(A-L_{i}+N\right)\left(A-L_{i}+(N-1)\right) \cdots\left(A-L_{i}+j+1\right) \\
= & (-1)^{N-j} \frac{A !}{\left(L_{i}-1\right) !\left(A-L_{i}+N\right) !} \prod_{k=2}^{j}\left(L_{i}-k+1\right) \prod_{k=j+1}^{N}\left(L_{i}-k-A\right) .
\end{aligned}
$$

Using the multilinearity of the determinant then results in

$$
\begin{aligned}
\operatorname{det}_{1 \leq i, j \leq N}\left(\left(\begin{array}{c}
A \\
L_{i}-j
\end{array}\right)\right)= & (-1)^{\sum_{j=1}^{N}(N-j)} \prod_{i=1}^{N} \frac{A !}{\left(L_{i}-1\right) !\left(A-L_{i}+N\right) !} \\
& \times \operatorname{det}_{1 \leq i, j \leq N}\left(\prod_{k=j+1}^{N}\left(L_{i}-k-A\right) \prod_{k=2}^{j}\left(L_{i}-k+1\right)\right) .
\end{aligned}
$$




\section{$D$ A determinant evaluation}

where one can use

$$
\sum_{j=1}^{N}(N-j)=\frac{N(N-1)}{2}=\left(\begin{array}{c}
N \\
2
\end{array}\right)
$$

for the prefactor.

To apply equation (D.2), we make the identifications $X_{i}=L_{i}, A_{k}=-k-A$ and $B_{k}=-k+1$ to obtain

$$
\begin{aligned}
\operatorname{det}_{1 \leq i, j \leq N}\left(\left(\begin{array}{c}
A \\
L_{i}-j
\end{array}\right)\right)= & (-1)^{\left(\begin{array}{c}
N \\
2
\end{array}\right)} \prod_{i=1}^{N} \frac{A !}{\left(L_{i}-1\right) !\left(A-L_{i}+N\right) !} \\
& \times \prod_{1 \leq i<j \leq N}\left(L_{i}-L_{j}\right) \prod_{2 \leq i \leq j \leq N}(A+1+j-i) .
\end{aligned}
$$

The last product can be rewritten:

$$
\begin{aligned}
\prod_{2 \leq i \leq j \leq N}(A+1+j-i) & =\prod_{j=2}^{N} \prod_{i=2}^{j}(A+1+j-i) \\
& =\prod_{j=2}^{N}(A+j-1) \cdot(A+j-2) \cdots(A+1) \\
& =\prod_{j=2}^{N} \frac{(A+j-1) !}{A !} \\
& =\prod_{i=1}^{N} \frac{(A+i-1) !}{A !} \\
& =\prod_{i=1}^{N} \frac{(A+N-i) !}{A !}
\end{aligned}
$$

We furthermore note that there are $\left(\begin{array}{c}N \\ 2\end{array}\right)$ possibilities for $i$ and $j$ to fulfill the condition $1 \leq i<j \leq N$ and therefore

$$
(-1)^{\left(\begin{array}{l}
N \\
2
\end{array}\right)} \prod_{1 \leq i<j \leq N}\left(L_{i}-L_{j}\right)=\prod_{1 \leq i<j \leq N}\left(L_{j}-L_{i}\right) .
$$

Putting this together, we obtain

$$
\begin{aligned}
\operatorname{det}_{1 \leq i, j \leq N}\left(\left(\begin{array}{c}
A \\
L_{i}-j
\end{array}\right)\right) & =\prod_{i=1}^{N} \frac{(A+N-i) !}{\left(L_{i}-1\right) !\left(A+N-L_{i}\right) !} \prod_{1 \leq i<j \leq N}\left(L_{j}-L_{i}\right) \\
& =\prod_{i=1}^{N} \frac{(A+N-i) \frac{L_{i}-i}{}}{\left(L_{i}-1\right) !} \prod_{1 \leq i<j \leq N}\left(L_{j}-L_{i}\right),
\end{aligned}
$$

where we have introduced underline notation for the falling factorials $k>0$

$$
\begin{aligned}
n^{\underline{k}} & =n \cdot(n-1) \cdots(n-k+1) \\
& =\frac{n !}{(n-k) !} .
\end{aligned}
$$

Note that the last equation also makes sense for $k \leq 0$ and taking the $\Gamma$-function as the generalization of the factorial it is also is consistent with the previous to last equation for the cases $k>n$ 
and $n<0$ in a sense where appropriate limits have been taken. For $k>0$ we therefore also have

$$
n \frac{-k}{(n+1)^{\bar{k}}},
$$

introducing overline notation for rising factorials

$$
n^{\bar{k}}=n \cdot(n+1) \cdots(n+k-1) .
$$

Up to a relative sign, equation (D.17) is a special case of equation (3.13) in [218] which has been proven in [219]. Indeed, equation (3.13) from [218] can be used to evaluate the determinant in (6.41), however, equation (D.17) can be applied to the calculation of determinants which come up when going beyond the static determinant. 



\section{Bibliography}

[1] ATLAS Collaboration, G. Aad, T. Abajyan, B. Abbott, et al., "Observation of a new particle in the search for the standard model higgs boson with the ATLAS detector at the LHC", Physics Letters B, vol. 716, no. 1, pp. 1-29, Sep. 17, 2012. DOI: 10.1016/j . physletb. 2012. 08.020. arXiv: 1207.7214 [hep-ex].

[2] CMS Collaboration, S. Chatrchyan, V. Khachatryan, A. M. Sirunyan, et al., "Observation of a new boson at a mass of $125 \mathrm{GeV}$ with the CMS experiment at the LHC", Physics Letters $B$, vol. 716 , no. 1 , pp. 30-61, Sep. 17, 2012. DOI: 10.1016/j . physletb. 2012.08.021. arXiv: 1207.7235 [hep-ex].

[3] J. Greensite, An Introduction to the Confinement Problem, 2nd ed., ser. Lecture Notes in Physics 972. Springer International Publishing, 2020. DOI: 10.1007/978-3-030-51563-8.

[4] K. G. Wilson, "Confinement of quarks", Physical Review D, vol. 10, no. 8, pp. 2445-2459, Oct. 15, 1974. DOI: 10.1103/physrevd.10.2445.

[5] K. Osterwalder and E. Seiler, "Gauge field theories on a lattice", Annals of Physics, vol. 110, no. 2, pp. 440-471, Feb. 1978. DOI: 10.1016/0003-4916(78)90039-8.

[6] H. B. Nielsen and M. Ninomiya, "Absence of neutrinos on a lattice: (I). proof by homotopy theory", Nuclear Physics B, vol. 185, no. 1, pp. 20-40, Jul. 13, 1981. DOI: 10.1016/05503213(81)90361-8.

[7] H. B. Nielsen and M. Ninomiya, "Absence of neutrinos on a lattice: (II). intuitive topological proof", Nuclear Physics B, vol. 193, no. 1, pp. 173-194, Dec. 21, 1981. DOI: 10.1016/05503213 (81) 90524-1.

[8] H. B. Nielsen and M. Ninomiya, "A no-go theorem for regularizing chiral fermions", Physics Letters B, vol. 105, no. 2-3, pp. 219-223, Oct. 1, 1981. DOI: 10.1016/0370-2693(81)910261.

[9] Budapest-Marseille-Wuppertal collaboration, S. Borsányi, S. Dürr, Z. Fodor, C. Hoelbling, S. D. Katz, S. Krieg, T. Kurth, L. Lellouch, T. Lippert, C. McNeile, and K. K. Szabó, "High-precision scale setting in lattice QCD", Journal of High Energy Physics, vol. 2012, no. 9, 10, Sep. 4, 2012. DOI: 10.1007/jhep09(2012)010. arXiv: 1203.4469 [hep-lat].

[10] R. Sommer, "Scale setting in lattice QCD", in Proceedings of 31st International Symposium on Lattice Field Theory - PoS (LATTICE 2013), vol. 187, Sissa Medialab, Apr. 2014, 015. DOI: $10.22323 / 1.187 .0015$. arXiv: 1401.3270 [hep-lat].

[11] S. Dürr, Z. Fodor, J. Frison, C. Hoelbling, R. Hoffmann, S. D. Katz, S. Krieg, T. Kurth, L. Lellouch, T. Lippert, K. K. Szabó, and G. Vulvert, "Ab initio determination of light hadron masses", Science, vol. 322, no. 5905, pp. 1224-1227, Nov. 2008. DOI: 10.1126 /science. 1163233. arXiv: 0906.3599 [hep-lat].

[12] Y. Aoki, G. Endrődi, Z. Fodor, S. D. Katz, and K. K. Szabó, "The order of the quantum chromodynamics transition predicted by the standard model of particle physics", Nature, vol. 443, no. 7112, pp. 675-678, Oct. 12, 2006. DOI: 10.1038/nature05120. arXiv: heplat/0611014. 
[13] Wuppertal-Budapest Collaboration, S. Borsányi, Z. Fodor, C. Hoelbling, S. D. Katz, S. Krieg, C. Ratti, and K. K. Szabó, "Is there still any $T_{c}$ mystery in lattice QCD? results with physical masses in the continuum limit III", Journal of High Energy Physics, vol. 2010, no. 9, Sep. 2010. DOI: 10.1007/jhep09(2010)073. arXiv: 1005.3508 [hep-lat].

[14] HotQCD Collaboration, A. Bazavov, T. Bhattacharya, M. Cheng, C. DeTar, H.-T. Ding, S. Gottlieb, R. Gupta, P. Hegde, U. M. Heller, F. Karsch, E. Laermann, L. Levkova, S. Mukherjee, P. Petreczky, C. Schmidt, R. A. Soltz, W. Soeldner, R. Sugar, D. Toussaint, W. Unger, and P. Vranas, "Chiral and deconfinement aspects of the QCD transition", Physical Review D, vol. 85, no. 5, 054503, Mar. 5, 2012. DoI: 10.1103/physrevd.85.054503. arXiv: 1111.1710 [hep-lat].

[15] C. Rohrhofer, Y. Aoki, G. Cossu, H. Fukaya, C. Gattringer, L. Y. Glozman, S. Hashimoto, C. B. Lang, and S. Prelovsek, "Symmetries of spatial meson correlators in high temperature QCD", Physical Review D, vol. 100, no. 1, p. 014502 , Jul. 2019. DOI: 10.1103/physrevd. 100.014502. arXiv: 1902.03191 [hep-lat].

[16] C. Rohrhofer, Y. Aoki, L. Y. Glozman, and S. Hashimoto, "Chiral-spin symmetry of the meson spectral function above $T_{c}$ ", Physics Letters B, vol. 802, 135245, Mar. 10, 2020. DOI: 10.1016/j.physletb.2020.135245. arXiv: 1909.00927 [hep-lat].

[17] O. Philipsen, "Constraining the phase diagram of QCD at finite temperature and density", in Proceedings of 37th International Symposium on Lattice Field Theory - PoS (LATTICE2019), vol. 363, Sissa Medialab, Aug. 27, 2020, 273. DOI: 10.22323/1.363.0273. arXiv: 1912.04827 [hep-lat].

[18] P. de Forcrand, "Simulating QCD at finite density", in Proceedings of The XXVII International Symposium on Lattice Field Theory - PoS (LAT2009), vol. 91, Sissa Medialab, Jun. 23, 2010, 010. DOI: 10.22323/1.091.0010. arXiv: 1005.0539 [hep-lat].

[19] C. Gattringer and K. Langfeld, "Approaches to the sign problem in lattice field theory", International Journal of Modern Physics A, vol. 31, no. 22, 1643007, Aug. 5, 2016. DOI: 10.1142/s0217751x16430077. arXiv: 1603.09517 [hep-lat].

[20] I. M. Barbour, S. E. Morrison, E. G. Klepfish, J. B. Kogut, and M.-P. Lombardo, "Results on finite density QCD", Nuclear Physics B - Proceedings Supplements, vol. 60, no. 1-2, pp. 220-233, Jan. 1998. DOI: 10.1016/s0920-5632 (97)00484-2. arXiv: hep-lat/9705042 [hep-lat].

[21] Z. Fodor and S. D. Katz, "A new method to study lattice QCD at finite temperature and chemical potential", Physics Letters B, vol. 534, no. 1-4, pp. 87-92, May 16, 2002. DoI: 10.1016/s0370-2693(02)01583-6. arXiv: hep-lat/0104001.

[22] Z. Fodor and S. D. Katz, "Lattice determination of the critical point of QCD at finite $T$ and 4", Journal of High Energy Physics, vol. 2002, no. 03, 014, Mar. 2002. DOI: 10.1088/11266708/2002/03/014. arXiv: hep-lat/0106002.

[23] F. Csikor, G. I. Egri, Z. Fodor, S. D. Katz, K. K. Szabó, and A. I. Toóth, "The QCD equation of state at finite T and $\mu "$, Nuclear Physics B - Proceedings Supplements, vol. 119, pp. 547-549, May 2003. DOI: 10.1016/s0920-5632(03)80453-x. arXiv: hep-lat/0209114.

[24] Z. Fodor and S. D. Katz, "Critical point of QCD at finite $T$ and $\mu$, lattice results for physical quark masses", Journal of High Energy Physics, vol. 2004, no. 04, 050, May 17, 2004. DOI: 10.1088/1126-6708/2004/04/050. arXiv: hep-lat/0402006.

[25] P. de Forcrand and O. Philipsen, "The QCD phase diagram for three degenerate flavors and small baryon density", Nuclear Physics B, vol. 673, no. 1-2, pp. 170-186, Nov. 24, 2003. DOI: 10.1016/j.nuclphysb.2003.09.005. arXiv: hep-lat/0307020. 
[26] M. D'Elia and M.-P. Lombardo, "Finite density QCD via an imaginary chemical potential", Physical Review D, vol. 67, no. 1, 014505, Jan. 28, 2003. DOI: 10.1103/physrevd.67.014505. arXiv: hep-lat/0209146 [hep-lat].

[27] O. Philipsen and C. Pinke, " $N_{f}=2$ qcd chiral phase transition with wilson fermions at zero and imaginary chemical potential", Physical Review D, vol. 93, no. 11, 114507, Jun. 9, 2016. DOI: 10.1103/physrevd.93.114507. arXiv: 1602.06129 [hep-lat].

[28] S. Borsanyi, Z. Fodor, J. N. Guenther, R. Kara, S. D. Katz, P. Parotto, A. Pasztor, C. Ratti, and K. K. Szabó, "QCD crossover at finite chemical potential from lattice simulations", Physical Review Letters, vol. 125, no. 5, 052001, Jul. 2020. DOI: 10.1103/physrevlett. 125.052001. arXiv: 2002.02821 [hep-lat].

[29] C. R. Allton, S. Ejiri, S. J. Hands, O. Kaczmarek, F. Karsch, E. Laermann, C. Schmidt, and L. Scorzato, "QCD thermal phase transition in the presence of a small chemical potential", Physical Review D, vol. 66, no. 7, 074507, Oct. 29, 2002. DOI: 10.1103/physrevd.66.074507. arXiv: hep-lat/0204010.

[30] C. R. Allton, M. Döring, S. Ejiri, S. J. Hands, O. Kaczmarek, F. Karsch, E. Laermann, and K. Redlich, "Thermodynamics of two flavor QCD to sixth order in quark chemical potential", Physical Review D, vol. 71, no. 5, 054508, Mar. 24, 2005. DOI: 10.1103/physrevd. 71 . 054508. arXiv: hep-lat/0501030.

[31] R. V. Gavai and S. Gupta, "QCD at finite chemical potential with six time slices", Physical Review D, vol. 78, no. 11, 114503, Dec. 15, 2008. DOI: 10.1103/physrevd.78.114503. arXiv: 0806.2233 [hep-lat].

[32] MILC Collaboration, S. Gottlieb, S. Basak, A. Bazavov, C. Bernard, C. DeTar, W. Freeman, U. M. Heller, J. E. Hetrick, J. Laiho, L. Levkova, J. Osborn, R. Sugar, and D. Toussaint, "QCD equation of state at non-zero chemical potential", in Proceedings of The XXVI International Symposium on Lattice Field Theory - PoS (LATTICE 2008), Sissa Medialab, Jun. 2009. DOI: 10.22323/1.066.0171. arXiv: 0910.0276 [hep-lat].

[33] O. Kaczmarek, F. Karsch, E. Laermann, C. Miao, S. Mukherjee, P. Petreczky, C. Schmidt, W. Soeldner, and W. Unger, "Phase boundary for the chiral transition in $(2+1)$-flavor QCD at small values of the chemical potential", Physical Review D, vol. 83, no. 1, 014504, Jan. 10, 2011. DOI: 10.1103/physrevd.83.014504. arXiv: 1011.3130 [hep-lat].

[34] HotQCD Collaboration, A. Bazavov, D. Bollweg, H.-T. Ding, P. Enns, J. Goswami, P. Hegde, O. Kaczmarek, F. Karsch, R. Larsen, S. Mukherjee, H. Ohno, P. Petreczky, C. Schmidt, S. Sharma, and P. Steinbrecher, "Skewness, kurtosis, and the fifth and sixth order cumulants of net baryon-number distributions from lattice QCD confront high-statistics STAR data", Physical Review D, vol. 101, no. 7, 074502, Apr. 3, 2020. DOI: 10.1103/physrevd.101. 074502. arXiv: 2001.08530 [hep-lat].

[35] G. Aarts, L. Bongiovanni, E. Seiler, D. Sexty, and I.-O. Stamatescu, "Controlling complex langevin dynamics at finite density", The European Physical Journal A, vol. 49, no. 7, 89, Jul. 17, 2013. DOI: 10.1140/epja/i2013-13089-4. arXiv: 1303.6425 [hep-lat].

[36] E. Seiler, D. Sexty, and I.-O. Stamatescu, "Gauge cooling in complex langevin for lattice QCD with heavy quarks", Physics Letters B, vol. 723, no. 1-3, pp. 213-216, Jun. 10, 2013. DOI: $10.1016 / j$.physletb.2013.04.062. arXiv: 1211.3709 [hep-lat] .

[37] D. Sexty, "Simulating full QCD at nonzero density using the complex langevin equation", Physics Letters B, vol. 729, pp. 108-111, Feb. 5, 2014. DOI: 10.1016/j .physletb. 2014. 01.019. arXiv: 1307.7748 [hep-lat]. 
[38] M. Cristoforetti, F. D. Renzo, and L. Scorzato, "New approach to the sign problem in quantum field theories: High density QCD on a Lefschetz thimble", Physical Review D, vol. 86, no. 7, 074506, Oct. 8, 2012. DOI: 10.1103/physrevd.86.074506. arXiv: 1205.3996 [hep-lat].

[39] A. Alexandru, G. Basar, P. F. Bedaque, G. W. Ridgway, and N. C. Warrington, "Sign problem and Monte Carlo calculations beyond Lefschetz thimbles", Journal of High Energy Physics, vol. 2016, no. 5, 53, May 10, 2016. DoI: 10.1007/jhep05(2016)053. arXiv: 1512. 08764 [hep-lat].

[40] L. Scorzato, "The Lefschetz thimble and the sign problem", in Proceedings of The 33rd International Symposium on Lattice Field Theory - PoS (LATTICE 2015), Sissa Medialab, Jul. 15, 2016. DOI: 10.22323/1.251.0016. arXiv: 1512.08039 [hep-lat] .

[41] C. Schmidt and F. Ziesché, "Simulating low dimensional QCD with Lefschetz thimbles", in Proceedings of 34th annual International Symposium on Lattice Field Theory - PoS (LATTICE2016), vol. 256, Sissa Medialab, Mar. 24, 2017, 076. DOI: 10.22323/1.256.0076. arXiv: 1701.08959 [hep-lat].

[42] F. D. Renzo and G. Eruzzi, "One-dimensional QCD in thimble regularization", Physical Review D, vol. 97 , no. 1, 014503, Jan. 12, 2018. DOI: 10.1103/physrevd.97.014503. arXiv: 1709.10468 [hep-lat].

[43] Z. Fodor, S. D. Katz, and C. Schmidt, "The density of states method at non-zero chemical potential", Journal of High Energy Physics, vol. 2007, no. 03, 121, Mar. 30, 2007. DOI: 10.1088/1126-6708/2007/03/121. arXiv: hep-lat/0701022.

[44] K. Langfeld, B. Lucini, and A. Rago, "Density of states in gauge theories", Physical Review Letters, vol. 109, no. 11, 111601, Sep. 11, 2012. DOI: 10.1103/physrevlett.109.111601. arXiv: 1204.3243 [hep-lat].

[45] G. Endrődi, Z. Fodor, S. D. Katz, D. Sexty, K. K. Szabó, and C. Török, "Applying constrained simulations for low temperature lattice QCD at finite baryon chemical potential", Physical Review D, vol. 98, no. 7, 074508, Oct. 15, 2018. DOI: 10.1103/physrevd.98. 074508. arXiv: 1807.08326 [hep-lat].

[46] A. Alexandru, M. Faber, I. Horváth, and K.-F. Liu, "Lattice QCD at finite density via a new canonical approach", Physical Review D, vol. 72, no. 11, 114513, Dec. 28, 2005. DOI: 10.1103/physrevd.72.114513. arXiv: hep-lat/0507020.

[47] S. Kratochvila and P. de Forcrand, "The canonical approach to finite density QCD", in Proceedings of XXIIIrd International Symposium on Lattice Field Theory - PoS (LAT2005), Sissa Medialab, Dec. 14, 2005. DOI: 10.22323/1.020.0167. arXiv: hep-lat/0509143.

[48] S. Ejiri, "Canonical partition function and finite density phase transition in lattice QCD", Physical Review D, vol. 78, no. 7, 074507, Oct. 8, 2008. DOI: 10.1103/physrevd.78. 074507. arXiv: 0804.3227 [hep-lat].

[49] P. Rossi and U. Wolff, "Lattice QCD with fermions at strong coupling: A dimer system", Nuclear Physics B, vol. 248, no. 1, pp. 105-122, Dec. 17, 1984. DOI: 10.1016/0550-3213 (84) 90589-3.

[50] J. W. Cherrington, J. D. Christensen, and I. Khavkine, "Dual computations of non-abelian Yang-Mills theories on the lattice", Physical Review D, vol. 76, no. 9, 094503, Nov. 5, 2007. DOI: 10.1103/physrevd.76.094503. arXiv: 0705.2629 [hep-lat].

[51] P. de Forcrand, J. Langelage, O. Philipsen, and W. Unger, "Lattice QCD phase diagram in and away from the strong coupling limit", Physical Review Letters, vol. 113, no. 15, 152002, Oct. 6, 2014. DOI: 10.1103/PhysRevLett.113.152002. arXiv: 1406.4397 [hep-lat]. 
[52] C. Gattringer, "New developments for lattice field theory at non-zero density", in Proceedings of 31st International Symposium on Lattice Field Theory - PoS (LATTICE 2013), Sissa Medialab, Apr. 28, 2014. DOI: 10.22323/1.187.0002. arXiv: 1401.7788 [hep-lat].

[53] C. Gattringer, T. Kloiber, and V. Sazonov, "Solving the sign problems of the massless lattice schwinger model with a dual formulation", Nuclear Physics B, vol. 897, pp. 732-748, Aug. 2015. DOI: 10.1016/j.nuclphysb.2015.06.017. arXiv: 1502.05479 [hep-lat].

[54] C. Gattringer and C. Marchis, "Abelian color cycles: A new approach to strong coupling expansion and dual representations for non-abelian lattice gauge theory", Nuclear Physics B, vol. 916, pp. 627-646, Mar. 2017. DOI: 10.1016/j . nuclphysb. 2017.01 .025 . arXiv: 1609.00124 [hep-lat].

[55] G. Gagliardi and W. Unger, "New dual representation for staggered lattice QCD", Physical Review D, vol. 101, no. 3, 034509, Feb. 12, 2020. DOI: 10.1103/physrevd.101.034509. arXiv: 1911.08389 [hep-lat].

[56] C. Ratti, "QCD at non-zero density and phenomenology", in Proceedings of The 36th Annual International Symposium on Lattice Field Theory - PoS (LATTICE2018), vol. 334, Sissa Medialab, May 29, 2019, 004. DOI: 10.22323/1.334.0004.

[57] C. S. Fischer, "QCD at finite temperature and chemical potential from Dyson-Schwinger equations", Progress in Particle and Nuclear Physics, vol. 105, pp. 1-60, Mar. 2019. DOI: 10.1016/j.ppnp.2019.01.002. arXiv: 1810.12938 [hep-ph].

[58] W.-j. Fu, J. M. Pawlowski, and F. Rennecke, "QCD phase structure at finite temperature and density", Physical Review D, vol. 101, no. 5, 054032, Mar. 24, 2020. DOI: $10.1103 /$ physrevd.101.054032. arXiv: 1909.02991 [hep-ph].

[59] F. Gao and J. M. Pawlowski, "QCD phase structure from functional methods", Physical Review D, vol. 102, no. 3, 034027, Aug. 25, 2020. DOI: 10.1103/physrevd.102. 034027. arXiv: 2002.07500 [hep-ph].

[60] M. G. Alford, A. Schmitt, K. Rajagopal, and T. Schäfer, "Color superconductivity in dense quark matter", Reviews of Modern Physics, vol. 80, no. 4, pp. 1455-1515, Nov. 11, 2008. DOI: $10.1103 /$ revmodphys.80.1455. arXiv: 0709.4635 [hep-ph].

[61] M. Fromm, J. Langelage, S. Lottini, and O. Philipsen, "The QCD deconfinement transition for heavy quarks and all baryon chemical potentials", Journal of High Energy Physics, vol. 2012, no. 01, 42, Jan. 11, 2012. DOI: 10.1007/JHEP01(2012) 042. arXiv: 1111. 4953 [hep-lat].

[62] F. Karsch and H. W. Wyld, "Complex langevin simulation of the $S U(3)$ spin model with nonzero chemical potential", Physical Review Letters, vol. 55, no. 21, pp. 2242-2245, Nov. 18, 1985. DOI: $10.1103 /$ physrevlett.55.2242.

[63] N. Bilić, H. Gausterer, and S. Sanielevici, "Complex langevin solution to an effective theory of lattice QCD", Physical Review D, vol. 37, no. 12, pp. 3684-3690, Jun. 15, 1988. DoI: 10.1103/physrevd.37.3684.

[64] G. Aarts and F. A. James, "Complex langevin dynamics in the $S U(3)$ spin model at nonzero chemical potential revisited", Journal of High Energy Physics, vol. 2012, no. 1, 118, Jan. 25, 2012. DOI: 10.1007/jhep01 (2012)118. arXiv: 1112.4655 [hep-lat].

[65] J. Langelage, M. Neuman, and O. Philipsen, "Heavy dense QCD and nuclear matter from an effective lattice theory", Journal of High Energy Physics, vol. 2014, no. 09, 131, Sep. 23, 2014. DOI: 10.1007/JHEP09 (2014)131. arXiv: 1403.4162 [hep-lat]. 
[66] J. Greensite, "Comparison of complex langevin and mean field methods applied to effective polyakov line models", Physical Review D, vol. 90, no. 11, 114507, Dec. 24, 2014. DoI: 10.1103/physrevd.90.114507. arXiv: 1406.4558 [hep-lat].

[67] C. Gattringer, "Flux representation of an effective polyakov loop model for QCD thermodynamics", Nuclear Physics B, vol. 850, no. 2, pp. 242-252, Sep. 11, 2011. DOI: 10.1016/ j.nuclphysb.2011.04.018. arXiv: 1104.2503 [hep-lat].

[68] Y. Delgado Mercado and C. Gattringer, "Monte Carlo simulation of the $S U(3)$ spin model with chemical potential in a flux representation", Nuclear Physics B, vol. 862, no. 3, pp. 737750, Sep. 21, 2012. DOI: 10.1016/j .nuclphysb.2012.05.009. arXiv: 1204.6074 [hep-lat].

[69] O. Borisenko, V. Chelnokov, and S. Voloshyn, "Dual formulations of Polyakov loop lattice models", Physical Review D, vol. 102, no. 1, 014502, Jul. 6, 2020. DOI: 10.1103/physrevd. 102.014502. arXiv: 2005.11073 [hep-lat].

[70] O. Borisenko, V. Chelnokov, E. Mendicelli, and A. Papa, "Dual simulation of a Polyakov loop model at finite baryon density: Phase diagram and local observables", Nuclear Physics B, vol. 965, 115332, Apr. 2021. DOI: 10.1016/j.nuclphysb.2021.115332. arXiv: 2011.08285 [hep-lat].

[71] F. Green and F. Karsch, "Mean field analysis of $S U(N)$ deconfining transitions in the presence of dynamical quarks", Nuclear Physics B, vol. 238, no. 2, pp. 297-306, Jun. 11, 1984. DOI: $10.1016 / 0550-3213(84) 90452-8$.

[72] J. Greensite and K. Splittorff, "Mean field theory of effective spin models as a baryon fugacity expansion", Physical Review D, vol. 86, no. 7, 074501, Oct. 1, 2012. DOI: 10.1103/ physrevd.86.074501. arXiv: 1206.1159 [hep-lat].

[73] J. R. Glesaaen, M. Neuman, and O. Philipsen, "Equation of state for cold and dense heavy QCD", Journal of High Energy Physics, vol. 2016, no. 03, 100, Mar. 15, 2016. DOI: 10.1007/ JHEP03 (2016) 100. arXiv: 1512.05195 [hep-lat].

[74] J. R. Glesaaen, "Heavy quark QCD at finite temperature and density using an effective theory", PhD thesis, Johann Wolfgang Goethe-Universität Frankfurt am Main, 2016. [Online]. Available: http://nbn-resolving.de/urn:nbn:de:hebis: 30:3-440431.

[75] B. Svetitsky and L. G. Yaffe, "Critical behavior at finite-temperature confinement transitions", Nuclear Physics B, vol. 210, no. 4, pp. 423-447, Dec. 13, 1982. DOI: 10.1016/05503213(82) 90172-9.

[76] J. Polonyi and K. Szlachanyi, "Phase transition from strong-coupling expansion", Physics Letters B, vol. 110, no. 5, pp. 395-398, Apr. 8, 1982. DOI: 10.1016/0370-2693(82) 91280-1.

[77] T. Banks and A. Ukawa, "Deconfining and chiral phase transitions in quantum chromodynamics at finite temperature", Nuclear Physics B, vol. 225, no. 1, pp. 145-155, Oct. 3, 1983. DOI: $10.1016 / 0550-3213$ (83) 90016-0.

[78] J. Bartholomew, D. Hochberg, P. H. Damgaard, and M. Gross, "Effects of quarks on $S U(N)$ deconfinement phase transitions", Physics Letters B, vol. 133, no. 3-4, pp. 218-220, Dec. 15, 1983. DOI: $10.1016 / 0370-2693$ (83) 90564-6.

[79] J. Langelage, S. Lottini, and O. Philipsen, "Centre symmetric 3d effective actions for thermal $S U(N)$ Yang-Mills from strong coupling series", Journal of High Energy Physics, vol. 2011, no. 02, 57, Feb. 11, 2011. DOI: 10.1007/JHEP02 (2011) 057. arXiv: 1010.0951 [hep-lat], Erratum-ibid. Langelage, Lottini, and Philipsen [220]. 
[80] J. Langelage, G. Münster, and O. Philipsen, "Strong coupling expansion for finite temperature Yang-Mills theory in the confined phase", Journal of High Energy Physics, vol. 2008, no. 07, 36, Jul. 8, 2008. DOI: 10 .1088/1126-6708/2008/07/036. arXiv: 0805 . 1163 [hep-lat].

[81] J. Langelage, "Phase transitions in finite temperature lattice QCD from strong coupling expansions", PhD thesis, Westfälische Wilhelms-Universität Münster, 2009.

[82] M. Ogilvie, "Effective-spin model for finite-temperature QCD", Physical Review Letters, vol. 52, no. 16, pp. 1369-1372, Apr. 16, 1984. DOI: 10.1103/PhysRevLett.52.1369.

[83] A. Gocksch and M. Ogilvie, "Microcanonical determination of effective-spin models for finitetemperature QCD", Physical Review Letters, vol. 54, no. 16, pp. 1772-1774, Apr. 22, 1985. DOI: $10.1103 /$ physrevlett.54.1772.

[84] T. Heinzl, T. Kaestner, and A. Wipf, "Effective actions for the $S U(2)$ confinement-deconfinement phase transition", Physical Review D, vol. 72, no. 6, 065005, Sep. 8, 2005. DOI: 10 . 1103/physrevd.72.065005. arXiv: hep-lat/0502013.

[85] C. Wozar, T. Kaestner, A. Wipf, and T. Heinzl, "Inverse Monte Carlo determination of effective lattice models for $S U(3)$ Yang-Mills theory at finite temperature", Physical Review $D$, vol. 76 , no. 8, 085004, Oct. 4, 2007. DOI: 10.1103/physrevd.76.085004. arXiv: 0704 . 2570 [hep-lat].

[86] J. Greensite, "Potential of the effective polyakov line action from the underlying lattice gauge theory", Physical Review D, vol. 86, no. 11, p. 114 507, Dec. 17, 2012. DOI: 10.1103/ physrevd.86.114507. arXiv: 1209.5697 [hep-lat].

[87] J. Greensite and K. Langfeld, "Effective polyakov line action from strong lattice couplings to the deconfinement transition", Physical Review D, vol. 88, no. 7, 074503, Oct. 16, 2013. DOI: $10.1103 /$ physrevd.88.074503. arXiv: 1305.0048 [hep-lat].

[88] J. Greensite and K. Langfeld, "Effective polyakov line action from the relative weights method", Physical Review D, vol. 87, no. 9, 094501, May 2, 2013. DOI: 10.1103/physrevd. 87.094501. arXiv: 1301.4977 [hep-lat].

[89] J. Greensite and K. Langfeld, "Finding the effective polyakov line action for $S U(3)$ gauge theories at finite chemical potential", Physical Review D, vol. 90, no. 1, 014507, Jul. 22, 2014. DOI: 10.1103/physrevd.90.014507. arXiv: 1403.5844 [hep-lat].

[90] J. Greensite and R. Höllwieser, "Relative weights approach to $S U(3)$ gauge theories with dynamical fermions at finite density", Physical Review D, vol. 94, no. 1, 014504, Jul. 19, 2016. DOI: 10.1103/physrevd.94.014504. arXiv: 1603.09654 [hep-lat].

[91] J. Greensite and R. Höllwieser, "Finite-density transition line for QCD with $695 \mathrm{MeV}$ dynamical fermions", Physical Review D, vol. 97, no. 11, 114504, Jun. 7, 2018. DOI: 10.1103/ physrevd.97.114504. arXiv: 1708.08031 [hep-lat].

[92] G. Bergner, J. Langelage, and O. Philipsen, "Numerical corrections to the strong coupling effective Polyakov-line action for finite T Yang-Mills theory", Journal of High Energy Physics, vol. 2015, no. 11, 10, Nov. 4, 2015. DOI: 10.1007/JHEP11(2015)010. arXiv: 1505.01021 [hep-lat].

[93] O. Philipsen and J. Scheunert, "QCD in the heavy dense regime for general $N_{c}$ : On the existence of quarkyonic matter", Journal of High Energy Physics, vol. 2019, no. 11, 22, Nov. 5, 2019. DOI: 10.1007/jhep11(2019)022. arXiv: 1908.03136 [hep-lat].

[94] A. S. Christensen, J. C. Myers, and P. D. Pedersen, "Large N lattice QCD and its extended strong-weak connection to the hypersphere", Journal of High Energy Physics, vol. 2014, no. 02, 28, Feb. 6, 2014. DOI: 10.1007/JHEP02 (2014)028. arXiv: 1312.3519 [hep-lat]. 
[95] C. Gattringer and C. B. Lang, Quantum Chromodynamics on the Lattice, ser. Lecture Notes in Physics 788. Springer Berlin Heidelberg, 2010. DOI: 10.1007/978-3-642-01850-3.

[96] D. E. Knuth, "Two notes on notation", The American Mathematical Monthly, vol. 99, no. 5, pp. 403-422, May 1, 1992. DOI: 10.1080/00029890.1992.11995869. arXiv: math/ 9205211v1 [math.HO].

[97] J.-M. Drouffe and J.-B. Zuber, "Strong coupling and mean field methods in lattice gauge theories", Physics Reports, vol. 102, no. 1-2, pp. 1-119, Dec. 1983. DOI: 10.1016/03701573 (83) 90034-0.

[98] I. Montvay and G. Münster, Quantum fields on a lattice, ser. Cambridge monographs on mathematical physics. Cambridge University Press, 1994, ISBN: 0521404320.

[99] H. Meyer-Ortmanns and T. Reisz, Principles of Phase Structures in Particle Physics, ser. World Scientific Lecture Notes in Physics 77. World Scientific Publishing Co. Pte. Ltd., Dec. 2006, ISBN: 978-981-02-3441-6. DOI: 10.1142/3763.

[100] F. Green and S. Samuel, "Chiral models: Their implication for gauge theories and large N", Nuclear Physics B, vol. 190, no. 1, pp. 113-150, Jul. 6, 1981. DOI: 10.1016/05503213(81) 90486-7.

[101] M. Creutz, "On invariant integration over $S U(N)$ ", Journal of Mathematical Physics, vol. 19, no. 10, pp. 2043-2046, Oct. 1, 1978. DOI: 10.1063/1.523581.

[102] K. E. Eriksson, N. Svartholm, and B. S. Skagerstam, "On invariant group integrals in lattice QCD", Journal of Mathematical Physics, vol. 22, no. 10, pp. 2276-2278, Oct. 1, 1981. DOI: $10.1063 / 1.524760$.

[103] O. Borisenko, S. Voloshyn, and V. Chelnokov, " $S U(N)$ polynomial integrals and some applications", Reports on Mathematical Physics, vol. 85, no. 1, pp. 129-145, Feb. 2020. DOI: 10.1016/s0034-4877(20)30015-x. arXiv: 1812.06069 [hep-lat].

[104] P. M. Lo, B. Friman, and K. Redlich, "Polyakov loop fluctuations and deconfinement in the limit of heavy quarks", Physical Review D, vol. 90, no. 7, 074035, Oct. 2014. DOI: 10.1103/ physrevd.90.074035. arXiv: 1406.4050 [hep-ph].

[105] C. S. Fischer, J. Luecker, and J. M. Pawlowski, "Phase structure of QCD for heavy quarks", Physical Review D, vol. 91, no. 1, 014024, Jan. 22, 2015. DOI: 10.1103/physrevd.91.014024. arXiv: 1409.8462 [hep-ph].

[106] D. C. Brydges, "A short course on cluster expansions", in Phénomènes critiques, systèmes aléatoires, théories de jauge, Elsevier BV, 1986, pp. 129-183.

[107] G. S. Rushbrooke, G. A. Baker, and P. J. Wood, "Heisenberg model", in Phase Transitions and Critical Phenomena, Series Expansions for Lattice Models, C. Domb and M. S. Green, Eds., 1974, pp. 245-356.

[108] G. Münster, "High-temperature expansions for the free energy of vortices and the string tension in lattice gauge theories", Nuclear Physics B, vol. 180, no. 1, pp. 23-60, Jan. 26, 1981. DOI: $10.1016 / 0550-3213(81) 90153-\mathrm{x}$.

[109] H. D. Ursell, "The evaluation of gibbs' phase-integral for imperfect gases", Mathematical Proceedings of the Cambridge Philosophical Society, vol. 23, no. 6, pp. 685-697, Apr. 1927. DOI: $10.1017 / \mathrm{s} 0305004100011191$.

[110] J. E. Mayer and M. G. Mayer, Statistical Mechanics. New York, United States: John Wiley \& Sons, Inc., 1940.

[111] J. E. Mayer and E. Montroll, "Molecular distribution", The Journal of Chemical Physics, vol. 9, no. 1, pp. 2-16, Jan. 1941. DOI: 10.1063/1.1750822. 
[112] J. G. Kirkwood and Z. W. Salsburg, "The statistical mechanical theory of molecular distribution functions in liquids", Discussions of the Faraday Society, vol. 15, pp. 28-34, 1953. DOI: $10.1039 / \mathrm{df} 9531500028$.

[113] D. Ruelle, Statistical Mechanics, Rigorous Results. W. A. Benjamin, Inc., 1969.

[114] S. Poghosyan and D. Ueltschi, "Abstract cluster expansion with applications to statistical mechanical systems", Journal of Mathematical Physics, vol. 50, no. 5, 053509, May 8, 2009. DOI: 10.1063/1.3124770. arXiv: 0811.4281 [math-ph].

[115] C. Gruber and H. Kunz, "General properties of polymer systems", Communications in Mathematical Physics, vol. 22, no. 2, pp. 133-161, 1971.

[116] R. L. Dobrushin, "Estimates of semi-invariants for the Ising model at low temperatures", in Topics in Statistical and Theoretical Physics, ser. AMS Translations Series 2 177, R. L. Dobrushin, R. A. Minlos, M. A. Shubin, and A. M. Vershik, Eds., American Mathematical Society, Aug. 1996, pp. 59-81. DOI: 10.1090/trans2/177/05.

[117] A. Bovier and M. Zahradník, "A simple inductive approach to the problem of convergence of cluster expansions of polymer models", Journal of Statistical Physics, vol. 100, no. 3-4, pp. 765-778, Apr. 2000. DOI: 10.1023/a:1018631710626.

[118] G. Bergner, J. Langelage, and O. Philipsen, "Effective lattice Polyakov loop theory vs. full SU(3) yang-mills at finite temperature", Journal of High Energy Physics, vol. 2014, no. 03, 39, Mar. 6, 2014. DOI: 10.1007/JHEP03(2014)039. arXiv: 1312.7823 [hep-lat].

[119] A. Roberge and N. Weiss, "Gauge theories with imaginary chemical potential and the phases of QCD", Nuclear Physics B, vol. 275, no. 4, pp. 734-745, Dec. 29, 1986. DOI: 10.1016/05503213(86)90582-1.

[120] C. Domb, "Graph theory and embeddings", in Phase Transitions and Critical Phenomena, Series Expansions for Lattice Models, C. Domb and M. S. Green, Eds., 1974, pp. 1-95.

[121] C. Domb, "Ising model", in Phase Transitions and Critical Phenomena, Series Expansions for Lattice Models, C. Domb and M. S. Green, Eds., 1974, pp. 357-484.

[122] G. A. Baker, Quantitative Theory of Critical Phenomena. Academic Press, Inc., 1990. DoI: 10.1016/b978-0-12-075120-4.x5001-5.

[123] J. L. Martin, "Computer techniques for evaluating lattice constants", in Phase Transitions and Critical Phenomena, Series Expansions for Lattice Models, C. Domb and M. S. Green, Eds., 1974, pp. 97-112.

[124] M. Wortis, "Linked cluster expansion", in Phase Transitions and Critical Phenomena, Series Expansions for Lattice Models, C. Domb and M. S. Green, Eds., 1974, pp. 113-180.

[125] J. Oitmaa, C. Hamer, and W. Zheng, Series Expansion Methods for Strongly Interacting Lattice Models. Cambridge University Press, Apr. 2016, 338 pp., IsBn: 9780521842426.

[126] M. Lüscher and P. Weisz, "Application of the linked cluster expansion to the $n$-component $\varphi^{4}$ theory", Nuclear Physics B, vol. 300, pp. 325-359, 1988. DOI: 10.1016/0550-3213(88) 90602-5.

[127] M. Lüscher and P. Weisz, "Scaling laws and triviality bounds in the lattice $\varphi^{4}$ theory, (I). one-component model in the symmetric phase", Nuclear Physics B, vol. 290, pp. 25-60, 1987. DOI: $10.1016 / 0550-3213(87) 90177-5$.

[128] M. Lüscher and P. Weisz, "Scaling laws and triviality bounds in the lattice $\varphi^{4}$ theory, (II). one-component model in the phase with spontaneous symmetry breaking", Nuclear Physics $B$, vol. 295, no. 1, pp. 65-92, Jan. 27, 1988. DOI: 10.1016/0550-3213(88) 90228-3. 
[129] M. Lüscher and P. Weisz, "Scaling laws and triviality bounds in the lattice $\varphi^{4}$ theory, (III). n-component model", Nuclear Physics B, vol. 318, no. 3, pp. 705-741, May 29, 1989. DoI: 10.1016/0550-3213(89)90637-8.

[130] T. Reisz, "Advanced linked cluster expansion, Scalar fields at finite temperature", Nuclear Physics B, vol. 450, no. 3, pp. 569-602, Sep. 18, 1995. DOI: 10.1016/0550-3213(95)003708. arXiv: hep-lat/9505023.

[131] T. Reisz, "Hopping parameter series construction for models with non-trivial vacuum", $N u$ clear Physics B, vol. 527, no. 1-2, pp. 363-380, Aug. 31, 1998. DOI: 10.1016 / s05503213(98)00305-8. arXiv: hep-lat/9802023.

[132] H. Meyer-Ortmanns and T. Reisz, "Critical phenomena with convergent series expansions in a finite volume", Journal of Statistical Physics, vol. 87, no. 3-4, pp. 755-798, May 1997. DOI: $10.1007 /$ bf 02181244. arXiv: hep-lat/9604006.

[133] M. Campostrini, "Linked-cluster expansion of the Ising model", Journal of Statistical Physics, vol. 103, no. 1-2, pp. 369-394, Apr. 2001. DOI: 10.1023/a:1004884006193. arXiv: condmat/0005130 [cond-mat.stat-mech].

[134] J. Kim, A. Q. Pham, O. Philipsen, and J. Scheunert, "The SU(3) spin model with chemical potential by series expansion techniques", Journal of High Energy Physics, vol. 2020, no. 10, 51, Oct. 2020. DOI: 10.1007/jhep10 (2020) 051. arXiv: 2007.04187 [hep-lat].

[135] G. Horwitz and H. B. Callen, "Diagrammatic expansion for the Ising model with arbitrary spin and range of interaction", Physical Review, vol. 124, no. 6, pp. 1757-1785, Dec. 15, 1961. DOI: $10.1103 /$ physrev.124.1757.

[136] B. D. McKay and A. Piperno, "Practical graph isomorphism, II", Journal of Symbolic Computation, vol. 60, pp. 94-112, Jan. 2014. DOI: $10.1016 /$ j . jsc. 2013 .09 . 003. arXiv: 1301.1493 [cs.DM] .

[137] T. Junttila and P. Kaski, "Engineering an efficient canonical labeling tool for large and sparse graphs", in Proceedings of the Ninth Workshop on Algorithm Engineering and Experiments and the Fourth Workshop on Analytic Algorithms and Combinatorics, D. Applegate, G. S. Brodal, D. Panario, and R. Sedgewick, Eds., Society for Industrial and Applied Mathematics, Jan. 6, 2007, pp. 135-149. DOI: 10.5555/2791188.2791201.

[138] J. Riordan, "Derivatives of composite functions", Bulletin of the American Mathematical Society, vol. 52, no. 8, pp. 664-668, Aug. 1946. DOI: 10.1090/s0002-9904-1946-08621-8.

[139] C. Bloch and J. S. Langer, "Diagram renormalization, variational principles, and the infinitedimensional Ising model", Journal of Mathematical Physics, vol. 6, no. 4, pp. 554-572, Apr. 1965. DOI: $10.1063 / 1.1704307$.

[140] D. S. Gaunt and A. J. Guttmann, "Asymptotic analysis of coefficients", in Phase Transitions and Critical Phenomena, Series Expansions for Lattice Models, C. Domb and M. S. Green, Eds., 1974, pp. 181-243.

[141] A. J. Guttmann, "Asymptotic analysis of power-series expansion", in Phase Transistions and Critical Phenomena, Phase Transitions and Critical Phenomena, C. Domb and J. L. Lebowitz, Eds., 1989, pp. 3-234.

[142] M. J. George and J. J. Rehr, "Two-series approach to partial differential approximants: Three-dimensional Ising models", Physical Review Letters, vol. 53, no. 22, pp. 2063-2066, Nov. 26, 1984. DOI: 10.1103/physrevlett.53.2063.

[143] M. Hellmund and W. Janke, "Star-graph expansions for bond-diluted Potts models", Physical Review E, vol. 67, no. 2, 026118, Feb. 24, 2003. DOI: 10.1103/physreve.67.026118. arXiv: cond-mat/0206400 [cond-mat.stat-mech]. 
[144] F. Karsch, B.-J. Schaefer, M. Wagner, and J. Wambach, "Towards finite density QCD with Taylor expansions", Physics Letters B, vol. 698, no. 3, pp. 256-264, Apr. 11, 2011. DOI: 10.1016/j.physletb.2011.03.013. arXiv: 1009.5211 [hep-ph].

[145] P. de Forcrand and O. Philipsen, "Constraining the QCD phase diagram by tricritical lines at imaginary chemical potential", Physical Review Letters, vol. 105, no. 15, 152001, Oct. 4, 2010. DOI: 10.1103/physrevlett.105.152001. arXiv: 1004.3144 [hep-lat].

[146] M. Neuman, "Effective theory for heavy quark QCD at finite temperature and density with stochastic quantization", PhD thesis, Johann Wolfgang Goethe-Universität, 2015.

[147] E. T. Tomboulis, "Chiral symmetry restoration at large chemical potential in strongly coupled $S U(N)$ gauge theories", Journal of Mathematical Physics, vol. 54, no. 12, 122301, Dec. 2013. DOI: 10.1063/1.4837115. arXiv: 1304.4678 [hep-lat].

[148] E. T. Tomboulis, "Chiral symmetry in $S U\left(N_{c}\right)$ gauge theories at high density", International Journal of Modern Physics A, vol. 29, no. 25, 1445004, Oct. 3, 2014. DOI: 10.1142/ s0217751x14450043. arXiv: 1403.0664 [hep-th].

[149] J. Scheunert and O. Philipsen, "Large $N_{c}$ behaviour of lattice QCD in the heavy dense regime", in Proceedings of 37th International Symposium on Lattice Field Theory - PoS (LATTICE2019), vol. 363, Sissa Medialab, Aug. 27, 2020, 206. DOI: 10.22323/1.363.0206. arXiv: 1912.01724 [hep-lat].

[150] P. Scior and L. von Smekal, "Baryonic matter onset in two-color QCD with heavy quarks", Physical Review D, vol. 92, no. 9, 094504, Nov. 4, 2015. DOI: 10.1103/physrevd.92.094504. arXiv: 1508.00431 [hep-lat].

[151] C. Domb, "On the theory of cooperative phenomena in crystals", Advances in Physics, vol. 9, no. 35, pp. 245-361, Jul. 1960. DOI: 10.1080/00018736000101199.

[152] C. Domb and D. W. Wood, "New method of deriving high temperature expansions for the Heisenberg model", Physics Letters, vol. 8, no. 1, pp. 20-21, Jan. 1, 1964. DOI: 10.1016/ 0031-9163(64)90781-4.

[153] C. Domb and D. W. Wood, "On high temperature expansions for the Heisenberg model", Proceedings of the Physical Society, vol. 86, no. 1, pp. 1-16, Jul. 1965. DOI: 10.1088/03701328/86/1/302.

[154] M. Hellmund and W. Janke, "High-temperature series expansions for the $q$-state Potts model on a hypercubic lattice and critical properties of percolation", Physical Review E, vol. 74, no. 5, 051113, Nov. 16, 2006. DOI: 10.1103 / physreve.74.051113. arXiv: cond-mat/ 0607423 [cond-mat.stat-mech].

[155] A. Hehn, N. van Well, and M. Troyer, "High-temperature series expansion for spin-1/2 Heisenberg models", Computer Physics Communications, vol. 212, pp. 180-188, Mar. 2017. DOI: $10.1016 /$ j.cpc.2016.09.003. arXiv: 1609.01878 [cond-mat.str-el].

[156] W. J. Shugard and J. D. Weeks, "Renormalized finite-cluster method for lattice models, I. site renormalization and star cluster expansions", Physical Review B, vol. 22, no. 11, pp. 5245-5258, Dec. 1, 1980. DOI: 10.1103/physrevb.22.5245.

[157] W. Unger, "The combinatorics of lattice QCD at strong coupling", in Proceedings of The 32nd International Symposium on Lattice Field Theory - PoS (LATTICE2014), vol. 214, Sissa Medialab, May 21, 2015, 192. DOI: 10.22323/1.214.0192. arXiv: 1411.4493 [hep-lat].

[158] B. B. Brandt, G. Endrődi, and S. Schmalzbauer, "QCD phase diagram for nonzero isospinasymmetry", Physical Review D, vol. 97, no. 5, 054514, Mar. 26, 2018. DOI: 10.1103 / physrevd.97.054514. arXiv: 1712.08190 [hep-lat]. 
[159] A. W. Steiner, M. Prakash, J. M. Lattimer, and P. J. Ellis, "Isospin asymmetry in nuclei and neutron stars", Physics Reports, vol. 411, no. 6, pp. 325-375, Jun. 2005. DOI: 10.1016/ j.physrep. 2005.02.004. arXiv: nucl-th/0410066.

[160] D. T. Son and M. A. Stephanov, "QCD at a finite isospin density: From the pion to quarkantiquark condensation", Physics of Atomic Nuclei, vol. 64, no. 5, pp. 834-842, May 2001. DOI: $10.1134 / 1.1378872$. arXiv: hep-ph/0011365.

[161] M. G. Alford, A. Kapustin, and F. Wilczek, "Imaginary chemical potential and finite fermion density on the lattice", Physical Review D, vol. 59, no. 5, 054502, Jan. 29, 1999. DoI: 10. 1103/PhysRevD.59.054502. arXiv: hep-lat/9807039.

[162] J. B. Kogut and D. K. Sinclair, "Lattice QCD at finite isospin density at zero and finite temperature", Physical Review D, vol. 66, no. 3, 034505, Aug. 22, 2002. DOI: 10.1103 / PhysRevD.66.034505. arXiv: hep-lat/0202028.

[163] J. B. Kogut and D. K. Sinclair, "Quenched lattice QCD at finite isospin density and related theories", Physical Review D, vol. 66, no. 1, 014508, Jul. 31, 2002. DOI: 10.1103/PhysRevD. 66.014508. arXiv: hep-lat/0201017.

[164] B. B. Brandt, F. Cuteri, G. Endrődi, and S. Schmalzbauer, "The dirac spectrum and the BEC-BCS crossover in QCD at nonzero isospin asymmetry", Particles, vol. 3, no. 1, pp. 8086, Feb. 2020. DOI: 10.3390/particles3010007.

[165] D. T. Son and M. A. Stephanov, "QCD at finite isospin density", Physical Review Letters, vol. 86, no. 4, pp. 592-595, Jan. 22, 2001. DOI: 10.1103 / PhysRevLett . 86 . 592. arXiv: hep-ph/0005225.

[166] S. Carignano, L. Lepori, A. Mammarella, M. Mannarelli, and G. Pagliaroli, "Scrutinizing the pion condensed phase", The European Physical Journal A, vol. 53, no. 2, 35, Feb. 24, 2017. DOI: 10.1140/epja/i2017-12221-x. arXiv: 1610.06097 [hep-ph].

[167] J. B. Kogut and M. A. Stephanov, The Phases of Quantum Chromodynamics, From Confinement to Extreme Environments, ser. Cambridge Monographs on Particle Physics, Nuclear Physics and Cosmology. Cambridge University Press, Dec. 2003, vol. 21.

[168] T. D. Cohen, "Functional integrals for QCD at nonzero chemical potential and zero density", Physical Review Letters, vol. 91, no. 22, 222001, Nov. 25, 2003. DOI: 10.1103/physrevlett. 91.222001. arXiv: hep-ph/0307089.

[169] A. L. Fetter and J. D. Walecka, Quantum Theory of Many-Particle Systems. New York, United States: McGraw-Hill, 1971.

[170] J. Smit, Introduction to quantum fields on a lattice, a robust mate, Camebridge Lecture Notes in Physics vols., ser. 15. Cambridge, United Kingdom: Cambridge University Press, 2002, ISBN: 0511020783.

[171] J. Langelage and O. Philipsen, "The pressure of strong coupling lattice QCD with heavy quarks, the hadron resonance gas and the large $N$ limit", Journal of High Energy Physics, vol. 2010, no. 04, 55, Apr. 14, 2010. DOI: 10.1007/JHEP04(2010) 055. arXiv: 1002.1507 [hep-lat].

[172] V. Wiedemann, "Mean field approach to effective Polyakov loop theories", Master's thesis, Johann Wolfgang Goethe-Universität Frankfurt am Main, Dec. 2019.

[173] L. McLerran and R. D. Pisarski, "Phases of cold, dense quarks at large $N_{c}$ ", Nuclear Physics $A$, vol. 796 , no. 1 , pp. 83-100, Nov. 15, 2007. DOI: 10.1016/j.nuclphysa. 2007.08.013. arXiv: 0706.2191 [hep-ph]. 
[174] G. 't Hooft, "A planar diagram theory for strong interactions", Nuclear Physics B, vol. 72, no. 3, pp. 461-473, Apr. 18, 1974. DOI: 10.1016/0550-3213(74) 90154-0.

$[175] \quad$ B. Lucini and M. Panero, "Introductory lectures to large- $N$ QCD phenomenology and lattice results", Progress in Particle and Nuclear Physics, vol. 75, pp. 1-40, Mar. 2014. DOI: 10. 1016/j.ppnp.2014.01.001. arXiv: 1309.3638 [hep-th].

[176] M. G. Perez, "Prospects for large $n$ gauge theories on the lattice", in Proceedings of 37th International Symposium on Lattice Field Theory - PoS (LATTICE2019), vol. 363, Sissa Medialab, Aug. 27, 2020, 276. DOI: 10.22323/1.363.0276. arXiv: 2001.10859 [hep-lat].

[177] T. D. Cohen, N. Kumar, and K. K. Ndousse, "Baryons and baryonic matter in the large $N_{c}$ and heavy quark limits", Physical Review C, vol. 84, no. 1, 015204, Jul. 11, 2011. DoI: 10.1103/physrevc.84.015204. arXiv: 1102.2197 [nucl-th].

[178] P. Adhikari and T. D. Cohen, "Mean-field theory of baryonic matter for QCD in the large $N_{c}$ and heavy quark mass limits", Physical Review C, vol. 88, no. 5, 055202, Nov. 4, 2013. DOI: $10.1103 /$ physrevc.88.055202. arXiv: 1307.7725 [nucl-th].

[179] C. H. Christensen, "Exact large- $N_{c}$ solution of an effective theory for Polyakov loops at finite chemical potential", Physics Letters B, vol. 714, no. 2-5, pp. 306-308, Aug. 14, 2012. DOI: $10.1016 / \mathrm{j}$.physletb.2012.06.073. arXiv: 1204.2466 [hep-lat].

[180] O. Borisenko, V. Chelnokov, and S. Voloshyn, "The large $N$ limit of $S U(N)$ integrals in lattice models", Nuclear Physics B, vol. 960, 115177, Nov. 2020. DOI: 10.1016/j .nuclphysb. 2020.115177.

[181] G. Veneziano, "Some aspects of a unified approach to gauge, dual and gribov theories", Nuclear Physics B, vol. 117, no. 2, pp. 519-545, Dec. 27, 1976. DOI: 10.1016/0550-3213(76) 90412-0.

[182] E. Witten, "Baryons in the $1 / N$ expansion", Nuclear Physics B, vol. 160, no. 1, pp. 57-115, Nov. 26, 1979. DOI: 10.1016/0550-3213(79) 90232-3.

[183] L. McLerran, "Strongly interacting matter matter at very high energy density: 3 lectures in zakopane", in Particle physics at the dawn of the LHC, Developments in particle physics from a 50 year perspective of the Cracow school, (Zakopane, Poland), vol. 41, 2010, pp. 27992826. arXiv: 1011.3203 [hep-ph].

[184] L. McLerran, "A pedagogical discussion of quarkyonic matter and its implication for neutron stars", Acta Physica Polonica B, vol. 51, no. 5, pp. 1067-1077, 2020. DOI: $10.5506 /$ aphyspolb.51.1067.

[185] O. Philipsen and J. Scheunert, "Baryonic or quarkyonic matter?", in Proceedings of XIII Quark Confinement and the Hadron Spectrum - PoS(Confinement2018), vol. 336, Sissa Medialab, Sep. 26, 2019, 057. DOI: 10.22323/1.336.0057. arXiv: 1812.02014 [hep-lat].

[186] S. Borsányi, Z. Fodor, C. Hoelbling, S. D. Katz, S. Krieg, and K. K. Szabó, "Full result for the QCD equation of state with $2+1$ flavors", Physics Letters B, vol. 730, pp. 99-104, Mar. 7, 2014. DOI: 10.1016/j.physletb.2014.01.007. arXiv: 1309.5258 [hep-lat].

[187] HotQCD Collaboration, A. Bazavov, T. Bhattacharya, C. DeTar, H.-T. Ding, S. Gottlieb, R. Gupta, P. Hegde, U. M. Heller, F. Karsch, E. Laermann, L. Levkova, S. Mukherjee, P. Petreczky, C. Schmidt, C. Schroeder, R. A. Soltz, W. Soeldner, R. Sugar, M. Wagner, and P. Vranas, "Equation of state in (2+1)-flavor QCD", Physical Review D, vol. 90, no. 9, 094503, Nov. 4, 2014. DOI: 10.1103/physrevd.90.094503. arXiv: 1407.6387 [hep-lat].

[188] B. Lucini and M. Teper, " $S U(N)$ gauge theories in four dimensions: Exploring the approach to $N=\infty "$, Journal of High Energy Physics, vol. 2001, no. 06, 050, Jun. 23, 2001. DOI: 10.1088/1126-6708/2001/06/050. arXiv: hep-lat/0103027. 
[189] B. Lucini, M. Teper, and U. Wenger, "The high temperature phase transition in $S U(N)$ gauge theories", Journal of High Energy Physics, vol. 2004, no. 01, 061, Feb. 11, 2004. DoI: 10.1088/1126-6708/2004/01/061. arXiv: hep-lat/0307017 [hep-lat].

[190] A. Andronic, D. Blaschke, P. Braun-Munzinger, J. Cleymans, K. Fukushima, L. D. McLerran, H. Oeschler, R. D. Pisarski, K. Redlich, C. Sasaki, H. Satz, and J. Stachel, "Hadron production in ultra-relativistic nuclear collisions: Quarkyonic matter and a triple point in the phase diagram of QCD", Nuclear Physics A, vol. 837, no. 1-2, pp. 65-86, Jun. 1, 2010. DOI: $10.1016 / j$.nuclphysa.2010.02.005. arXiv: 0911.4806 [hep-ph] .

[191] L. McLerran and S. Reddy, "Quarkyonic matter and neutron stars", Physical Review Letters, vol. 122, no. 12, 122701, Mar. 26, 2019. DOI: 10.1103/physrevlett.122.122701. arXiv: 1811.12503 [nucl-th].

[192] Y. Nishida, "Phase structures of strong coupling lattice QCD with finite baryon and isospin density", Physical Review D, vol. 69, no. 9, 094501, May 5, 2004. DOI: 10.1103/PhysRevD. 69.094501. eprint: hep-ph/0312371.

[193] Y. Y. Goldschmidt, " $1 / N$ expansion in two-dimensional lattice gauge theory", Journal of Mathematical Physics, vol. 21, no. 7, pp. 1842-1850, Jul. 1980. DOI: 10.1063/1.524600.

[194] P. H. Damgaard, N. Kawamoto, and K. Shigemoto, "Chiral-symmetry restoration in lattice gauge theories at finite temperature", Physical Review Letters, vol. 53, no. 23, pp. 22112214, Dec. 3, 1984. DOI: 10.1103/physrevlett.53.2211.

[195] P. H. Damgaard, N. Kawamoto, and K. Shigemoto, "Strong coupling analysis of the chiral phase transition at finite temperature", Nuclear Physics B, vol. 264, pp. 1-28, 1986. DOI: 10.1016/0550-3213(86)90470-0.

[196] E. L. Basor and P. J. Forrester, "Formulas for the evaluation of toeplitz determinants with rational generating functions", Mathematische Nachrichten, vol. 170, no. 1, pp. 5-18, 1994. DOI: $10.1002 /$ mana. 19941700102.

[197] J. B. Conrey, D. W. Farmer, J. P. Keating, M. O. Rubinstein, and N. C. Snaith, "Autocorrelation of random matrix polynomials", Communications in Mathematical Physics, vol. 237, no. 3, pp. 365-395, Jun. 1, 2003. DOI: 10 . 1007/s00220-003-0852-2. arXiv: math-ph/0208007v2 [math-ph].

[198] J. B. Conrey, D. W. Farmer, and M. R. Zirnbauer, Howe pairs, supersymmetry, and ratios of random characteristic polynomials for the unitary groups $U(N)$, Nov. 6, 2005. arXiv: math-ph/0511024.

[199] L. Ravagli and J. J. M. Verbaarschot, "QCD in one dimension at nonzero chemical potential", Physical Review D, vol. 76, no. 5, 054506, Sep. 19, 2007. DoI: 10.1103/PhysRevD.76.054506. arXiv: 0704.1111 [hep-th].

[200] H. Weyl, The Classical Groups, Their Invariants and Representations, 2nd ed. Princeton, New Jersey: Princeton University Press, 1946, IsBN: 0-691-07923-4.

[201] R. Brower, P. Rossi, and C.-I. Tan, "The external field problem for QCD", Nuclear Physics $B$, vol. 190, no. 4, pp. 699-718, Dec. 7, 1981. DOI: 10.1016/0550-3213(81)90046-8.

[202] M. Petkovšek, H. S. Wilf, and D. Zeilberger, $A=B$. A K Peters, Ltd., 1996. [Online]. Available: http://www . math. upenn. edu/ wilf/AeqB.pdf.

[203] S. Lottini and G. Torrieri, "Percolation transition in yang-mills matter at a finite number of colors", Physical Review Letters, vol. 107, no. 15, 152301, Oct. 6, 2011. DOI: 10.1103 / physrevlett.107.152301. arXiv: 1103.4824 [nucl-th]. 
[204] S. Lottini and G. Torrieri, "Quarkyonic percolation and deconfinement at finite density and number of colors", Physical Review C, vol. 88, no. 2, 024912, Aug. 23, 2013. DOI: 10.1103/ physrevc. 88.024912. arXiv: 1204.3272 [nucl-th].

[205] D. J. Gross and E. Witten, "Possible third-order phase transition in the large- $N$ lattice gauge theory", Physical Review D, vol. 21, no. 2, pp. 446-453, Jan. 15, 1980. Dor: 10.1103/ PhysRevD.21.446.

[206] S. Necco and R. Sommer, "The $N_{f}=0$ heavy quark potential from short to intermediate distances", Nuclear Physics B, vol. 622, no. 1, pp. 328-346, Feb. 4, 2002. DOI: $10.1016 /$ S0550-3213(01)00582-X. arXiv: hep-lat/0108008.

[207] M. Fromm, J. Langelage, S. Lottini, M. Neuman, and O. Philipsen, "Onset transition to cold nuclear matter from lattice QCD with heavy quarks", Physical Review Letters, vol. 110, no. 12, 122001, Mar. 19, 2013. DOI: 10.1103/PhysRevLett.110.122001. arXiv: 1207.3005 [hep-lat].

[208] H. Arisue and T. Fujiwara, "New cluster expansion method in lattice gauge theory", Progress of Theoretical Physics, vol. 72, no. 6, pp. 1176-1196, Dec. 1984. DOI: 10.1143/PTP.72.1176.

[209] H. Arisue, T. Fujiwara, and K. Tabata, "Higher orders of the high-temperature expansion for the Ising model in three dimensions", Nuclear Physics B - Proceedings Supplements, vol. 129-130, pp. 774-776, Mar. 2004. DOI: 10 .1016/s0920-5632(03) 02709-9. arXiv: hep-lat/0309158.

[210] K. E. Iverson, A Programming Language. New York, United States: John Wiley \& Sons, Inc., 1962, ISBN: 0471430145.

[211] J. W. Essam and M. E. Fisher, "Some basic definitions in graph theory", Reviews of Modern Physics, vol. 42, no. 2, pp. 271-288, Apr. 1, 1970. DOI: 10.1103/revmodphys.42.271.

[212] S. Epp, Discrete mathematics with applications, 4th ed. Brooks/Cole Cengage Learning, 2011, ISBN: 0495391328.

[213] R. Diestel, Graph Theory, ser. Graduate Texts in Mathematics 173. Springer Berlin Heidelberg, 2017, ISBN: 978-3-662-53622-3. DOI: 10.1007/978-3-662-53622-3.

[214] I. Bars and F. Green, "Complete integration of $U(N)$ lattice gauge theory in a large- $N$ limit", Physical Review D, vol. 20, no. 12, pp. 3311-3330, Dec. 15, 1979. DOI: 10.1103 / PhysRevD.20.3311.

[215] I. Bars, "U(N) integral for the generating functional in lattice gauge theory", Journal of Mathematical Physics, vol. 21, no. 11, pp. 2678-2681, Nov. 1, 1980. DOI: 10.1063/1.524368.

[216] A. B. Balantekin, "Character expansions, itzykson-zuber integrals, and the QCD partition function", Physical Review D, vol. 62, no. 8, 085017, Sep. 26, 2000. DOI: 10.1103/physrevd. 62.085017. arXiv: hep-th/0007161.

[217] A. B. Balantekin and I. Bars, "Dimension and character formulas for Lie supergroups", Journal of Mathematical Physics, vol. 22, no. 6, pp. 1149-1162, Jun. 1, 1981. DOI: 10.1063/ 1.525038.

[218] C. Krattenthaler, "Advanced determinant calculus", in The Andrews Festschrift, Seventeen Papers on Classical Number Theory and Combinatorics, Springer Berlin Heidelberg, 2001, pp. 349-426, ISBN: 978-3-642-56513-7. DOI: 10 . $1007 / 978-3-642-56513-7$ _17. arXiv: math/9902004 [math.CO]. 
[219] C. Krattenthaler, "A determinant evaluation and some enumeration results for plane partitions", in Lecture Notes in Mathematics, Number-Theoretic Analysis, E. Hlawka and R. F. Tichy, Eds., vol. 1452, Springer Berlin Heidelberg, 1990, pp. 121-131, ISBN: 978-3-540-468646. DOI: $10.1007 / \mathrm{BFb} 0096985$.

[220] J. Langelage, S. Lottini, and O. Philipsen, "Erratum: Centre symmetric 3d effective actions for thermal $S U(N)$ Yang-Mills from strong coupling series", Journal of High Energy Physics, vol. 2011, no. 07, 14, Jul. 4, 2011. DOI: 10.1007/JHEP07 (2011) 014. 